\title{
WestVirginiaUniversity
}

THE RESEARCH REPOSITORY @ WVU

Graduate Theses, Dissertations, and Problem Reports

2011

\section{Molecular Beam Epitaxy of Multiferroic YMnO3 on c-plane GaN}

\author{
Cameron Keenan \\ West Virginia University
}

Follow this and additional works at: https://researchrepository.wvu.edu/etd

\section{Recommended Citation}

Keenan, Cameron, "Molecular Beam Epitaxy of Multiferroic YMnO3 on c-plane GaN" (2011). Graduate Theses, Dissertations, and Problem Reports. 3469.

https://researchrepository.wvu.edu/etd/3469

This Dissertation is protected by copyright and/or related rights. It has been brought to you by the The Research Repository @ WVU with permission from the rights-holder(s). You are free to use this Dissertation in any way that is permitted by the copyright and related rights legislation that applies to your use. For other uses you must obtain permission from the rights-holder(s) directly, unless additional rights are indicated by a Creative Commons license in the record and/ or on the work itself. This Dissertation has been accepted for inclusion in WVU Graduate Theses, Dissertations, and Problem Reports collection by an authorized administrator of The Research Repository @ WVU.

For more information, please contact researchrepository@mail.wvu.edu. 


\title{
Molecular Beam Epitaxy of Multiferroic $\mathbf{Y M n O}_{3}$ on c-plane GaN
}

\author{
Cameron Keenan
}

Dissertation submitted to the Eberly College of Arts and Sciences at West Virginia University

in partial fulfillment of the requirements

for the degree of

Doctor of Philosophy

in

Physics

David Lederman, Ph.D., Chair

Thomas H. Myers, Ph.D.

Larry Halliburton, Ph.D.

Sergei Urazhdin, Ph.D.

Charter Stinespring, Ph.D.

Department of Physics

Morgantown, West Virginia

2011

Keywords:

Molecular Beam Epitaxy; YMnO3; Ferroelectric Oxide

Copyright 2011 Cameron Keenan 


\section{Abstract \\ Molecular Beam Epitaxy of Multiferroic $\mathrm{YMnO}_{3}$ on c-plane GaN}

\section{Cameron Keenan}

$\mathrm{YMnO}_{3}$ is a hexagonal ferroelectric which has gained significant interest for its potential use in metal-ferroelectric-semiconductor and metal-ferroelectricmetal device structures as a means to replace currently used materials containing toxic elements, such as lead. Low coercive field and dielectric constant are just two of the advantageous properties that make this material attractive for device use, but retention and large leakage effects have become impediments to device implementation. $\mathrm{YMnO}_{3}$ is also a multiferroic material at low temperatures (below $\sim 77 \mathrm{~K}$ ) and can therefore be studied to determine the feasibility of ferroelectric-magnetic coupled control of electronic devices.

In this study, $\mathrm{YMnO}_{3}$ was deposited on metal organic chemical vapor deposition prepared $\mathrm{GaN}$ templates under a temperature range of $750{ }^{\circ} \mathrm{C}$ to $900{ }^{\circ} \mathrm{C}$ using molecular beam epitaxy. Atomic force microscopy and x-ray reflectivity revealed that the sample surfaces increased in roughness with increasing deposition temperature. X-ray diffraction showed that all the samples, except 750 ${ }^{\circ} \mathrm{C}$, were crystalline with the epitaxial relation YMO [001] $\| \mathrm{GaN}[001]$ and YMO [1-10] $\| \mathrm{GaN}$ [110]. Rocking curve analysis of the YMO (002) reflection indicated that the sample grown at $900{ }^{\circ} \mathrm{C}$ was the most crystalline, as judged by the full width half maximum of the peak.

Polarization versus voltage $(P-V)$ measurements indicated small ferroelectric response. Current versus voltage $(I-V)$ measurements displayed large leakage effects primarily due to ohmic conduction, space charge limited conduction, and Schottky emission. Estimates of the relative permittivity from the Schottky emission regime were comparable to reported values in the literature. Contamination with indium at high temperatures during growth led to the formation of highly crystalline prisms $\sim 100 \mathrm{~nm}$ in lateral size which are ferroelectric at room temperature. 


\section{Acknowledgments}

First I would like to thank my advisor Dr. David Lederman for his guidance during this project, as well as Dr. Thomas Myers for his assistance in the early stages of my research work. Both Dr. Lederman and Dr. Myers provided me with new and interesting perspectives during my time at West Virginia University and I am grateful for their instruction and teaching.

I would also like to thank my coworkers, Sandeep Chandril, Randy Tompkins, Eric Schires, Felio Perez, Kineshma Munbodh, and Phil Tabor, without whom I am sure this project would not have been completed. They were instrumental in keeping me on track to finish this work and keeping me sane during the whole process.

I am also grateful to the office staff of the West Virginia University physics department, including Sherry Puskar, Sandy Johns, Siobahn Byrne, and Devon Cleland, who provided much needed help when doing any sort of paperwork or purchasing.

I would like to thank Maria Varela at Oak Ridge National Lab for performing the TEM measurements. Without her TEM work and EELS analysis, an important discovery related to this project would not have been realized. Also, I would like to that Joe Evans from Radiant Technologies for the discussions we had concerning ferroelectric devices and associated measurements.

Lastly, I would like to thank my family and friends for being supportive during this entire venture. Without their support, I would have not been able to stay on track and not get overwhelmed. I am eternally grateful to them. 


\section{Contents}

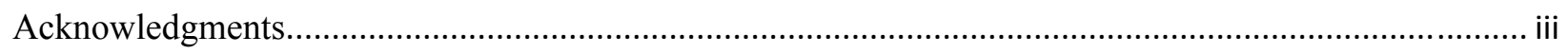

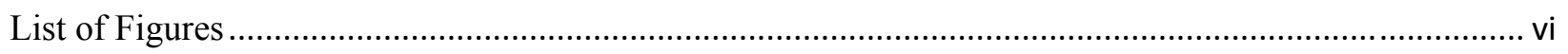

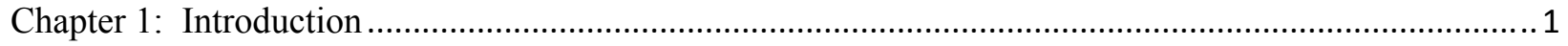

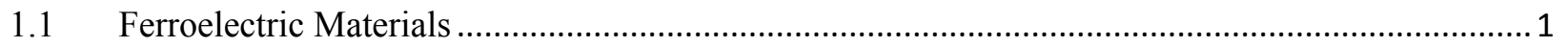

1.2 Landau-Devonshire Theory of Ferroelectric Phase Transitions .................................................. 4

1.3 Soft Phonon Mode Theory of Ferroelectricity …................................................................... 6

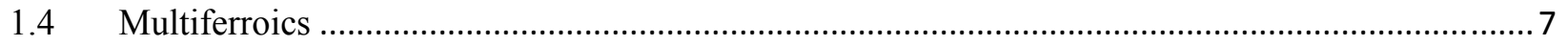

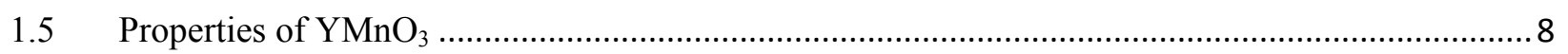

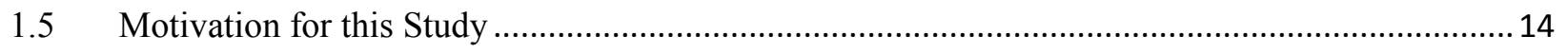

Chapter 2: Deposition and Characterization of $\mathrm{YMnO}_{3}$ Thin Films.................................................... 17

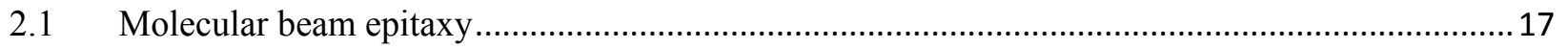

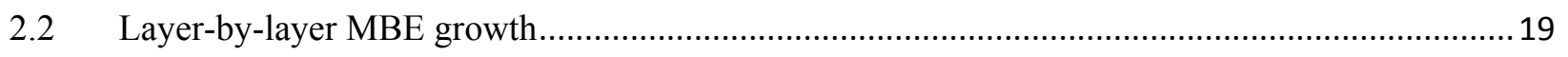

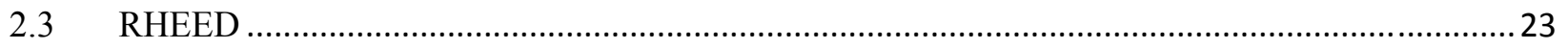

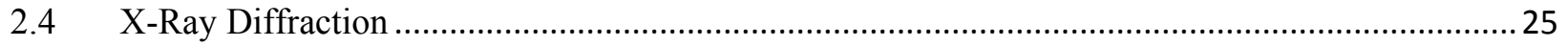

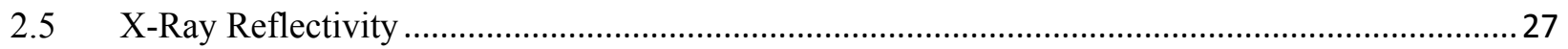

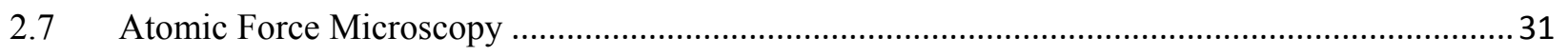

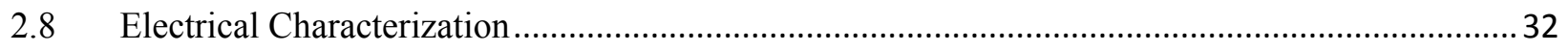

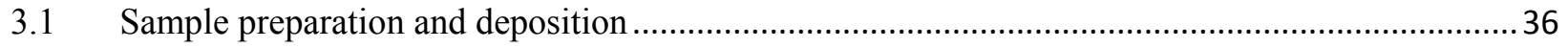

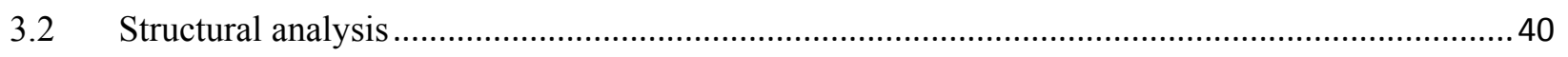

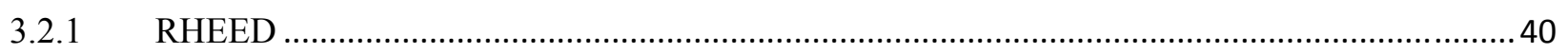

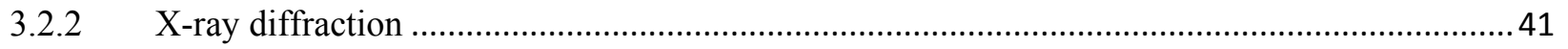

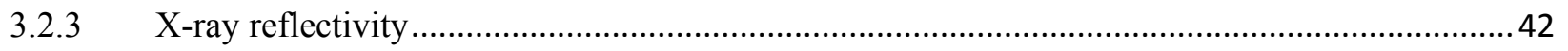

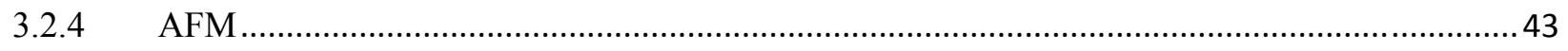

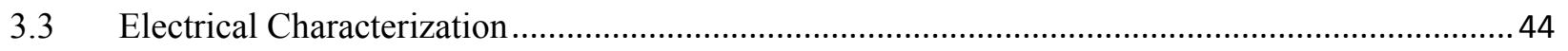

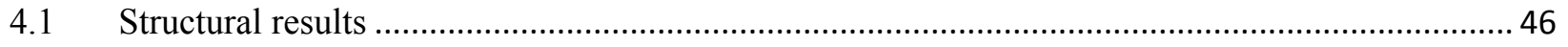

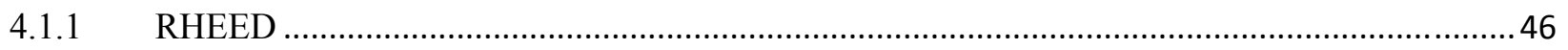

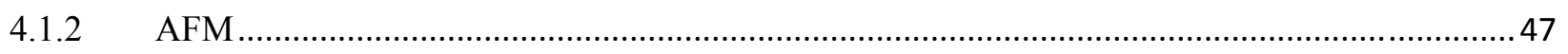

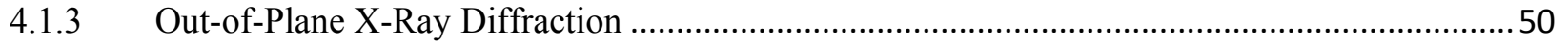

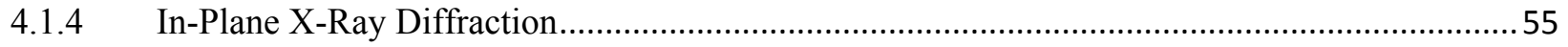

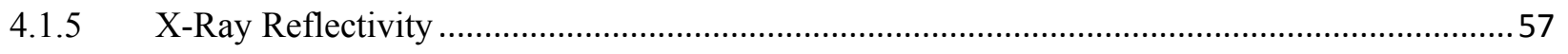




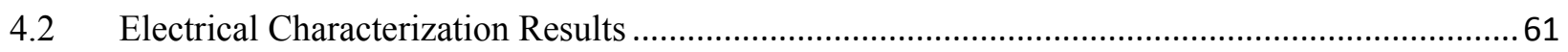

Chapter 5: Scanning Surface Potential Microscopy …................................................................... 71

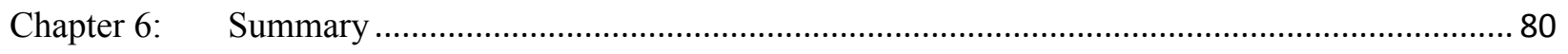

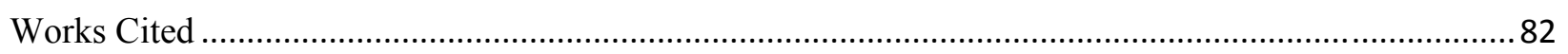

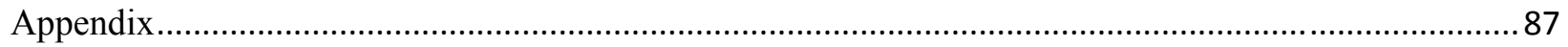

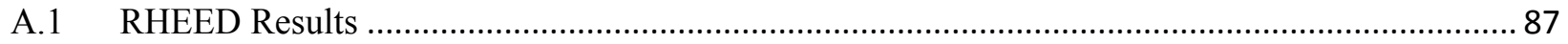

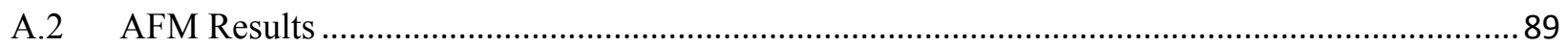

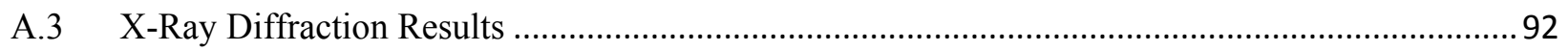

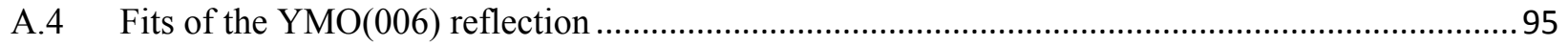

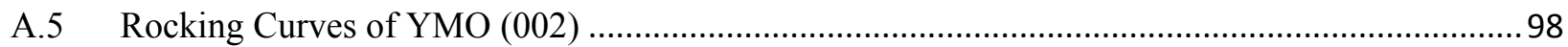

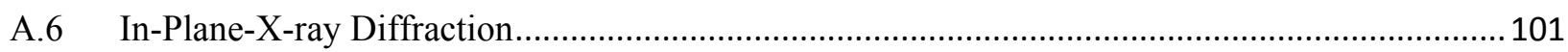

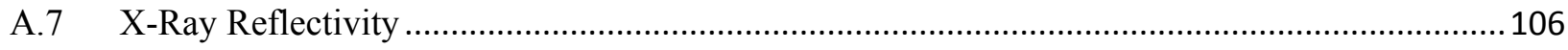

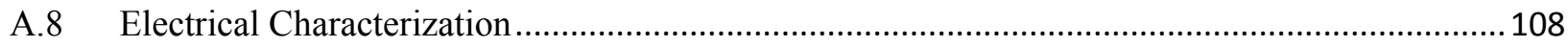

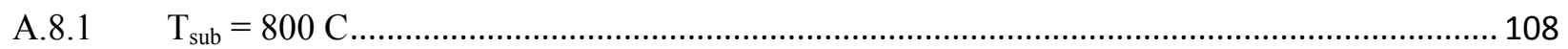

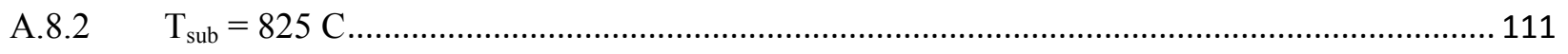

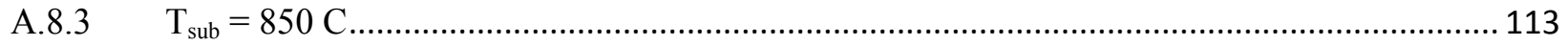

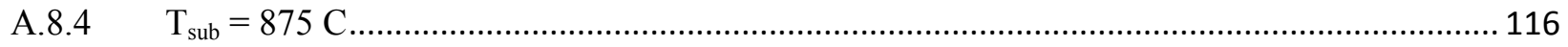

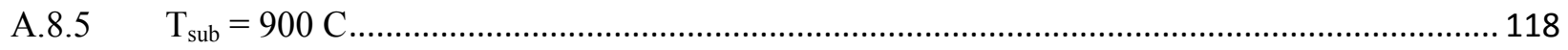




\section{List of Figures}

Figure 1: Ferroelectric hysteresis loop for a single domain single crystal (dashed line) and multi-

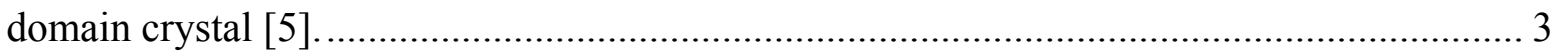

Figure 2: Polarization versus temperature for a second order phase transition (a) and for a first

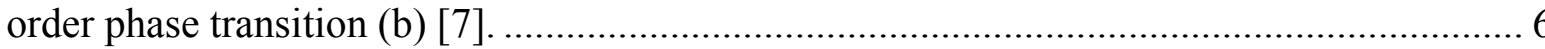

Figure 3: Crystal structure of YMO in the ferroelectric phase (space group $P 6_{3} \mathrm{~cm}$ ). Individual atoms are distinguished to account for non-equivalent lattice sites of specific species [25]. 11

Figure 4: $P-E$ curves for $\left.\mathrm{YMO} / \mathrm{Pt}(111) / \mathrm{Al}_{2} \mathrm{O}_{3}(001) .1 .4 \mathrm{a}\right)$ displays a $P_{r}$ of $0.15 \mu \mathrm{C} / \mathrm{cm}^{2}, 1.4 \mathrm{~b}$ ) displays a $P_{r}$ of $0.71 \mu \mathrm{C} / \mathrm{cm}^{2}[28]$.......

Figure 5: Epitaxial relations between $\mathrm{YMO}$ and $\mathrm{GaN}$ for the ideal (lower) and observed (upper) conditions [29].

Figure 6: Oxygen coverage based on adsorption energy for $\mathrm{GaN}(001)$ (dashed line) and

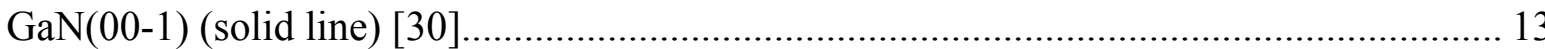

Figure 7: Diagram of an N channel MFS-FET using $\mathrm{LiNbO}_{3}$ and $\mathrm{Si}(100)$ [33].................... 15

Figure 8: Ideal band diagram of ferroelectric semiconductor interface under positive remanence (left) and negative remanence (right) [32]

Figure 9: Simplified schematic of a typical MBE chamber. Features shown common to chamber used in this work include K-cells for material evaporation, shutters to control deposition timing for each source, substrate heater and mount, and wafer transfer arm. Not displayed are pumping units, oxygen plasma source, RHEED apparatus, and quartz crystal microbalance [34]

Figure 10: Mn flux versus temperature. 
Figure 11: Mn relative flux versus temperature.

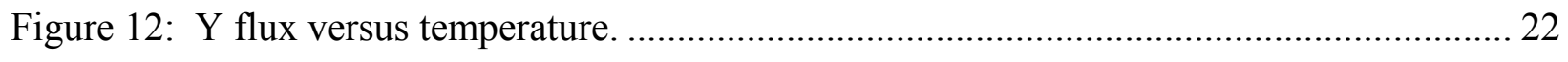

Figure 13: Y relative flux variation versus temperature. ................................................. 23

Figure 14: Simplified RHEED schematic. Incident beam angle is typically $1^{\circ}$. The fluorescent screen is located $180^{\circ}$ from the incident beam [43] ................................................ 25

Figure 15: Illustration representing x-ray diffraction from a periodic lattice. $s$ and $s 0$ are the scattered and incident $\mathrm{x}$-ray unit vectors, respectively. Hhkl is a vector perpendicular to the

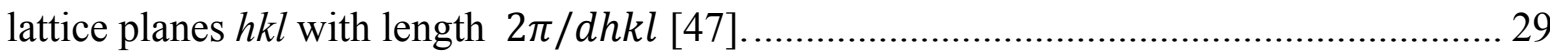

Figure 16: Two-dimensional representation of the Ewald sphere construction. The Bragg condition is satisfied for any point $h k l$ that falls on the sphere [47] ............................. 29

Figure 17: Four circle x-ray diffractometer [49] ..................................................... 30

Figure 18: Schematic of the reciprocal lattice for a [001] oriented cubic crystal. The Ewald construction is shown for a (115) reflection with $a, b$, and c corresponding to $\omega-2 \theta, \omega$, and

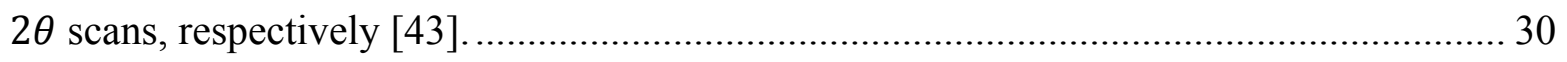

Figure 19: AFM contact (left) and tapping (right) mode operational schematics [54].............. 32

Figure 20: Sawyer-Tower circuit (a) and virtual ground method (b) testing techniques for

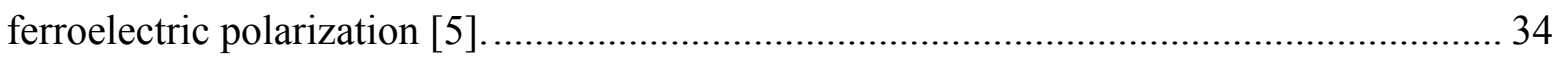

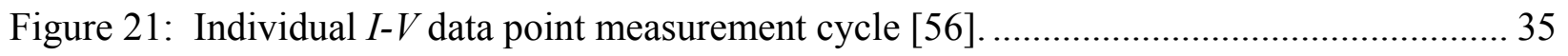

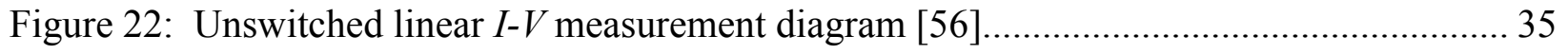

Figure 23: Shuttered RHEED oscillation captured for $850{ }^{\circ} \mathrm{C}$ sample. Arrows indicate anneal periods. The inset shows magnified region from 2750 to 3650 seconds. Each period corresponds to half a unit cell, with Y on the positive slope and Mn on the negative slope. 40 
Figure 24: Illustration of fitting model used for x-ray reflectivity. Each layer is not drawn to scale and roughness between each interface is not indicated.

Figure 25: TEM image of the interface of a sample grown under similar conditions to the samples discussed in this study.

Figure 26: RHEED for sample grown at $750^{\circ} \mathrm{C}$. (Left) GaN before deposition. (Right) Post deposition images show no clear crystalline features

Figure 27: RHEED for sample grown at $900{ }^{\circ} \mathrm{C}$. (Left) RHEED (110) lattice reflection. (Right) RHEED (1-10) lattice reflection. 47

Figure 28: $1 \mu \mathrm{m}^{2} \mathrm{AFM}$ image of sample grown at $750{ }^{\circ} \mathrm{C}$. RMS roughness was estimated to be $3.4 \AA$. 48

Figure 29: $1 \mu \mathrm{m}^{2} \mathrm{AFM}$ image of sample grown at $900^{\circ} \mathrm{C}$. RMS roughness was estimated to be $9 \AA$ 49

Figure 30: Sample roughness versus growth temperature. 49

Figure 31: X-ray diffraction of sample grown at $900{ }^{\circ} \mathrm{C}$. Arrows indicate YMO $(00 l)$ reflections. All YMO (00l) reflections are strong. Unidentifiable peaks are located to low angle side of both the (004) and (008) reflections. 51

Figure 32: Unknown peaks located next to the YMO (008) and YMO (004) reflections, left to right. 51

Figure 33: Lorentzian fit of the YMO (006) peak and nearby substrate peak. Scatter markers denote the measured data along with the associated error. Solid lines indicate the fit to each peak and the combined fit $\left(775^{\circ} \mathrm{C}\right)$ 52

Figure 34: Rocking curve of YMO (002) reflection $\left(775^{\circ} \mathrm{C}\right)$. Circles indicate measured data and the solid line a lorentzian fit. 54 
Figure 35: Comparison of the YMO (002) rocking curves for indicated growth temperatures. . 54

Figure 36: FWHM of the YMO (002) rocking curves as a function of growth temperature. ..... 55

Figure 37: Gaussian fit of the YMO (112) reflection. Scatter markers denote the measured data

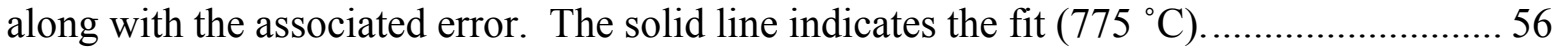

Figure 38: X-ray $\phi$ scan indicating the in-plane epitaxial relation of YMO [1-10] || GaN [110]

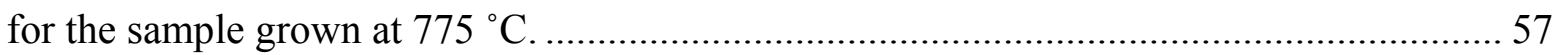

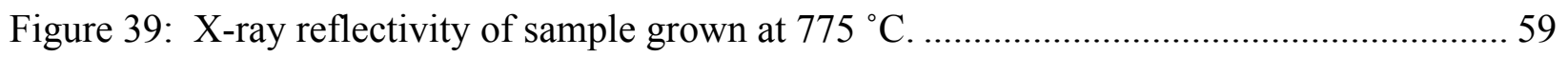

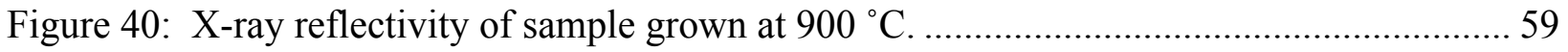

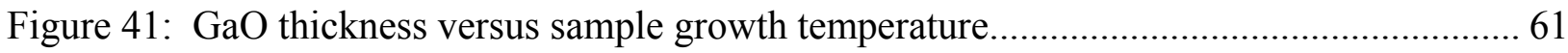

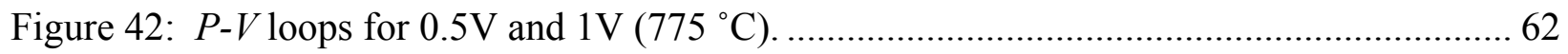

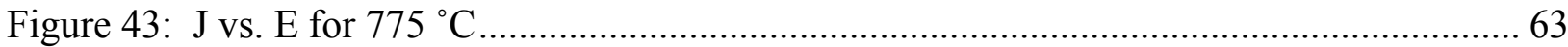

Figure 44: $\log \mathrm{J}$ vs. $\log \mathrm{E}$ for negative voltage $\left(775^{\circ} \mathrm{C}\right)$. Each solid, colored line represents a

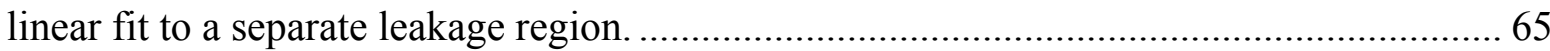

Figure 45: $\log J$ vs. $\log E$ for positive voltage $\left(775^{\circ} \mathrm{C}\right)$. Each solid, colored line represents a

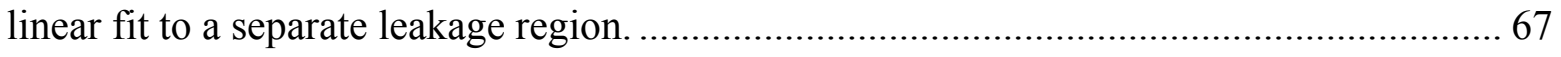

Figure 46: $\operatorname{Ln}(J / E)$ vs. $\mathrm{E}^{1 / 2}$ for positive voltage $\left(775^{\circ} \mathrm{C}\right)$. The line is the linear fit to region

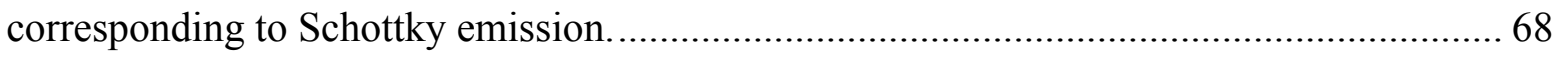

Figure 47: Various band edge alignments possible in heterostructures [45] .......................... 68

Figure 48: $\log J$ vs. $\log E$ for positive voltage $\left(900^{\circ} \mathrm{C}\right)$. Each solid, colored line represents a

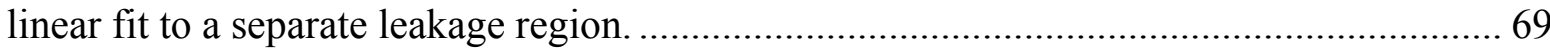

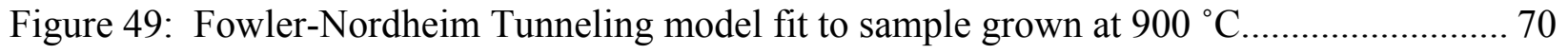


Figure 50: (a) RHEED image obtained with the electron beam along the YMO [110] direction after six unit cells of growth on GaN. (b) AFM image $\left(10 \times 10 \mu \mathrm{m}^{2}\right)$ of the same sample showing the prism structures [62].

Figure 51: High angle $\omega-2 \theta$ scan obtained for the sample in Figure 50. The green, blue, and orange curves are fits of three Gaussians to the spectrum corresponding the film, prism, and substrate $\left(\mathrm{Al}_{2} \mathrm{O} 3\right)$ peaks on a linear background (light blue line). The red line is the sum of all these contributions. The film and prism peaks correspond to the (008) reflections........ 75

Figure 52: Schematic representation of SSPM operation [61] ......................................... 75

Figure 53: The forces involved in SSPM [61] ............................................................ 75

Figure 54: (a) Average surface potential $\left(V_{S}\right)$ as a function of poling voltage $\left(V_{P}\right)$ for a $44 \mathrm{~nm}$ tall, $500 \mathrm{~nm}$ sided prism (solid circle), and for the surrounding film area (hollow circle). (b) SSPM images for the data points labeled (i)-(v) in (a). The images are $1 \times 1 \mu \mathrm{m}^{2}$ and the surface potential scale is indicated in the legend................................................... 76

Figure 55: Remanent surface potential $V_{R}$ as a function of prism surface area. The red line is a fit of the data to a straight line with an intercept of $9.5 \mathrm{mV}$ and a slope of $2.5 \times 10^{-4} \mathrm{mV} / \mathrm{nm}^{2} .77$ Figure 56: EELS scan position and spectrum for a location on one of the prism structures. The peak indicated by the "?" is located at the energy corresponding to In 78

Figure 57: TEM image of the interface between the GaN, YMO, and a prism. The materials appear to be coherent. Note that rippling in the image is due to charging effects from the $\mathrm{Al}_{2} \mathrm{O}_{3}$ 79

Figure 58: RHEED for sample grown at $775^{\circ} \mathrm{C}$. (Left) RHEED [110] lattice reflection. (Right) RHEED [100] lattice reflection. 87 
Figure 59: RHEED for sample grown at $800{ }^{\circ} \mathrm{C}$. (Left) RHEED [110] lattice reflection. (Right) RHEED [1-10] lattice reflection. 87

Figure 60: RHEED for sample grown at $825^{\circ} \mathrm{C}$. (Left) RHEED [110] lattice reflection. (Right) RHEED [1-10] lattice reflection. 88

Figure 61: RHEED for sample grown at $850{ }^{\circ} \mathrm{C}$. (Left) RHEED [110] lattice reflection. (Right) RHEED [1-10] lattice reflection. 88

Figure 62: RHEED for sample grown at $875^{\circ} \mathrm{C}$. (Left) RHEED [110] lattice reflection. (Right) RHEED [1-10] lattice reflection. 89

Figure 63: $1 \mu \mathrm{m}^{2}$ AFM image of sample grown at $775{ }^{\circ} \mathrm{C}$. RMS roughness was estimated to be $3.5 \AA$. 89

Figure 64: $1 \mu \mathrm{m}^{2}$ AFM image of sample grown at $800{ }^{\circ} \mathrm{C}$. RMS roughness was estimated to be $4.1 \AA ̊$. 90

Figure 65: $1 \mu \mathrm{m}^{2}$ AFM image of sample grown at $825^{\circ} \mathrm{C}$. RMS roughness was estimated to be $5.8 \AA \AA$. 90

Figure 66: $1 \mu \mathrm{m}^{2}$ AFM image of sample grown at $850{ }^{\circ} \mathrm{C}$. RMS roughness was estimated to be $6.4 \AA$. 91

Figure 67: $1 \mu \mathrm{m}^{2}$ AFM image of sample grown at $875^{\circ} \mathrm{C}$. RMS roughness was estimated to be $9.4 \AA$. 91

Figure 68: X-ray diffraction of YMO grown at $750{ }^{\circ} \mathrm{C}$. Arrows indicate the expected positions of the YMO $(00 l)$ reflections. 92

Figure 69: X-ray diffraction of sample grown at $775^{\circ} \mathrm{C}$. Arrows indicate the YMO $(00 l)$ reflections. All YMO $(00 l)$ reflections are strong and no other phases are observed.... 92 
Figure 70: X-ray diffraction of sample grown at $800{ }^{\circ} \mathrm{C}$. Arrows indicate the YMO $(00 l)$

reflections. All YMO (00l) reflections are strong and no other phases are observed. 93

Figure 71: X-ray diffraction of sample grown at $825^{\circ} \mathrm{C}$. Arrows indicate YMO $(00 l)$ reflections. All YMO (00l) reflections are strong. Unidentifiable peaks are located to low angle side of both the (004) and (008) reflections.

Figure 72: X-ray diffraction of sample grown at $850{ }^{\circ} \mathrm{C}$. Arrows indicate YMO $(00 l)$ reflections. All YMO (00l) reflections are strong. Unidentifiable peaks are located to low angle side of both the (004) and (008) reflections. 94

Figure 73: X-ray diffraction of sample grown at $875{ }^{\circ} \mathrm{C}$. Arrows indicate YMO $(00 l)$ reflections. All YMO (00l) reflections are strong. Unidentifiable peaks are located to low angle side of both the (004) and (008) reflections.

Figure 74: Lorentzian fit of the YMO (006) peak and nearby substrate peak. Scatter markers denote the measured data along with the associated error. Solid lines indicate the fit to each peak and the combined fit $\left(800{ }^{\circ} \mathrm{C}\right)$ 95

Figure 75: Lorentzian fit of the YMO (006) peak and nearby substrate peak. Scatter markers denote the measured data along with the associated error. Solid lines indicate the fit to each peak and the combined fit $\left(825^{\circ} \mathrm{C}\right)$ 95

Figure 76: Lorentzian fit of the YMO (006) peak and nearby substrate peak. Scatter markers denote the measured data along with the associated error. Solid lines indicate the fit to each

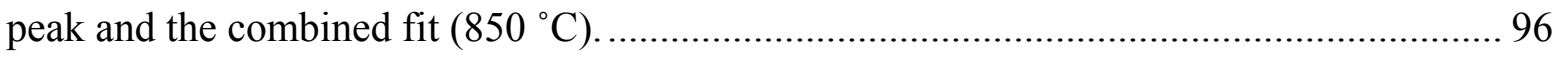

Figure 77: Lorentzian fit of the YMO (006) peak and nearby substrate peak. Scatter markers denote the measured data along with the associated error. Solid lines indicate the fit to each

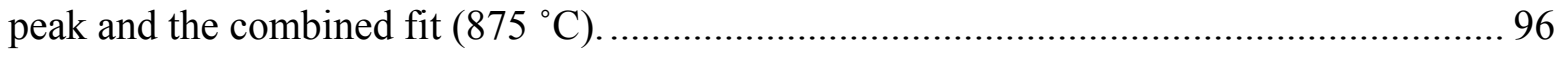


Figure 78: Lorentzian fit of the YMO (006) peak and nearby substrate peak. Scatter markers denote the measured data along with the associated error. Solid lines indicate the fit to each peak and the combined fit $\left(900{ }^{\circ} \mathrm{C}\right)$. 97

Figure 79: Rocking curve of YMO (002) reflection. Circles indicate measured data and the solid line a lorentzian fit $\left(800 \mathrm{C}^{\circ}\right)$. 98

Figure 80: Rocking curve of YMO (002) reflection. Circles indicate measured data and the solid line a lorentzian fit $\left(825^{\circ} \mathrm{C}\right)$. 98

Figure 81: Rocking curve of YMO (002) reflection. Circles indicate measured data and the solid line a lorentzian fit $\left(850^{\circ} \mathrm{C}\right)$. 99

Figure 82: Rocking curve of YMO (002) reflection. Circles indicate measured data and the solid line a lorentzian fit $\left(875^{\circ} \mathrm{C}\right)$. 99

Figure 83: Rocking curve of YMO (002) reflection. Circles indicate measured data and the solid line a lorentzian fit $\left(900{ }^{\circ} \mathrm{C}\right)$. 100

Figure 84: Gaussian fit of the YMO (112) reflection. Scatter markers denote the measured data along with the associated error. The solid line indicates the fit $\left(800{ }^{\circ} \mathrm{C}\right)$. 101

Figure 85: X-ray $\Phi$ scan indicating the in-plane epitaxial relation of YMO [1-10] || GaN [110] $\left(800 \mathrm{C}^{\circ}\right)$ 101

Figure 86: Gaussian fit of the YMO (106) reflection. Scatter markers denote the measured data along with the associated error. The solid line indicates the fit $\left(825^{\circ} \mathrm{C}\right)$ 102

Figure 87: X-ray $\Phi$ scan indicating the in-plane epitaxial relation of YMO [1-10] || GaN [110] $\left(825 \mathrm{C}^{\circ}\right)$ 102

Figure 88: Gaussian fit of the YMO (106) reflection. Scatter markers denote the measured data along with the associated error. The solid line indicates the fit $\left(850{ }^{\circ} \mathrm{C}\right)$. 103 
Figure 89: X-ray $\Phi$ scan indicating the in-plane epitaxial relation of YMO [1-10] || GaN [110] $\left(850{ }^{\circ} \mathrm{C}\right)$ 103

Figure 90: Gaussian fit of the YMO (112) reflection. Scatter markers denote the measured data along with the associated error. The solid line indicates the fit $\left(875^{\circ} \mathrm{C}\right)$ 104

Figure 91: X-ray $\Phi$ scan indicating the in-plane epitaxial relation of YMO [1-10] || GaN [110] $\left(875^{\circ} \mathrm{C}\right)$ 104

Figure 92: Gaussian fit of the YMO (112) reflection. Scatter markers denote the measured data along with the associated error. The solid line indicates the fit $\left(900{ }^{\circ} \mathrm{C}\right)$. 105

Figure 93: X-ray $\Phi$ scan indicating the in-plane epitaxial relation of YMO [1-10] || GaN [110] $\left(900{ }^{\circ} \mathrm{C}\right)$ 105

Figure 94: X-ray reflectivity of sample grown at $750{ }^{\circ} \mathrm{C}$. 106

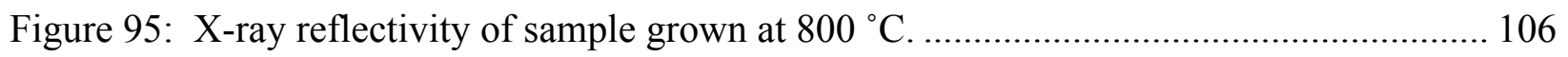

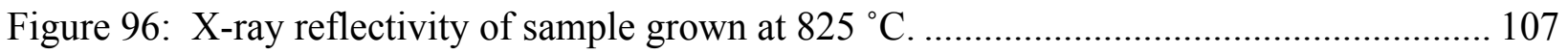

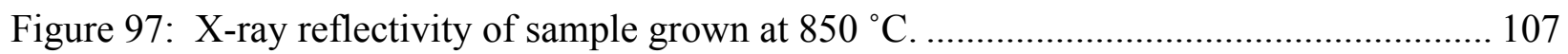

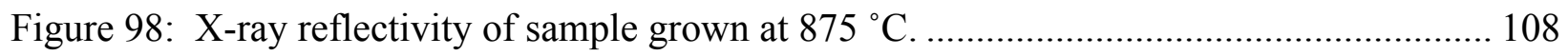

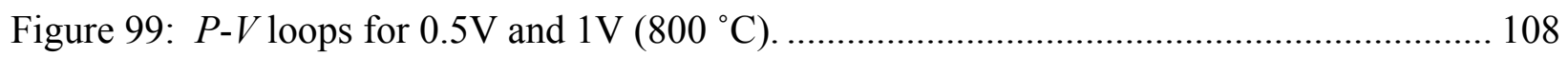

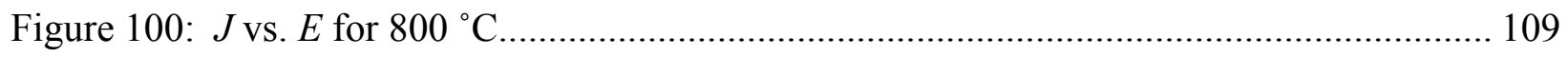

Figure 101: $\log J$ vs. $\log E$ for negative voltage $\left(800{ }^{\circ} \mathrm{C}\right)$. Each solid, colored line represents a linear fit to a separate leakage region.

Figure 102: $\log J$ vs. $\log E$ for positive voltage $\left(800{ }^{\circ} \mathrm{C}\right)$. Each solid, colored line represents a linear fit to a separate leakage region. 110

Figure 103: $\operatorname{Ln}(J / E)$ vs. $\mathrm{E}^{1 / 2}$ for positive voltage $\left(800{ }^{\circ} \mathrm{C}\right)$. The line is the linear fit to region corresponding to Schottky emission. 110 
Figure 104: $P-V$ loops for $0.5 \mathrm{~V}$ and $1 \mathrm{~V}\left(825^{\circ} \mathrm{C}\right)$.

Figure 105: $J$ vs. $E$ for $825^{\circ} \mathrm{C}$

Figure 106: $\log J$ vs. $\log E$ for negative voltage $\left(825^{\circ} \mathrm{C}\right)$. Slope is $\sim 1$ across entire range... 112

Figure 107: $\log J$ vs. $\log E$ for positive voltage $\left(825^{\circ} \mathrm{C}\right)$. Each solid, colored line represents a linear fit to a separate leakage region.

Figure 108: $\operatorname{Ln}(J / E)$ vs. $\mathrm{E}^{1 / 2}$ for positive voltage $\left(825^{\circ} \mathrm{C}\right)$. The line is the linear fit to region corresponding to Schottky emission.

Figure 109: $P-V$ loops for $0.5 \mathrm{~V}$ and $1 \mathrm{~V}\left(850{ }^{\circ} \mathrm{C}\right)$.

Figure 110: $J$ vs. $E$ for $850{ }^{\circ} \mathrm{C}$

Figure 111: $\log J$ vs. $\log E$ for negative voltage $\left(850{ }^{\circ} \mathrm{C}\right)$. Slope is $\sim 1$ across entire range... 114

Figure 112: $\log J$ vs. $\log E$ for positive voltage $\left(850^{\circ} \mathrm{C}\right)$. Each solid, colored line represents a linear fit to a separate leakage region. 115

Figure 113: $\operatorname{Ln}(J / E)$ vs. $\mathrm{E}^{1 / 2}$ for negative voltage $\left(850^{\circ} \mathrm{C}\right)$. The line is the linear fit to region corresponding to Schottky emission.

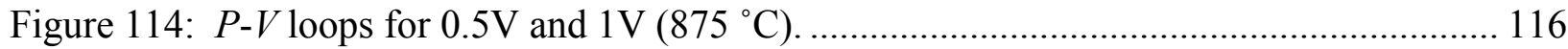

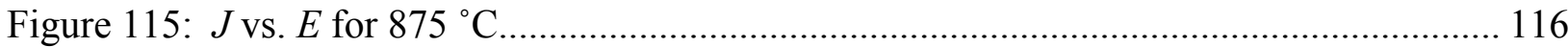

Figure 116: $\log J$ vs. $\log E$ for negative voltage $\left(875^{\circ} \mathrm{C}\right)$. Slope is $\sim 1$ across entire range... 117

Figure 117: $\log J$ vs. $\log E$ for negative voltage $\left(875^{\circ} \mathrm{C}\right)$. Each solid, colored line represents a linear fit to a separate leakage region. 117

Figure 118: $\operatorname{Ln}(J / E)$ vs. $\mathrm{E}^{1 / 2}$ for negative voltage $\left(875^{\circ} \mathrm{C}\right)$. The line is the linear fit to region corresponding to Schottky emission. 118

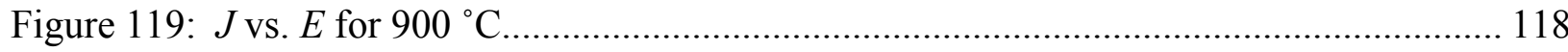

Figure 120: $\log J$ vs. $\log E$ for negative voltage $\left(900{ }^{\circ} \mathrm{C}\right)$. Slope is $\sim 1$ across entire range... 119 


\section{Chapter 1: Introduction}

\subsection{Ferroelectric Materials}

Ferroelectric materials have received a large amount of attention and research focus due to their potential for making electronic devices that lower power consumption, extend memory architecture for non-volatility and high density, and act as piezoelectric actuators [1]. The most researched materials in this field are the perovskite oxides, including lead zirconate titinate $\left(\mathrm{PbZr}_{\mathrm{x}} \mathrm{Ti}_{1-\mathrm{x}} \mathrm{O}_{3}\right.$, commonly abbreviated PZT), bismuth ferrite $\left(\mathrm{BiFeO}_{3}\right)$, and barium titinate $\left(\mathrm{BaTiO}_{3}\right)$. As lead and bismuth are both toxic and volatile and barium titinate has unresolved issues with device performance, a transition towards non-perovskite ferroelectrics is taking place, where-in a potentially important group of materials contain the rare-earths or yttrium [2].

Ferroelectrics are classified as insulating materials that display at least two stable equilibrium spontaneous polarization states that can be modified by an external field [3]. Figure 1 displays a typical polarization-voltage $(P-V)$ curve for a ferroelectric material. Properties most examined for this type of curve are the remanent polarization $\left(P_{r}\right)$, coercive field $\left(E_{c}\right)$, and spontaneous polarization $\left(P_{s}\right)$.

Crystals can be divided into 32 different point groups according to the allowed symmetry operations, i.e. reflection, rotation, etc., that return the crystal to its' original state [4]. Of the 32 different point groups, 11 have centrosymmetry. Centrosymmetry precludes spontaneous polarization, as the unit cell would display no net dipole moment [5]. Within the 21 remaining point groups, 20 
possess piezoelectricity. A piezoelectric material displays a change in magnitude of spontaneous polarization under mechanical stress. The reverse is also observed, i.e. a mechanical deformation under an applied electric field. A subset of the piezoelectric crystal groups consists of materials which display pyroelectric behavior, or a change in polarization induced by a temperature change. Lastly, 10 of the point groups are ferroelectric, having simultaneously a spontaneous and switchable polarization. 


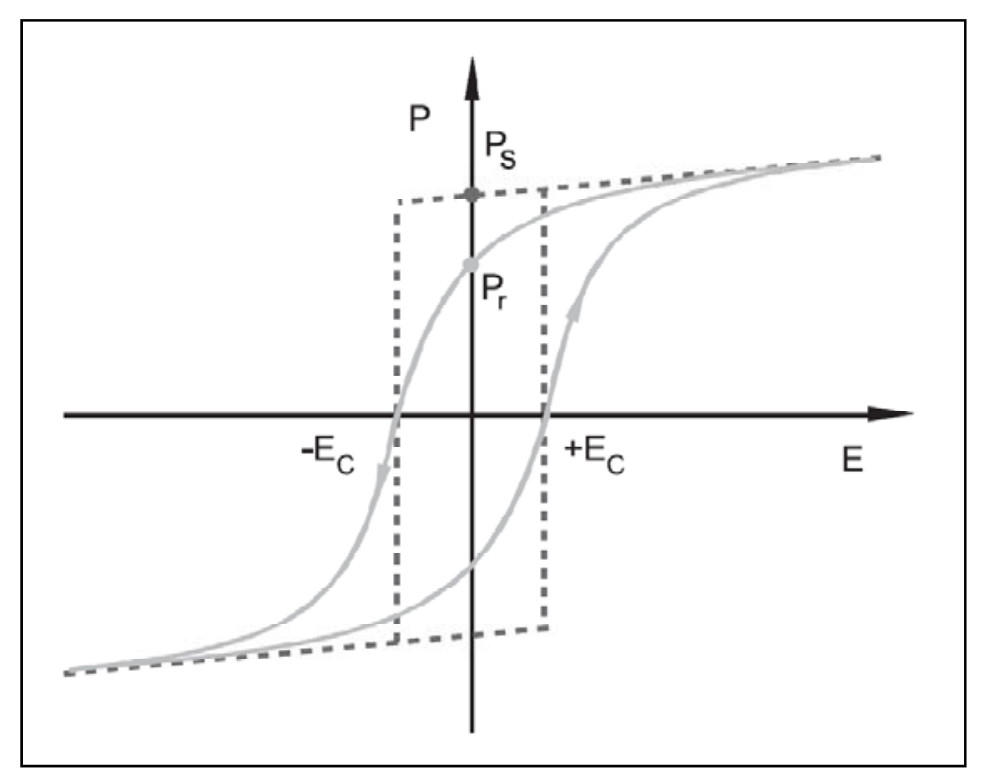

Figure 1: Ferroelectric hysteresis loop for a single domain single crystal (dashed line) and multi-domain crystal [5]. 


\subsection{Landau-Devonshire Theory of Ferroelectric Phase}

\section{Transitions}

Beginning with the Gibbs free energy [3], thermodynamic considerations can lead to expressions for the transition from a paraelectric (non-polar) phase to a ferroelectric phase. The free energy can be expanded in terms of the polarization $P$ as

$$
F(P ; T, E)=-E+g_{0}+\left(\frac{1}{2}\right) g_{2} P^{2}+\left(\frac{1}{4}\right) g_{4} P^{4}+\left(\frac{1}{6}\right) g_{6} P^{6}+\cdots
$$

where $E$ is the applied field and each of the coefficients $g_{n}$ depend on temperature [6]. For the system to be in thermal equilibrium, the energy must be at a minimum with respect to the polarization. This minimum may be found by taking the derivative of $F$ with respect to $P$ and setting it equal to zero:

$$
\frac{\partial F}{\partial P}=0=-E+g_{2} P+g_{4} P^{3}+g_{6} P^{5}+\ldots
$$

In order for a ferroelectric state to be possible, the coefficient $g_{2}$ in equation (1.1) must reach zero at some temperature $T_{0}$, leading to the expression

$$
g_{2}=\gamma\left(T-T_{0}\right)
$$

where $\gamma$ is a positive constant. It should also be noted that the second derivative of equation (1.1) gives the inverse dielectric susceptibility $\chi_{T}^{-1}$ [7]. Both $g_{2}$ and $\chi_{T}$ must become zero when $T=T_{0}$, which leads to a Curie-Weiss behavior for the paraelectric phase susceptibility 


$$
\chi_{T}=\frac{C}{T-T_{0}},
$$

where $C=\left(\partial g_{2} / \partial T\right)^{-1}$.

Using this approach, both first and second order phase transitions can be described. Second-order transitions are indicated by a smooth evolution to zero in the order parameter, in this case $P$, from one phase to another. When the coefficient $g_{4}$ is positive, higher order terms can be neglected, resulting in

$$
\gamma\left(T-T_{0}\right) P_{S}+g_{4} P_{S}^{3}=0
$$

which means that either $P_{S}=0$ or $P_{S}^{2}=\left(\gamma / g_{4}\right)\left(T_{0}-T\right)$. For the case of $T \geq$ $T_{0}$, the only possible state is $P_{S}=0$, as $\gamma$ and $g_{4}$ are both positive. For $T<T_{0}$, the polarization follows

$$
\left|P_{S}\right|=\sqrt{\left(\gamma / g_{4}\right)} \sqrt{\left(T_{0}-T\right)}
$$

Figure 2(a) is an example of a second-order phase transition [7].

First-order phase transitions can be examined under the condition that $g_{4}$ is negative. Including this stipulation requires the retention of the term $g_{6}$ as a positive, non-zero quantity in the energy expansion. Without the incorporation of this term it would be possible for the free energy to approach negative infinity, which is unphysical. This modification to the energy expansion leads to the equilibrium condition for $E=0$

$$
\gamma\left(T-T_{0}\right) P_{S}-\left|g_{4}\right| P_{S}^{3}+g_{6} P_{S}^{5}=0
$$

with possible solutions $P_{S}=0$ or

$$
\gamma\left(T-T_{0}\right)-\left|g_{4}\right| P_{S}^{2}+g_{6} P_{S}^{4}=0
$$


First order phase transitions are accompanied by a discontinuous change in the order parameter when going from $T<T_{C}$ to $T=T_{C}$, as shown in Figure 2(b).

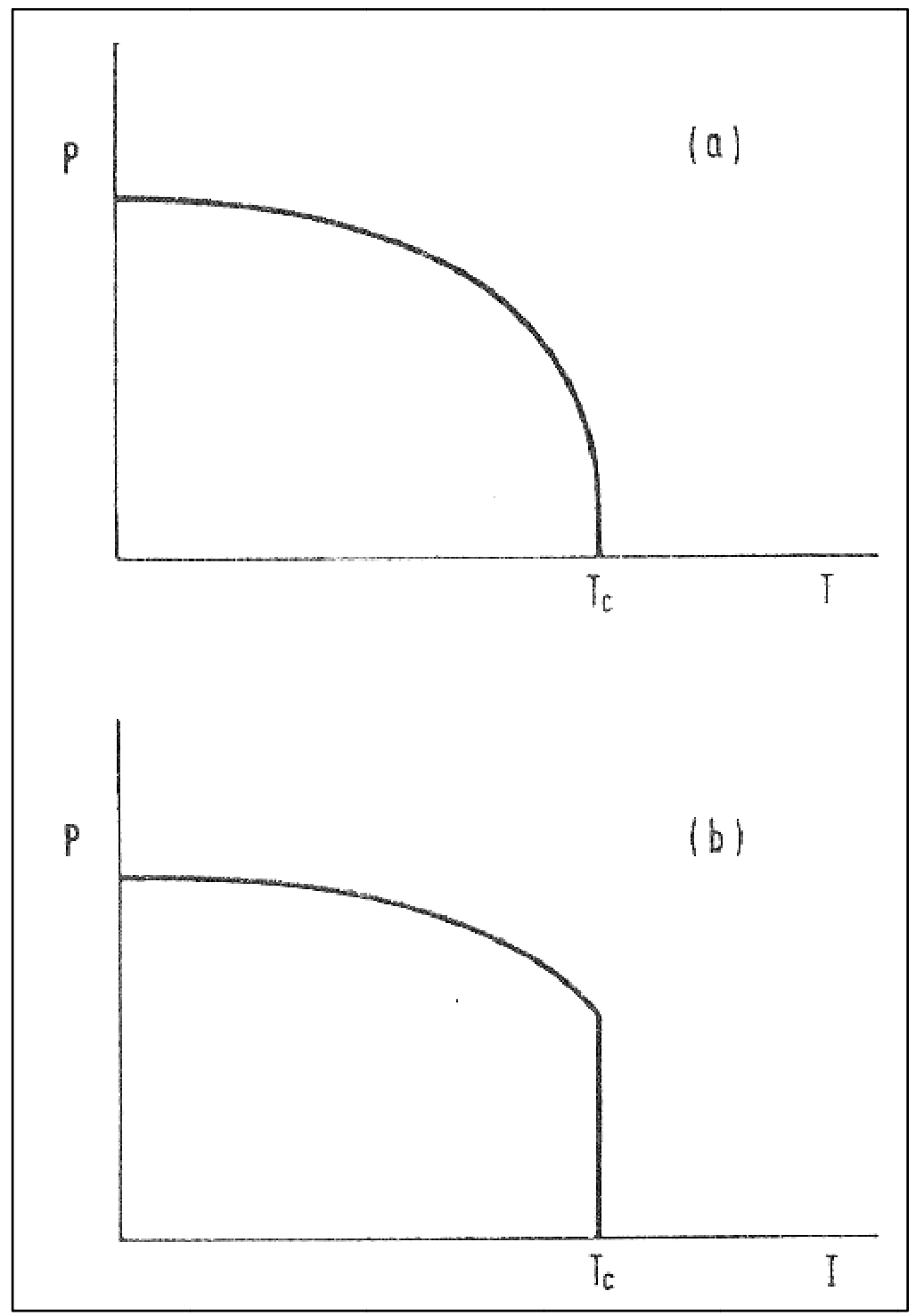

Figure 2: Polarization versus temperature for a second order phase transition (a) and for a first order phase transition (b) [7].

\subsection{Soft Phonon Mode Theory of Ferroelectricity}

Cochran [8,9] and Anderson [10] proposed in the early 1960s that ferroelectricity could result from the instability in one of the phonon modes of the 
lattice. As the temperature of the crystal is lowered through the transition temperature a "soft phonon" condenses out due to the restoring force of the lattice reaching zero. In terms of the thermodynamic quantities involved, the soft phonon interpretation of ferroelectricity can be shown to be equivalent to the Landau-Ginzburg-Devonshire theory [7].

\subsection{Multiferroics}

Multiferroics are materials in which there is more than one ferroic ordering, such as ferroelectricity and ferromagnetism [11]. Within the last few decades research interest in these materials has increased dramatically with hopes of producing devices that make use of the coupling between the two different orderings in one material. For example, the potential for creating memory devices in which the memory state can be maintained by the ferroelectric polarization and read by some means through the resulting coupling to the induced magnetization.

Multiferroics can be classified into two groups: Type-1, where the coupling is weak and the origin of each order is independent; and, Type-2, where a strong coupling allows for one ordering parameter to generate the other, for example ferroelectricity produces magnetism [11]. Hexagonal $\mathrm{YMnO}_{3}$ is a multiferroic of Type-1, where spin frustration in the anti-ferromagnetic structure allows for coupling with the net polarization. Unfortunately, $\mathrm{YMnO}_{3}$ is not antiferromagnetic until it is below roughly $77 \mathrm{~K}$, thus limiting the utility of multiferroic properties of this material. For a review of integration of multiferroic 
materials with semiconductor devices see R. Thomas et.al. [11]. For a thorough discussion of the criteria for materials to be multiferroic see N. Hill [12].

\subsection{Properties of $\mathrm{YMnO}_{3}$}

$\mathrm{YMnO}_{3}$, or $\mathrm{YMO}$, is a multiferroic compound that has gained significant interest for its ferroelectric properties and potential use for non-volatile memory applications $[2,13,14]$. It has been found to be ferroelectric below $\sim 914 \mathrm{~K}$ and antiferromagnetic below $\sim 77 \mathrm{~K}[15]$. A large number of studies have been done on this system, but issues with material quality have limited the feasibility of device implementation, particularly large leakage currents. The actual mechanism by which this material is ferroelectric is also a matter of contention $[16,17,18,19,11]$. Computational studies have suggested that YMO may be an improper ferroelectric with a significant phonon contribution to the ferroelectric transition [20,21].

Ferroelectricity in YMO was first discovered by Bertaut et.al. in 1963 [22]. Soon after this discovery, structural measurements were performed for YMO and a series of related compounds, including YbMnO3 [23], InMnO3 [24], and $\mathrm{YInO} 3$ [25], among others. Many of these compounds were found to be isostructural at room temperature, possessing the hexagonal symmetry space group $P 6_{3} \mathrm{~cm}$ illustrated in Figure 3. Yakel et.al. found that powder YMO samples had lattice parameters of $\mathrm{a}=6.125 \AA$ and $\mathrm{c}=11.41 \AA[26]$.

Thin film studies have used a variety of growth techniques including sputtering, pulsed laser deposition, rapid thermal annealing, and molecular beam 
epitaxy. Imada et. al. successfully deposited epitaxial YMO on Si (111) using $\mathrm{Y}_{2} \mathrm{O}_{3}$ buffer layers via molecular beam epitaxy with a $C-V$ memory window of $.3 \mathrm{~V}$ [27]. Ferroelectric YMO was grown via pulsed laser deposition in the metalferroelectric-metal (MFM) configuration on Pt (111)/A12O3 (001) and MgO (111) substrates by Ito et.al. [28]. Ito et.al. found that the initial stages of growth had a drastic influence on the stoichiometry and quality of the resulting material. Much like other oxide ferroelectric materials, such PZT and BFO, the amount of oxygen incorporation can affect subsequent electrical properties drastically. Ito's study displayed the production of an yttrium rich layer during the first $10 \mathrm{~nm}$ of material deposition. This study also demonstrated that through using refined deposition parameters, this non-stoichiometric layer could be removed. The use of ozone was an important factor in the stoichiometry of the resulting material. Injection of $4 \%$ ozone along with $\mathrm{O}_{2}$ allowed for samples over a range from $10^{-4}$ to $10^{-3}$ Torr to be grown with essentially identical crystal quality, as judged by the full width half maximum (FWHM) of the YMO (004) Bragg reflection. Ferroelectric measurements were improved by the incorporation of ozone into the deposition procedure along with modifications to the pulsed laser power and frequency. Figure 4 (a) and (b) show the ferroelectric $P-E$ curves taken for material produced with and without the use of ozone and optimized pulsed laser power for $200 \mathrm{~nm}$ thick films, respectively. The measured remanent polarizations, $P_{r}$, were 0.15 $\mu \mathrm{C} / \mathrm{cm}^{2}$ and $0.71 \mu \mathrm{C} / \mathrm{cm}^{2}$, respectively.

While most of studies of YMO in thin film form have focused on deposition on $\mathrm{Si}$ or metallic substrates such as $\mathrm{Pt}$, studies have been performed on other 
materials such as $\mathrm{GaN}$. Incorporation of $\mathrm{YMO}$ with $\mathrm{GaN}$ provides an interesting system to study as both materials have a hexagonal lattice, $\mathrm{GaN}$ is a wide band gap semiconductor, and $\mathrm{GaN}$ is a polar material which allows for the potential of tunable high density channel charge densities.

Several groups have produced YMO on GaN by various methods. In 2005, Posadas et.al. produced epitaxial YMO on GaN via off-axis RF magnetron sputtering with an observed remnant polarization of $2 \mu \mathrm{C} / \mathrm{cm}^{2}$, well above the value produced by Ito et.al. on $\mathrm{Pt}(111) / \mathrm{Al}_{2} \mathrm{O}_{3}(001)$ [29]. Before the successful deposition of YMO directly on GaN, the predicted lattice mismatch was $\sim 4 \%$. It was determined by Posadas' study that the epitaxial relation between the YMO and $\mathrm{GaN}$ was rotated $30^{\circ}$ in-plane with respect to the ideal orientation, causing an increased lattice mismatch of $\sim 10 \%$. This seemingly contradictory result stems from the bonding of the oxygen to the GaN surface. Calculations performed in this work suggest that the bond energy is maximized with the epitaxial relation YMO [1-10] || GaN [110] (Figure 5), as opposed to YMO [100] \| GaN [100]. First principles studies, carried out largely by Fennie and Rabe [20], suggest that Ga-O bonds at the surface cause a preferential in-plane lattice rotation. The observed rotation may also be potentially explained by the maximum amount of oxygen that can bond to the GaN surface. Zywietz et. al. concluded that oxygen coverage of $\mathrm{GaN}(001)$ reaches a saturation point of roughly $80 \%$ of a ML [30]. Incorporating this restriction of oxygen coverage can lead to a preferential rotation of $\mathrm{YMO}$ by $30^{\circ}$, giving a lattice alignment of 9 unit cells of $\mathrm{GaN}$ to 4 unit 
cells of YMO. With this configuration, the resulting lattice mismatch is roughly $1 \%$.

Molecular beam epitaxy has also been used to fabricate $\mathrm{YMO} / \mathrm{GaN}$ heterostructures in an attempt to produce metal-ferroelectric-semiconductor (MFS)-FETs; or, in the case of the use of an interstitial insulating layer, metalferroelectric-insulator-semiconductor (MFIS)-FETs. Studies, such as Chye et al. [31], have also demonstrated the same basal plane rotation that has accompanied other deposition methods and found a remanent polarization of $3.2 \mu \mathrm{C} / \mathrm{cm}^{2}$. While these values are sufficient for MFS-FET device operation, leakage effects and retention have been issues for device construction.

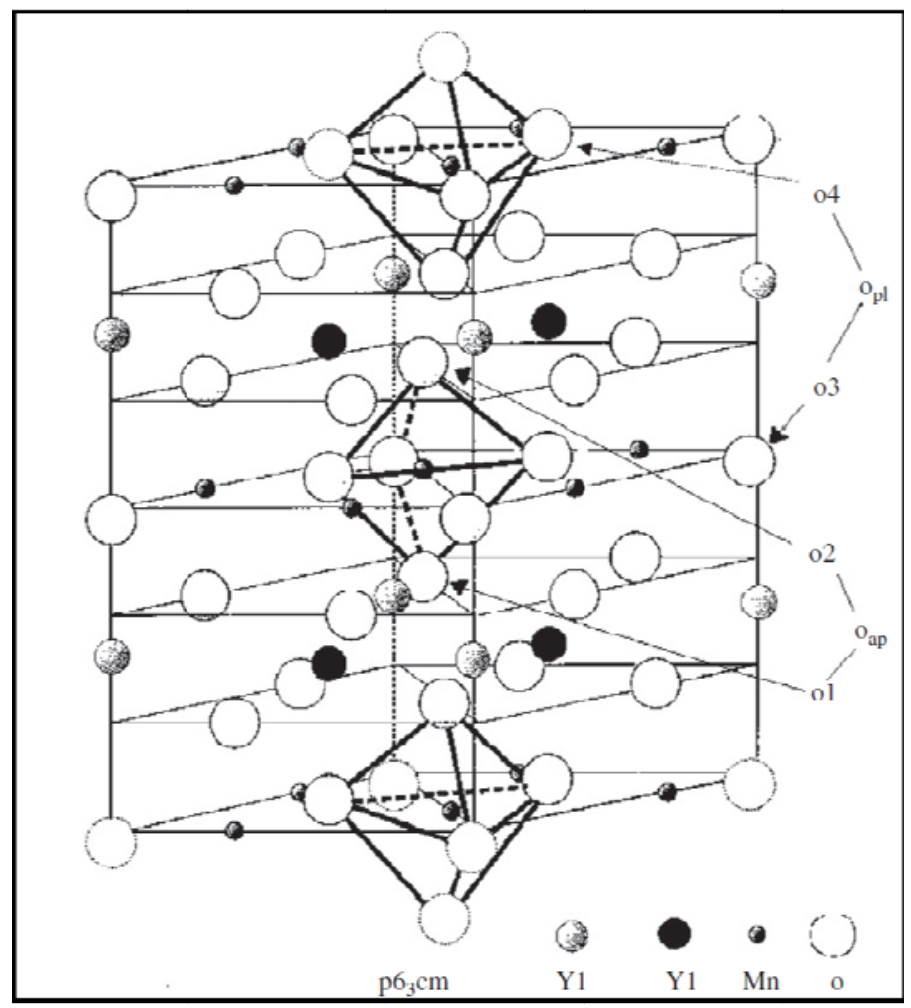

Figure 3: Crystal structure of YMO in the ferroelectric phase (space group $P 6_{3} \mathrm{~cm}$ ). Individual atoms are distinguished to account for non-equivalent lattice sites of specific species [25]. 


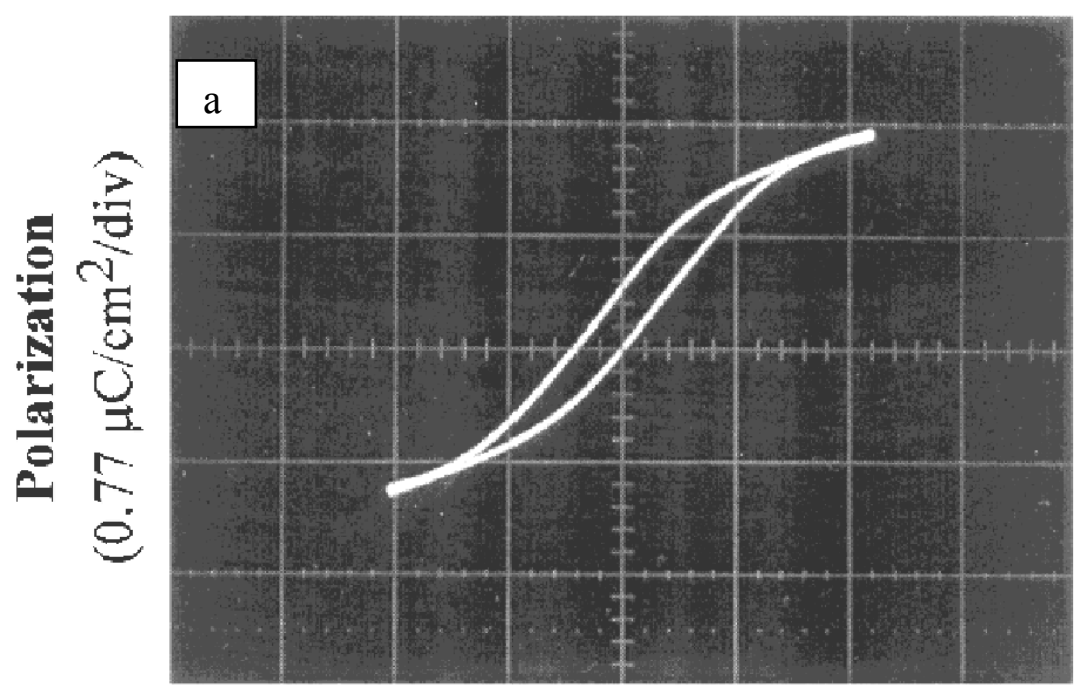

Electric Field

$(122 \mathrm{kV} / \mathrm{cm} / \mathrm{div})$

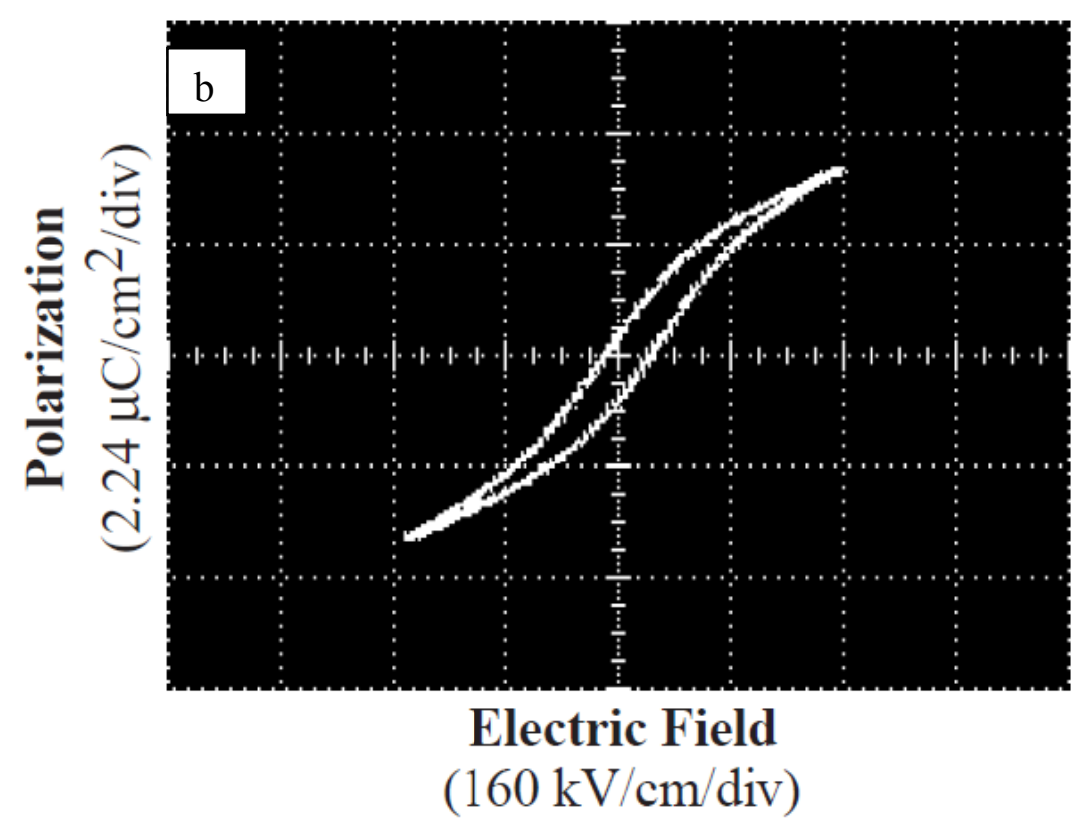

Figure 4: $P$ - $E$ curves for $\mathrm{YMO} / \mathrm{Pt}(111) / \mathrm{Al}_{2} \mathrm{O}_{3}(001)$. 1.4a) displays a $P_{r}$ of 0.15 $\mu \mathrm{C} / \mathrm{cm}^{2}, 1.4 \mathrm{~b}$ ) displays a $P_{r}$ of $0.71 \mu \mathrm{C} / \mathrm{cm}^{2}$ [28]. 


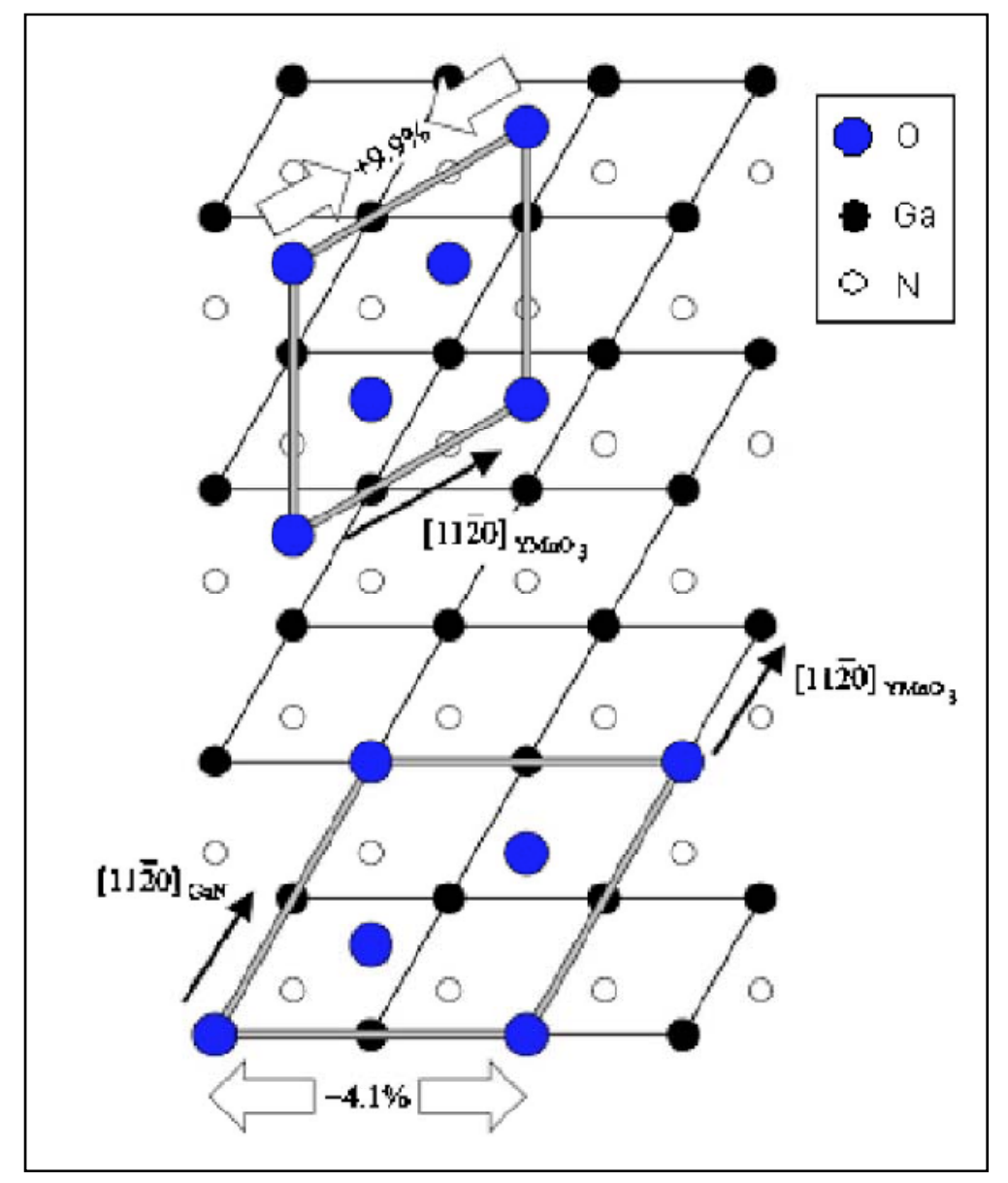

Figure 5: Epitaxial relations between $\mathrm{YMO}$ and $\mathrm{GaN}$ for the ideal (lower) and observed (upper) conditions [29].

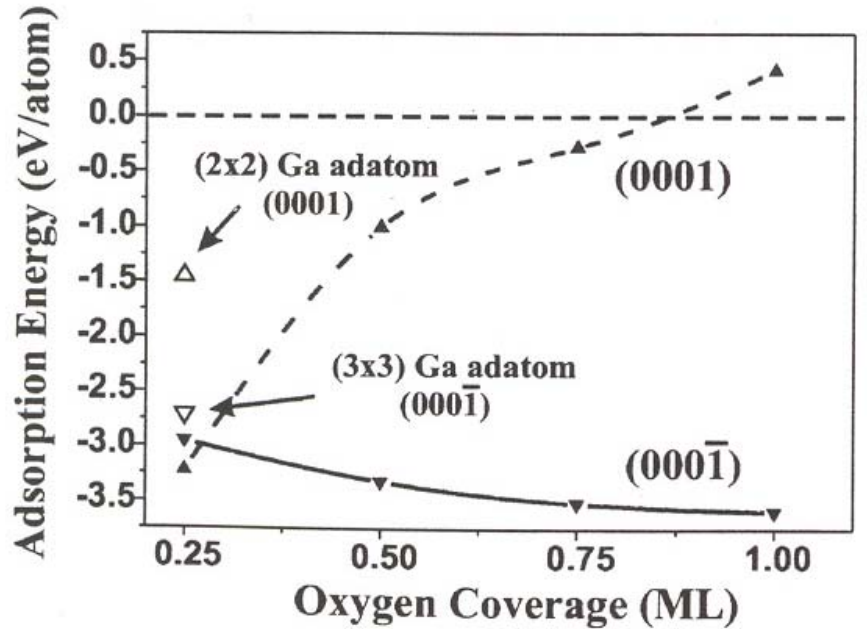

Figure 6: Oxygen coverage based on adsorption energy for $\mathrm{GaN}(001)$ (dashed line) and $\mathrm{GaN}(00-1)$ (solid line) [30]. 


\subsection{Motivation for this Study}

MFS-FETs have gained interest for their potential applications for nonvolatile memory applications with non-destructive read operations. S. H. Wu proposed the use of the ferroelectric field effect as a means to construct transistors in 1974 [32]. Since then a large amount of research has been focused on finding suitable combinations of metals, ferroelectrics, and semiconductors that will have memory retention and switching characteristics appropriate for modern device structures.

MFS-FETs utilize accumulation and inversion of a semiconductor to provide a means by which to read a memory state provided by the polarization direction in a ferroelectric layer. Figure 7 shows an example of a MFS-FET using $\mathrm{LiNbO}_{3}$ on $\mathrm{Si}$ (100) [33]. The ferroelectric in this example, $\mathrm{LiNbO}_{3}$, can have its polarization reversed, or switched, via the application of a voltage between the gate and source electrodes. Once the ferroelectric is polarized, corresponding to a 1 or 0 memory state, the electric field can be removed and the memory state will remain. Subsequently, reading the current between the source and drain for a suitable applied voltage can result in a non-destructive readout of the memory state of the ferroelectric. Figure 8 shows the band diagram for an ideal MFS structure [32]. For the case of an $\mathrm{N}$ type semiconductor, the left and right sides of Figure 8 correspond to accumulation and inversion, respectively. In the case of a $\mathrm{P}$ type semiconductor, the conditions for inversion and accumulation are reversed. When either channel device is used, the conduction through the channel between the source and drain can be controlled by the polarization state of the ferroelectric. 
While MFS-FETs have the potential to reduce power consumption, resulting from non-volatility, and increase the speed of device operation, as read operations would be non-destructive, actual implementation of these devices has suffered mostly due to poor ferroelectric retention and large leakage effects.

The aim of this study is to examine the MFS capacitor structure using YMO and $\mathrm{GaN}$ as the ferroelectric and semiconductor, respectively. Research up to this point on YMO as a material for MFS-FETs has focused largely on structures using Si. This study aims to extend the knowledge about the conduction properties of this material by looking at leakage mechanisms for MFS capacitors grown by molecular beam epitaxy.

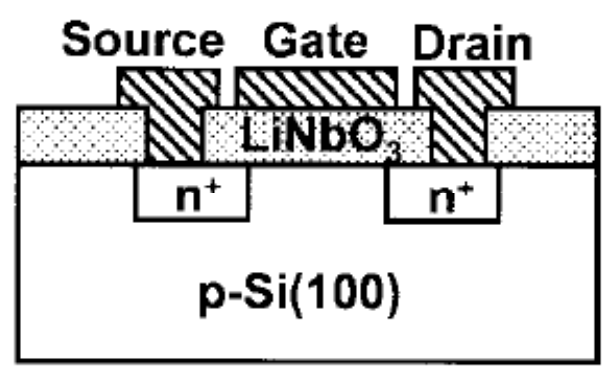

Figure 7: Diagram of an $\mathrm{N}$ channel MFS-FET using $\mathrm{LiNbO}_{3}$ and $\mathrm{Si}$ (100) [33]. 


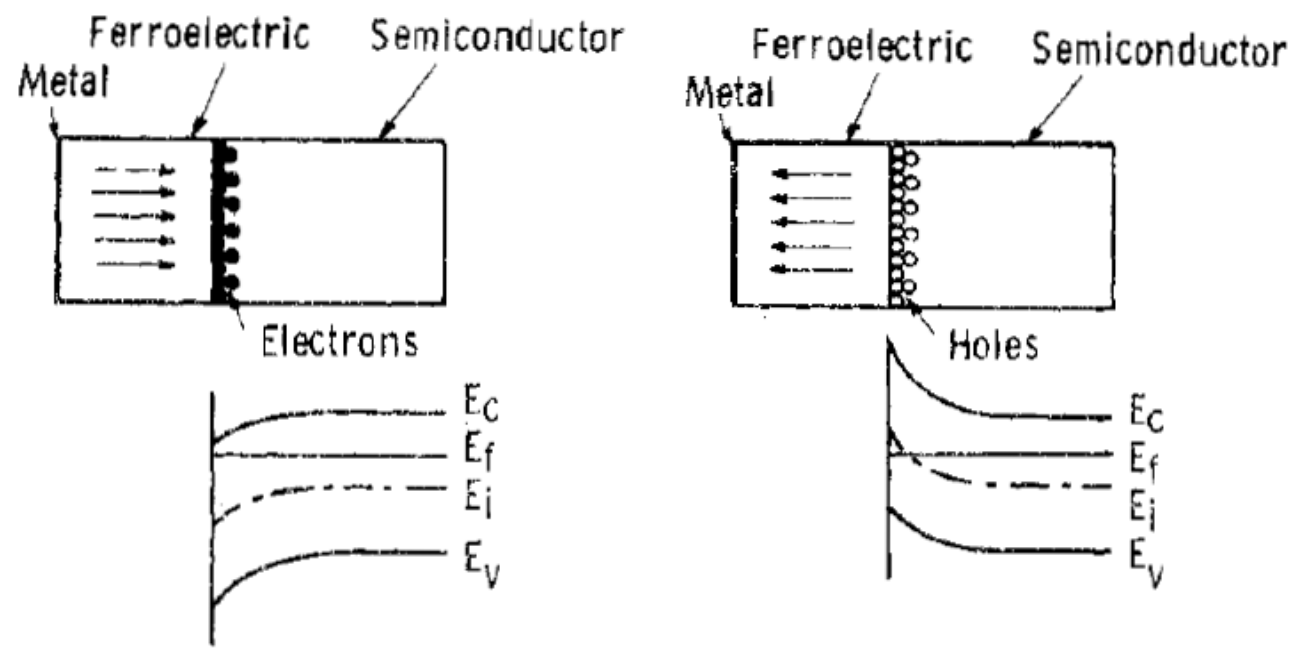

Figure 8: Ideal band diagram of ferroelectric semiconductor interface under positive remanence (left) and negative remanence (right) [32]. 


\section{Chapter 2: Deposition and Characterization of $\mathrm{YMnO}_{3}$ Thin Films}

Molecular beam epitaxy (MBE) was used to deposit $\mathrm{YMnO}_{3}$ (YMO) thin films on GaN (001) templates prepared via metal organic chemical vapor deposition (MOCVD). Reflection high energy electron diffraction (RHEED) was monitored during deposition to evaluate surface quality. Crystal quality, sample thickness, and interfacial roughness were determined using x-ray diffraction and x-ray reflectivity (XRR). Surface morphology was determined using atomic force microscopy (AFM). Electrical characterization was performed on capacitor structures of $400 \mu \mathrm{m}^{2}$ platinum top contacts and $\mathrm{GaN}$ bottom contacts to determine the polarization and leakage properties of the films.

\subsection{Molecular beam epitaxy}

Figure 9 displays a simple schematic of a typical MBE system [34]. As the technique is aptly named, beams of elemental or compound materials are deposited directly onto a substrate where chemical and/or structural reconstitution takes place. "Epitaxy" refers to the process by which the adsorbates arrange themselves according to the substrate surface. In the fields of semiconductor and materials research, MBE (typical background pressure of $10^{-10}$ Torr) has provided a means of sample preparation with lower contamination than other growth 
methods, such as vapor phase epitaxy or sputtering. MBE also affords the use of various in-situ techniques for sample characterization that are unavailable using methods that do not provide comparably low background pressures. Some examples of the analysis methods that can be implemented are RHEED [35], Auger Electron Spectroscopy (AES) [36], ellipsometry [37], and laser interferometric methods [38].

Along with allowing for low pressure in-situ characterization techniques, MBE also gives the grower the ability to create abrupt, atomic interfaces. With the level of control provided by MBE, homoepitaxy [39], heterojunctions [40,41], and quantum well structures [42] have been fabricated with high quality, sharp interfaces.

Several of the features that set MBE apart from other techniques have been mentioned briefly, but deserve a more thorough treatment. Probably the most important aspect of MBE is the pressures at which material growth is performed. Typical MBE chambers have a source to substrate distance of approximately 0.2 m. The mean free path of a molecule or atom can be expressed as

$$
L_{b}^{-1}=\sqrt{2} \pi n_{b} d_{b}^{2}+\pi n_{g} d_{b g}^{2} \sqrt{1+v_{g}^{2} / v_{b}^{2}}
$$

where $n_{b}, d_{b}$, and $v_{b}$ are the concentration, diameter, and average velocity of the molecules of the deposition beam, respectively [43]. Terms with a subscript $g$ denote the corresponding parameters for the residual gas in the chamber and $d_{b g}=\left(d_{b}+d_{g}\right) / 2$. Even in pressures approaching $10^{-6}$ Torr, the mean free path of most materials is of the order of $10 \mathrm{~m}$. This provides the unique ability for 
the material constituents to react only at the sample surface, thus providing stringent stoichiometry control.

One of the most notable methods used for material evaporation during MBE consists of Knudsen cells (k-cell). K-cells operate under a purely thermal process where one or a series of resistive filaments are wound around a chemically nonreactive crucible containing a deposition material. Using an appropriate thermocouple placed in contact with the crucible and temperature controllers with proper feedback implementation, source temperatures can be stabilized to within $\pm .1{ }^{\circ} \mathrm{C}$. Deposition is typically done at a growth rate of roughly 1 monolayer/s, which provides adequate surface migration such that a smooth surface is ensured [43].

\subsection{Layer-by-layer MBE growth}

Many material growth procedures using MBE involve the simultaneous deposition of several sources and chemical or physical means, such as desorption, control the stoichiometry of the resulting material. Several examples of this include the deposition of most of the III-V semiconductor family, where one of the constituents limits the growth process.

An alternate growth method involves a purely physical practice of composition control where-in the shutters are alternately opened and closed to provide the desired material structure. This method has been successfully implemented for complex crystalline materials where self assembly would not be possible, such as in the case of cuprate superconductors [44]. While using layer- 
by-layer deposition provides more direct control of the intended composition, mechanisms such as interdiffusion, desorption, and pre-deposition flux (atoms $/ \mathrm{cm}^{2} \mathrm{~s}$ ) calibration tend to have an increased influence on the final product. Precise temperature control of the sources becomes particularly important. Flux from a thermally emitting source under MBE conditions follows [45]

$$
F=\frac{P}{\sqrt{2 \pi m k_{B} T}} \sim \frac{3.5 \times 10^{22} P(\text { Torr })}{\sqrt{m(\mathrm{~g} / \mathrm{mol}) T(\mathrm{~K})}} \text { atoms } / \mathrm{cm}^{2} \mathrm{~s} .
$$

Using equation (2.2), the surface site density of each element, and the relative flux variation for $\mathrm{a}+/-0.1^{\circ}$ temperature stability, the stoichiometry deviation can be estimated. Following standard error analysis,

$$
\delta x=\sqrt{.04 \times\left(\delta_{M n}^{2}+\delta_{Y}^{2}\right)},
$$

where $\delta_{M n}$ and $\delta_{Y}$ are the relative flux variations. For the temperatures noted in Table 1 , the expected variation in stoichiometry should be roughly $1 \%$. Figure 10 through Figure 13 display the fluxes and relative flux variations calculated from vapor pressure curves supplied by Veeco. 


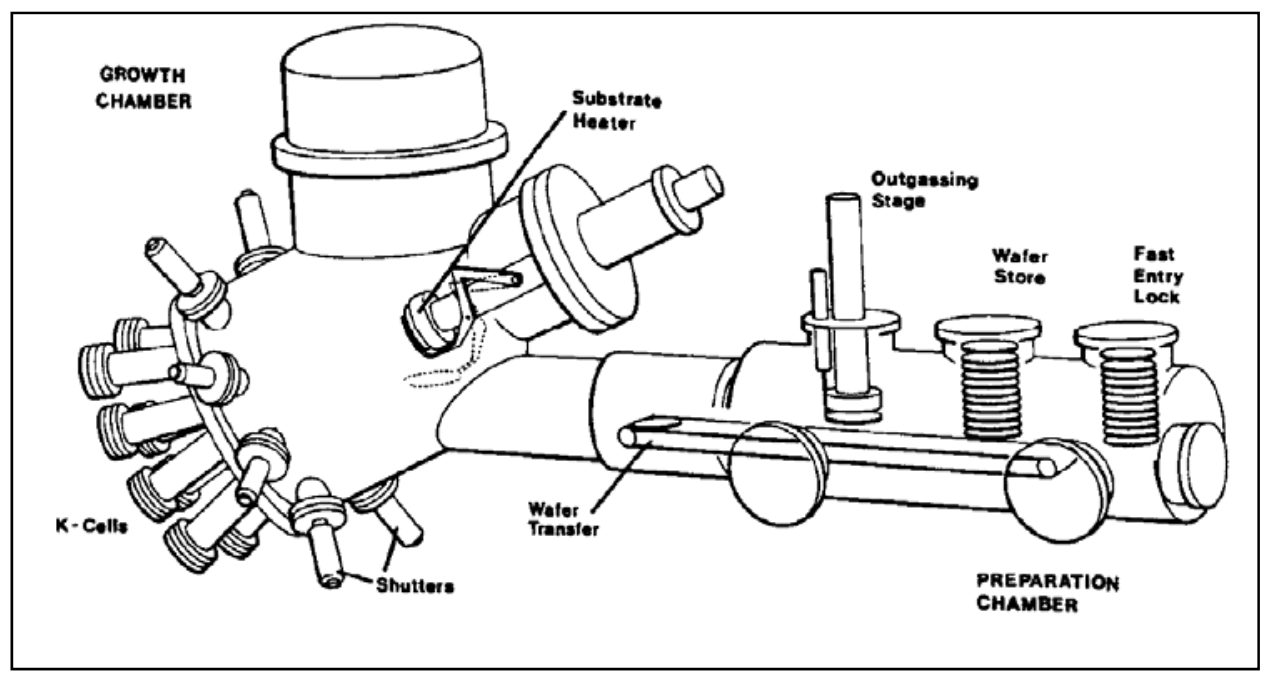

Figure 9: Simplified schematic of a typical MBE chamber. Features shown common to chamber used in this work include K-cells for material evaporation, shutters to control deposition timing for each source, substrate heater and mount, and wafer transfer arm. Not displayed are pumping units, oxygen plasma source, RHEED apparatus, and quartz crystal microbalance [34].

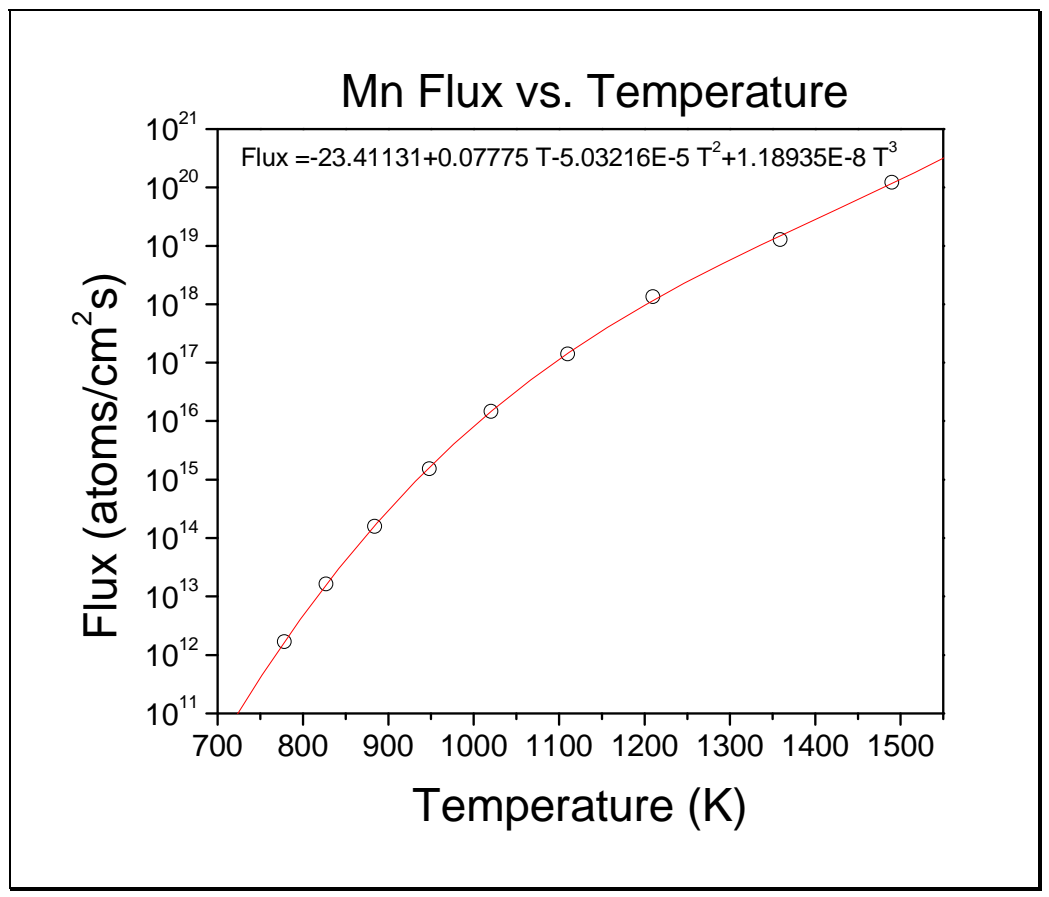

Figure 10: Mn flux versus temperature. 


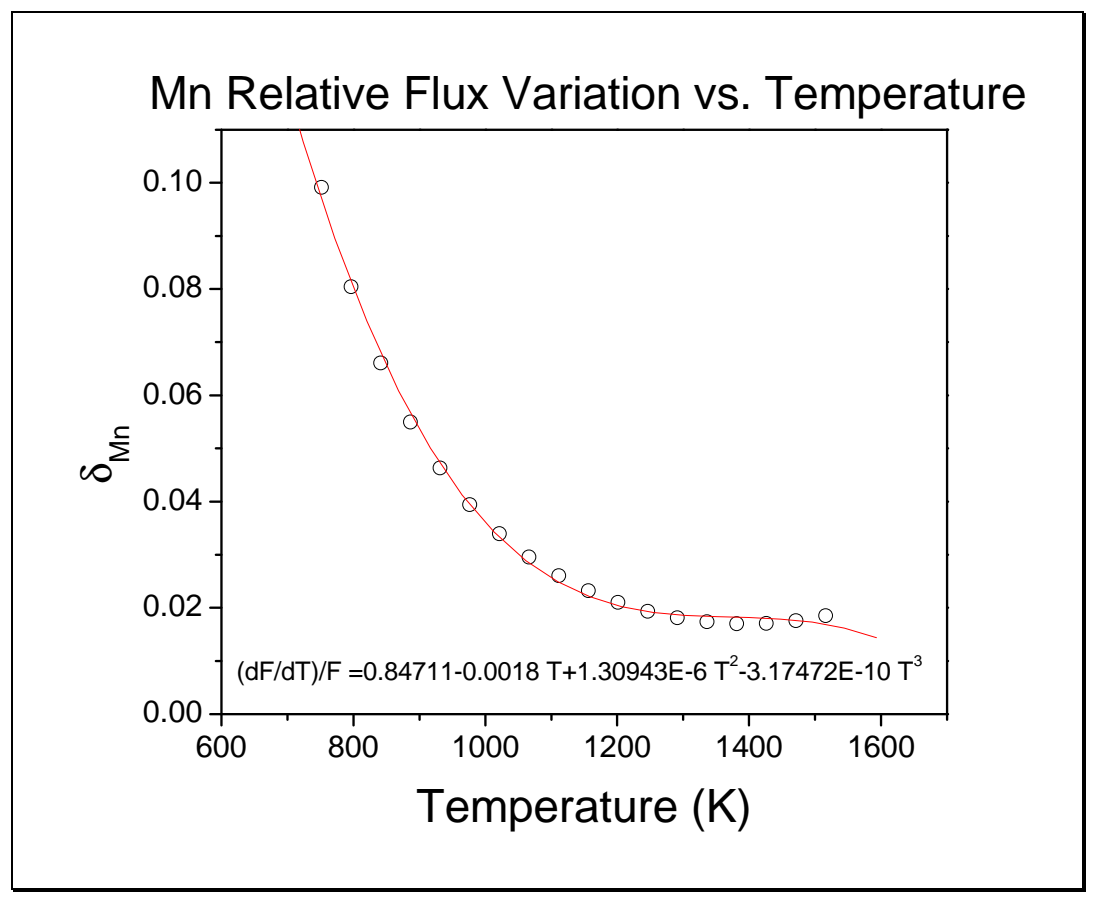

Figure 11: Mn relative flux versus temperature.

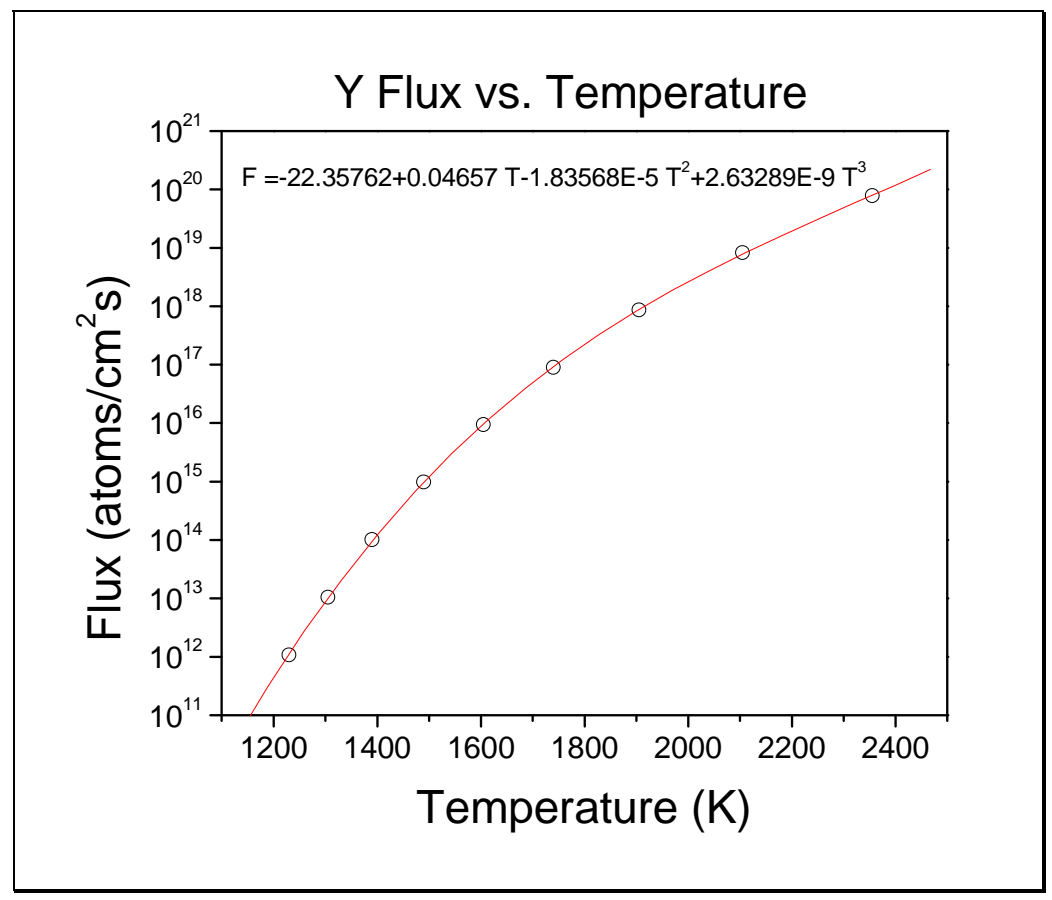

Figure 12: Y flux versus temperature. 


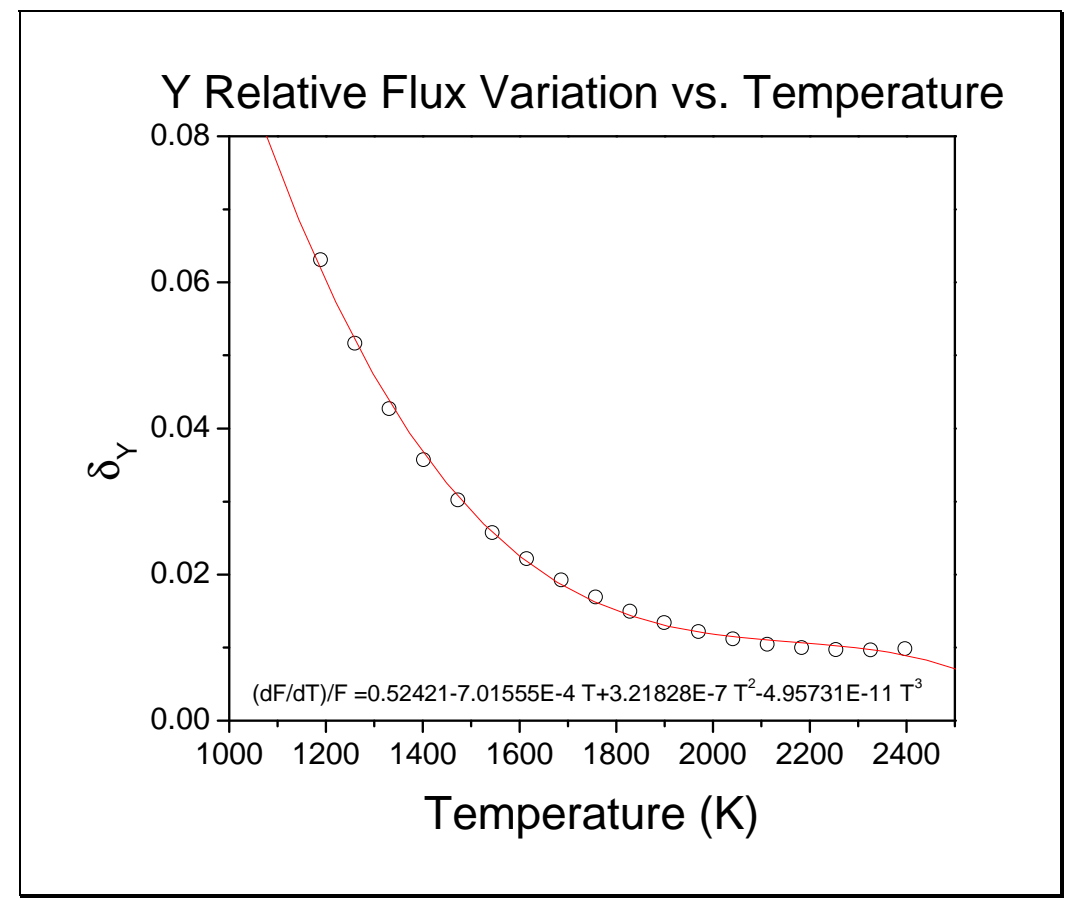

Figure 13: Y relative flux variation versus temperature.

Table 1: Stoichiometry variation due to $+/-.1^{\circ} \mathrm{k}$-cell temperature variation used in this study.

\begin{tabular}{|c|c|c|c|}
\hline Source & $\mathrm{T}(\mathrm{K})$ & $\mathrm{m}(\mathrm{g} / \mathrm{mol})$ & $\delta_{M n, Y}$ \\
\hline $\mathrm{Mn}$ & 1093 & 54.94 & .03 \\
\hline $\mathrm{Y}$ & 1667 & 85.91 & .02 \\
\hline
\end{tabular}

\subsection{RHEED}

Reflection high energy electron diffraction (RHEED) has become standard in ultra high vacuum deposition techniques, particularly MBE. A simplified schematic of a typical RHEED setup is shown in Figure 14 [43]. RHEED makes use of the small wavelength of relativistic electrons and small angle incidence in 
order to study the surface features of materials during deposition. Electron voltages used in RHEED are usually in the $5-40 \mathrm{keV}$ range. At this energy the wavelength of the electrons is approximated by [43]

$$
\lambda \approx \frac{12.247}{\sqrt{V\left(1+10^{-6} V\right)}}(\AA) .
$$

Where $\mathrm{V}$ in equation (2.4) is the accelerating voltage (volts). Electrons have a resulting wavelength in the range of $0.2-0.06 \AA$. The small angle of incidence results in a very small wavevector component normal to the sample surface, which reduces contributions to resulting diffraction from the planes parallel to the sample surface. Consequently, diffraction from in-plane lattice planes, or planes perpendicular to the sample surface, can be probed with high surface sensitivity. Penetration depth for electrons at this range of energy and angle of incidence is of the order of a few atomic layers [46]. If the sample surface is smooth and crystalline, diffraction can be observed from the sample according to Bragg's law (see section on $\mathrm{x}$-ray diffraction). 


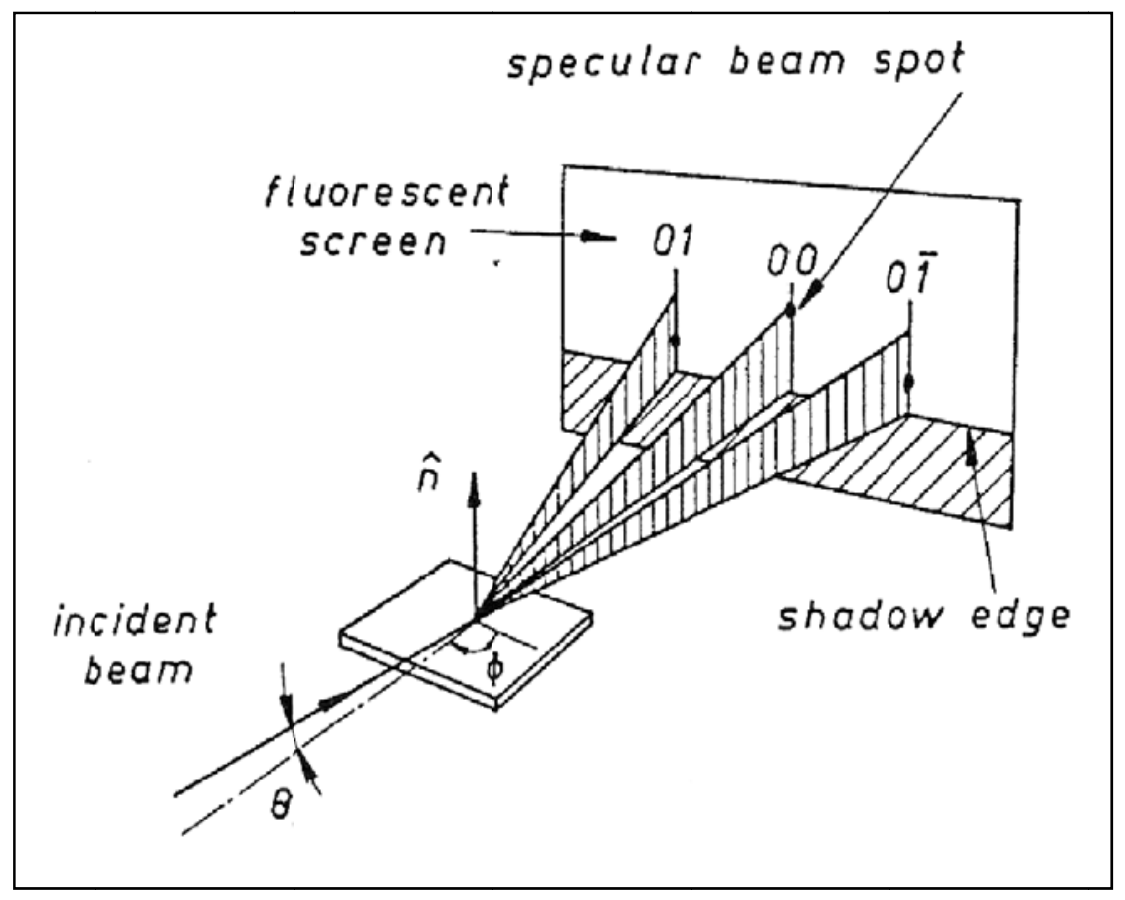

Figure 14: Simplified RHEED schematic. Incident beam angle is typically $1^{\circ}$. The fluorescent screen is located $180^{\circ}$ from the incident beam [43].

\subsection{X-Ray Diffraction}

Perhaps the most established and ubiquitously used technique in materials characterization is x-ray diffraction. X-ray diffraction was first discovered by Bragg in 1912. Since that time, x-ray diffraction has been used in almost every area of materials science. X-ray wavelengths, being on the scale of angstroms, are comparable to crystalline material lattice spacing. This makes x-ray diffraction a particularly useful technique for the characterization of thin film materials.

Two conditions are required for x-ray diffraction: 1) reflection and 2) interference [47]. Crystalline materials are composed of regular arrays of atoms that form atomic planes allowing for both above conditions. When a 
monochromatic source of x-rays impinges on a surface, regular lattice planes within the crystal result in high intensity reflections that can be observed to follow Bragg's law [48],

$$
2 d_{h k l} \sin \theta=\lambda,
$$

where $d_{h k l}$ is the spacing between lattice planes $(h k l), \theta$ is the angle of reflection with respect to the surface normal, and $\lambda$ is the $\mathrm{x}$-ray wavelength, respectively. By noting fundamental relations between $d_{h k l}$ and the reciprocal lattice structure [6], it can be shown that only reflections where the incident and reflected beams differ by a reciprocal lattice vector can result in diffraction. This can be stated in an alternate but equivalent form to Bragg's law

$$
\frac{\boldsymbol{s}-\boldsymbol{s}_{\mathbf{0}}}{\lambda}=\boldsymbol{H}_{h k l},
$$

where $\boldsymbol{s}$ and $\boldsymbol{s}_{\mathbf{0}}$ are unit vectors representing the reflected and primary beams and $\boldsymbol{H}_{h k l}$ is a reciprocal lattice vector perpendicular to the planes $h k l$ with length $2 \pi / d_{h k l}$. Figure 15 illustrates the geometry of diffraction. Figure 16 shows a useful construction known as the Ewald sphere. For any orientation of incident and reflected beams to produce diffraction, the point $h k l$ must fall on the sphere whose radius is $2 \pi / \lambda[49]$.

X-ray diffraction allows one to probe the crystal structure and quality of a material through a variety of scans. Figure 17 shows a four circle $\mathrm{x}$-ray diffractometer that can be used to perform all of the diffraction scans in this study. In order for any particular scan to be performed the sample has to be located at the center of rotation of the diffractometer. For all out-of-plane measurements, the center of rotation involves having the rotation angle $\chi=90^{\circ}$, where the reference 
or zero of $\chi$ is specified as zero when the normal to the sample surface is vertical. In-plane measurements require adjustments to either the angle $\chi$ away from $90^{\circ}$ or making appropriate adjustments to $\omega$ and $2 \theta$, such that the intended reflection can be measured. For a detailed derivation of the relationship between lattice structure, lattice constants, and diffraction angles see Ref. [47].

Figure 18 is a schematic of the reciprocal lattice points for a [001] oriented cubic crystal [43]. In order to determine crystal phases present and lattice constants for a material $\omega-2 \theta$ scans can be used. This scan configuration consists of recording the diffracted intensity as the incident beam is scanned through a range of angles while $\omega$ is fixed at half of the value of $2 \theta$. $\omega$ scans, also known as rocking curves, allow for an estimation of crystal quality. As $2 \theta$ is fixed, $\omega$ is scanned through a range of values around the ideal angle $2 \theta / 2$. This corresponds to rotating the Ewald sphere about the point (000), resulting in the spread in reciprocal space of the corresponding $2 \theta$ reflection. As long as the resolution of the instrument is kept constant, comparing the intensity and full width half maximum of a series of samples can allow for crystal quality comparison.

\subsection{X-Ray Reflectivity}

In the small incident angle regime, $x$-ray reflections from a material surface become very sensitive to the interfacial roughness, periodicity of the composition (such as in the case of multilayers), and layer thickness [50]. Examining the changing intensity of the reflected beam in the $\omega-2 \theta$ geometry produces 
oscillations commonly referred to as Kiessig fringes due to interference between reflections from different interfaces with Bragg peaks resulting from any large periodicity of the sample layers [51]. Using this information, Parratt developed a recursive method in which the electric field in each layer of a sample is modeled starting from the substrate, as the substrate is very thick and x-rays will not reflect from the substrate's bottom surface [52]. Several methods have also been developed to account for the roughness between layers. The most common practice was introduced by Névot and Croce where an exponential decay factor proportional to the square of the vertical roughness was introduced at each interface [53]. Parratt's algorithm was used to determine the roughness and thickness for each of the samples examined in this study. 


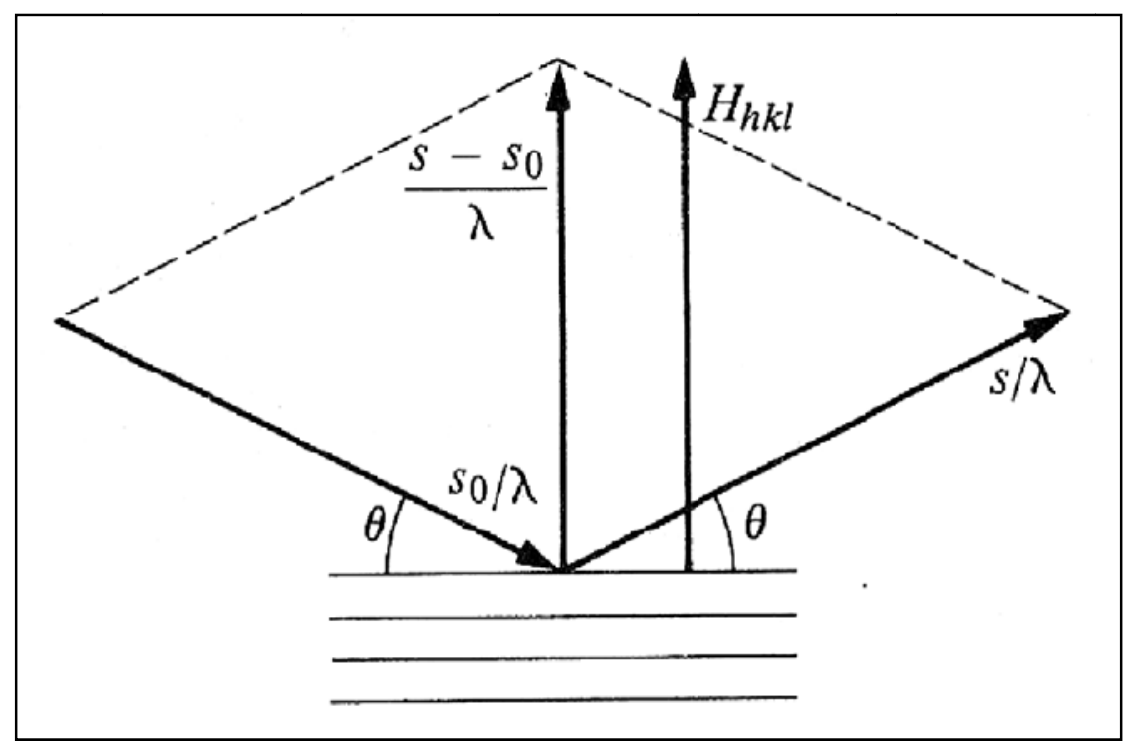

Figure 15: Illustration representing x-ray diffraction from a periodic lattice. $s$ and $\boldsymbol{s}_{0}$ are the scattered and incident X-ray unit vectors, respectively. $\boldsymbol{H}_{h k l}$ is a vector perpendicular to the lattice planes $h k l$ with length $2 \pi / d_{h k l}[47]$.

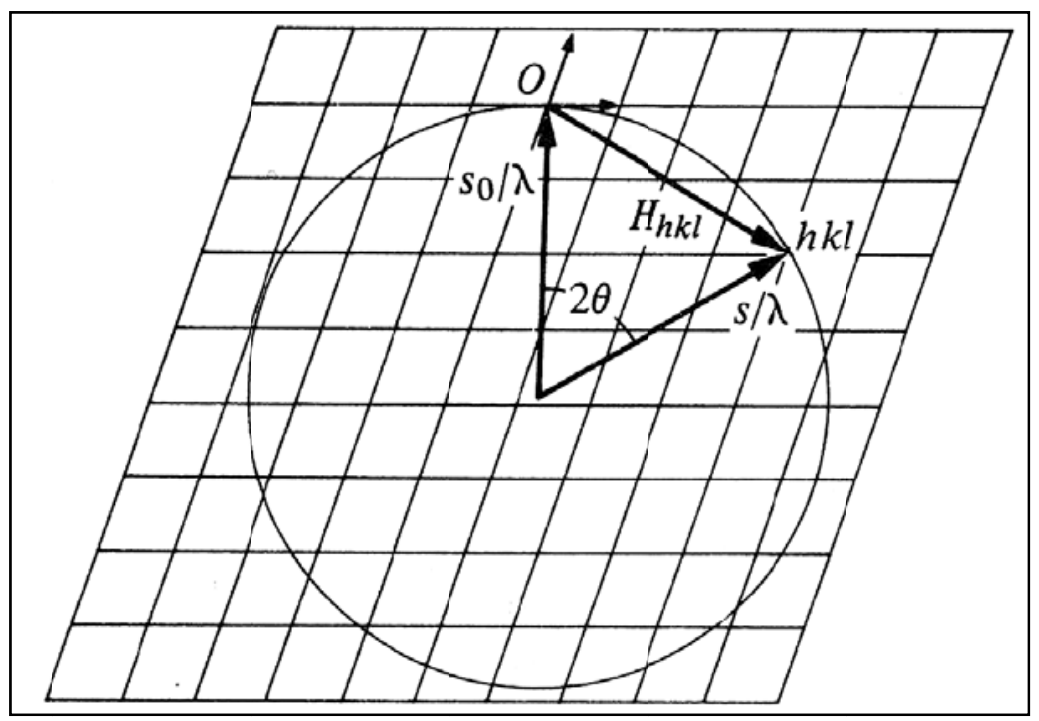

Figure 16: Two-dimensional representation of the Ewald sphere construction. The Bragg condition is satisfied for any point $h k l$ that falls on the sphere [47]. 


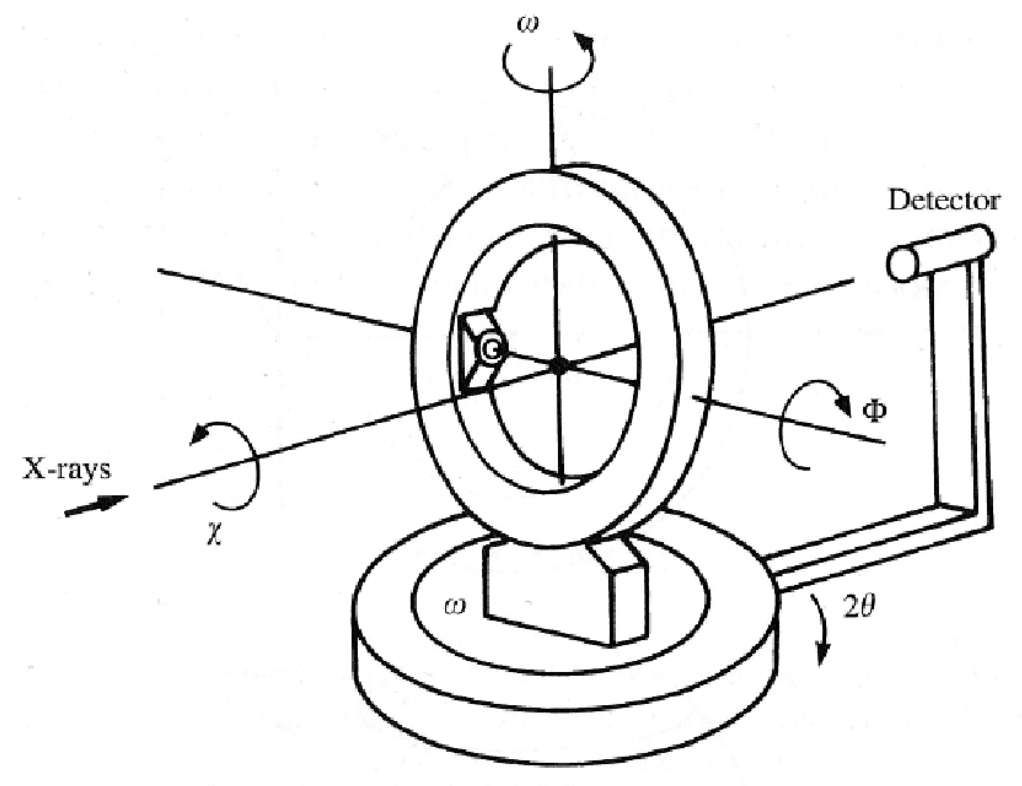

Figure 17: Four circle x-ray diffractometer [49].

+ reciprocal lattice points

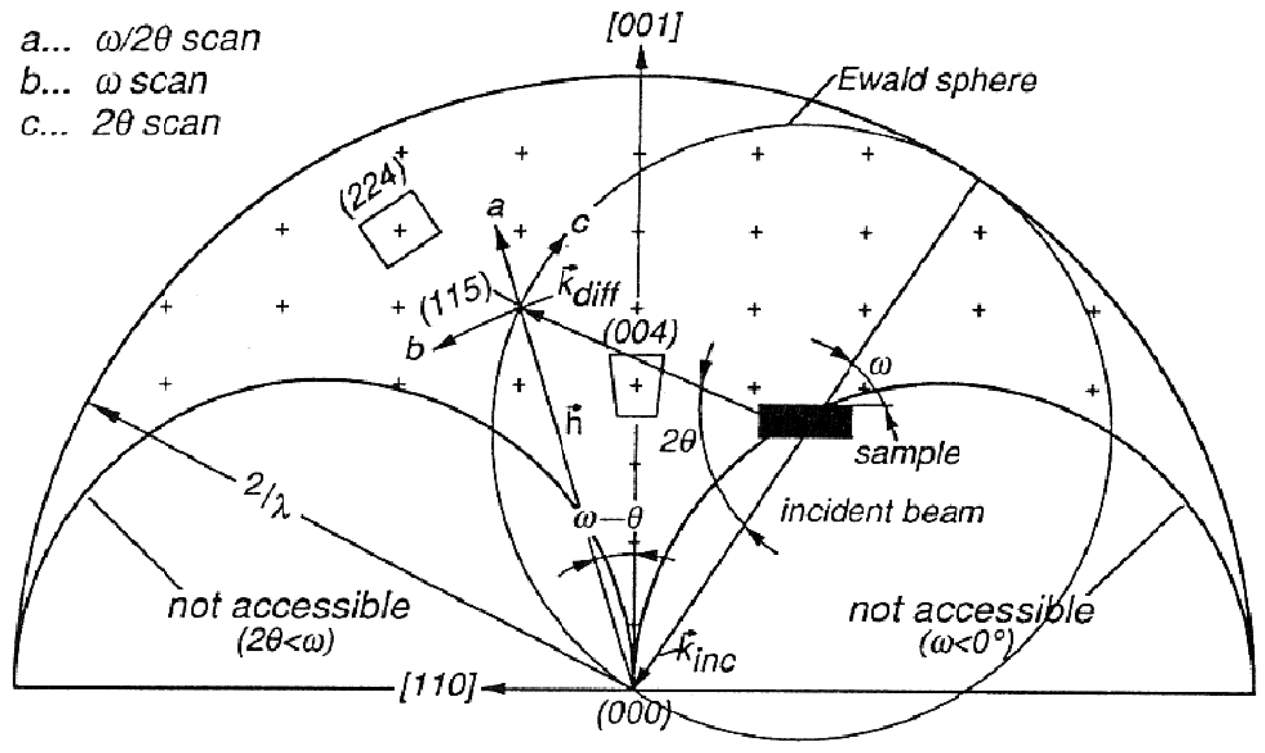

Figure 18: Schematic of the reciprocal lattice for a [001] oriented cubic crystal. The Ewald construction is shown for a (115) reflection with $a, b$, and $c$ corresponding to $\omega-2 \theta, \omega$, and $2 \theta$ scans, respectively [43]. 


\subsection{Atomic Force Microscopy}

Atomic force microscopy (AFM), while being a powerful technique that has spawned a whole variety of microscopy fields, is in principle one of the simplest systems used today in materials science. In practice, AFM is used to determine surface morphology by two feedback methods, commonly referred to as contact mode and tapping, or intermittent contact, mode. AFM was originally developed using contact mode, where a cantilever with an approximately $10 \mathrm{~nm}$ radius of curvature tip is placed in contact with a surface and a laser is reflected off the back of the cantilever onto a diode array. This diode array can then be used as a feedback mechanism to keep the force, or displacement, between the cantilever and the sample constant. Either the sample or the cantilever is conventionally mounted on a calibrated piezoelectric material with which a given applied voltage corresponds to a known vertical displacement. The amount by which the system has to adjust the displacement of the cantilever or sample to maintain equilibrium can be recorded and a topographic map of the surface can be obtained. For hard samples without strong surface adhesion, this method can produce high quality surface maps. This method has several drawbacks, however. If the sample is soft, the cantilever may become contaminated and begin to produce aberrant data. Soft samples can also easily be damaged by the cantilever.

Tapping mode AFM was developed as a means to image samples while limiting surface damage and providing improved signal to noise ratio. In contrast to contact mode, feedback for tapping mode is provided by driving the cantilever 
at a fixed frequency and monitoring the RMS voltage of the laser reflection produced on the diode array via a lock-in amplifier. By using an $\mathrm{AC}$ as opposed to DC detection technique, and by reducing tip sample forces involved, image quality can be increased while minimizing sample damage and extending cantilever lifetime. Typical contact and tapping mode AFM setups are shown in Figure 19.
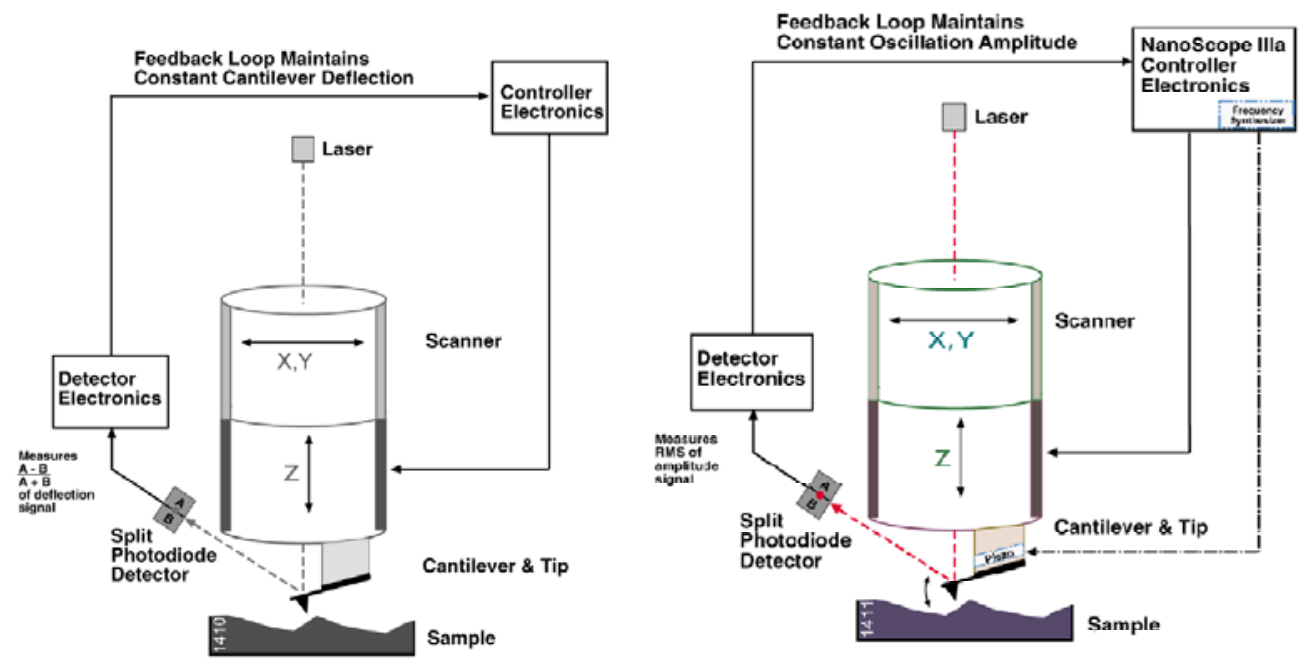

Figure 19: AFM contact (left) and tapping (right) mode operational schematics

[54].

\subsection{Electrical Characterization}

The primary goal of any ferroelectric material research is to determine whether or not the material can be developed in a manner suitable for device applications. In order to accomplish this goal, a variety of experimental techniques have been developed to measure everything from the onset of phase transitions to electrical breakdown under high electric fields. The first method to 
measure the polarization of sample was devised by Sawyer and Tower in 1930 [55]. This method is still in use to this day, but has limitations and can result in erroneous estimations of polarization. Figure 11 shows two common methods to measure polarization as a function of applied voltage $(P-V)$. Figure 20(a) corresponds to the Sawyer Tower method. One drawback associated with this method is the requirement for a reference capacitor at least 100 times the capacitor being tested; otherwise, as the voltage across the reference capacitor increases the voltage across the test device decreases (referred to as the back voltage effect). The back voltage effect then causes a need for a different reference capacitor for each sample under test. When testing small capacitance samples, parasitic capacitance from the cables and measurement circuitry, being in parallel with the reference capacitor, can dominate the measurement, giving spurious results. Also, the resistance of the measurement equipment is in parallel with the reference capacitor and as such acts as an $\mathrm{RC}$ circuit. This results in discharging and erroneous results when low test frequencies are used [5].

A modification to the Sawyer Tower method, shown in Figure 20(b), is known as the virtual ground method. This circuit uses a current to voltage converter connected to a feedback resistor which then connects to the inverting input of an operational amplifier. The non-inverting input of the amplifier is connected to ground. Current is integrated over a fixed amount of time and converted to a measurement of polarization. This method removes the back voltage effect and lowers the magnitude of parasitic effects due to cable capacitance and impedance. 
Leakage measurements are generally performed using a circuit in which a DC voltage is applied to the device under test and the current flowing through the circuit is subsequently measured after some specified delay or "soak" time. Several quantities or variations on this measurement can be performed in which the soak time, measurement time, and preset pulse configuration are modified. Figure 21 and Figure 22 illustrate the configuration used to measure the samples in this work [54]. Figure 21 and Figure 22 show the single data point measurement cycle in the $I-V$ curve and the unswitched pulse configuration used to capture the complete measurement cycle, respectively. Unswitched preset pulse polarity was used in order to remove any switching components to the leakage current.

(a)

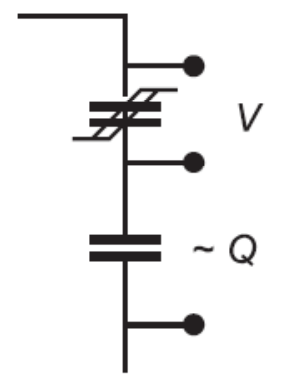

(b)

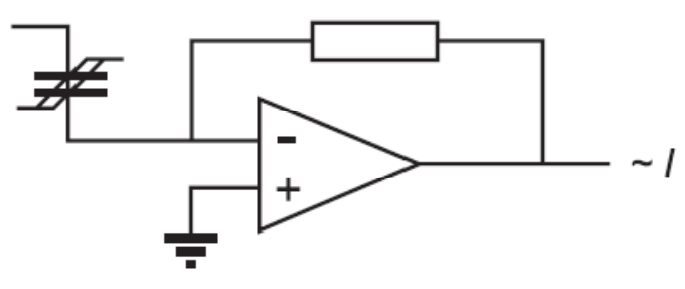

Figure 20: Sawyer-Tower circuit (a) and virtual ground method (b) testing techniques for ferroelectric polarization [5]. 


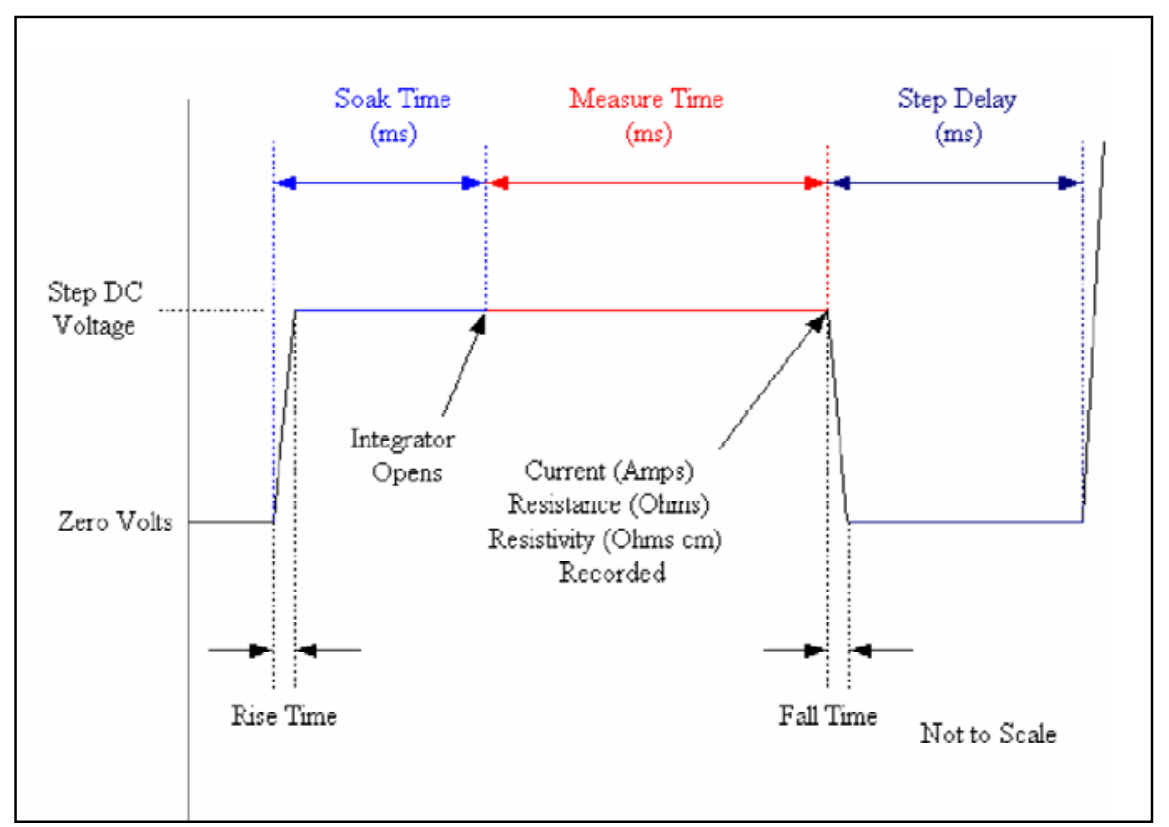

Figure 21: Individual $I-V$ data point measurement cycle [56].

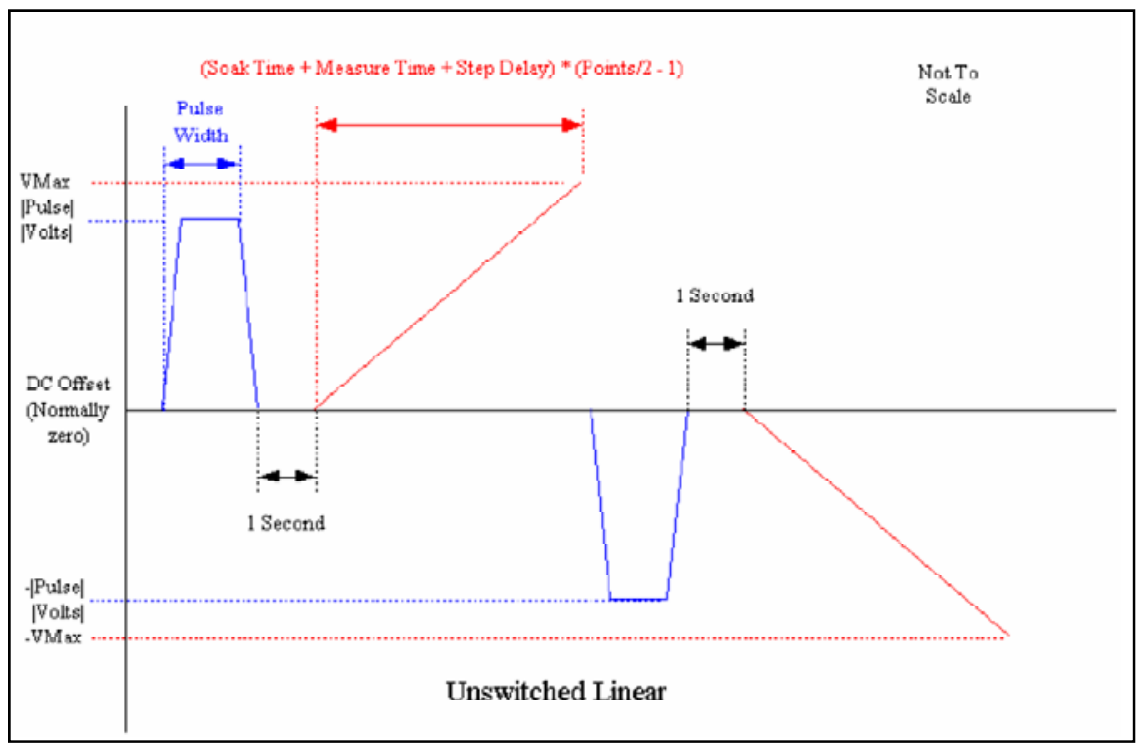

Figure 22: Unswitched linear $I-V$ measurement diagram [56]. 


\section{Chapter 3: Experimental Procedure}

\subsection{Sample preparation and deposition}

Commercial GaN templates were degreased for 5 minutes in separate acetone and methanol baths preceding an acid etch in $\mathrm{HCl}$ for 5 minutes to remove any oxide layer formation. Subsequently, the sample was placed face down on a clean piece of aluminum foil which was heated on a hot plate to roughly $220{ }^{\circ} \mathrm{C}$. Indium tin eutectic purchased from Alpha Aesar was then spread evenly over the back of the sample while the sample was firmly held in place using downward pressure from a pair of tweezers. The sample was then mounted to a 2 inch silicon wafer that was also placed on the hot plate. After the sample had been transferred to silicon, the sample was pushed along one corner while being held by a clean pair of tweezers at another corner. This motion allowed for the eutectic to spread to a thin layer and form a strong bond between the sample and silicon. This method did require, however, careful attention in order to not contaminate the sample during the mounting process.

Silicon was used as the sample holder to provide a thermally uniform surface with strong sample adhesion. Earlier attempts to use thinly cut stainless steel proved unsuccessful. Stainless steel, being a poor thermal conductor, had to be cut quite thin in order for the sample to heat uniformly. This eventually led to problems associated with warping of the sample holder under the high substrate temperatures needed to produce crystalline samples. Alumina was also attempted 
as a sample holder. Being an oxide ceramic, it could easily withstand the temperatures involved. Unfortunately, with repeated growths the alumina holders began to resist surface wetting by the eutectic used to bind the substrate, resulting in non-uniform sample heating. Also, the indium in the eutectic began to contaminate the samples during growth as it evaporated from behind the sample (see Chapter 5). Etching and baking of the alumina sample holders did not improve wetting, so their use was abandoned.

After the sample was mounted to a silicon wafer it was placed into a load lock chamber and pumped down to roughly $10^{-6}$ Torr using a turbo-molecular pump. The load lock connected a series of isolated chambers through a cluster tool equipped with an ion pump capable of maintaining the cluster at roughly $10^{-9}$ Torr. Each attached chamber was dedicated to deposition of a specific class of material, i.e. metals, nitrides, and oxides. Next, the sample was introduced into the chamber dedicated for oxide growth. The chamber was constructed by SVT Associates and was equipped with a Pfeiffer TPU 1201P turbo-molecular pump, Gamma Vacuum TiTan500T ion pump, Veeco K-cells for Y and Mn, and an SVT oxygen plasma source. Background pressure in the chamber was typically $10^{-10}$ Torr. Each source, including the plasma, was equipped with a shutter to prevent sample contamination and to allow layer-by-layer growth. Prior to deposition both the $\mathrm{Y}$ and $\mathrm{Mn}$ sources were calibrated using an Inficon quartz crystal microbalance (QCM) controlled via an IC-5 deposition controller placed at the sample position. Flux calibration via QCM is generally regarded as being accurate to within approximately $5 \%$. Once stable flux measurements were 
recorded by the QCM, shutter times for $\mathrm{Mn}$ and $\mathrm{Y}$ were determined that would provide $9.719 \times 10^{14}$ atoms $/ \mathrm{cm}^{2}$, the surface site density needed to fill each sublattice of YMO.

After the K-cells were calibrated, the sample was heated to $700{ }^{\circ} \mathrm{C}$. This temperature was chosen in order to minimize damage to the GaN surface and attempt to prevent oxidation. Sample temperature was controlled via a Eurotherm 2408 temperature controller with a Sorenson DC S60-18E power supply using feedback from a backside mounted C-type thermocouple. Oxygen flow into the chamber was started at a rate of $0.2625 \mathrm{sccm}$. Power to the plasma source was then raised in $50 \mathrm{~W}$ increments until the forward power reached $200 \mathrm{~W}$. If the plasma had not ignited on its own by this time, the oxygen flow was briefly restricted using a mechanical valve between the oxygen flow controller and the plasma source. When this valve was re-opened, a burst of oxygen into the plasma cavity would ignite the plasma. At this point the pressure in the chamber was roughly $2 \times 10^{-6}$ Torr.

Once the plasma had been ignited, two unit cells of YMO were deposited on the substrate via layer-by-layer deposition (beginning with $\mathrm{Y}$ ) and then the substrate shutter was closed. At this stage, RHEED did not indicate that the material was crystalline. Higher temperatures were required for the material to crystallize into the desired layered structure. The sample temperature was next raised to $850{ }^{\circ} \mathrm{C}$, where it was annealed for roughly 4 minutes. Figure 23 demonstrates the shuttered RHEED oscillations beginning with the first annealing phase. The shuttered oscillations indicate that the sample may be Y rich, as the 
positive slope portions of the trace were during the $\mathrm{Y}$ deposition [57]. Samples with deposition temperatures higher than $850{ }^{\circ} \mathrm{C}$ were annealed at the corresponding growth temperature. After this time RHEED streaks would routinely appear. The temperature would then be changed to the temperature for that particular growth. At this point, two more unit cells would be deposited and then the sample would be annealed with the substrate shutter closed to the oxygen. The RHEED pattern was monitored to determine when to deposit more material. When the RHEED pattern was again clear and streaky, one more annealing phase would be done after the deposition of five unit cells. Again, the RHEED would be monitored to determine when to proceed with the deposition. After the last anneal, material would be deposited on the sample in ten to eleven unit cell increments until the sample was fifty unit cells thick.

Lastly, the sample was allowed to cool in oxygen until it was below $200{ }^{\circ} \mathrm{C}$. The oxygen/plasma was then stopped. Once the sample reached room temperature, it was removed from the chamber and decoupled from the silicon wafer by heating the wafer with a hot plate at $220{ }^{\circ} \mathrm{C}$. 


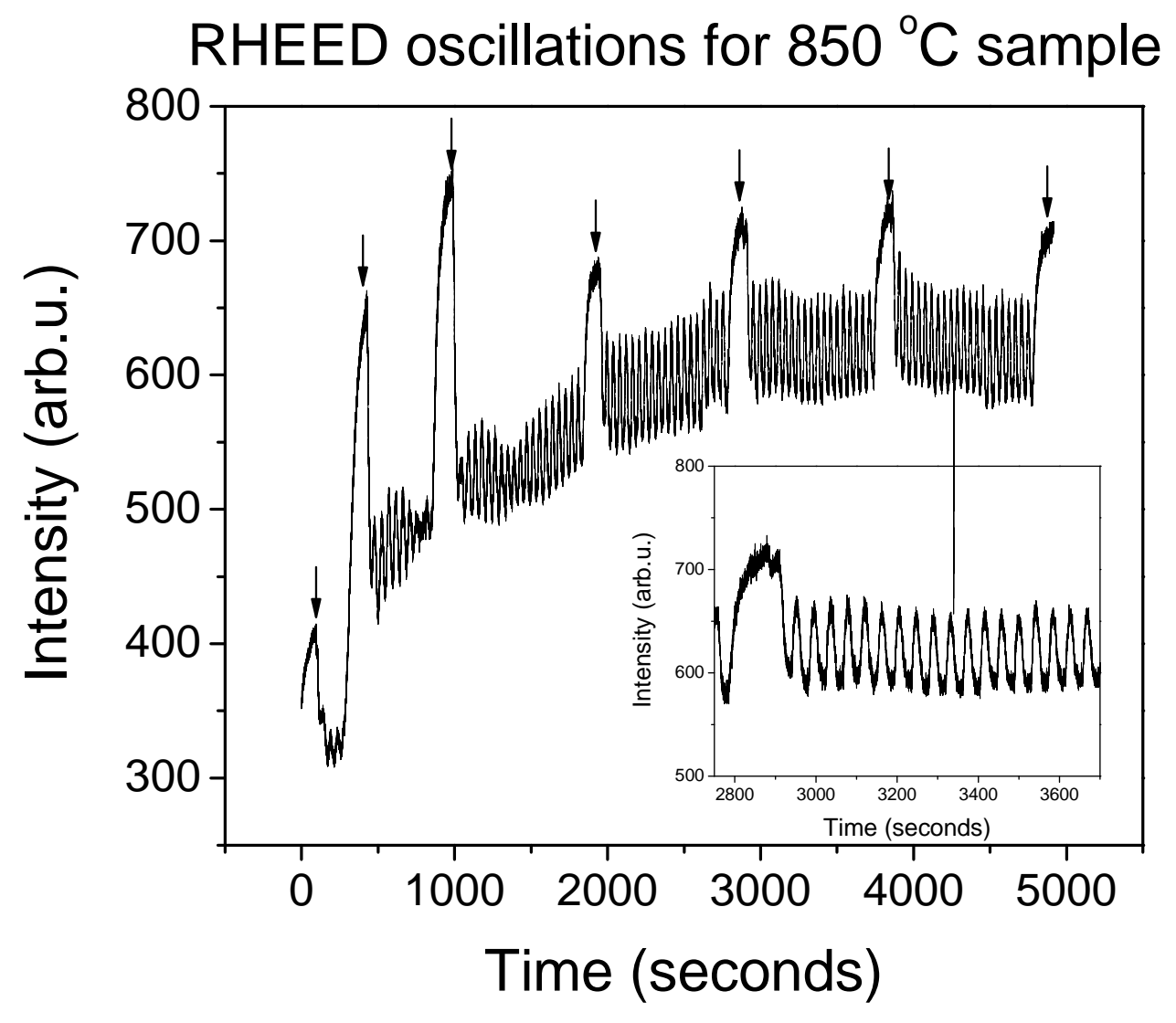

Figure 23: Shuttered RHEED oscillation captured for $850{ }^{\circ} \mathrm{C}$ sample. Arrows indicate anneal periods. The inset shows magnified region from 2750 to 3650 seconds. Each period corresponds to half a unit cell, with Y on the positive slope and $\mathrm{Mn}$ on the negative slope.

\subsection{Structural analysis}

\subsubsection{RHEED}

RHEED images of each sample were taken prior to growth, i.e. the GaN substrate and subsequent to growth. During growth RHEED images were captured for samples that displayed observable RHEED patterns. All images 
were captured using a k-Space Associates data acquisition system and Staib instruments RHEED gun capable of $30 \mathrm{keV}$ and $100 \mu \mathrm{A}$. Electron emission was fixed at $18 \mathrm{keV}$ and $\sim 0.6 \mu \mathrm{A}$ for each of the samples grown in this study.

\subsubsection{X-ray diffraction}

X-ray diffraction measurements were performed using a Rigaku RU-300 Cu $\mathrm{K} \alpha$ (wavelength $1.5418 \AA$ ) x-ray source with a four-circle diffractometer. A bentcrystal graphite monochromator was used to select the $\mathrm{Cu} \mathrm{K \alpha}$ radiation and to focus the beam at the sample position. Prior to measurements, the system was aligned to the $\mathrm{GaN}(004)$ reflection. This peak provided a strong reflection with a reproducible angle from sample to sample with which to calibrate $2 \theta$. Out-ofplane $\omega-2 \theta$ scans were performed on each sample to determine crystal phases present, c-axis lattice constants, and to evaluate crystal quality. Rocking curves were also recorded for the (002) peaks in order to compare the crystal quality from sample to sample.

In-plane measurements of the GaN (104) and YMO (106) or YMO (112) were performed to determine if the samples were epitaxial and with what crystallographic orientation with respect to the substrate. The YMO (106) or YMO (112) peaks were also used to determine the in-plane lattice constant for each sample.

Note that some diffraction scans included extraneous peaks due to higher energy bremmstrahlung radiation at multiple wavelengths of the $\mathrm{Cu} \mathrm{K} \alpha$ radiation. 
This issue was later resolved by filtering the energy of the x-ray pulses by lowering the upper energy limit in the x-ray detector's preamplifier.

\subsubsection{X-ray reflectivity}

X-reflectivity measurements were performed using the same x-ray source used for x-ray diffraction. A separate 2-circle diffractometer setup optimized for reflectivity measurements was used in order to obtain improved resolution at low $\mathrm{x}$-ray incidence angle. The resolution of the instrument was $0.003 \AA^{-1}$. X-ray reflectivity data was analyzed using the free program GenX [58]. GenX uses Parratt's recursion algorithm along with a genetic parameter minimization scheme. Sample thickness and interface roughness were extracted from the data for each sample. Included in the fitting model, represented by Figure 24, was a $\mathrm{GaO}$ layer formed at the interface between the GaN and YMO. TEM measurements performed on a sample grown under similar conditions to the samples in this study revealed this interfacial layer, as illustrated in Figure 25. 


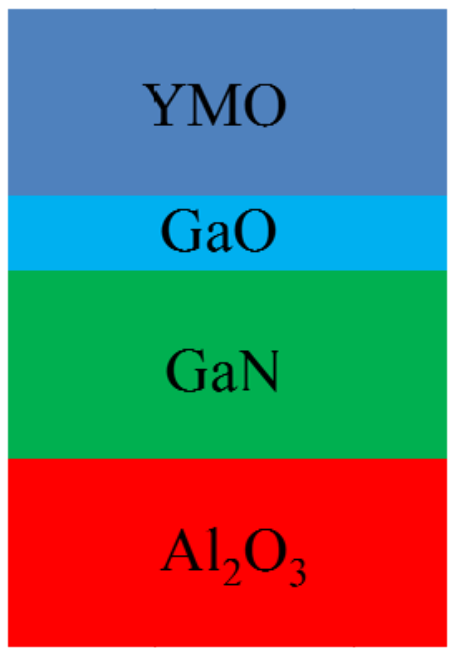

Figure 24: Illustration of fitting model used for x-ray reflectivity. Each layer is not drawn to scale and roughness between each interface is not indicated.

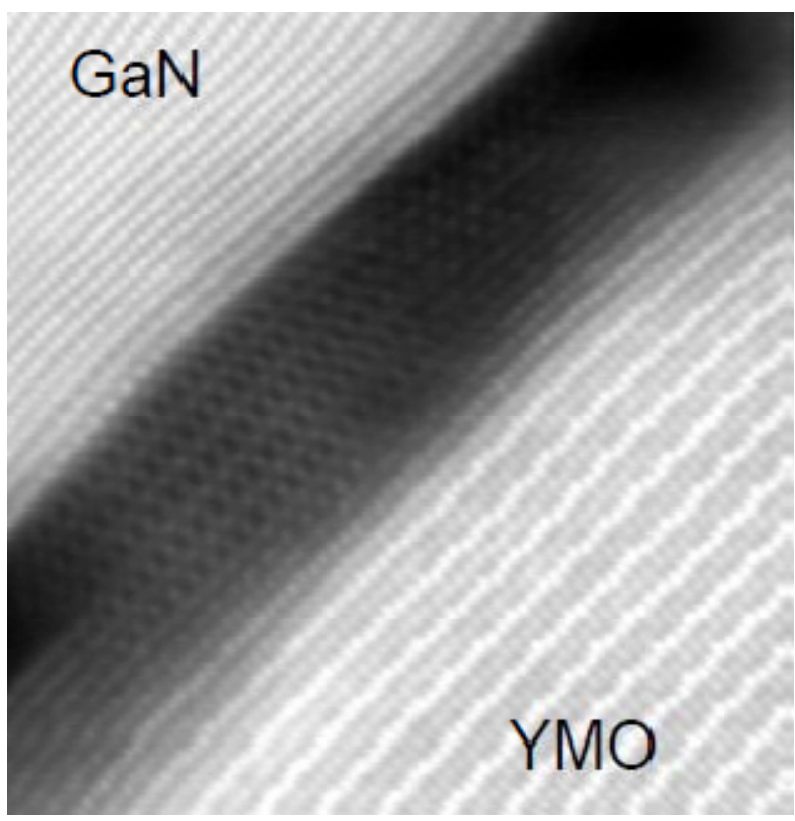

Figure 25: TEM image of the interface of a sample grown under similar conditions to the samples discussed in this study.

\subsubsection{AFM}

Topographic images of each sample were taken using a Veeco Multimode scanner controlled by a Nanoscope IIIa controller. Tapping mode imaging was 
used with NanoWorld Pointprobe NCHR cantilevers. NCHR cantilevers are 125 $\mu \mathrm{m}$ long, have a spring constant of $\sim 42 \mathrm{~N} / \mathrm{m}$, and have a typical tip radius of curvature of less than $8.0 \mathrm{~nm}$.

\subsection{Electrical Characterization}

Electrical measurements were performed using a Radiant Technologies LC ferroelectric tester system. Capacitor structures were made with Pt top contacts of area $400 \mu \mathrm{m}^{2}$ and thickness of $150.0 \mathrm{~nm}$. The Pt contacts were applied using ebeam lithography and sputtering followed by a lift-off procedure in acetone. Sputtering was performed in a chamber with a background pressure of approximately $5 \times 10^{-8}$ Torr. Argon gas with a flow of $5 \mathrm{sccm}$ and pressure of 3 mTorr was used to generate the sputtering plasma with a current of $50 \mathrm{~mA}$. In order to make contact to the bottom electrode, the GaN substrate, the sample was scratched at two opposing corners and indium was soldered to each area. Indium was used, as indium provides an ohmic contact to GaN. Two contacts were made to the GaN to verify that the indium was in contact with the GaN.

Each sample was mounted to glass using adhesive. The sample and glass were then mounted to a conducting metal disc that was grounded directly to the testing system. Two micro-positioners were used with tungsten filament probes to make contact to both the indium and the Pt contacts. Coaxial cable was used between the tungsten filaments and the testing system in order to reduce

measurement noise. An optical microscope, also grounded directly to the testing system, was used to allow for the positioning of the filament to the top Pt contact. 
Polarization as a function of applied voltage $(P-V)$ measurements were performed for a series of maximum voltages, typically $0.5 \mathrm{~V}$ to $1 \mathrm{~V}$. All $P-V$ data were captured using a preset loop with a delay of $10 \mathrm{~ms}$ and a cycle time of $10 \mathrm{~ms}$. Current as a function of applied voltage $(I-V)$ measurements were performed with $10 \mathrm{~ms}, 5 \mathrm{~V}$ preset pulses in an unswitched. $I-V$ data capture was done with a soak time $100 \mathrm{~ms}$, delay time of $100 \mathrm{~ms}$, and measurement time of $1 \mathrm{~s}$. The soak and measurement times were chosen to remove displacement and switching current contributions from the results. 


\section{Chapter 4: Results and Discussion}

\subsection{Structural results}

\subsubsection{RHEED}

Beginning with the lack of crystalline features of any kind in the sample grown at $750{ }^{\circ} \mathrm{C}$ (Figure 26 right panel), there was a noticeable improvement in the RHEED images as sample growth temperature increased. All samples above $750{ }^{\circ} \mathrm{C}$ showed crystalline features without the inclusion of polycrystalline (rings) or 3-dimensional (3D) growth (transmission spots) phases. As the growth temperature exceeded $800{ }^{\circ} \mathrm{C}$, the RHEED became sharper and surface reconstructions more pronounced. The sample grown at $900{ }^{\circ} \mathrm{C}$ displayed the sharpest diffraction streaks and strongest reconstruction features of all the samples (Figure 27). Images for samples $775{ }^{\circ} \mathrm{C}$ through $875{ }^{\circ} \mathrm{C}$ are shown in Appendix A1. 


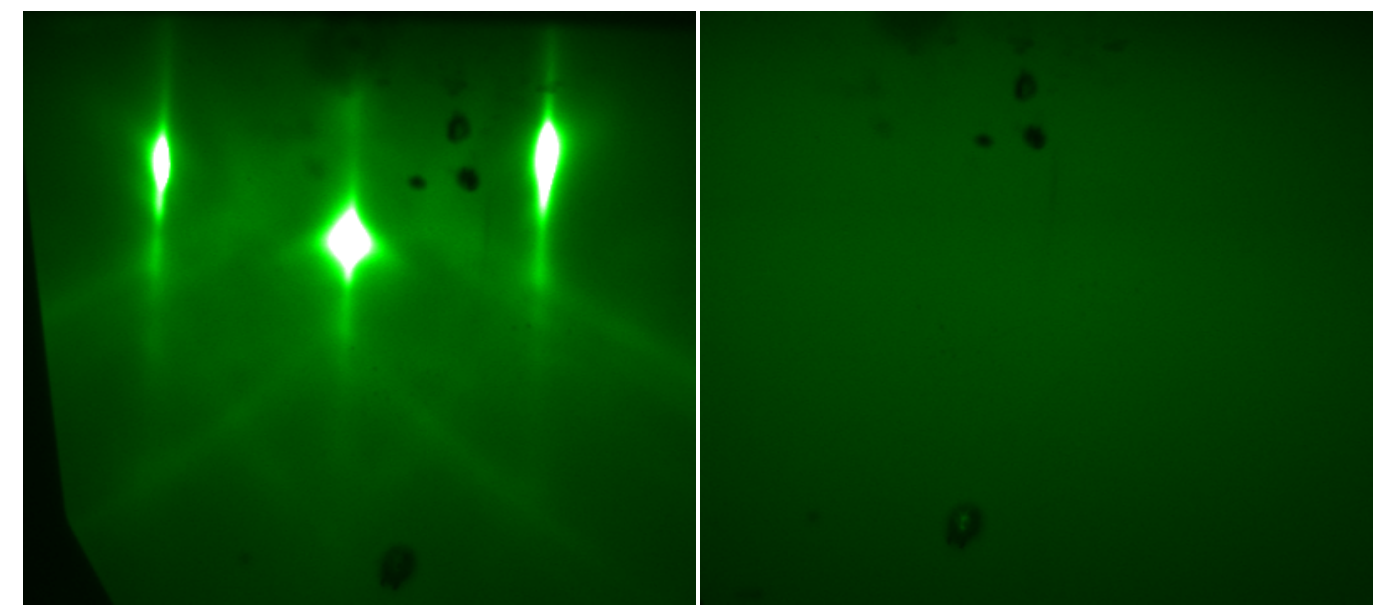

Figure 26: RHEED for sample grown at $750{ }^{\circ} \mathrm{C}$. (Left) GaN before deposition. (Right) Post deposition images show no clear crystalline features.

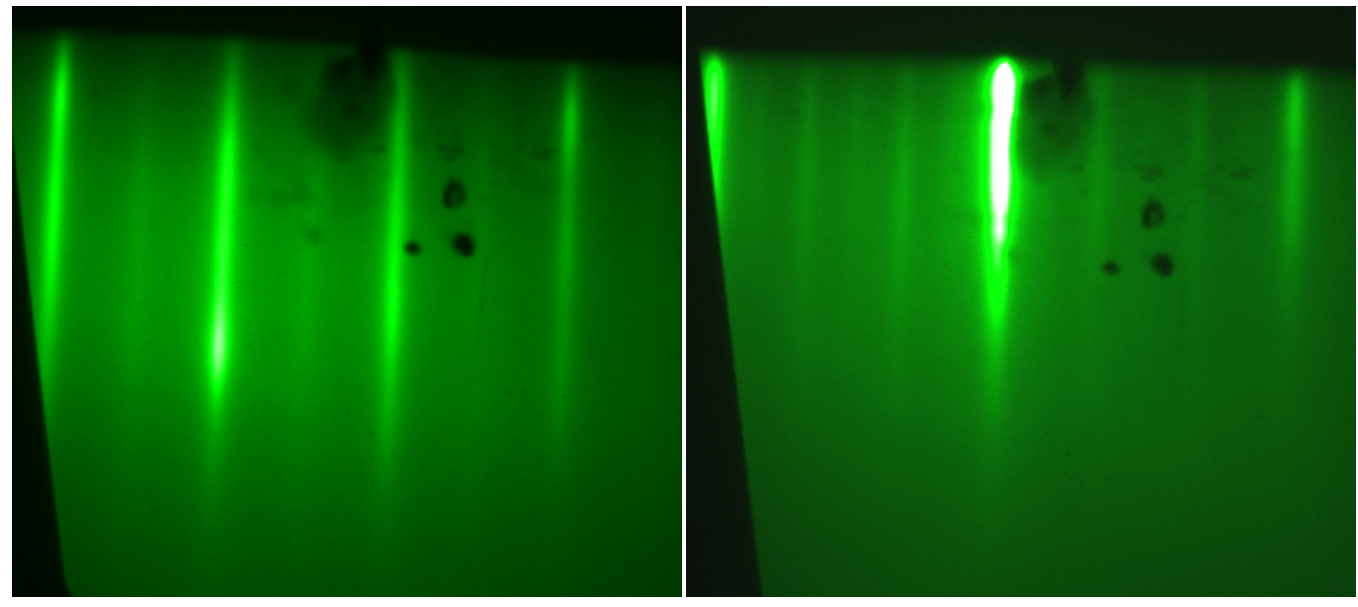

Figure 27: RHEED for sample grown at $900{ }^{\circ} \mathrm{C}$. (Left) RHEED (110) lattice reflection. (Right) RHEED (1-10) lattice reflection.

\subsubsection{AFM}

AFM revealed that the sample surfaces had a distinct dependence upon deposition temperature. In the case of the sample grown at $750{ }^{\circ} \mathrm{C}$, the surface was well matched to the GaN surface morphology before deposition, as shown in Figure 28. This resulted from the amorphous/polycrystalline nature of the 
sample. As the atoms deposited on the surface they arranged themselves not epitaxially, but with a random configuration. Figure 30 shows the sample root mean square (RMS) roughness as a function of deposition temperature, as determined via AFM and x-ray reflectivity. Both AFM and x-ray reflectivity indicated that the sample roughness increased with temperature. Visible in the AFM images are features that may correspond to clumping of material due to nonstoichiometric deposition. As the deposition temperature increased, the area of the surface occupied by these features increased. These features are visible in Figure 29, the AFM surface image of the $900{ }^{\circ} \mathrm{C}$ sample. Surface roughening may indicate that the increased energy due to the higher temperatures allowed for greater surface migration and, consequently, coalescence of large surface features. This trend may also result from a change in Mn incorporation due to desorption, as the temperature of deposition was comparable to the Mn k-cell temperature. AFM images of samples $775{ }^{\circ} \mathrm{C}$ through $875{ }^{\circ} \mathrm{C}$ are shown in Appendix A.2.

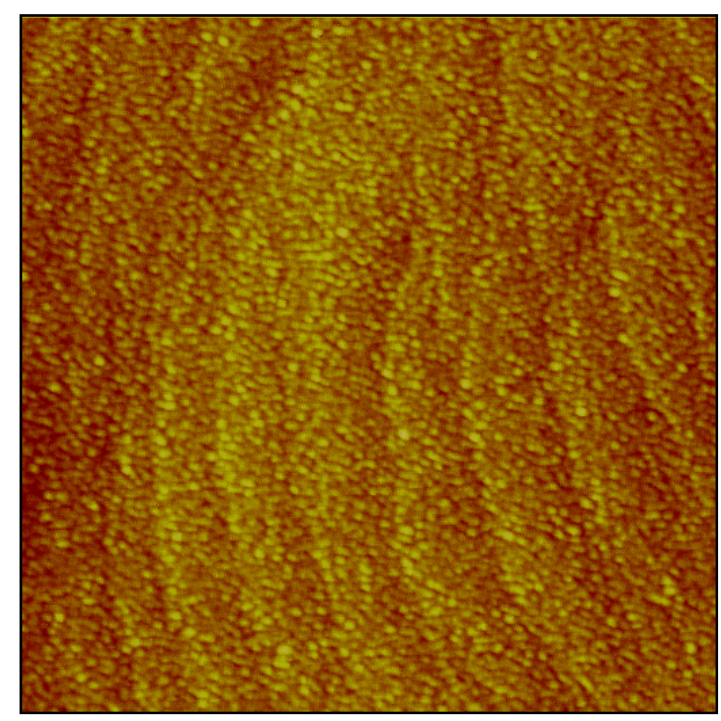

Figure 28: $1 \mu \mathrm{m}^{2} \mathrm{AFM}$ image of sample grown at $750{ }^{\circ} \mathrm{C}$. RMS roughness was estimated to be $3.4 \AA$. 


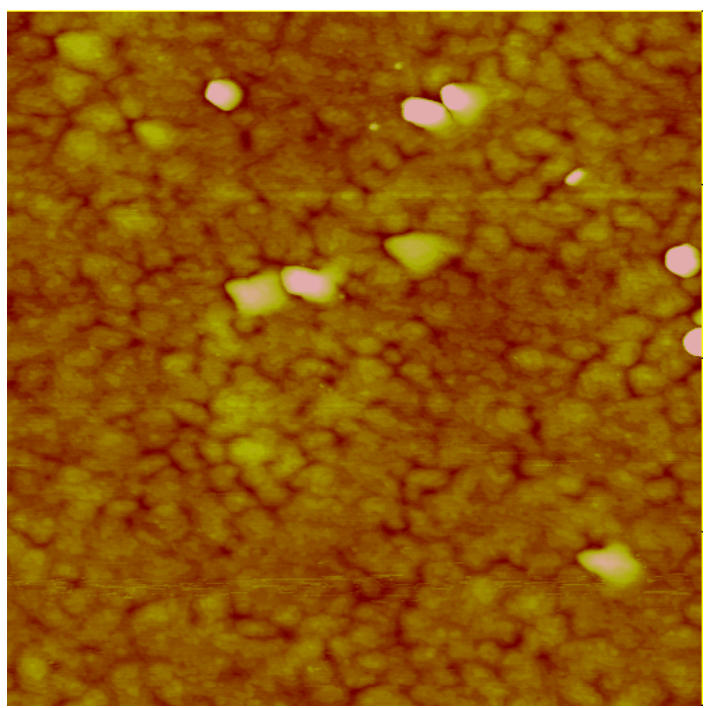

Figure 29: $1 \mu \mathrm{m}^{2} \mathrm{AFM}$ image of sample grown at $900{ }^{\circ} \mathrm{C}$. RMS roughness was estimated to be $9 \AA$.

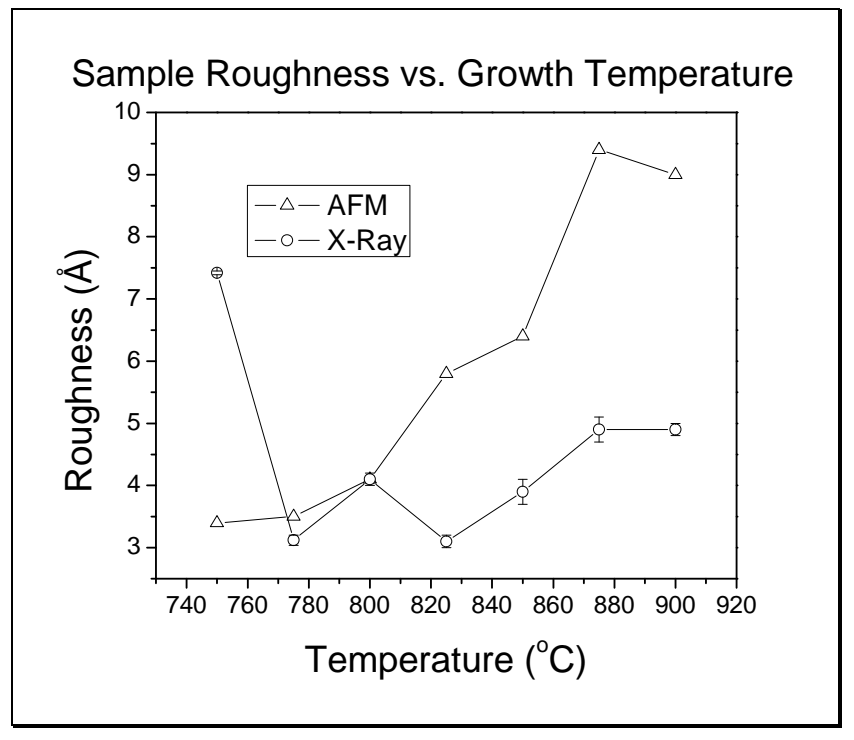

Figure 30: Sample roughness versus growth temperature. 


\subsubsection{Out-of-Plane X-Ray Diffraction}

All sample temperatures, neglecting the $750{ }^{\circ} \mathrm{C}$ sample, displayed the expected $(00 l)$ reflections. Figure 31 shows the out-of-plane x-ray diffraction of the $900{ }^{\circ} \mathrm{C}$ sample as an example of the captured data. The results for the other sample temperatures can be viewed in Appendix A.3. Samples with deposition temperatures of $825{ }^{\circ} \mathrm{C}$ and above also showed two peaks that could not be identified, located at approximately $2 \theta=30.6^{\circ}$ and $63.8^{\circ}$. Figure 32 shows both of these peaks for the sample at $900{ }^{\circ} \mathrm{C}$. An extensive database search of possible reflections and/or materials that could possibly result in these peaks did not prove fruitful. Table 2 shows the YMO (006) peak centroids and corresponding c-axis lattice parameters as a function of growth temperature. C-axis lattice parameter did not correlate or show any specific trend with growth temperature. An example fit of the $775{ }^{\circ} \mathrm{C}$ YMO (006) peak can be seen in Figure 33. Fits of the remaining samples can be found in Appendix A.4. 


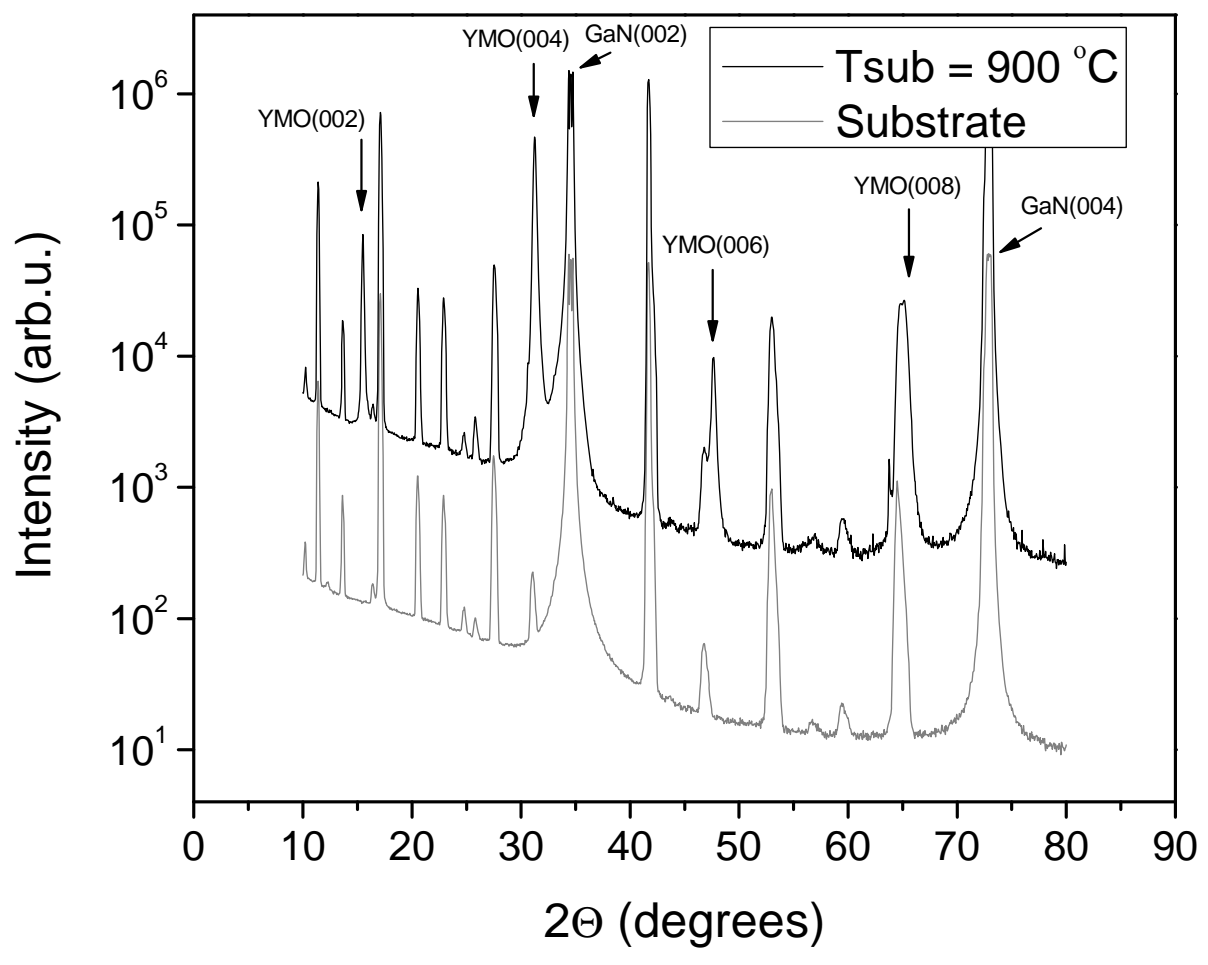

Figure 31: X-ray diffraction of sample grown at $900{ }^{\circ} \mathrm{C}$. Arrows indicate YMO $(00 l)$ reflections. All YMO $(00 l)$ reflections are strong. Unidentifiable peaks are located to low angle side of both the (004) and (008) reflections.
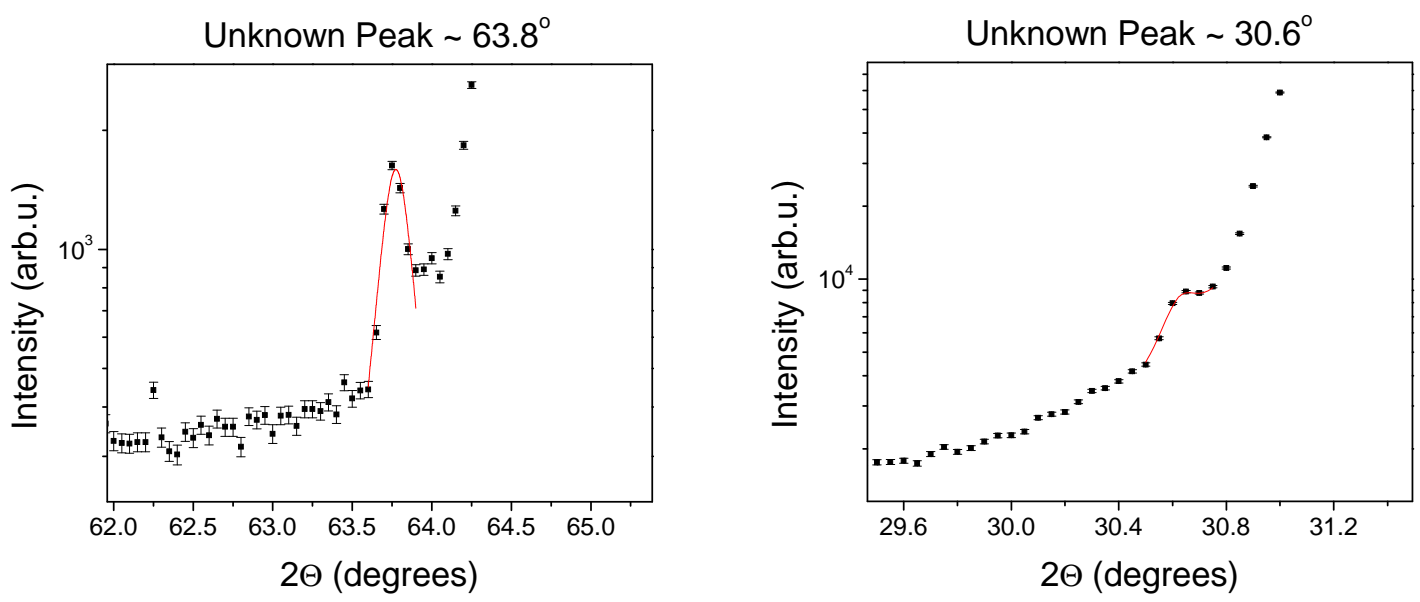

Figure 32: Unknown peaks located next to the YMO (008) and YMO (004) reflections, left to right. 


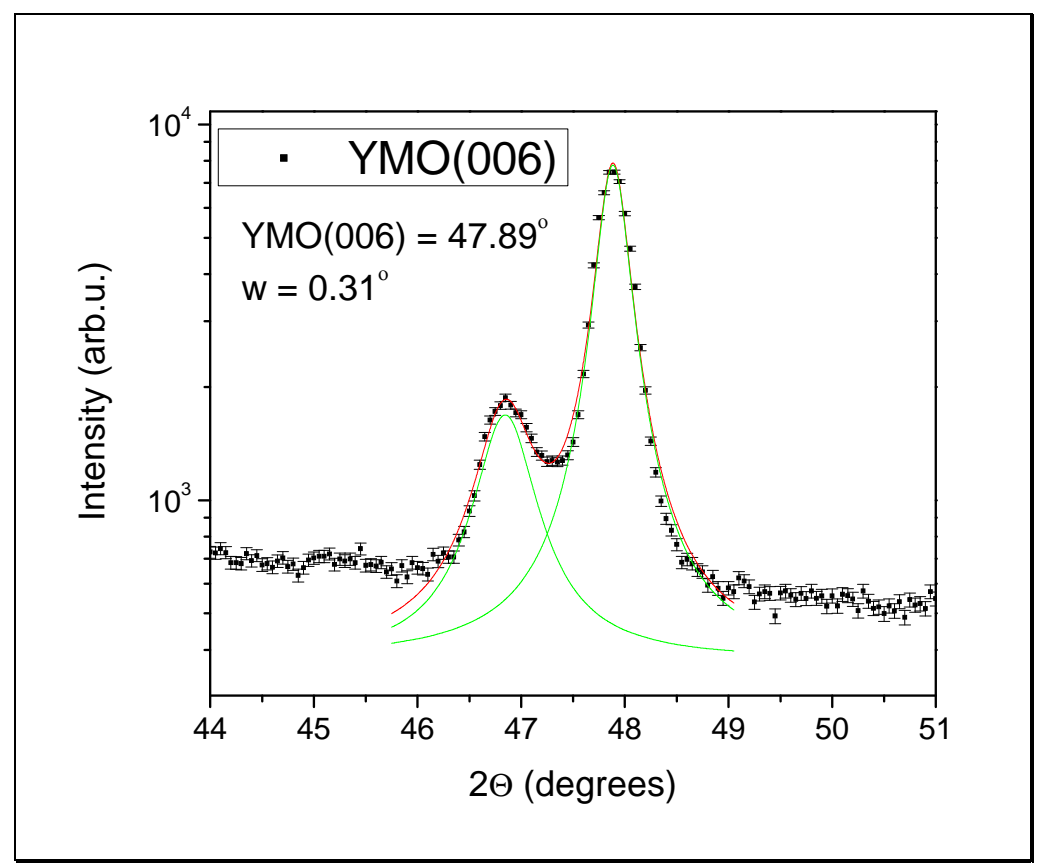

Figure 33: Lorentzian fit of the YMO (006) peak and nearby substrate peak. Scatter markers denote the measured data along with the associated error. Solid lines indicate the fit to each peak and the combined fit $\left(775^{\circ} \mathrm{C}\right)$.

Table 2: YMO (006) peak centroid and calculated c-axis lattice constant versus growth temperature.

\begin{tabular}{|c|c|c|}
\hline Growth Temperature $\left({ }^{\circ} \mathrm{C}\right)$ & $\begin{array}{c}\text { Peak Centroid } \\
\text { (degrees) }\end{array}$ & $\begin{array}{c}\text { c-Axis }(\AA)+/- \\
.01\end{array}$ \\
\hline 775 & 47.64 & 11.45 \\
\hline 800 & 47.92 & 11.39 \\
\hline 825 & 47.64 & 11.45 \\
\hline 850 & 47.83 & 11.41 \\
\hline 875 & 47.65 & 11.45 \\
\hline 900 & 47.89 & 11.4 \\
\hline
\end{tabular}

A rocking curve of the $775{ }^{\circ} \mathrm{C}$ sample is shown in Figure 34 , where the circles indicate measured data and the line a lorentzian fit. Data and fits of the $800{ }^{\circ} \mathrm{C}$ through $900{ }^{\circ} \mathrm{C}$ samples are located in Appendix A.5. The rocking curves of the YMO (002) reflection of each sample were normalized to a detector on the x-ray 
diffraction system placed to record the x-ray beam intensity, each sample thickness, and the resulting highest intensity data point for any sample. In this way, as long the sample size is kept relatively constant and the sample morphologies do not vary too widely (i.e. the surface roughnesses), the intensity of the rocking curve, not only the width, will be an approximate indicator of the sample quality. This is a valid assumption when comparing the small roughness variation determined for the surfaces given by x-ray reflectivity (Figure 30). Figure 35 displays the rocking curves for the samples together for a direct comparison. It is clear that the samples grown at $875^{\circ} \mathrm{C}$ and $900{ }^{\circ} \mathrm{C}$ had the most intense and narrowest rocking curves. Following the Ewald construction, this indicates that these samples displayed the smallest width in reciprocal space and consequently the best crystallinity. The sample grown at $775{ }^{\circ} \mathrm{C}$ also had a narrow rocking curve, but the intensity of the reflection was comparable to the samples with wide features. Figure 36 displays the FWHM of each rocking curve. It is again evident that the samples grown at high temperature were the most crystalline. 


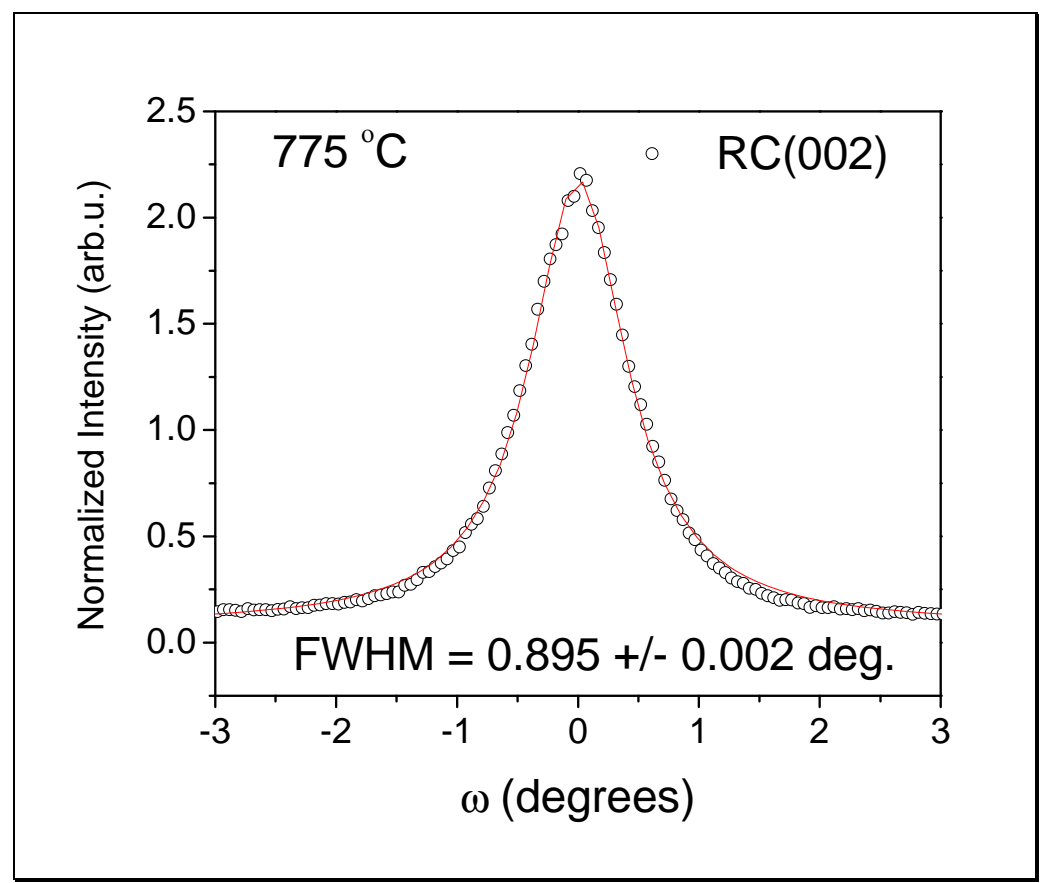

Figure 34: Rocking curve of $\mathrm{YMO}(002)$ reflection $\left(775^{\circ} \mathrm{C}\right)$. Circles indicate measured data and the solid line a lorentzian fit.

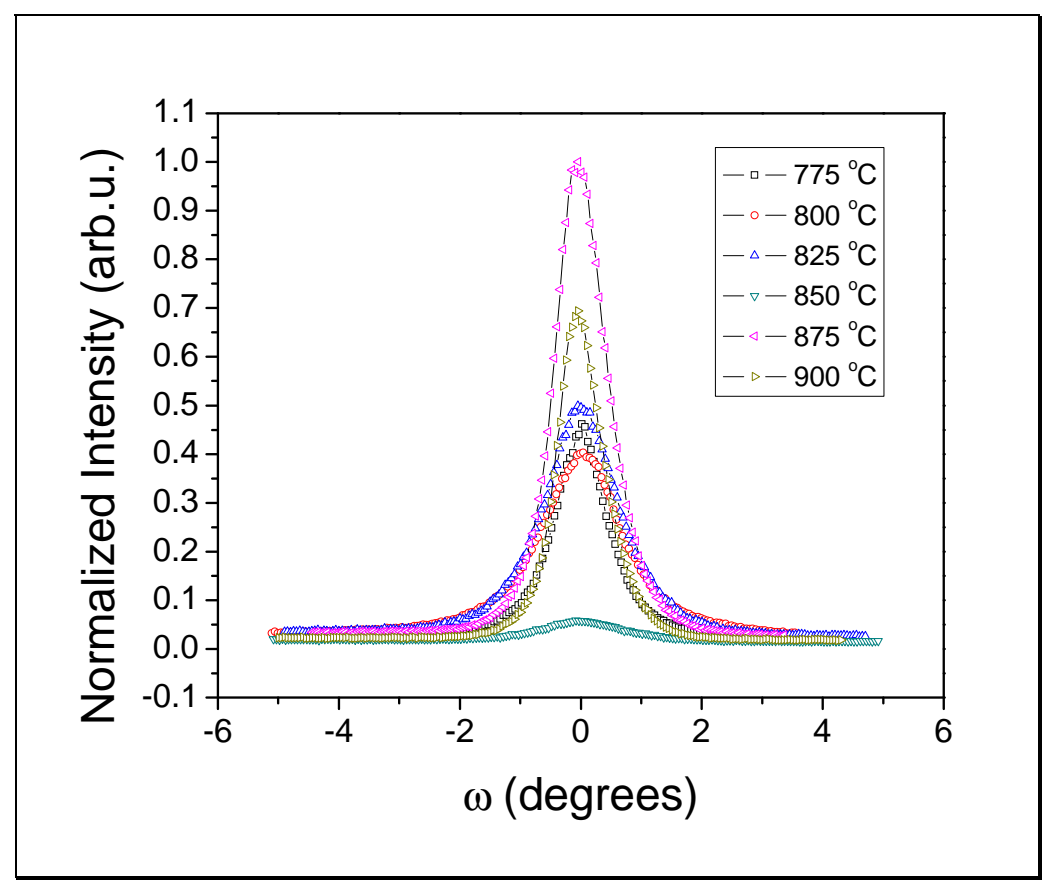

Figure 35: Comparison of the YMO (002) rocking curves for indicated growth temperatures. 


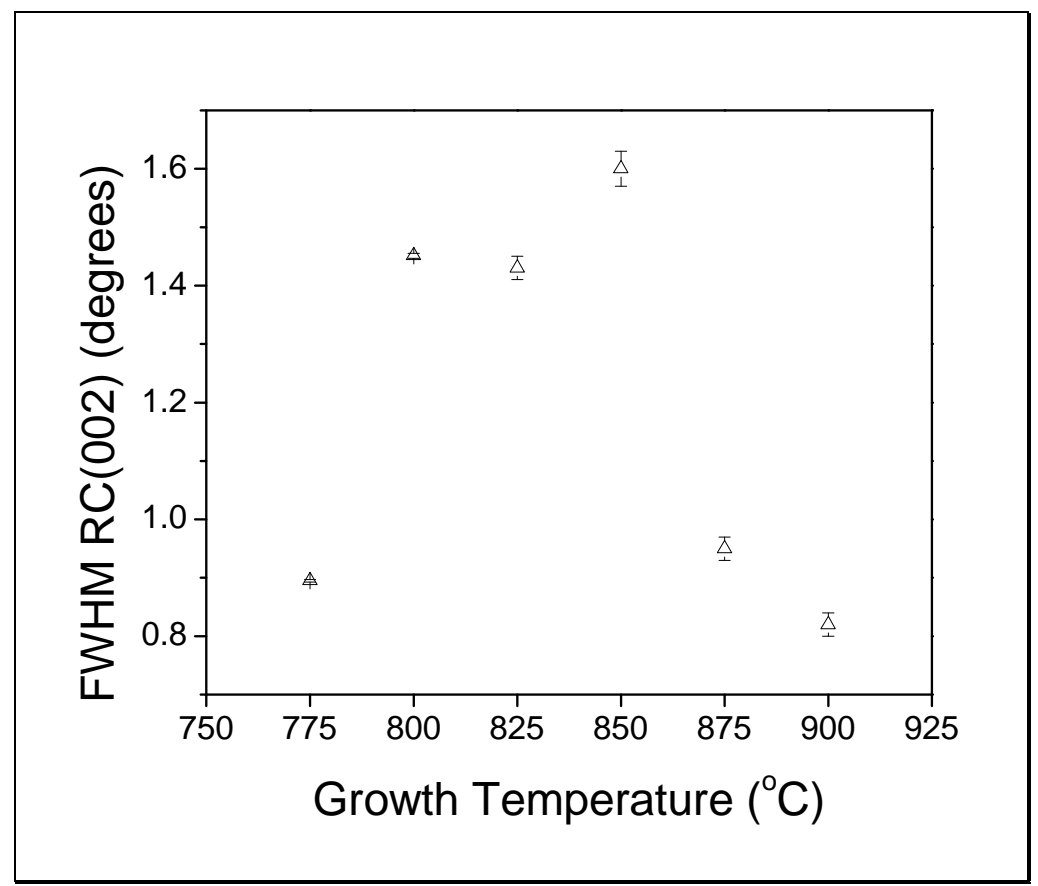

Figure 36: FWHM of the YMO (002) rocking curves as a function of growth temperature.

\subsubsection{In-Plane X-Ray Diffraction}

Figure 37 and Figure 38 are examples of in-plane diffraction scans from the $775{ }^{\circ} \mathrm{C}$ sample. In-plane x-ray diffraction results for other samples are summarized in Appendix A.6. All samples had the epitaxial relation YMO [1-10] $\| \mathrm{GaN}[110]$ determined by scanning the $\phi$ angle orientations of the YMO (106) or YMO (112) reflections and comparing with the $\phi$ angle orientations of the GaN (104) reflections. For clarification of which angle corresponds to $\phi$ refer to Figure 17. This epitaxial relation indicates that all samples had a $30^{\circ}$ rotation in the basal plane compared to the ideal lattice configuration. Using the angles obtained for the YMO (006) reflection and the YMO (106) or YMO (112) reflections, the a-axis lattice constant was determined for each sample. Table 3 is 
a summary of the a-axis lattice parameters versus growth temperature. No obvious trend can be observed. Aside from the samples grown at $825^{\circ} \mathrm{C}$ and 850 ${ }^{\circ} \mathrm{C}$, the lattice constants were roughly constant, with a value of $6.13+/-.02 \AA$, the same as the value found in bulk powder samples.

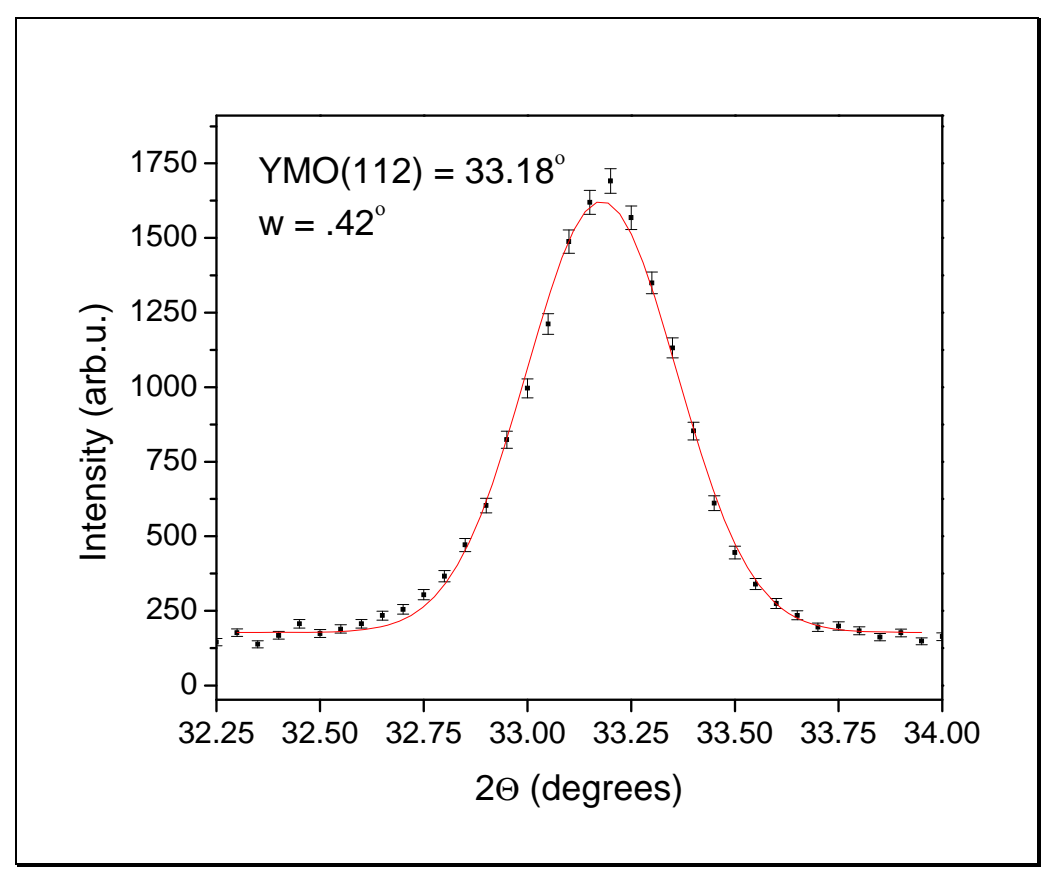

Figure 37: Gaussian fit of the YMO (112) reflection. Scatter markers denote the measured data along with the associated error. The solid line indicates the fit $\left(775^{\circ} \mathrm{C}\right)$. 


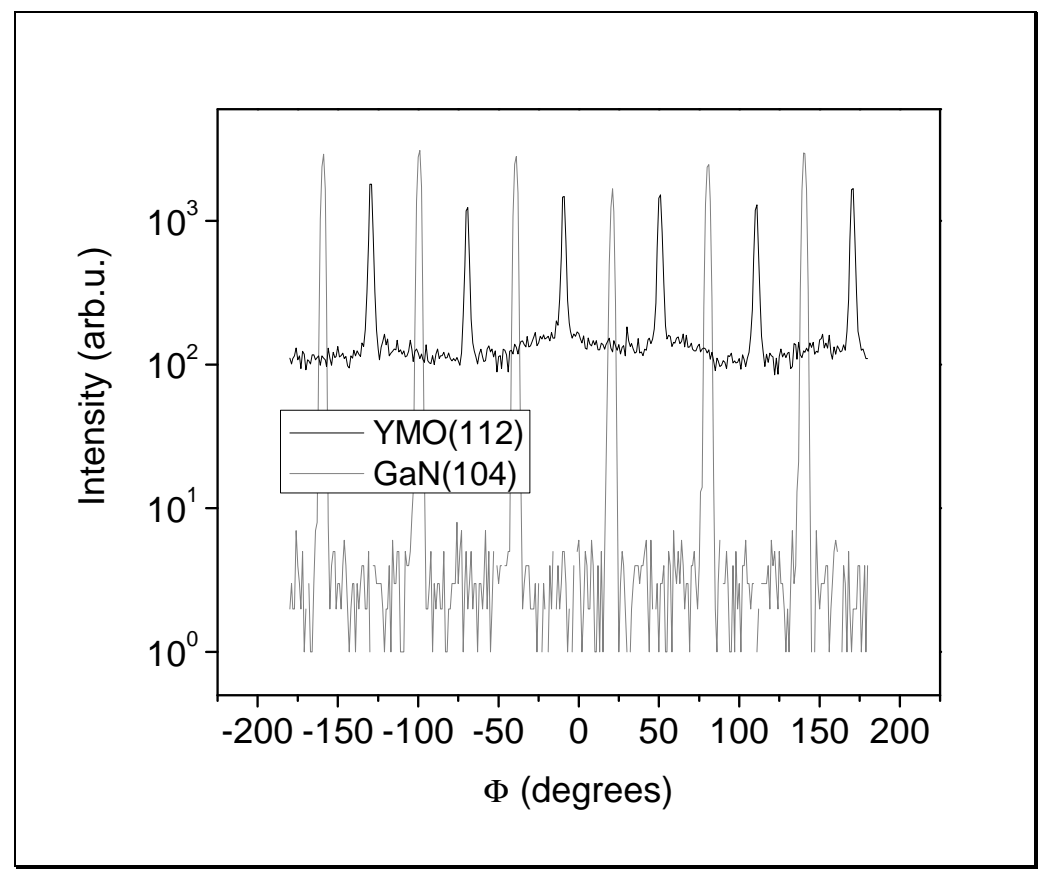

Figure 38: X-ray $\boldsymbol{\phi}$ scan indicating the in-plane epitaxial relation of YMO [1-10] || GaN [110] for the sample grown at $775^{\circ} \mathrm{C}$.

Table 3: a-axis lattice parameter versus growth temperature.

\begin{tabular}{|c|c|}
\hline Growth Temperature $\left({ }^{\circ} \mathrm{C}\right)$ & a-Axis $(\AA)+/-.01$ \\
\hline 775 & 6.14 \\
\hline 800 & 6.14 \\
\hline 825 & 6.25 \\
\hline 850 & 6.05 \\
\hline 875 & 6.12 \\
\hline 900 & 6.15 \\
\hline
\end{tabular}

\subsubsection{X-Ray Reflectivity}

Reflectivity data and fits for the samples grown at $775{ }^{\circ} \mathrm{C}$ and $900{ }^{\circ} \mathrm{C}$ are shown in Figure 39 and Figure 40, respectively. Data and fits for remaining samples are in Appendix A.7. Table 4 is a summary of the non-linear least squares fit to the x-ray reflectivity data for each sample. Error estimates for each parameter were determined by procedure where a single variable was modified 
until the value of $\chi^{2}$ changed by $5 \%$. Thickness estimates for the samples grown below $875{ }^{\circ} \mathrm{C}$ are all roughly equivalent, with the exception of the sample grown at $750{ }^{\circ} \mathrm{C}$. As the sample grown at $750{ }^{\circ} \mathrm{C}$ was not crystalline, this is not surprising. Both of the samples grown above $850{ }^{\circ} \mathrm{C}$ were deposited after the crystal was changed in the quartz crystal microbalance, most likely causing the change in deposition thickness.

One important trend revealed by the reflectivity analysis was the $\mathrm{GaO}$ thickness dependence as a function of growth temperature, illustrated in Figure 41. It is clear that during deposition the underlying GaN was decomposing and forming an oxide layer between the GaN substrate and the YMO. Comparing the reflectivity results from the samples grown at $775{ }^{\circ} \mathrm{C}$ and $900{ }^{\circ} \mathrm{C}$ (Figure 39 and Figure 40), one can see a drastic difference. As the deposition temperature increased, the $\mathrm{GaO}$ layer thickness became more apparent in the reflectivity as a modulation of slowly increasing frequency convoluted with the expected YMO reflectivity. On the other hand, as the GaN was decomposing, it did not increase in roughness significantly as a function of temperature. 


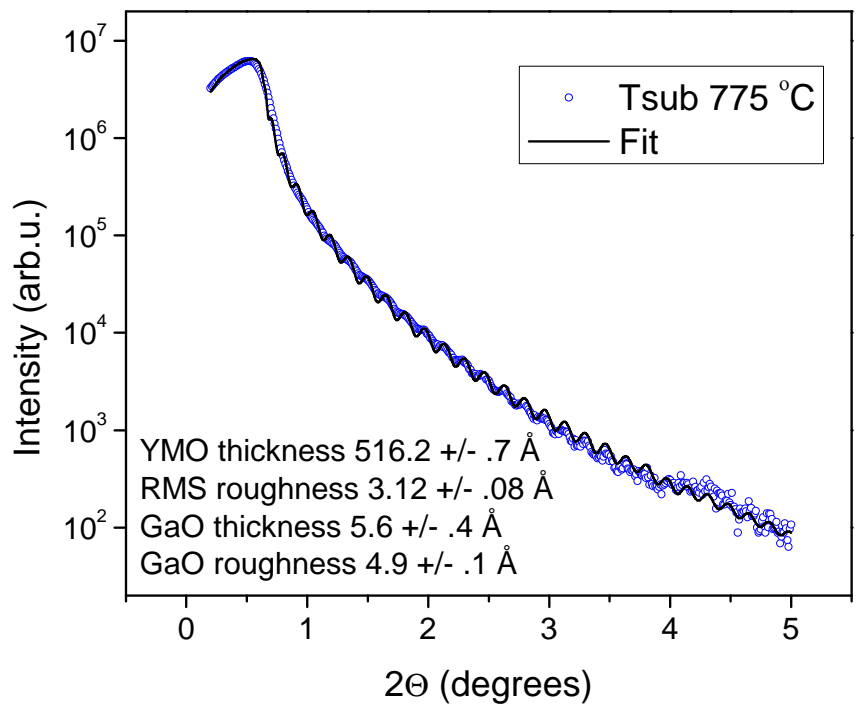

Figure 39: X-ray reflectivity of sample grown at $775^{\circ} \mathrm{C}$.

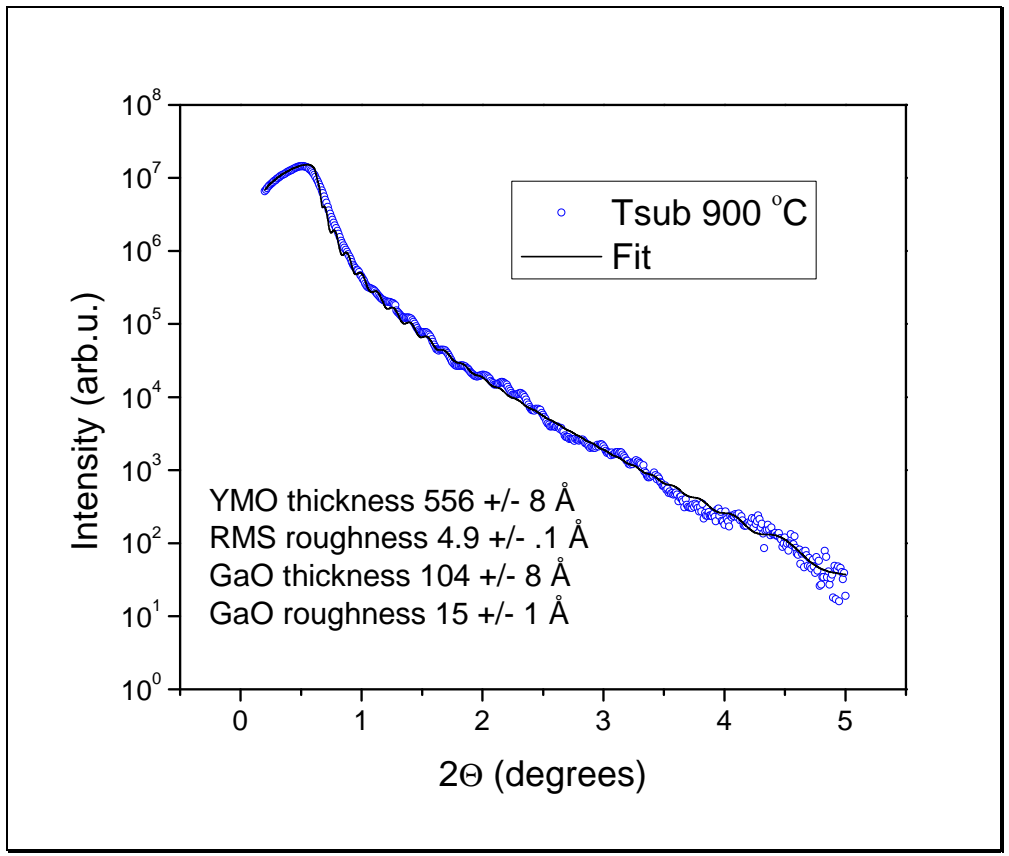

Figure 40: X-ray reflectivity of sample grown at $900{ }^{\circ} \mathrm{C}$. 
Table 4: X-ray reflectivity analysis summary.

\begin{tabular}{|c|c|c|c|c|c|c|c|}
\hline 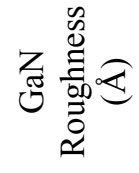 & $\begin{array}{l}m \\
? \\
i \\
+ \\
i \\
i n\end{array}$ & $\begin{array}{l}n \\
1 \\
+ \\
0 \\
0\end{array}$ & $\begin{array}{l}m \\
\stackrel{1}{1} \\
+ \\
\pm\end{array}$ & $\begin{array}{l}\overrightarrow{1} \\
\frac{1}{+} \\
=\end{array}$ & $\begin{array}{l}7 \\
\frac{1}{+} \\
0 \\
0\end{array}$ & $\begin{array}{l}n \\
\frac{1}{f} \\
6\end{array}$ & $\begin{array}{l}\infty \\
\frac{1}{+} \\
m \\
-\end{array}$ \\
\hline 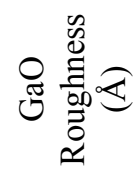 & 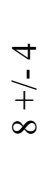 & $\begin{array}{l}\vec{r} \\
\dot{1} \\
\dot{+} \\
\dot{+}\end{array}$ & $\begin{array}{c}\stackrel{1}{1} \\
\frac{1}{+} \\
\end{array}$ & $\begin{array}{l}0 \\
i \\
\dot{+} \\
\infty \\
\dot{+}\end{array}$ & $\begin{array}{l}m \\
\frac{1}{+} \\
m \\
m\end{array}$ & $\begin{array}{l}0 \\
\frac{1}{1} \\
+ \\
0 \\
0\end{array}$ & $\begin{array}{l}\frac{7}{1} \\
+ \\
n\end{array}$ \\
\hline 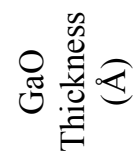 & $\begin{array}{l}m \\
1 \\
\frac{1}{+} \\
\infty \\
\infty\end{array}$ & 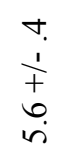 & $\begin{array}{l}\frac{4}{9} \\
\frac{1}{1} \\
\frac{1}{4}\end{array}$ & $\begin{array}{l}m \\
1 \\
+ \\
n \\
n\end{array}$ & $\begin{array}{l}\frac{r}{1} \\
\frac{1}{+} \\
m\end{array}$ & $\begin{array}{l}\frac{1}{9} \\
\frac{1}{1} \\
\frac{1}{2}\end{array}$ & $\begin{array}{l}\infty \\
\stackrel{1}{+} \\
\dot{+} \\
\dot{0}\end{array}$ \\
\hline 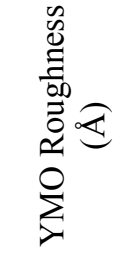 & 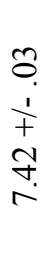 & $\begin{array}{l}\infty \\
0 \\
i \\
+ \\
\stackrel{1}{+} \\
\stackrel{m}{m}\end{array}$ & $\begin{array}{l}\overrightarrow{7} \\
\frac{1}{ \pm} \\
\overrightarrow{+}\end{array}$ & $\begin{array}{l}\vec{i} \\
\overrightarrow{+} \\
\vec{n}\end{array}$ & 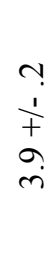 & $\begin{array}{l}\stackrel{+}{1} \\
\dot{+} \\
\stackrel{+}{+}\end{array}$ & $\begin{array}{l}\overrightarrow{7} \\
\dot{1} \\
\dot{+} \\
\dot{f}\end{array}$ \\
\hline 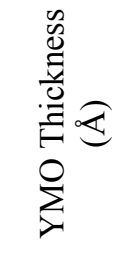 & $\begin{array}{l}\infty \\
\frac{1}{1} \\
\frac{n}{n}\end{array}$ & 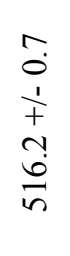 & $\begin{array}{l}1 \\
1 \\
+ \\
\text { In } \\
n\end{array}$ & $\begin{array}{l}\dot{0} \\
i \\
\dot{+} \\
0 \\
\stackrel{a}{n}\end{array}$ & $\begin{array}{l}n \\
\frac{1}{+} \\
\frac{1}{n}\end{array}$ & $\begin{array}{l}8 \\
\text { 1 } \\
1 \\
+ \\
0 \\
i n \\
n\end{array}$ & $\begin{array}{l}\infty \\
1 \\
+ \\
0 \\
0 \\
n\end{array}$ \\
\hline 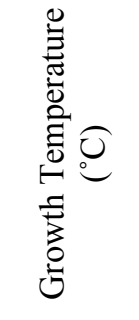 & $\stackrel{2}{n}$ & $\stackrel{n}{\curvearrowright}$ & \& & స్ & ஜे & $\stackrel{n}{\infty}$ & ৪ু \\
\hline
\end{tabular}




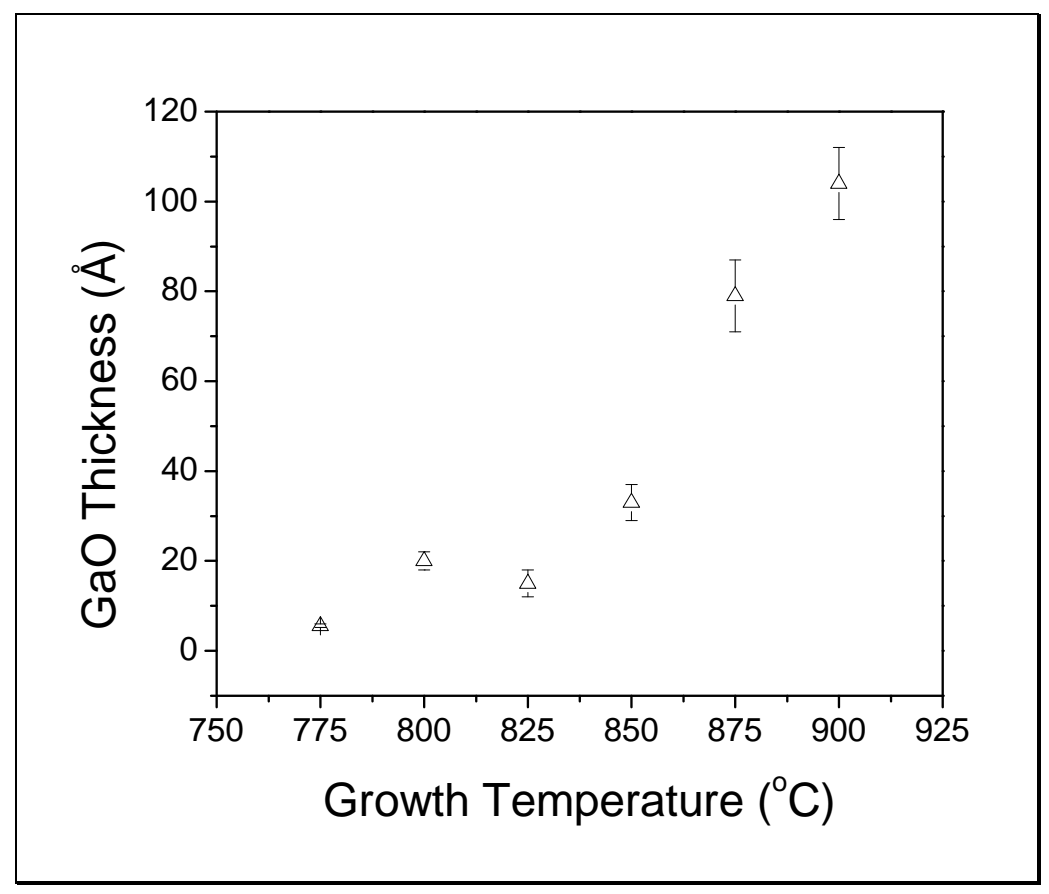

Figure 41: $\mathrm{GaO}$ thickness versus sample growth temperature.

\subsection{Electrical Characterization Results}

$P-V$ measurements performed on the samples grown from $775{ }^{\circ} \mathrm{C}$ to $900{ }^{\circ} \mathrm{C}$ showed very small remanence and no sign of saturation. Measurements were performed with a period of $10 \mathrm{~ms}$ in order to minimize the amount of leakage current that would be integrated during each measurement cycle. Unfortunately, the number of data points acquired by the tester was limited by the period of the measurement cycle. So, for the samples investigated, smaller measurement periods could not be used, due to the small size of the measured response and the noise level of the system. Figure 42 includes two hysteresis loops taken using .5V (left panel) and $1 \mathrm{~V}$ (right panel). The measured remanence for the $.5 \mathrm{~V}$ test was approximately $5 \times 10^{-2} \mu \mathrm{C} / \mathrm{cm}^{2}$. This value is two orders of magnitude smaller than the value $3.2 \mu \mathrm{C} / \mathrm{cm}^{2}$, previously measured by Chye et.al. [31]. Comparing 
all hysteresis measurements performed (data located in Appendix A.8), lack of saturation and small remanence are common to all samples. $P-V$ measurements using a maximum voltage of $1 \mathrm{~V}$ show a distinct lack of loop closure, indicating large leakage currents during hysteresis measurement. Being more prominent in some samples than others, $I-V$ measurements were performed in order to determine what leakage mechanisms and whether there was any correlation between leakage and deposition temperature.
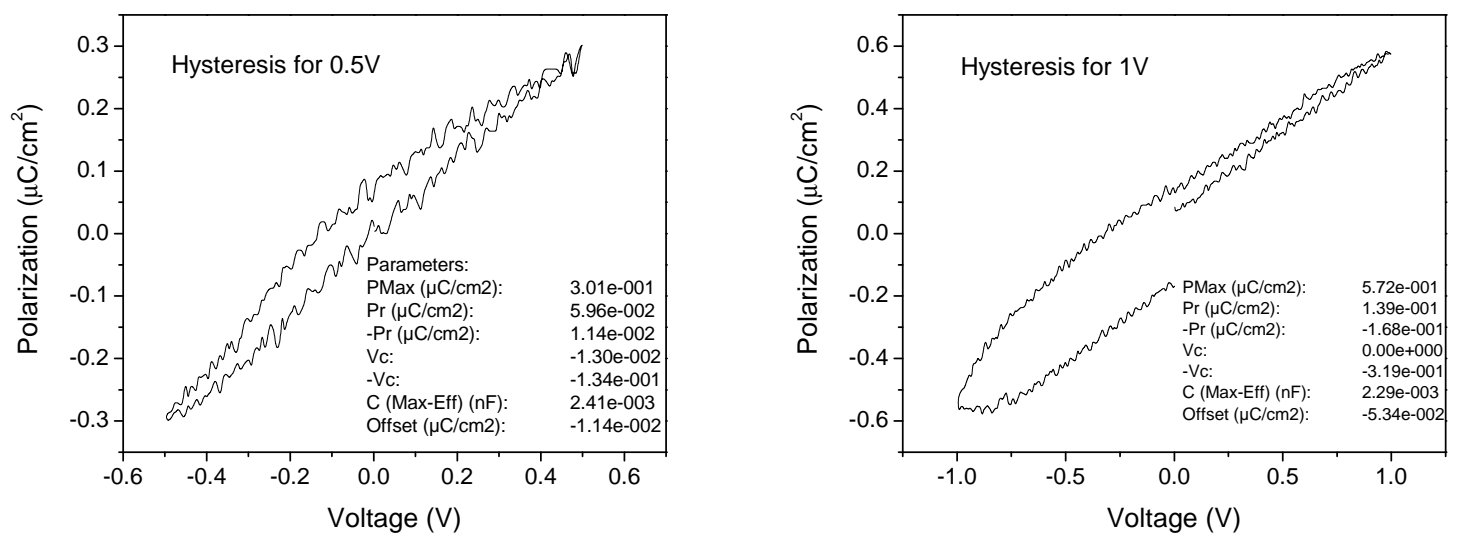

Figure 42: $P-V$ loops for $0.5 \mathrm{~V}$ and $1 \mathrm{~V}\left(775^{\circ} \mathrm{C}\right)$.

Figure 43 is the current density vs. electric field $(J-E)$ plot for the sample grown at $775{ }^{\circ} \mathrm{C}$. $I$ and $V$ were converted to $J$ and $E$ simply by dividing the measured current by the contact area and dividing the applied voltage by the sample thickness, respectively. The two most salient features of these data are the large size of $J$ and the asymmetry of the current density based on the voltage polarity. Note that the voltage was applied to the GaN contact and referenced from the grounded top Pt contact. It is clear from the plot that there are multiple 
leakage regimes or effects taking place during the measurement. Table 5 lists some of the most common conduction processes through insulators.

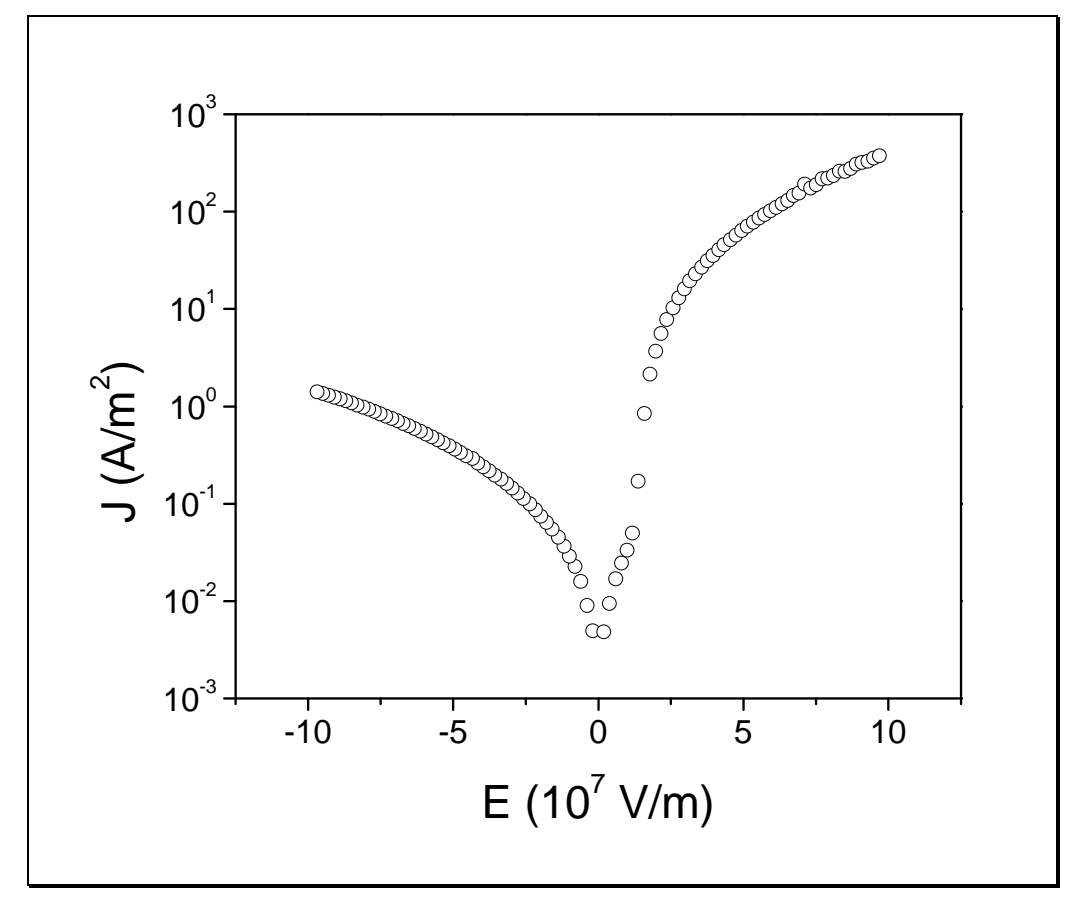

Figure 43: $\mathrm{J}$ vs. $\mathrm{E}$ for $775{ }^{\circ} \mathrm{C}$

At low fields the most common conduction mechanism is ohmic conduction. For ohmic conduction, the slope of $\log _{10} J$ versus $\log _{10} E$ is one. First examining the negative field direction for the sample grown at $775{ }^{\circ} \mathrm{C}$ (Figure 44), ohmic conduction evidently occurs at low voltages followed by and a transition to space charge limited conduction (SCLC) mechanism. SCLC can be identified by the characteristic square dependence upon the field and results from charge injection into a lightly doped material where, in the vicinity of a junction between two materials, doping concentrations $\mathrm{n}$ and $\mathrm{p}$ could be smaller or larger than the donor $\left(N_{D}\right)$ and acceptor $\left(N_{A}\right)$ concentrations, respectively. Typically, the region would be neutral, i.e. $n=N_{D}$ and $p=N_{A}$. Using the depletion approximation, where $\mathrm{n}$ and $\mathrm{p}$ are assumed to be zero, the space charge is then equal to the majority carrier 
doping level. Under the application of a field carriers can then be injected that are larger than the equilibrium values of the majority carrier doping level. This sets up an internal field that is controlled by the injected carrier profile. For electrons, the current due to drift effects follows

$$
J=q n v .
$$

Poisson's equation gives

$$
\frac{d^{2} \Psi}{d x^{2}}=\frac{q n}{\varepsilon_{s}}
$$

Then, assuming a low mobility regime, where $v=\mu E$, the expression for the current can be solved with the result

$$
J=\frac{9 \varepsilon_{s} \mu V^{2}}{8 d^{3}} .
$$

The transition from ohmic conduction to SCLC takes place at a field of approximately $2 \times 10^{7} \mathrm{~V} / \mathrm{m}$ in the $775{ }^{\circ} \mathrm{C}$ samples. This transition point is roughly the same for the sample grown at $800{ }^{\circ} \mathrm{C}$. Above this temperature the samples do not display a transition and show ohmic conduction across the entire measured range. Data and fits for samples other than the growth at $775{ }^{\circ} \mathrm{C}$ are located in Appendix A.8. 


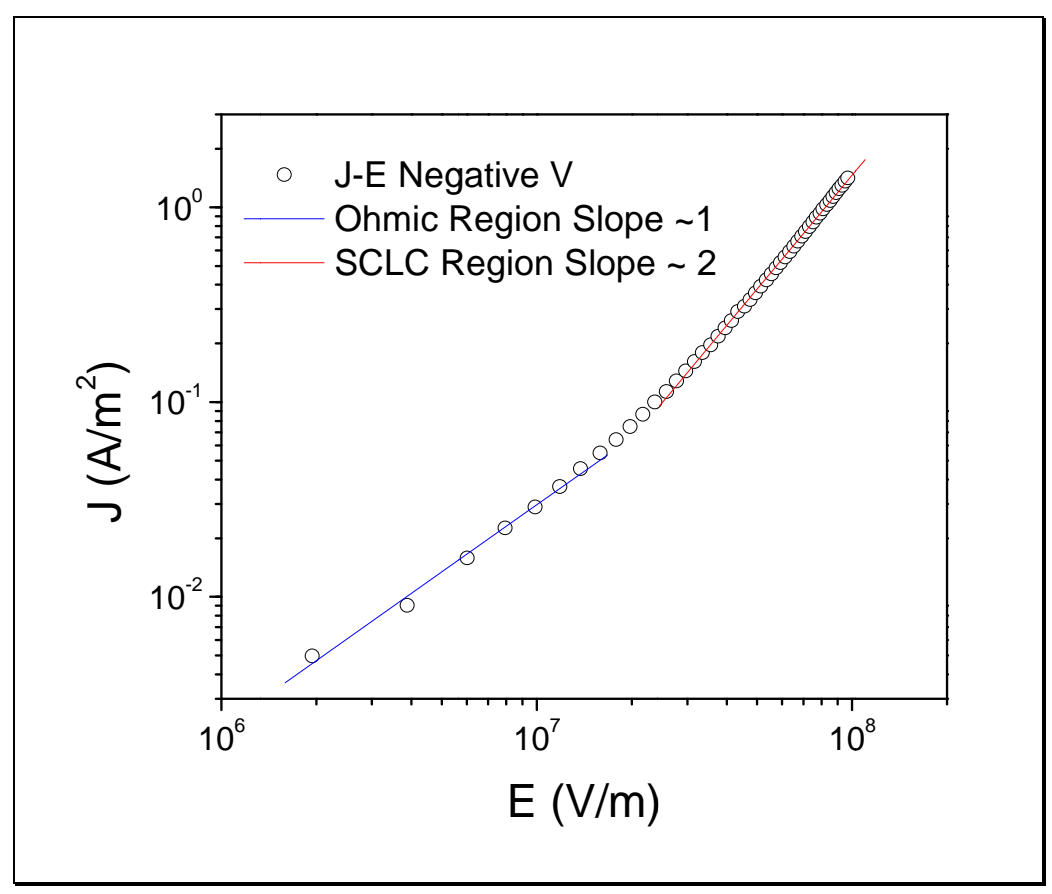

Figure 44: $\log \mathrm{J}$ vs. $\log \mathrm{E}$ for negative voltage $\left(775^{\circ} \mathrm{C}\right)$. Each solid, colored line represents a linear fit to a separate leakage region.

Table 5: Basic Conduction Processes in Insulators [59].

\begin{tabular}{|c|c|c|}
\hline Process & Expression & $\begin{array}{l}\text { Voltage \& temperature } \\
\text { dependence }\end{array}$ \\
\hline Tunneling & $J \propto \mathscr{E}_{i}^{2} \exp \left[-\frac{4 \sqrt{2 m^{*}}\left(q \phi_{B}\right)^{3 / 2}}{3 q \hbar \mathscr{C}_{i}}\right]$ & $\propto V^{2} \exp \left(\frac{-b}{V}\right)$ \\
\hline $\begin{array}{c}\text { Thermionic } \\
\text { emission }\end{array}$ & $J=A^{* *} T^{2} \exp \left[\frac{-q\left(\phi_{B}-\sqrt{q \mathscr{E}_{i} / 4 \pi \varepsilon_{i}}\right)}{k T}\right]$ & $\propto T^{2} \exp \left[\frac{q}{k T}\left(a \sqrt{V}-\phi_{B}\right)\right]$ \\
\hline $\begin{array}{l}\text { Frenkel-Poole } \\
\text { emission }\end{array}$ & $J \propto \mathscr{E}_{i} \exp \left[\frac{-q\left(\phi_{B}-\sqrt{q \mathscr{E}_{i} / \pi \varepsilon_{i}}\right)}{k T}\right]$ & $\propto V \exp \left[\frac{q}{k T}\left(2 a \sqrt{V}-\phi_{B}\right)\right.$ \\
\hline Ohmic & $J \propto \mathscr{E}_{i} \exp \left(\frac{-\Delta E_{a c}}{k T}\right)$ & $\propto V \exp \left(\frac{-c}{T}\right)$ \\
\hline Ionic conduction & $J \propto \frac{\mathscr{E}_{i}}{T} \exp \left(\frac{-\Delta E_{a i}}{k T}\right)$ & $\propto \frac{V}{T} \exp \left(\frac{-d}{T}\right)$ \\
\hline $\begin{array}{l}\text { Space-charge- } \\
\text { limited }\end{array}$ & $J=\frac{9 \varepsilon_{i} \mu V^{2}}{8 d^{3}}$ & $\propto V^{2}$ \\
\hline
\end{tabular}

$A^{* *}=$ effective Richardson constant. $\phi_{B}=$ barrier height. $\mathscr{E}_{i}=$ electric field in insulator. $\varepsilon_{i}=$ insulator permittivity. $m^{*}=$ effective mass. $d=$ insulator thickens. $\Delta E_{a c}=$ activation energy of electrons. $\Delta E_{a i}=$ activation energy of ions, $V \approx \mathscr{E}, d, a \equiv \sqrt{q / 4 \pi \varepsilon_{i} d} . b, c$, and $d$ are constants. 
Examination of the positive voltage direction also showed ohmic and SCLC conduction along with an eventual transition to Schottky emission at high fields. Figure 45 shows positive voltage $\log J$ versus $\log E$ plot with associated fits for the $775{ }^{\circ} \mathrm{C}$ sample. SCLC conduction under the positive field direction was followed by a behavior indicative of an exponential trap distribution,

$$
J=N_{c} \mu q^{(l-1)}\left[\frac{e l}{N_{t}(l+1)}\right]\left(\frac{2 l+1}{l+1}\right)^{(l+1)} \frac{V^{(l+1)}}{d^{(2 l+1)}},
$$

where $l=T_{c} / T, N_{c}$ is the effective density of states in the conduction band and $N_{t}$ is the trap density [60]. This behavior is an indication of a transition from SCLC with trap limited conduction to trap free conduction. $l$ for samples $775{ }^{\circ} \mathrm{C}$ to $875{ }^{\circ} \mathrm{C}$ ranged from 4 to 11 , giving a value of the constant $T_{c}$ a spread from $1200 \mathrm{~K}$ to $3300 \mathrm{~K}$. The constant $T_{c}$ is associated with the distribution of traps through the expression

$$
N_{t}(E)=\frac{N_{t}}{k T_{c}} \exp \left[\frac{E-E_{c}}{k T_{c}}\right]
$$

where $N_{t}(E)$ is the trap concentration per unit energy range [60].

Above approximately $2 \times 10^{7} \mathrm{~V} / \mathrm{m}$ the conduction transitions to Schottky emission. Figure 46 shows the fit for the $775^{\circ} \mathrm{C}$ sample to the Schottky emission model. Estimations of $\varepsilon$, the relative permittivity, for samples $775^{\circ} \mathrm{C}$ through 875 ${ }^{\circ} \mathrm{C}$ ranged from 9 to 13 . The value determined by Huang et.al. was 15 [61], indicating that the values determined here are reasonable. Data and fits to samples other than the $775^{\circ} \mathrm{C}$ sample are located in Appendix A.8. 
One potential mechanism for the high current density displayed by the samples may be the band alignment between the YMO and GaN. As insulators are essentially wide band gap semiconductors, depending upon the conduction band alignment between the two materials, at some applied field a relatively small barrier may be overcome allowing for high current densities. Possible band alignments are illustrated in Figure 47. Type I structures would allow for a wide gap insulator to conduct if the conduction bands of the insulator and the semiconductor are closely matched [45]. Photoelectron spectroscopy could be used to determine the band offsets between YMO and GaN [62]. For a review of this technique see Ref. [63].

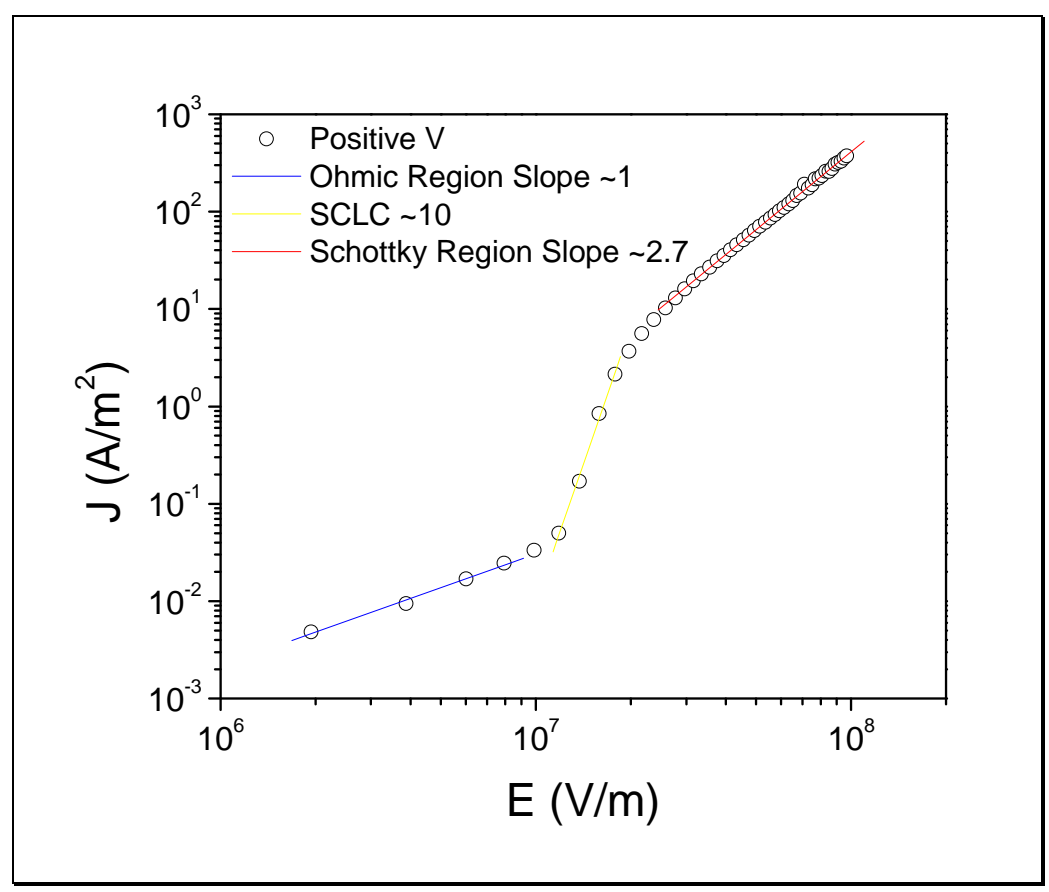

Figure 45: $\log J$ vs. $\log E$ for positive voltage $\left(775^{\circ} \mathrm{C}\right)$. Each solid, colored line represents a linear fit to a separate leakage region. 


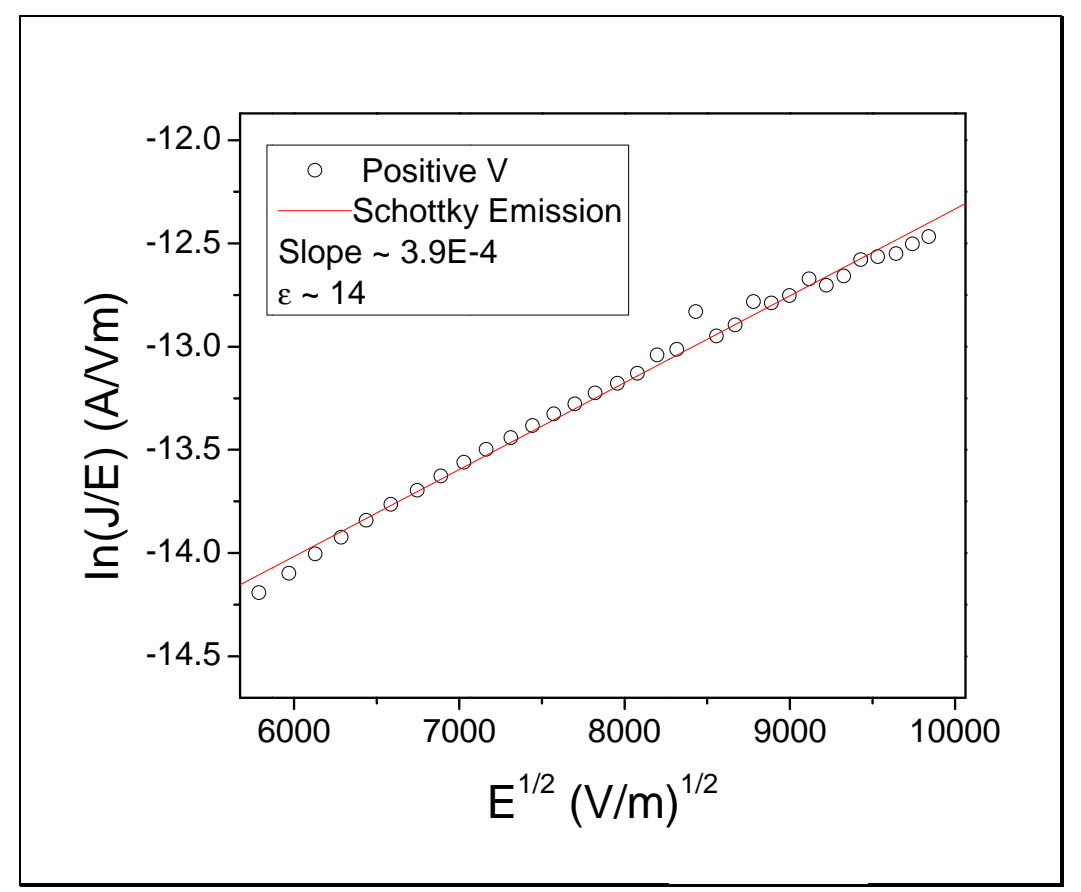

Figure 46: $\operatorname{Ln}(J / E)$ vs. $\mathrm{E}^{1 / 2}$ for positive voltage $\left(775^{\circ} \mathrm{C}\right)$. The line is the linear fit to region corresponding to Schottky emission.

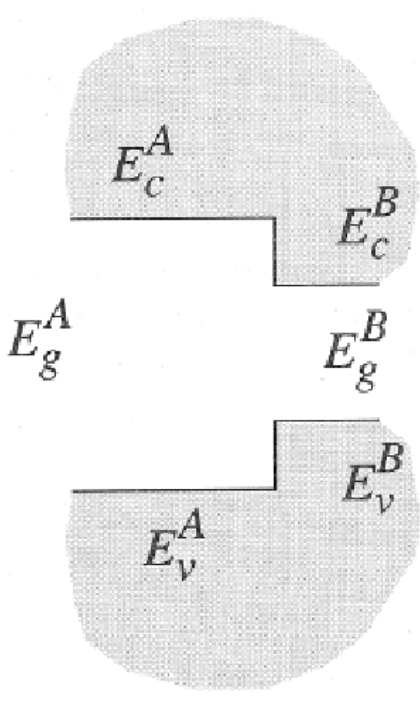

TYPE I

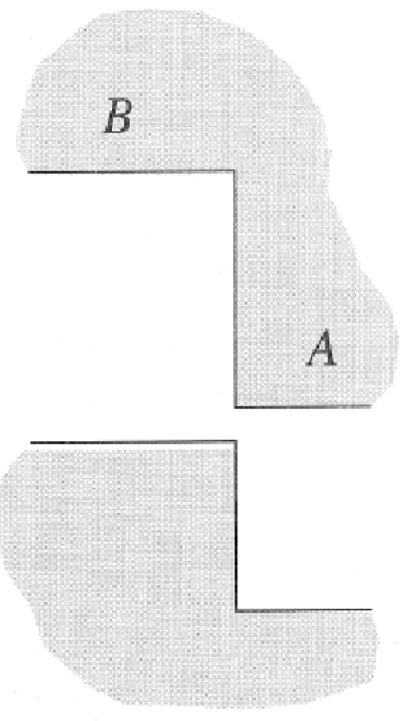

TYPE II

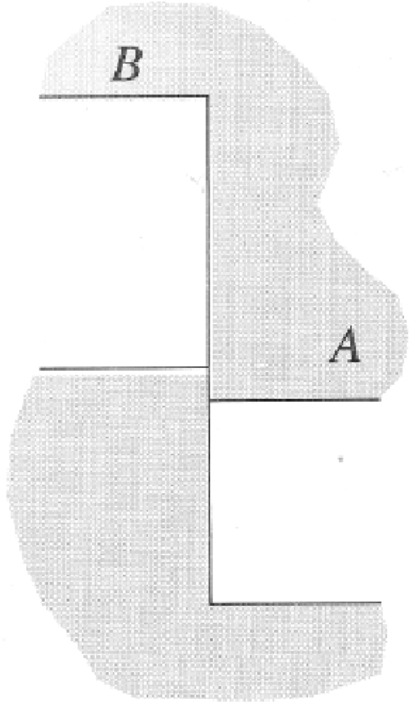

BROKEN GROUP

TYPE III

Figure 47: Various band edge alignments possible in heterostructures [45]. 
The sample grown at $900{ }^{\circ} \mathrm{C}$ stands out. It is the only sample not to display SCLC, illustrated in Figure 48. The most likely leakage mechanism in this case seems to be Fowler-Nordheim tunneling. Fowler-Nordheim tunneling is much the same as direct tunneling but defects lower the tunneling barrier, making it easier for tunneling to take place. Figure 49 shows the linear fit for the $900{ }^{\circ} \mathrm{C}$ sample to the Fowler-Nordheim model.

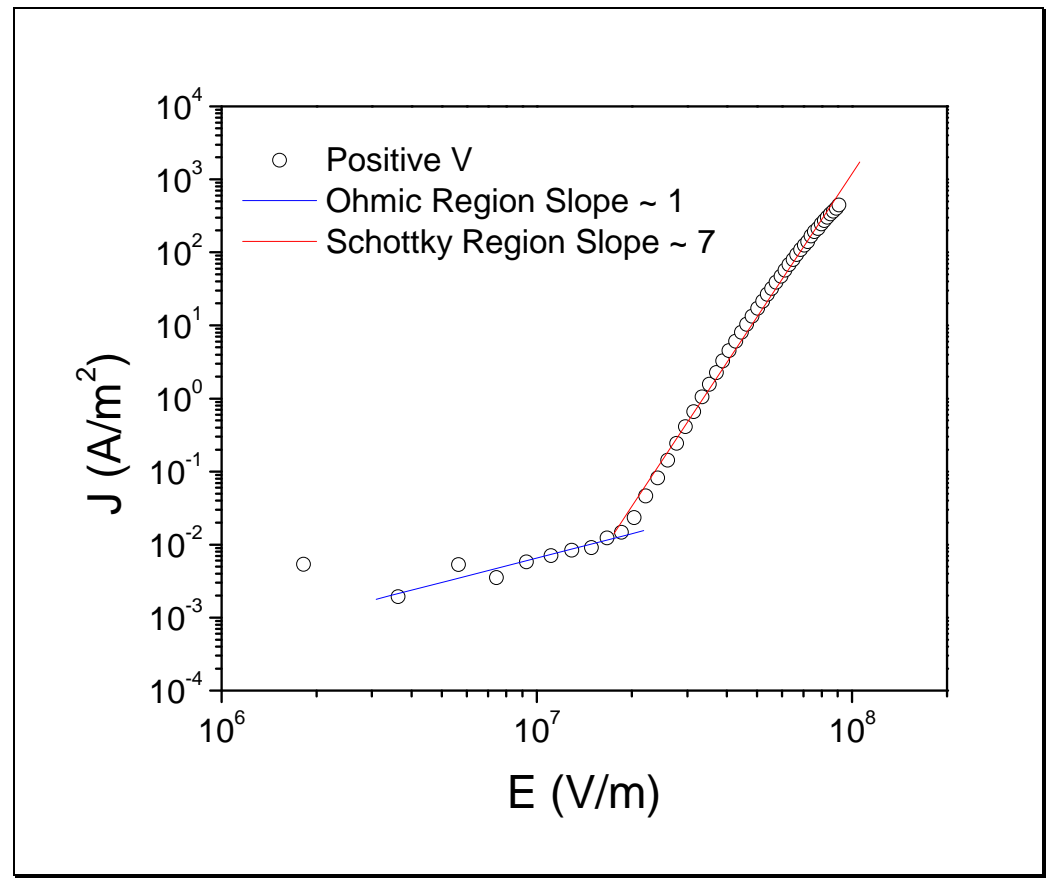

Figure 48: $\log J$ vs. $\log E$ for positive voltage $\left(900{ }^{\circ} \mathrm{C}\right)$. Each solid, colored line represents a linear fit to a separate leakage region. 


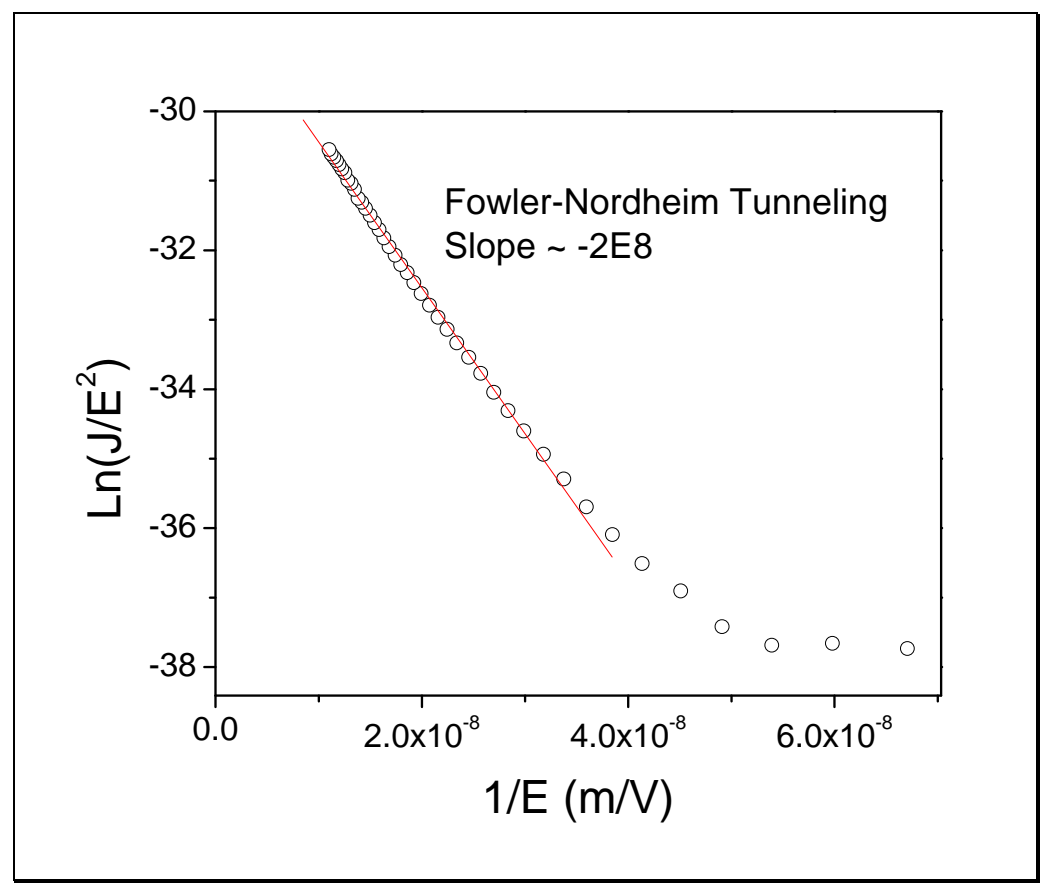

Figure 49: Fowler-Nordheim Tunneling model fit to sample grown at $900{ }^{\circ} \mathrm{C}$. 


\section{Chapter 5: Scanning Surface Potential Microscopy}

As mentioned previously, samples grown using alumina sample holders suffered from poor thermal contact and indium contamination. This contamination resulted in large prism formations that were initially believed to consist of YMO. It was later revealed via electron energy loss spectroscopy (EELS) that these features did not contain Mn, but did contain In. This contamination was not seen in samples grown on Si wafers.

During deposition the first indication of an anomalous surface consisted of noticeable transmission spots in the RHEED that became visible after the deposition of roughly 5 unit cells worth of YMO, illustrated in Figure 50(a). As this was not the desired surface structure, once it was evident that the surface was growing in a three-dimensional fashion growth was stopped. The sample(s) were subsequently removed from the chamber and analyzed in order to determine the mechanism by which these formations were taking place.

AFM revealed these features to be large uniform prism formations with a regular orientation with respect to the substrate Figure 50(b). Heights of the formations varied from over a large range, with some of them being as large as roughly $60.0 \mathrm{~nm}$.

X-ray diffraction revealed two phases, shown in Figure 51, corresponding to lattice parameters $11.70 \pm 0.01 \AA$ and $11.68 \pm 0.01 \AA$, assuming both peaks correspond to $\mathrm{YMO}(008)$ reflections. 
As it was initially believed that these features consisted of YMO, it was proposed that these features may have enhanced polarization compared to thin films, as there would be less in plane clamping from the surrounding material. As this made the prisms promising for self-assemble device contacts, a method was needed in order to determine if the structures were ferroelectric. Scanning surface potential microscopy was implemented for this specific task.

Scanning surface potential microscopy (SSPM) is an interleave AFM technique in which a surface is initially scanned for topography and then subsequently rescanned with the tip held a fixed distance above the surface, much like magnetic force microscopy [64]. In this particular mode of operation, information can be obtained that does not contain topographic information. Figure 52 illustrates the principle of operation of SSPM. In order to obtain potential mapping of the surface, SSPM uses a feedback loop where a lock-in amplifier measures the amplitude of the free oscillation of the cantilever as a combination of $\mathrm{AC}$ and $\mathrm{DC}$ voltages are applied to the tip. Simplifying the system as a parallel plate capacitor, Figure 53 illustrates the forces between the cantilever and the sample. With the lock-in amplifier monitoring the first harmonic of the cantilever oscillation, adjustment of the DC cantilever bias allowed for a direct mapping of the sample surface potential.

Poling the surface with different potentials between the sample and tip was used to establish whether or not the sample showed any ferroelectric response. This was performed for a series of prisms of varying size and for areas roughly $50.0 \mathrm{~nm}$ away from each prism. After poling, the surface areas were scanned with 
a lift height of $20.0 \mathrm{~nm}$ and $2 \mathrm{~V}$ AC bias. Prisms with different surface areas and heights were compared. Figure 54 (a) and (b) show the measured response for the prism with height $44 \mathrm{~nm}$ as a function of poling voltage. It is clear that the film had a weaker response than the prism to the poling voltage. As a method to quantify the effect of the surface poling, the remanent net potential was defined as $V_{R}=\left(V_{S^{+}}-V_{S_{-}}\right) / 2$ where $V_{S^{+}}$and $V_{S_{-}}$are the upper and lower branch responses at $V_{P}=0$, respectively. Figure 55 displays the dependence of the remanent net potential upon sample prism surface area. The data point corresponding to zero prism area was determined by averaging all of the potential measurements taken away from each prism. It was revealed that prisms with comparable surface area but varying heights had roughly constant remanent net potentials. The remanent net potential did, however, have a clear dependence upon prism surface area.

EELS performed on this particular sample demonstrated that the prisms did not contain Mn, as previously believed. The constituent materials turned out to be $\mathrm{Y}$, In, and $\mathrm{O} . \mathrm{YInO}_{3}$ is a material that can grow in either cubic or hexagonal phases (cubic lattice parameter $\mathrm{a}=10.36 \AA$, hexagonal lattice parameters $\mathrm{a}=6.25$ $\AA$ and $\mathrm{c}=12.25 \AA$ ). It appears from TEM and EELS that the prisms were crystalline structures composed of $\mathrm{YInO}_{3}$ which grow epitaxially on a very thin layer of $\mathrm{YMnO}_{3}$. Little research has been done on $\mathrm{YInO}_{3}$, particularly in thin film form. So, this result is fortuitous. It may lead to research into a new material for ferroelectric device structures. 


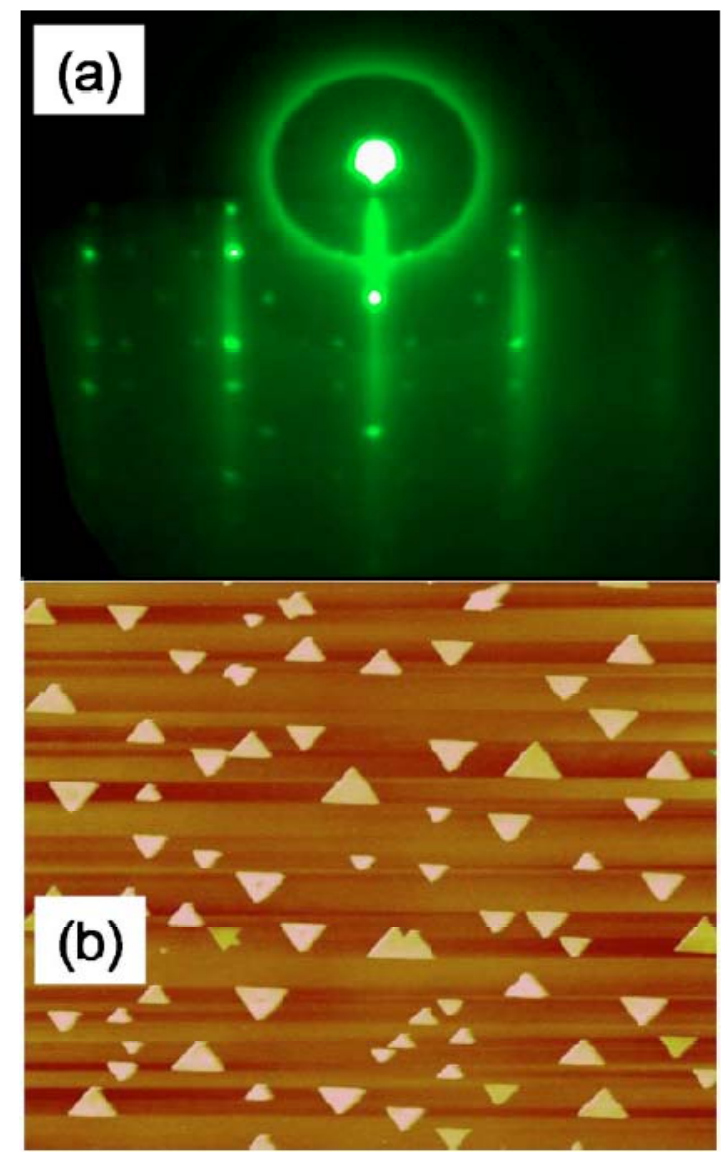

Figure 50: (a) RHEED image obtained with the electron beam along the YMO [110] direction after six unit cells of growth on GaN. (b) AFM image $\left(10 \times 10 \mu \mathrm{m}^{2}\right)$ of the same sample showing the prism structures [65]. 


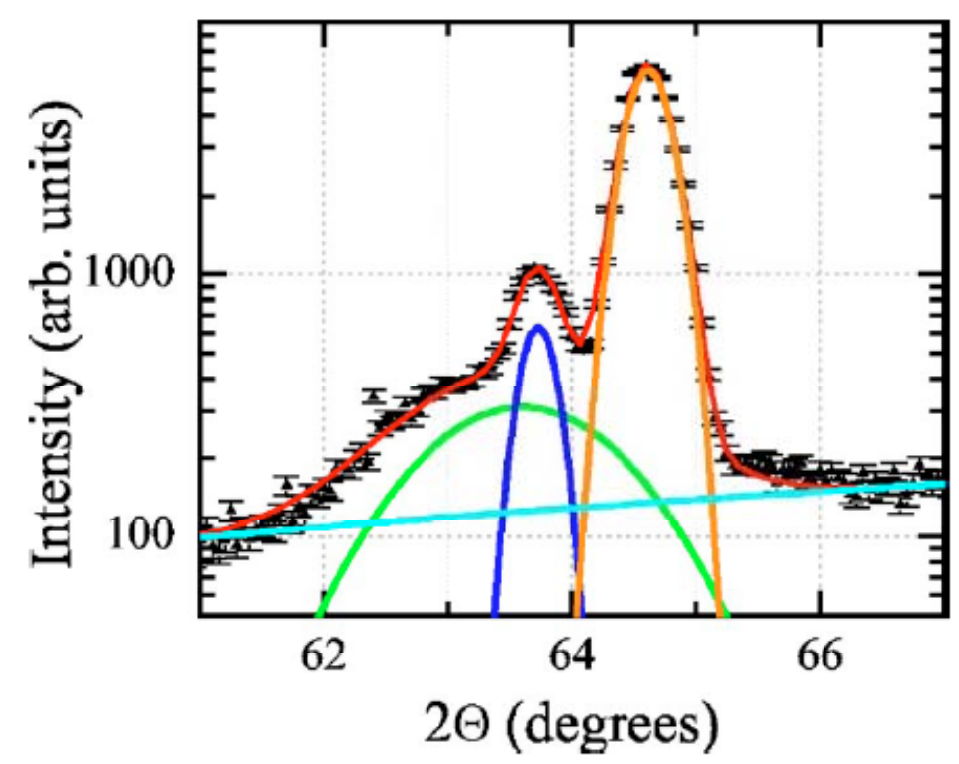

Figure 51: High angle $\omega-2 \theta$ scan obtained for the sample in Figure 50. The green, blue, and orange curves are fits of three Gaussians to the spectrum corresponding the film, prism, and substrate $\left(\mathrm{Al}_{2} \mathrm{O} 3\right)$ peaks on a linear background (light blue line). The red line is the sum of all these contributions. The film and prism peaks correspond to the (008) reflections.

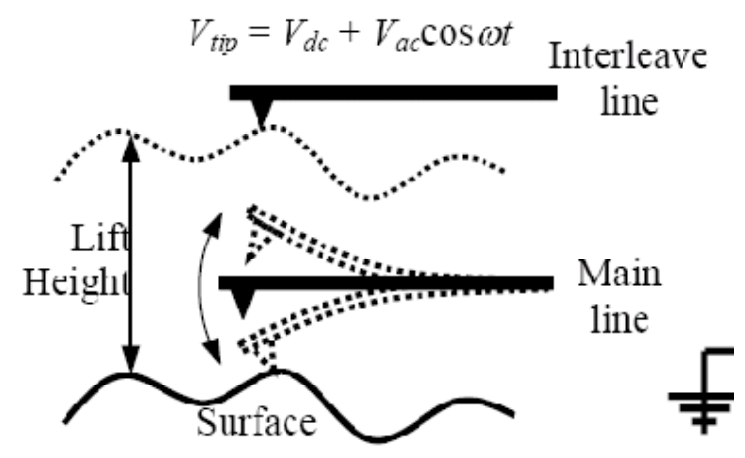

(a)

(b)

Figure 52: Schematic representation of SSPM operation [64].

$$
\begin{aligned}
& F_{d c}(z)=\frac{1}{2} \frac{\partial C(z)}{\partial z}\left[\left(V_{d c}-V_{\text {surf }}\right)^{2}+\frac{1}{2} V_{a c}^{2}\right], \\
& F_{1 \omega}(z)=\frac{\partial C(z)}{\partial z}\left(V_{d c}-V_{\text {saff }}\right) V_{a c}, \\
& F_{2 \omega}(z)=\frac{1}{4} \frac{\partial C(z)}{\partial z} \mathrm{~V}_{\mathrm{ac}}^{2} .
\end{aligned}
$$

Figure 53: The forces involved in SSPM [64]. 

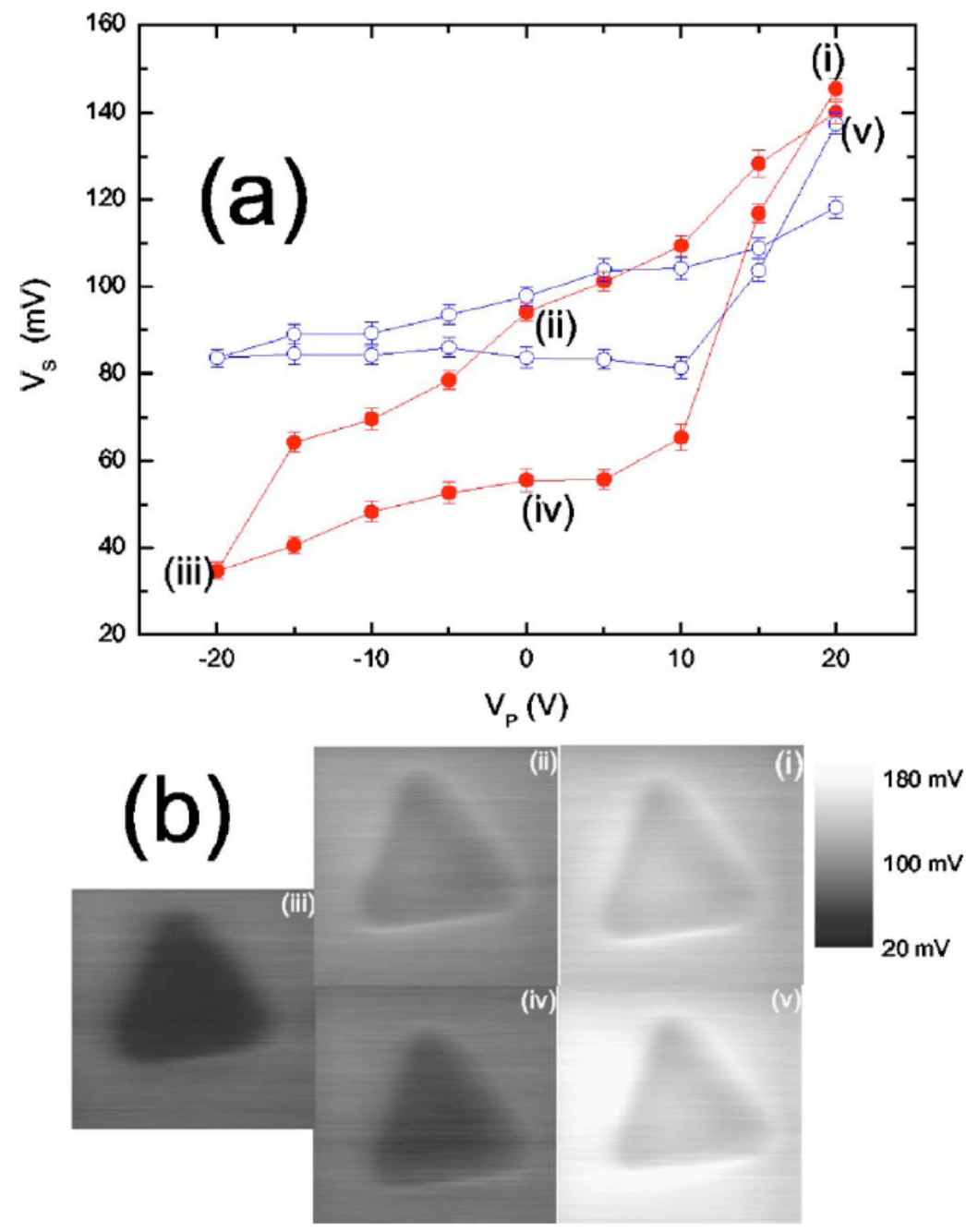

Figure 54: (a) Average surface potential $\left(V_{S}\right)$ as a function of poling voltage $\left(V_{P}\right)$ for a $44 \mathrm{~nm}$ tall, $500 \mathrm{~nm}$ sided prism (solid circle), and for the surrounding film area (hollow circle). (b) SSPM images for the data points labeled (i)-(v) in (a). The images are $1 \times 1 \mu \mathrm{m}^{2}$ and the surface potential scale is indicated in the legend. 


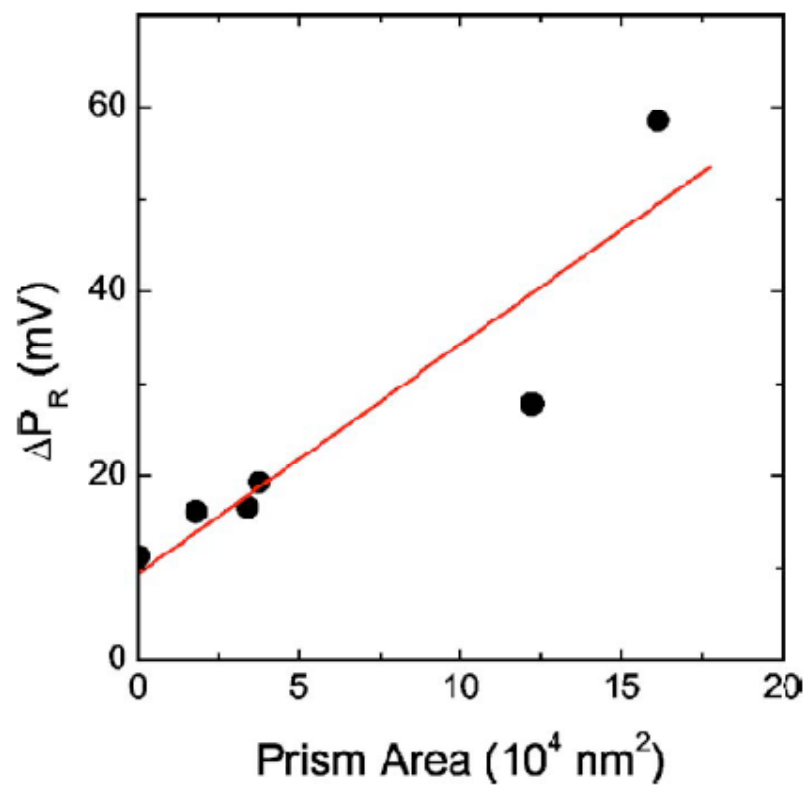

Figure 55: Remanent surface potential $V_{R}$ as a function of prism surface area. The red line is a fit of the data to a straight line with an intercept of $9.5 \mathrm{mV}$ and a slope of $2.5 \times 10^{-4} \mathrm{mV} / \mathrm{nm}^{2}$. 


\section{Prism \\ YMO \\ GaN}

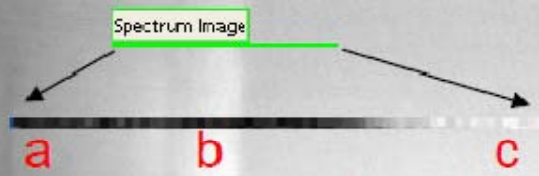

Spectrum imaging: you define a line or a 2D pixel grid and an EEL spectrum is acquired at every pixel. This way you scan/map the selected area.

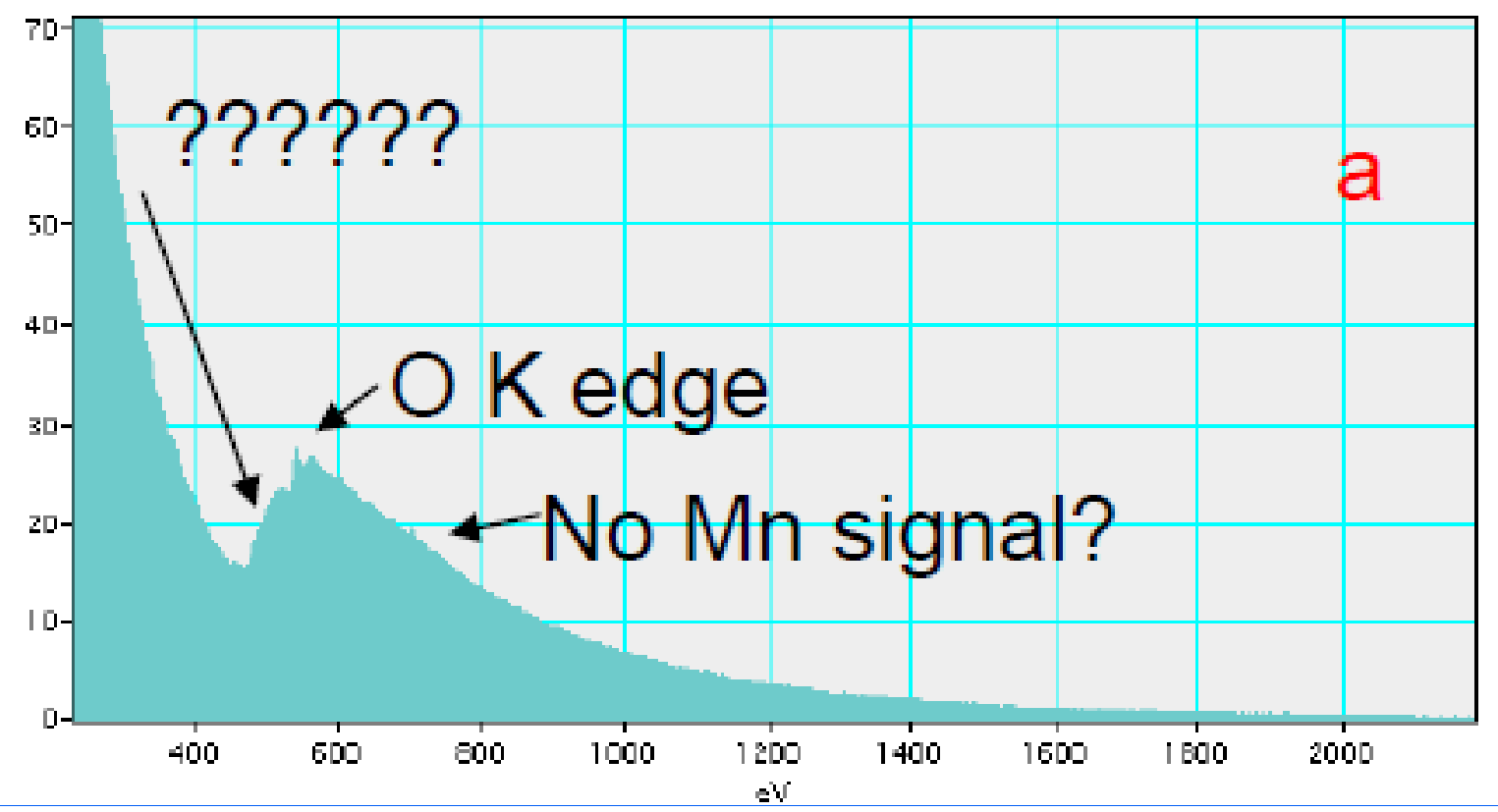

Figure 56: EELS scan position and spectrum for a location on one of the prism structures. The peak indicated by the "?" is located at the energy corresponding to In. 


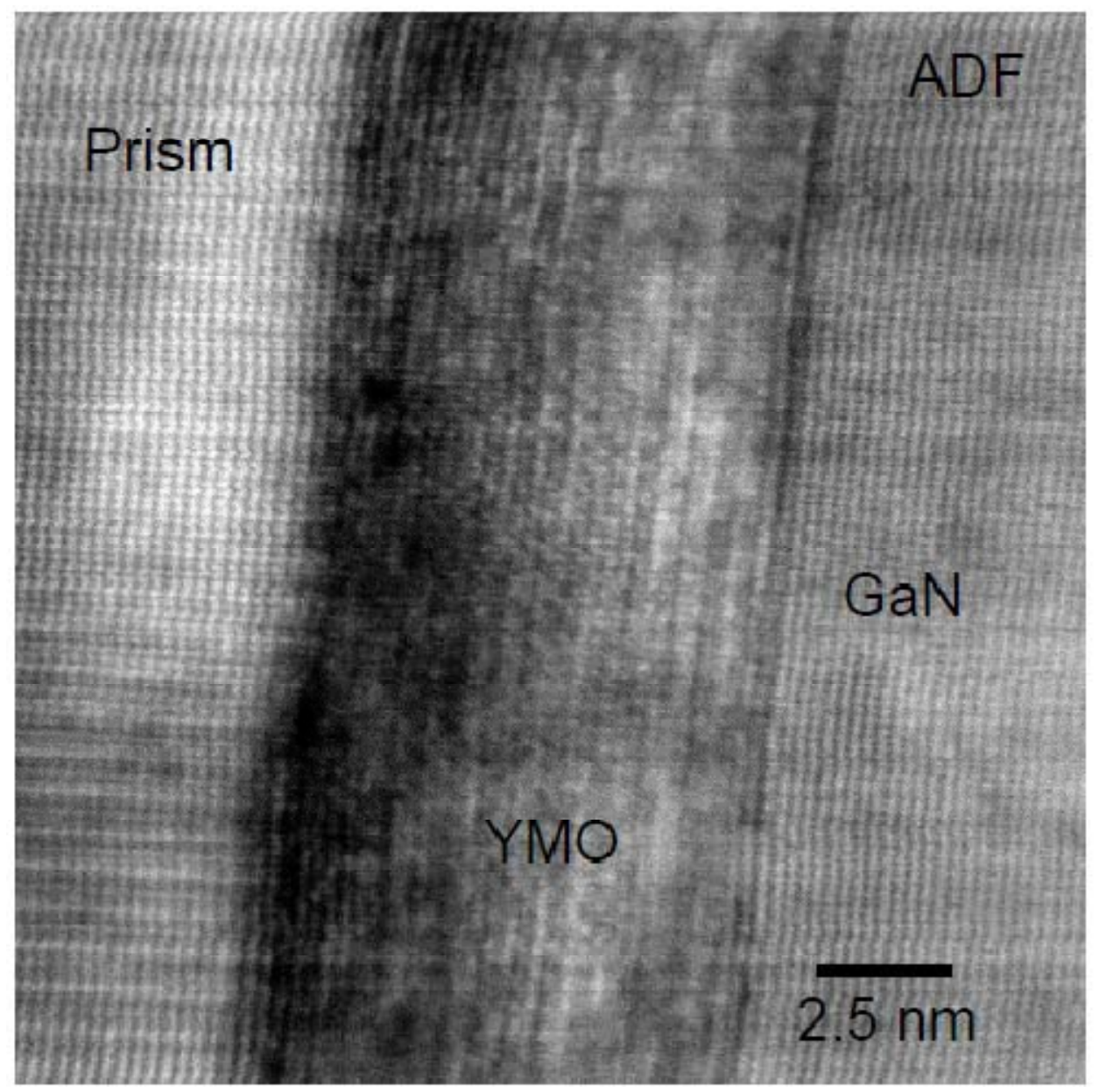

Figure 57: TEM image of the interface between the GaN, YMO, and a prism. The materials appear to be coherent. Note that rippling in the image is due to charging effects from the $\mathrm{Al}_{2} \mathrm{O}_{3}$. 


\section{Chapter 6: Summary}

Molecular beam epitaxy was used to grow $\mathrm{YMnO}_{3}$ on $\mathrm{GaN}$ with substrate deposition temperatures ranging from $750{ }^{\circ} \mathrm{C}$ to $900{ }^{\circ} \mathrm{C}$. Surface morphology, as determined by atomic force microscopy, indicated an increase in surface roughness from $3.4 \AA$ to $9 \AA$ as deposition temperature increased. X-ray reflectivity also indicated surface roughening with increasing temperature, but with a smaller increase, from $3.1 \AA$ to $4.9 \AA$. The lower bound placed via x-ray was from the 775 ${ }^{\circ} \mathrm{C}$ sample, as the $750{ }^{\circ} \mathrm{C}$ sample was polycrystalline and had high roughness.

Structural measurements using x-ray diffraction indicated that all samples grown above $750{ }^{\circ} \mathrm{C}$ were crystalline and displayed the epitaxial relations of YMO [001] $\| \mathrm{GaN}$ [001] and YMO [1-110] || GaN [110]. Lattice constants c and a were determined using the YMO (006) and YMO (106) or YMO (112) reflections. The c-axis lattice constants ranged from $11.39 \AA$ to $11.45 \AA$. The a-axis lattice constants ranged from $6.05 \AA$ to $6.25 \AA$. Rocking curve analysis of the YMO (002) reflections determined that the sample grown at $900{ }^{\circ} \mathrm{C}$ had the narrowest full width half maximum and therefore the highest crystallinity. X-ray reflectivity indicated that the samples included a layer of $\mathrm{GaO}$ between the $\mathrm{GaN}$ and YMO. As the temperature of deposition increased, the thickness of this layer increased from $5.6 \AA$ at $775^{\circ} \mathrm{C}$ to $104 \AA$ at $900{ }^{\circ} \mathrm{C}$.

Electrical measurements performed on metal ferroelectric semiconductor devices with Pt top contacts of area $400 \mu \mathrm{m}^{2}$ and thickness of $150.0 \mathrm{~nm}$. P-V measurements showed small ferroelectric response. $I-V$ measurements revealed 
that the samples had predominately three leakage mechanisms, ohmic conduction, space charge limited conduction, and Schottky emission. Space charge limited conduction was determined to have a exponential trap distribution. Estimates of the relative permittivity, while in the Schottky emission regime, ranged from 9 to 13, comparable to the value of 15 given by Huang et.al. Sample growth at $900{ }^{\circ} \mathrm{C}$ resulted in conduction due to ohmic and Fowler-Nordheim tunneling, indicating defects.

Unfortunately, even though the samples were epitaxial and crystalline, leakage effects dominated the electrical measurements. This essentially comprised the utility of the samples and made them unfit for memory retention. One way in which this may be circumvented is by the use of an insulating buffer layer, such as $\mathrm{Y}_{2} \mathrm{O}_{3}$, between the $\mathrm{GaN}$ and $\mathrm{YMO}$.

Future work will focus on the MBE growth of $\mathrm{YnO}_{3}$ on $\mathrm{GaN}$, as this material may be ferroelectric in thin films and can be grown in a perovskite or hexagonal structure, allowing for more flexibility in terms of crystal quality needed to maintain ferroelectricity. 


\section{Works Cited}

1 Scott, J. F. and Paz de Araujo, C. A. Science, 246 (1989), 1400.

2 Fujimura, N., Ishida, T., Yoshimura, T., and and Ito, T. Applied Physics Letters, 69 (1996), 1011.

3 Lines, M. E. and Glass, A. M. Principles and Applications of Ferroelectrics and Related Materials. Oxford University Press, Bristol, 1977.

4 Koster, G., Dimmock, J., Wheeler, R., and and Statz, H. Properties of the Thirty-Two Point Groups. M.I.T. Press, Cambridge, 1963.

5 R.Waser et al., eds. Polar Oxides. Wiley-VCH, 2005.

6 Kittel, Charles. Introduction to Solid State Physics. Wiley, Hoboken, 2005.

7 Blinc, R. and Zeks, B. Soft Modes in Ferroelectrics and Antiferroelectrics. American Elsevier, New York, 1974.

8 Cochran, W. Advan. Phys., 9 (1960), 387.

9 Cochran, W. Advan. Phys., 10 (1961), 401.

10 Anderson, P. W. Fizika dielektrikov (1959).

11 Thomas, R., Scott, J. F., Bose, D. N., and Katiyar, R. S. J. Phys.: Condens. Matter, 22 (2010), 423201 .

12 Hill, N. A. J. Phys. Chem. B, 104 (2000), 6694.

13 Imada, S., Shouriki, S., Tokumitsu, E., and Ishiwara, H. Jpn. J. Appl. Phys., 37 (1998), 64976501.

14 Ito, D., Fujimura, N., and Ito, T. Jpn. J. Appl. Phys., 39 (2000), 5525-5527.

15 Huang, Z. J., Cao, Y., Sun, Y. Y., Xue, Y. Y., and Chu, C. W. Phys. Rev. B, 56, 5 (1997), 2623-2626. 
16 Adem, U., Nugroho, A. A., Meetsma, A., and Palstra, T. T. M. Phys. Rev. B, 75 (2007), 014108.

17 Fennie, C. J. and Rabe, K. M. Phys. Rev. B, 72 (2005), 100103(R).

18 Lonkai, Th., Tomuta, D. G., Amann, U., Ihringer, J., Hendrikx, R. W. A., Tobbens, D. M., and Mydosh, J. A. Phys. Rev. B, 69 (2004), 134108.

19 Gibbs, A. S., S., Knight K., and Lightfoot, P. Phys. Rev. B, 83 (2011), 094111.

20 Fennie, C. J. and Rabe, K. M. Phys. Rev. B, 72, 100103(R) (2005).

21 van Aken, B. B., Palstra, T. T., Filipetti, M., and Spaldin, N. A. 2004. Nat. Mater., 3, 164.

22 Bertaut, E. F., Forrat, E. F., and Fang, P. H. C. R. Acad. Sci. Paris, 256 (1963), 1958-1961.

23 Isobe, M., Kimizuka, N., Nakamura, M., and Mohri, T. Acta Cryst., C47 (1991), 423-424.

24 Greedan, J. E., Bieringer, M., Britten, J. G., Giaquinta, D. M., and zur Loye, H.-C. J. Solid State Chem., 116 (1995), 118-130.

25 Pistorius, C. W. F. T. and Kruger, G. J. J. Inorg. Nucl. Chem., 38 (1976), 1471-1475.

26 Yakel, H. L., KOEHLER, W. C., Bertaut, E. F., and Forat, E. F. Acta Cryst., 16 (1963), 957.

27 Imada, S., Shouriki, S., Tokumitsu, E., and Ishiwara, H. Jpn. J. Appl. Phys., 37 (1998), 64976501.

28 Ito, D., Fujimura, N., and Ito, T. Jpn. J. Appl. Phys., 39 (2000).

29 Posadas, A., Yau, J.-B., Ahn, C. H. et al. Appl. Phys. Lett., 87 (2005).

30 Zywietz, T. K., Neugebauer, J., and Scheffler, M. Appl. Phys. Lett., 74, 12 (1999), 1695.

31 Chye, Y., Liu, T., Li, D., Lee, K., Lederman, D., and Myers, T. H. Appl. Phys. Lett., 88 (2006), 132903.

32 Wu, S. Y. IEEE Transactions on Electron Devices, ED-21, 8 (1974), 499.

$33 \mathrm{Kim}, \mathrm{K}$. H. IEEE ELECTRON DEVICE LETTERS, 19, 6 (1998).

34 Farrow, Robin F. C., ed. Molecular Beam Epitaxy. Noyes Publications, Park Ridge, 1995. 
35 Karsen, P.K. and Dobson, P. J., eds. Reflection High Energy Electron Diffraction and Reflection Electron Imaging of Surfaces. Plenum, NATO ASI Ser. B., 1988.

36 Svensson, S. P., Nilsson, P. O., and Andersson, T. B. Phys. Rev. B, 31 (1985), 5272.

37 Rzhanov, A. V. and Iljina, L. A., eds. Ellipsometry: Theory, Methods, Applications. Nauka, 1987.

38 Sitter, H., Glanner, G. J., and Herman, M. A. Vacuum, 46 (1995), 69.

39 Romano, L. T., Northrup, J. E., Ptak, A. J., and Myers, T. H. Appl. Phys. Lett., 77, 16 (2000), 2479-2482.

40 Trampert, A. and Ploog, K. H. Cryst. Res. Technol., 35, 6-7 (2000), 793-806.

41 Nakamura, S. and Mukai, T. Jpn. J. Appl. Phys., 31 (1992), L1457-L1459.

42 Heffernan, J., Kauer, M., Johnson, K., Zellweger, C., Hooper, S. E., and Bousquet, V. Phys. Stat. Sol. (a), 202 (2005), 868-874.

43 Herman, M. A. and Sitter, H. Molecular Beam Epitaxy. Springer, Heidelberg, 1996.

44 Schlom, D. Molecular Beam Epitaxial Growth of Cuprate Superconductors and Related Phases. 1990.

45 Singh, Jasprit. Electronic and Optoelectronic Properties of Semiconductor Structures. Cambrige University Press, New York, 2003.

46 Dobson, P. J., Joyce, B. A., Neave, J. H., and Zhang, J. J. Cryst. Growth, 81 (1987), 1.

47 Warren, B. E. X-Ray Diffraction. Dover Publications, Mineola, 1990.

48 Myers, H. P. Introductory Solid State Physics. Taylor-Francis, Philadelphia, 1997.

49 Giacovazzo, C., ed. Fundamentals of Crystallography. Oxford Science Publications, New York, 2002.

50 Fullerton, E., Schuller, I. K., Vanderstraetan, H., and Bruynseraede, Y. Phys. Rev. B, 45, 16 (1992), 9292-9310.

51 Gibaud, A. and Hazra, S. Current Science, 78, 12 (2000), 1467-1477.

52 Parratt, L. G. Phys. Rev., 95 (1954), 359. 
53 Névot, L. and Croce, P. Rev. Phys. Appl., 15 (1980), 761.

54 Thornton, J. Scanning Probe Microscopy Training Notebook. Veeco, 2000.

55 Sawyer, C. B. and Tower, C. H. Physical Review, 35 (1930), 269.

56 RADIANT TECHNOLOGIES. Ferroelectric Test System Manual. 2009.

57 Brooks, C. M., Fitting Kourkouti, L., Heeg, T., Schubert, J., Muller, D. A., and Schlom, D. G. Appl. Phys. Lett., 94 (2009), 162905.

58 Björk, M. and Andersson, G. Journal of Applied Crystallography, 40 (2007), 1174.

59 Sze, S. M. and Ng, K. K. Physics of Semiconductor Devices. Wiley India, New Delhi, 2007.

60 Kim, Y., Ohmi, S., Tsusui, K., and Iwai, H. Jpn. J. Appl. Phys., 44, 6A (2005), 4032.

61 Huang, Z. J., Cao, Y., Sun, Y. Y., Xue, Y. Y., and Chu, C. W. Phys. Rev. B, 56 (1997), 2623.

62 L., Wu. C., Shen, C. H., and Gwo, S. Appl. Phys. Lett., 88 (2006), 032105.

63 Green, J. C. Ann. Rev. Phys. Chem., 28 (1977), 161-83.

64 Kalinin, S. V. and Bonnell, D. A. In Alexe, M. and Gruverman, A, eds., Nanoscale Characterization of Ferroelectric Materials. Springer, 2004.

65 Keenan, C, Chandril, S., Myers, T. H., Moore, E. R., Cabrera, A. L., and Lederman, D. Appl. Phys. Lett., 92 (2008), 013119.

66 Abrahams, S. C. Acta Cryst., B57 (2001), 485-490.

67 Aken, B. B. van, Meetsma, A., and Palstra, T. T. M. Acta Cryst., C57 (2001), 230-232.

68 Zhong, C., Jiang, X., Yu, H., Jiang, Q., Fang, J., and Li, Z. Journal of Magnetism and Magnetic Materials, 321 (2009), 1260-1265.

69 Honig, R. E. and Kramer, D. A. RCA Review, 30 (1969), 285.

70 Yoshimura, T., Fujimura, N., Aoki, N., Hokayama, K., Tsuki, S., Kawabata, K., and Ito, T. Jpn. J. Appl. Phys., 36 (1997), 5921-5924.

71 Cho, A. Y. Thin Solid Films, 100 (1983), 291. 
72 Hong, S., ed. Nanoscale Phenomena in Ferroelectric Thin Films. Luwer Academic Publishers, Norwell, 2004.

73 Dvorak, J. and Petzelt, J. Phys. Lett., 35A (1971), 209.

74 Dawber, M., Rabe, K. M., and Scott, J. F. Rev. Mod. Phys., 77, 4 (2005), 1083. 


\section{Appendix}

\section{A.1 RHEED Results}

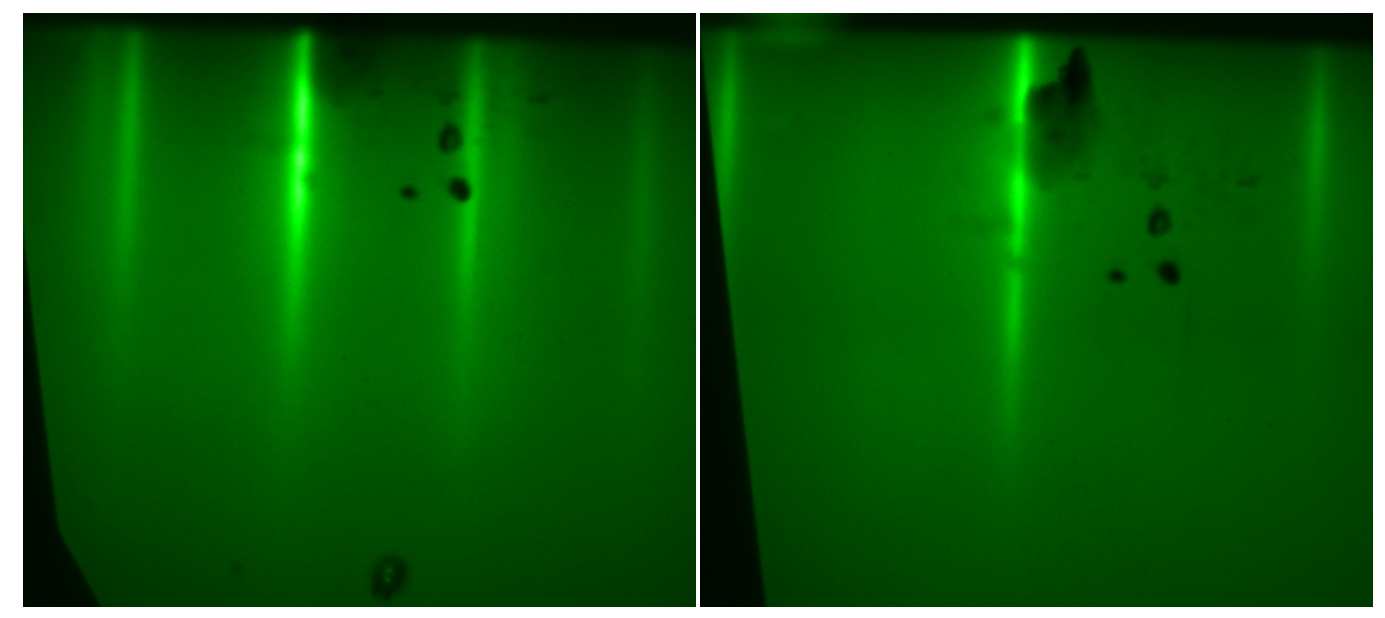

Figure 58: RHEED for sample grown at $775^{\circ} \mathrm{C}$. (Left) RHEED [110] lattice reflection. (Right) RHEED [100] lattice reflection.

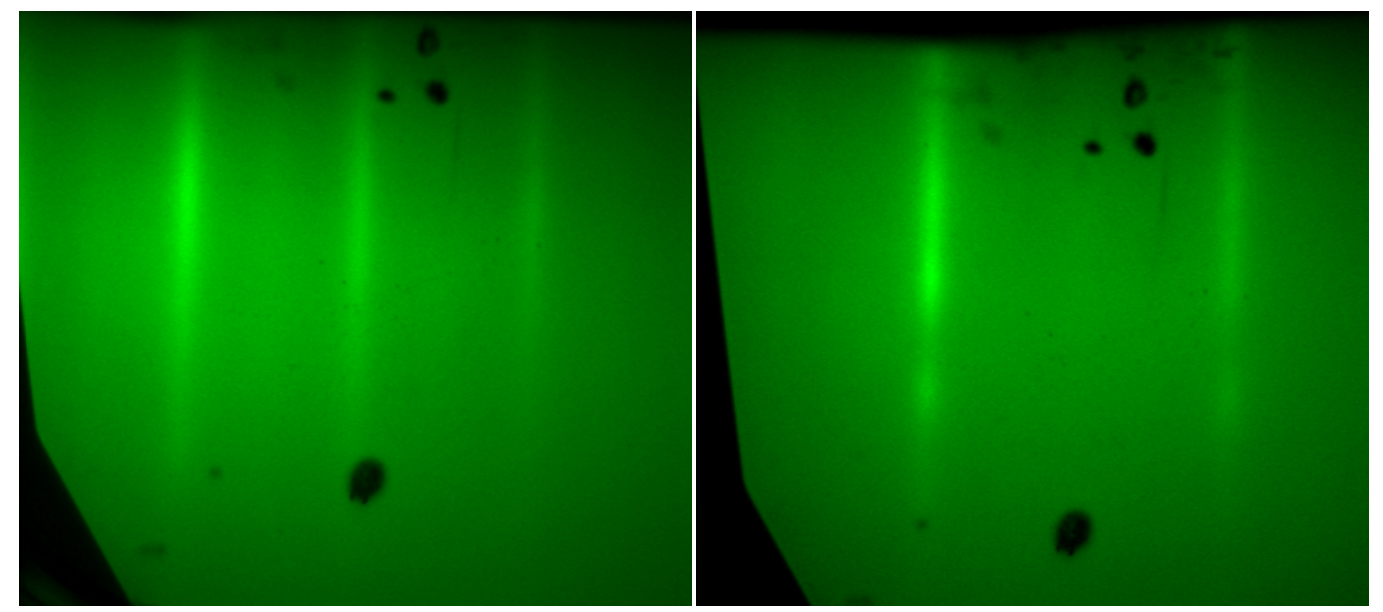

Figure 59: RHEED for sample grown at $800{ }^{\circ} \mathrm{C}$. (Left) RHEED [110] lattice reflection. (Right) RHEED [1-10] lattice reflection. 


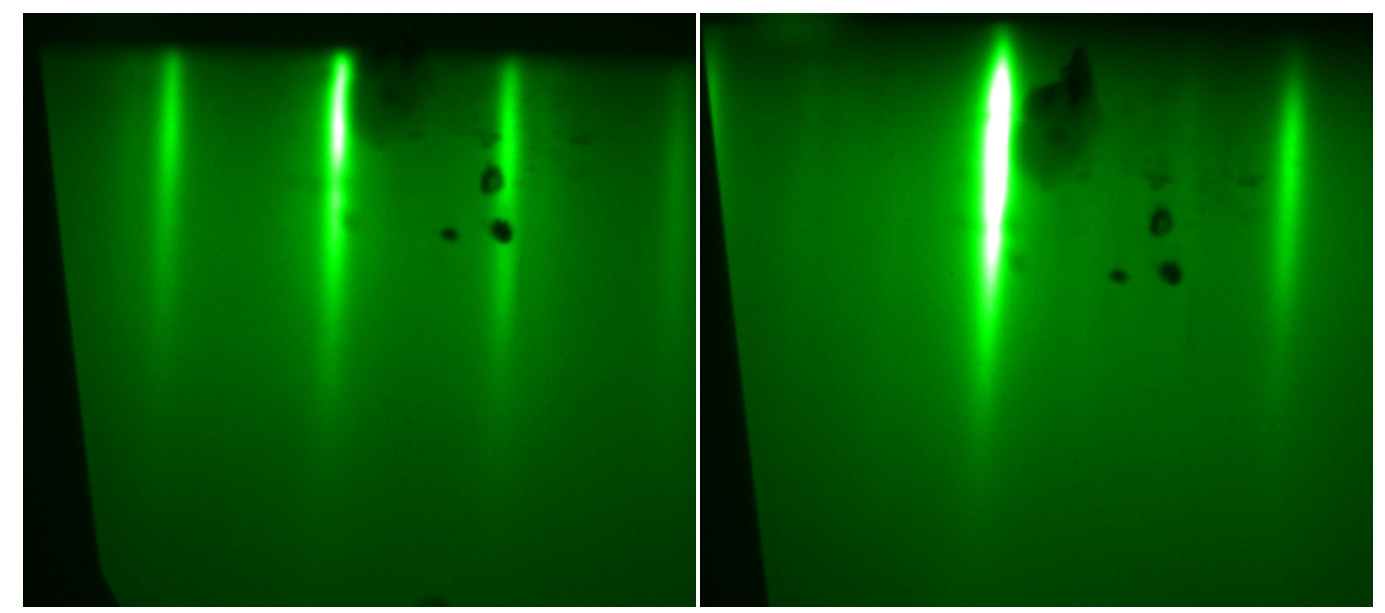

Figure 60: RHEED for sample grown at $825^{\circ} \mathrm{C}$. (Left) RHEED [110] lattice reflection. (Right) RHEED [1-10] lattice reflection.

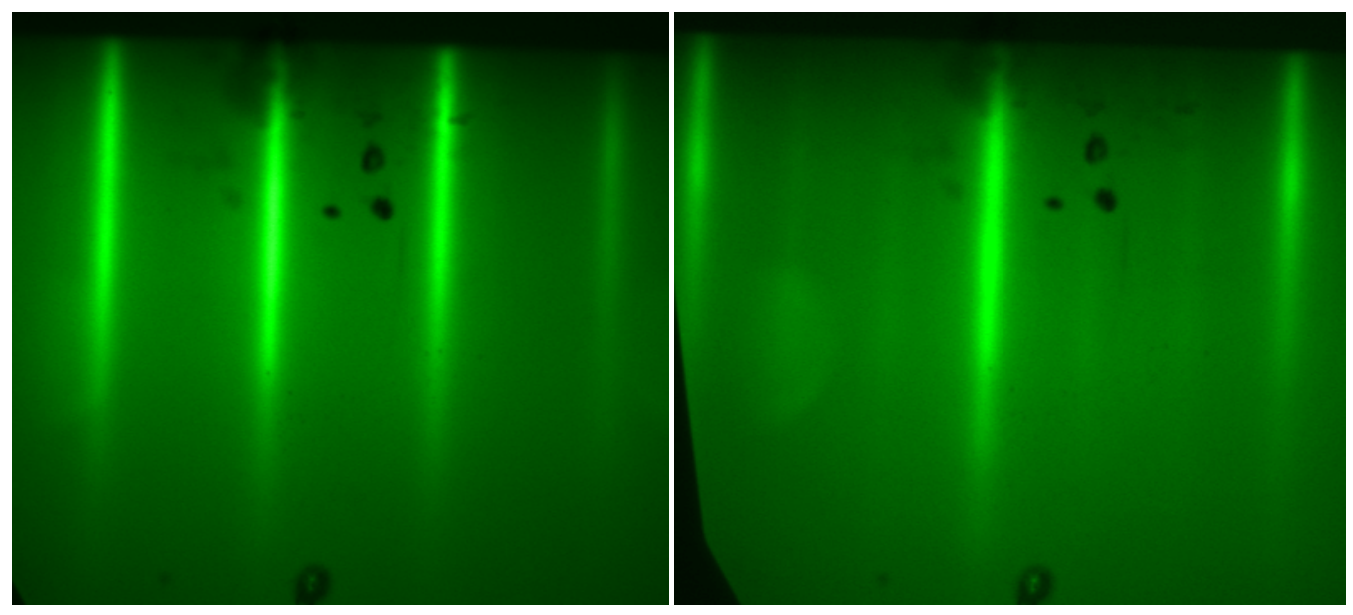

Figure 61: RHEED for sample grown at $850{ }^{\circ} \mathrm{C}$. (Left) RHEED [110] lattice reflection. (Right) RHEED [1-10] lattice reflection. 


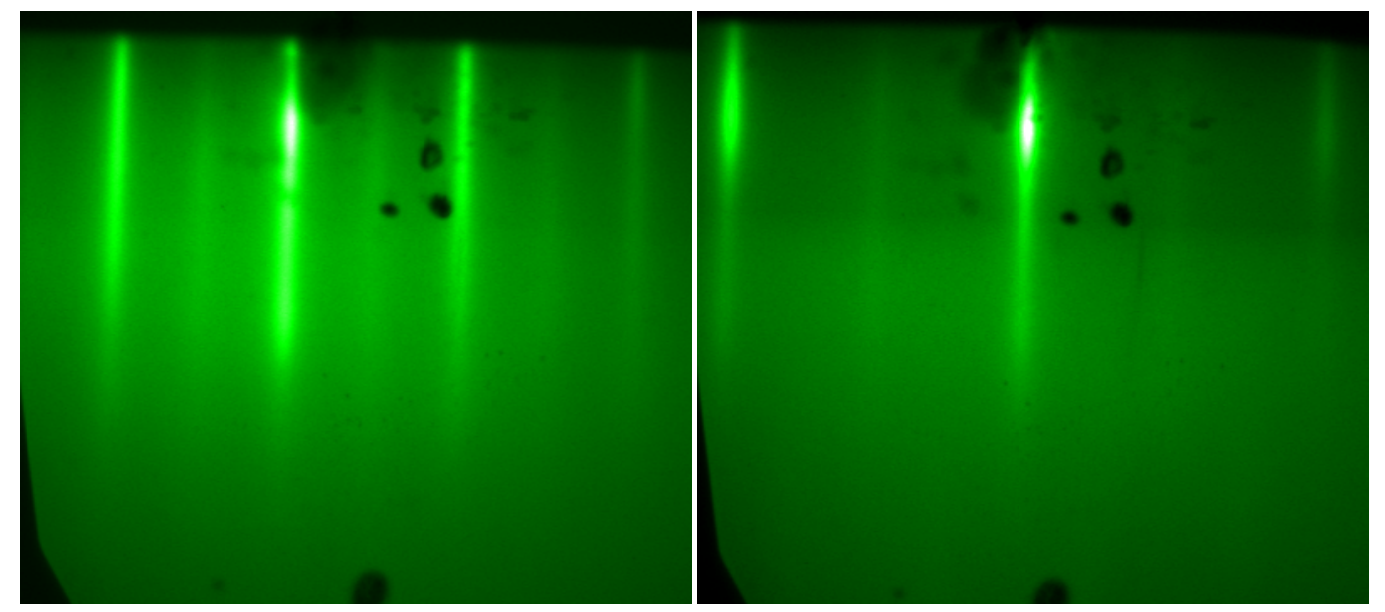

Figure 62: RHEED for sample grown at $875^{\circ} \mathrm{C}$. (Left) RHEED [110] lattice reflection. (Right) RHEED [1-10] lattice reflection.

\section{A.2 AFM Results}

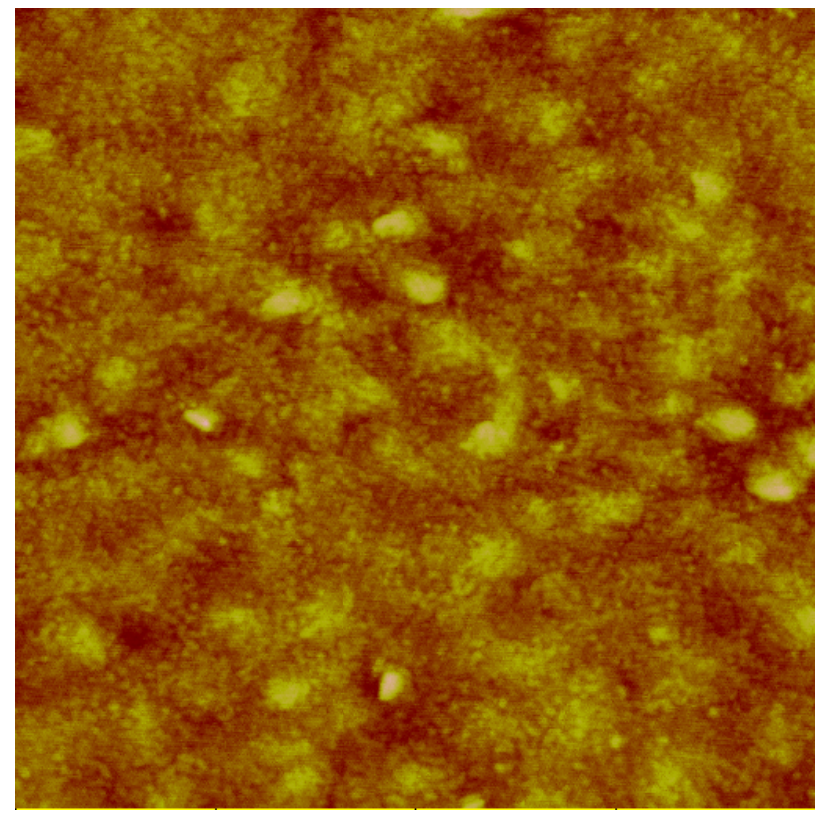

Figure 63: $1 \mu \mathrm{m}^{2}$ AFM image of sample grown at $775^{\circ} \mathrm{C}$. RMS roughness was estimated to be $3.5 \AA$. 


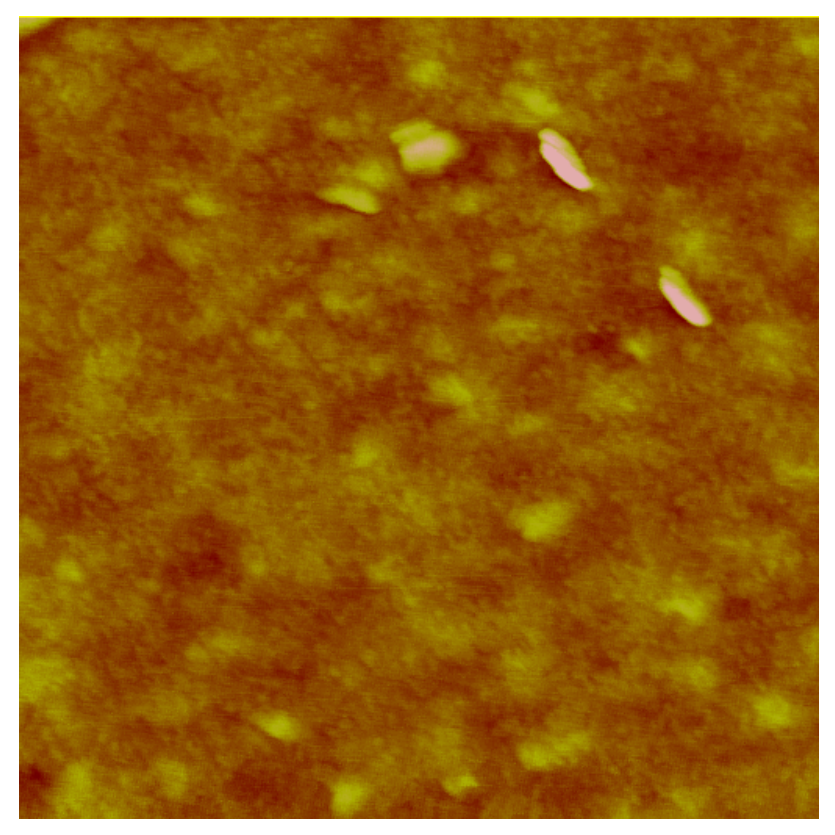

Figure 64: $1 \mu \mathrm{m}^{2}$ AFM image of sample grown at $800{ }^{\circ} \mathrm{C}$. RMS roughness was estimated to be $4.1 \AA$.

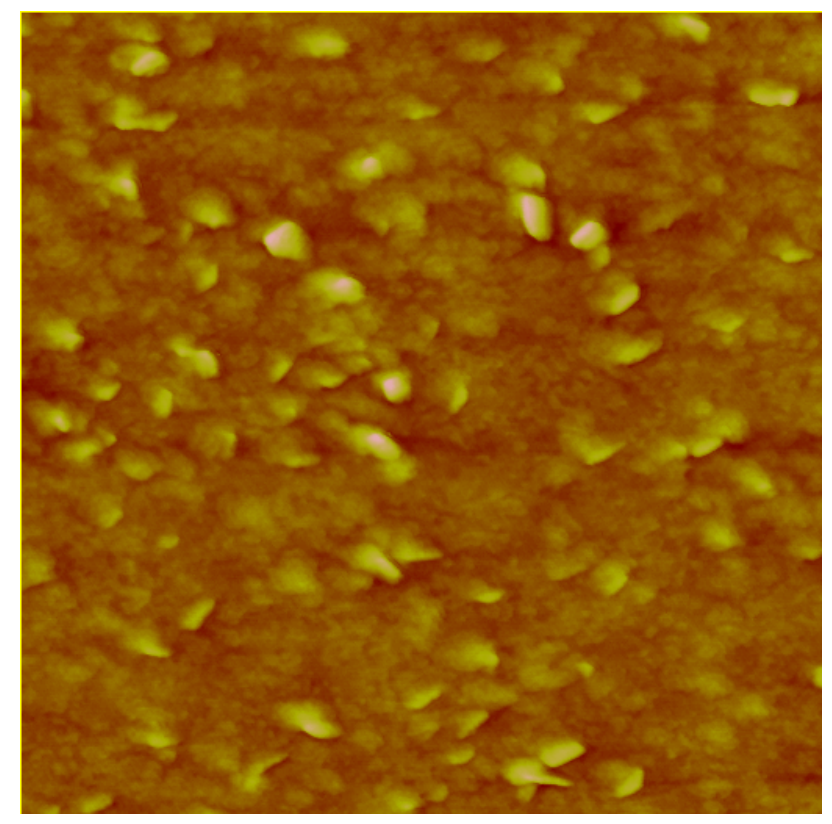

Figure 65: $1 \mu \mathrm{m}^{2}$ AFM image of sample grown at $825{ }^{\circ} \mathrm{C}$. RMS roughness was estimated to be $5.8 \AA$. 


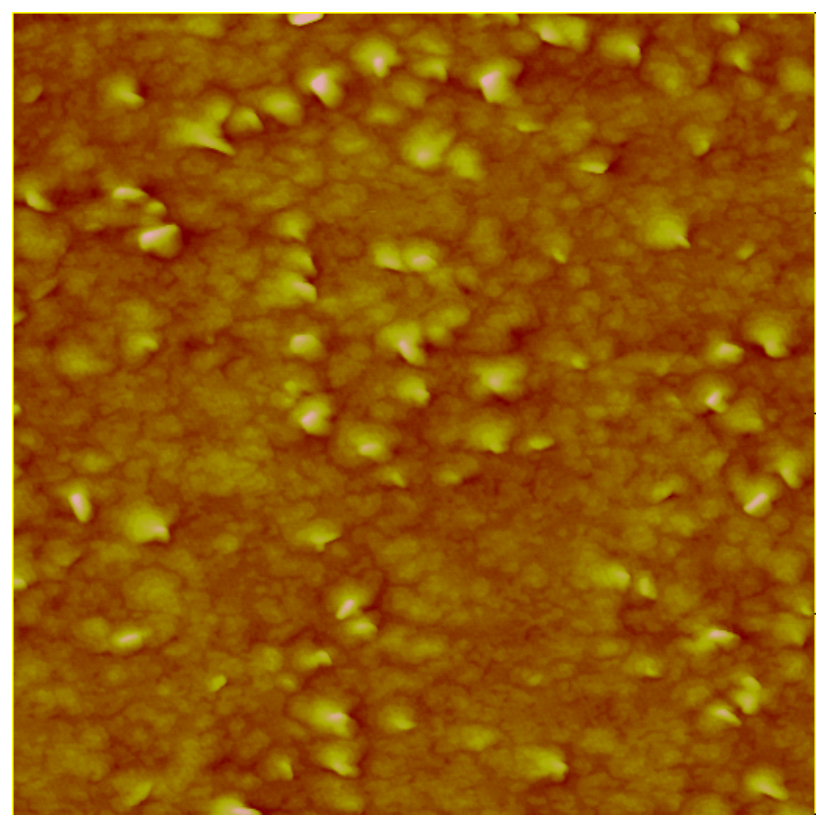

Figure 66: $1 \mu \mathrm{m}^{2}$ AFM image of sample grown at $850{ }^{\circ} \mathrm{C}$. RMS roughness was estimated to be $6.4 \AA$.

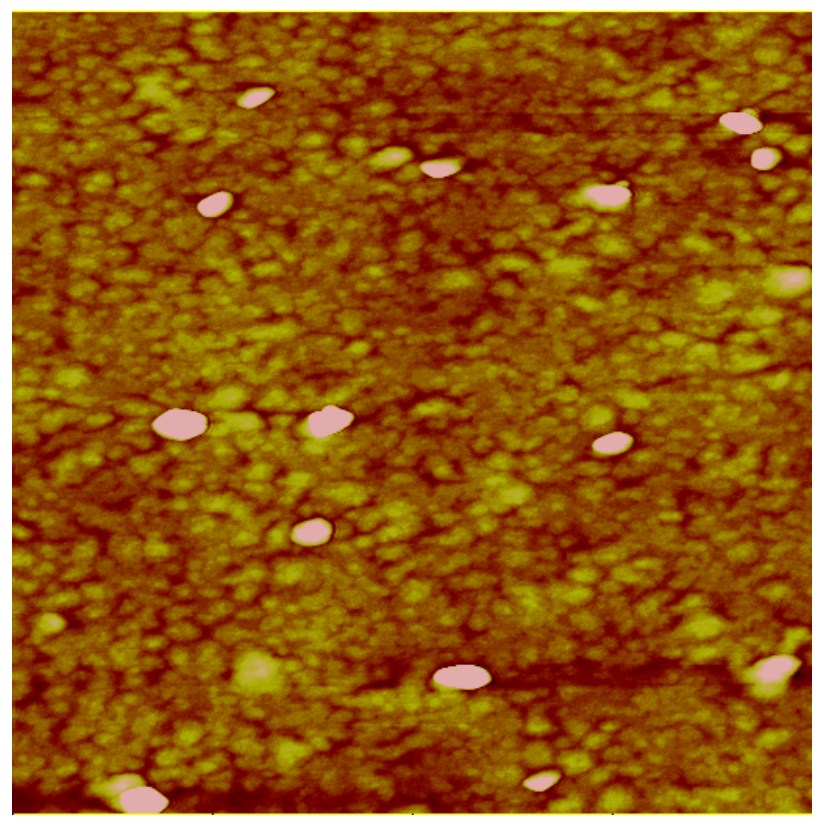

Figure 67: $1 \mu \mathrm{m}^{2}$ AFM image of sample grown at $875^{\circ} \mathrm{C}$. RMS roughness was estimated to be $9.4 \AA$. 


\section{A.3 X-Ray Diffraction Results}

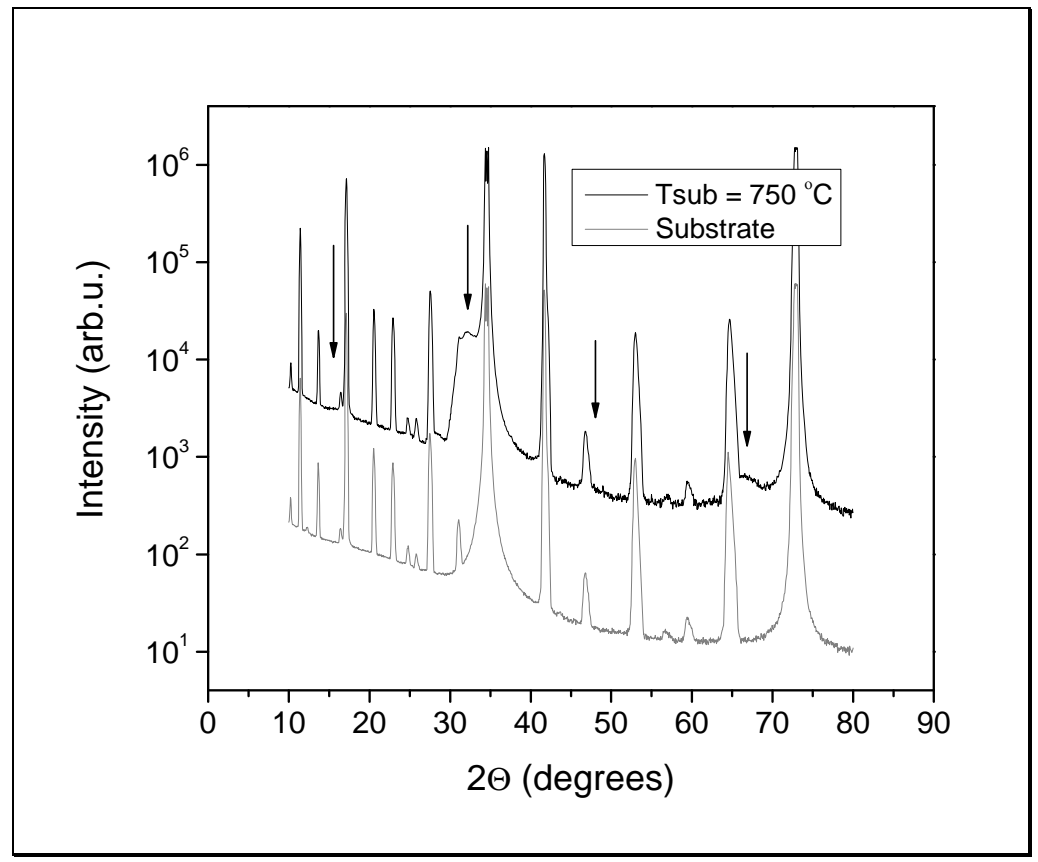

Figure 68: X-ray diffraction of $\mathrm{YMO}$ grown at $750{ }^{\circ} \mathrm{C}$. Arrows indicate the expected positions of the YMO $(00 l)$ reflections.

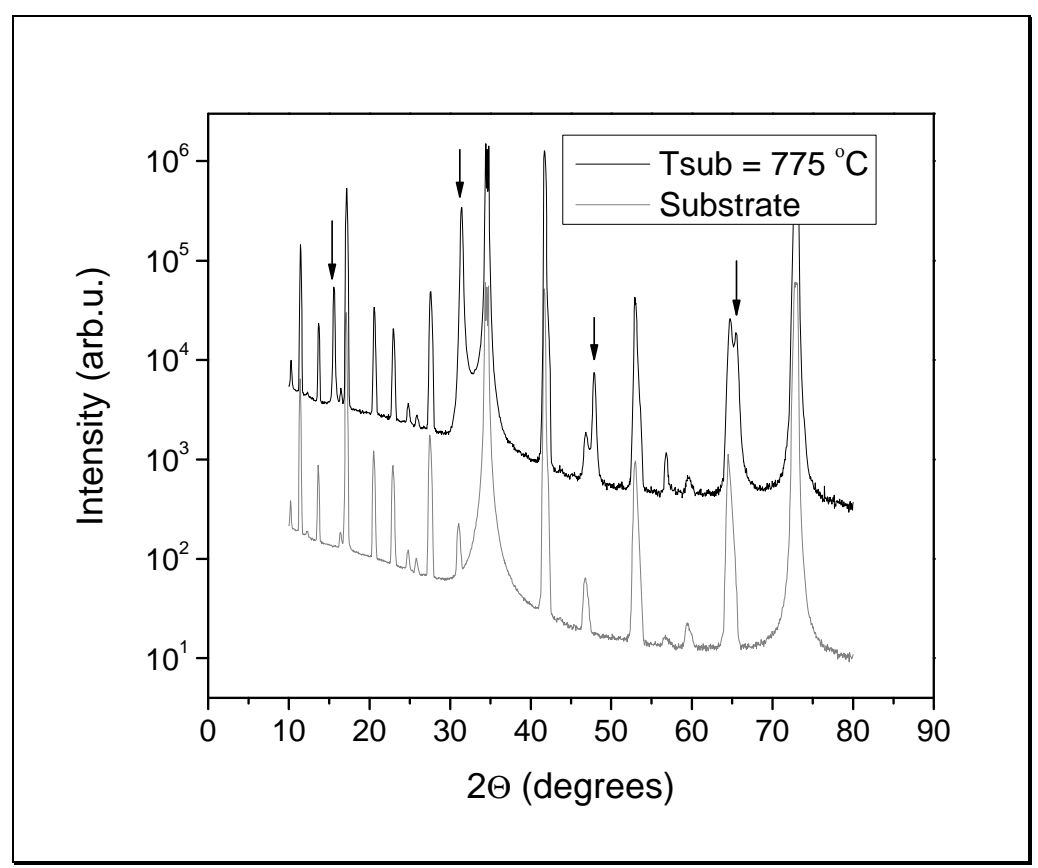

Figure 69: $\mathrm{X}$-ray diffraction of sample grown at $775{ }^{\circ} \mathrm{C}$. Arrows indicate the YMO $(00 l)$ reflections. All YMO $(00 l)$ reflections are strong and no other phases are observed. 


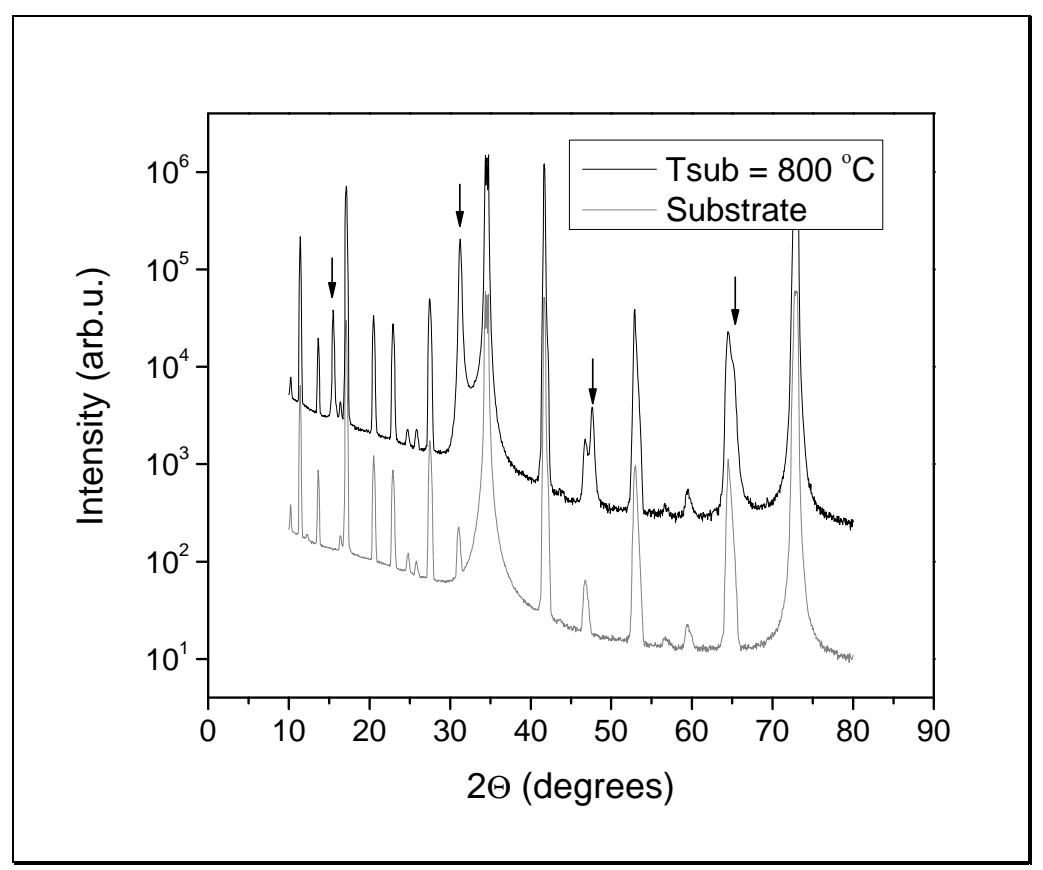

Figure 70: $\mathrm{X}$-ray diffraction of sample grown at $800{ }^{\circ} \mathrm{C}$. Arrows indicate the YMO $(00 l)$ reflections. All YMO $(00 l)$ reflections are strong and no other phases are observed.

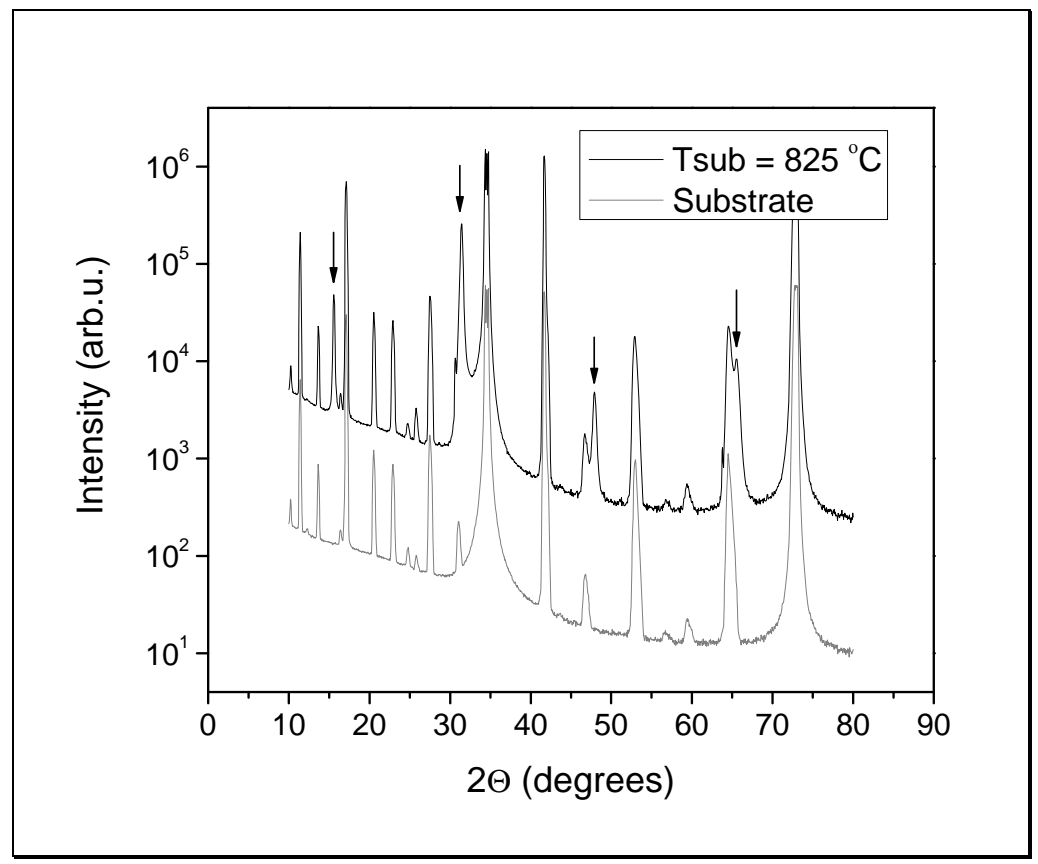

Figure 71: X-ray diffraction of sample grown at $825^{\circ} \mathrm{C}$. Arrows indicate YMO $(00 l)$ reflections. All YMO $(00 l)$ reflections are strong. Unidentifiable peaks are located to low angle side of both the (004) and (008) reflections. 


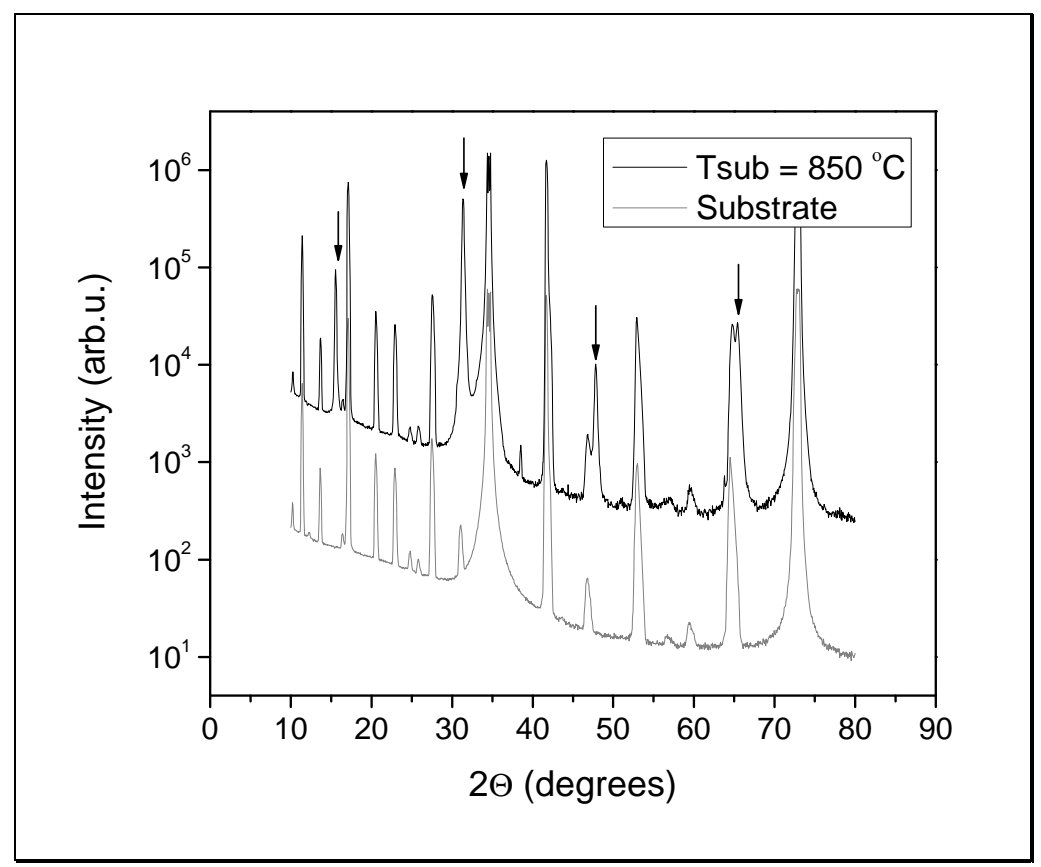

Figure 72: $\mathrm{X}$-ray diffraction of sample grown at $850{ }^{\circ} \mathrm{C}$. Arrows indicate $\mathrm{YMO}$ $(00 l)$ reflections. All YMO $(00 l)$ reflections are strong. Unidentifiable peaks are located to low angle side of both the (004) and (008) reflections.

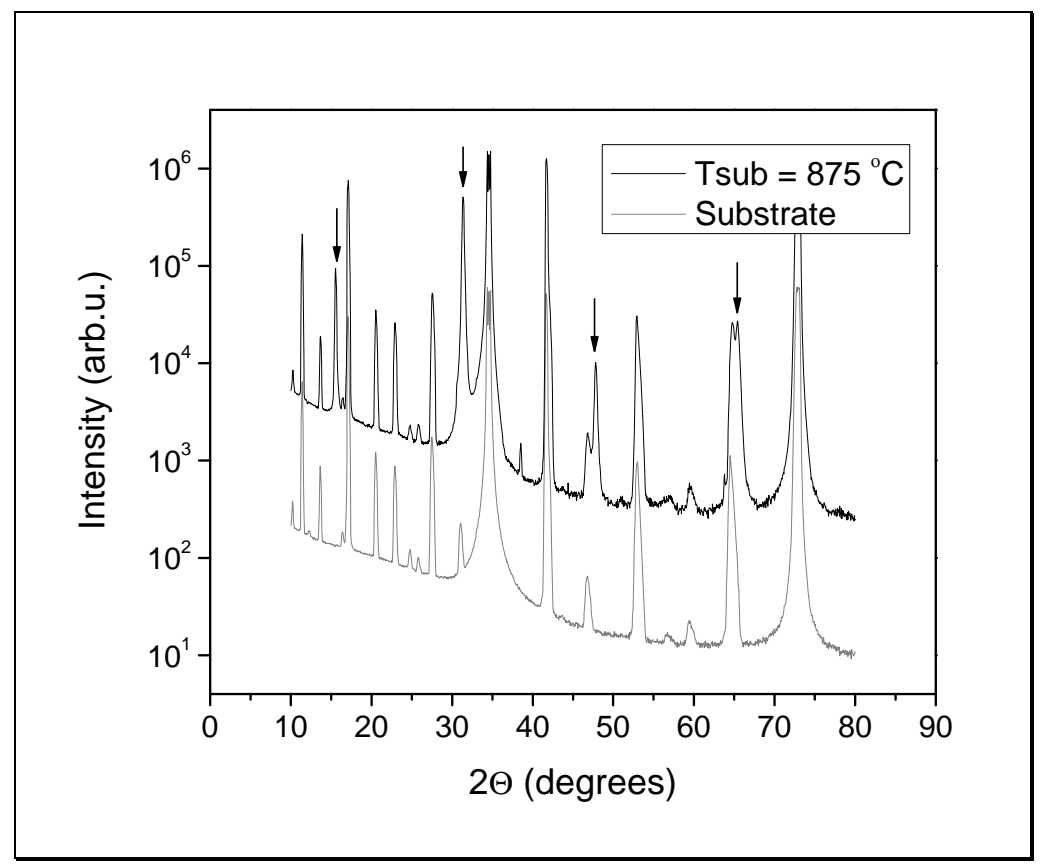

Figure 73: X-ray diffraction of sample grown at $875^{\circ} \mathrm{C}$. Arrows indicate $\mathrm{YMO}$ $(00 l)$ reflections. All YMO $(00 l)$ reflections are strong. Unidentifiable peaks are located to low angle side of both the (004) and (008) reflections. 


\section{A.4 Fits of the YMO(006) reflection}

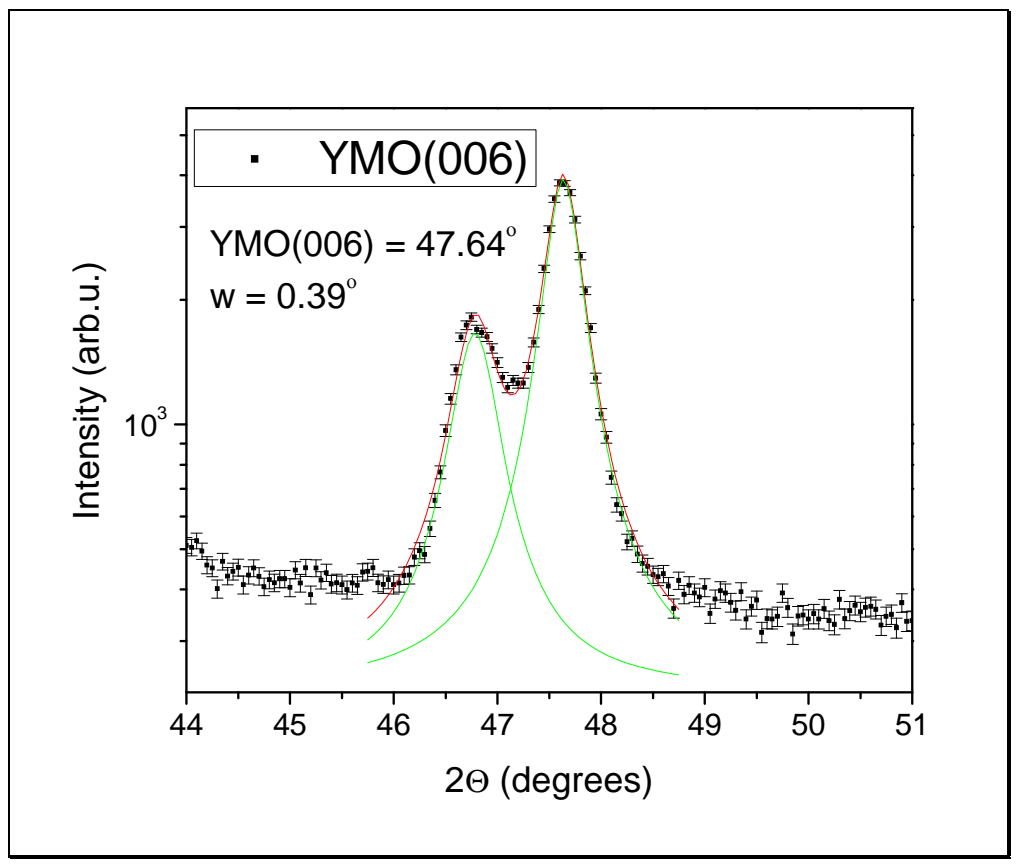

Figure 74: Lorentzian fit of the YMO (006) peak and nearby substrate peak. Scatter markers denote the measured data along with the associated error. Solid lines indicate the fit to each peak and the combined fit $\left(800^{\circ} \mathrm{C}\right)$.

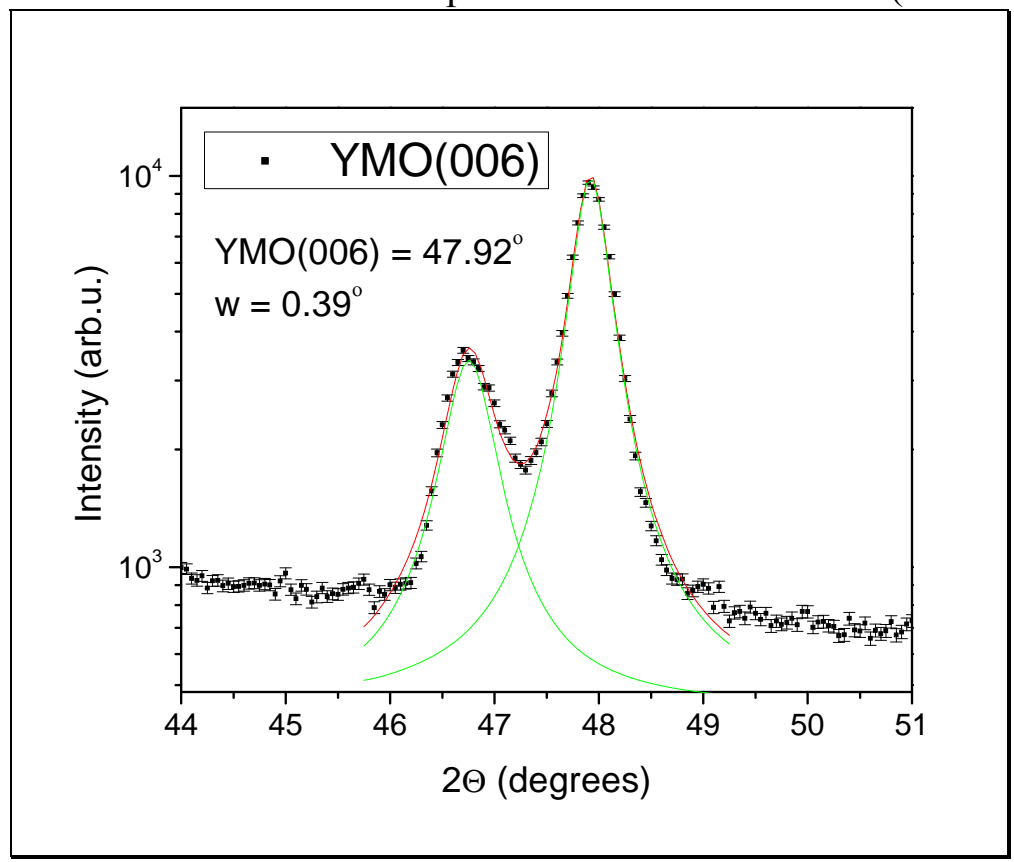

Figure 75: Lorentzian fit of the YMO (006) peak and nearby substrate peak. Scatter markers denote the measured data along with the associated error. Solid lines indicate the fit to each peak and the combined fit $\left(825^{\circ} \mathrm{C}\right)$. 


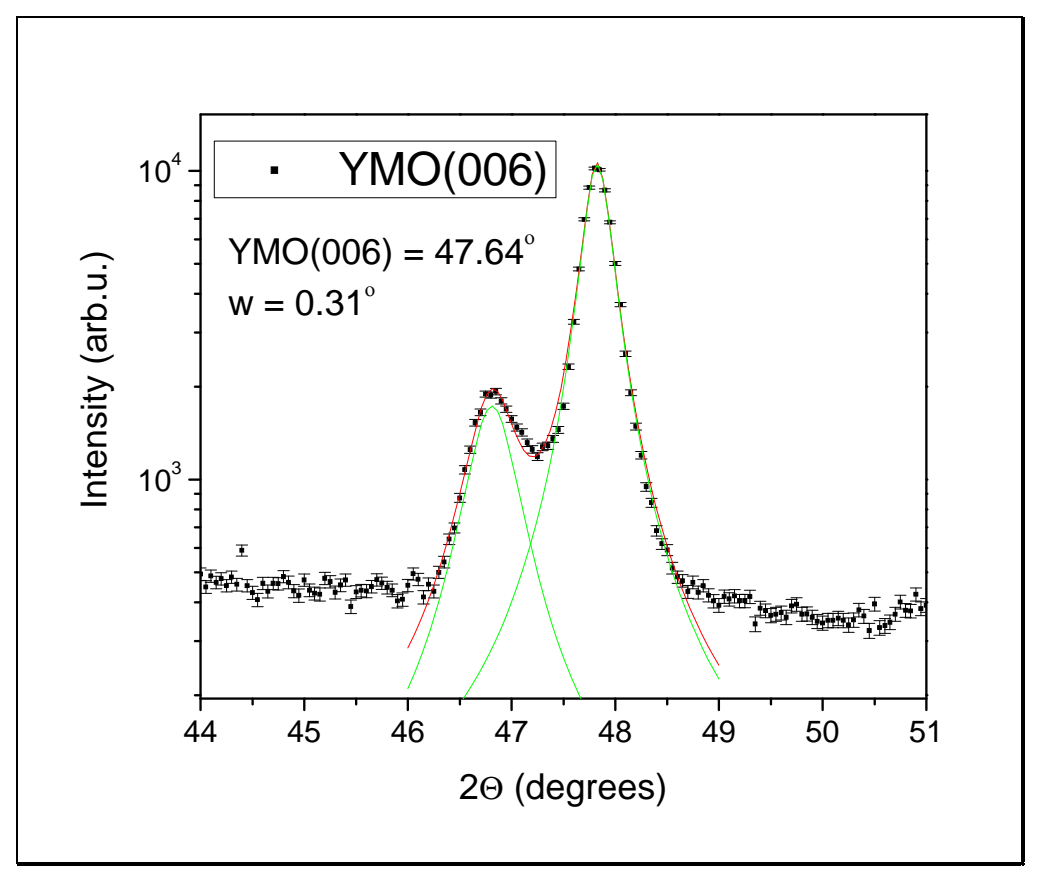

Figure 76: Lorentzian fit of the YMO (006) peak and nearby substrate peak. Scatter markers denote the measured data along with the associated error. Solid lines indicate the fit to each peak and the combined fit $\left(850^{\circ} \mathrm{C}\right)$.

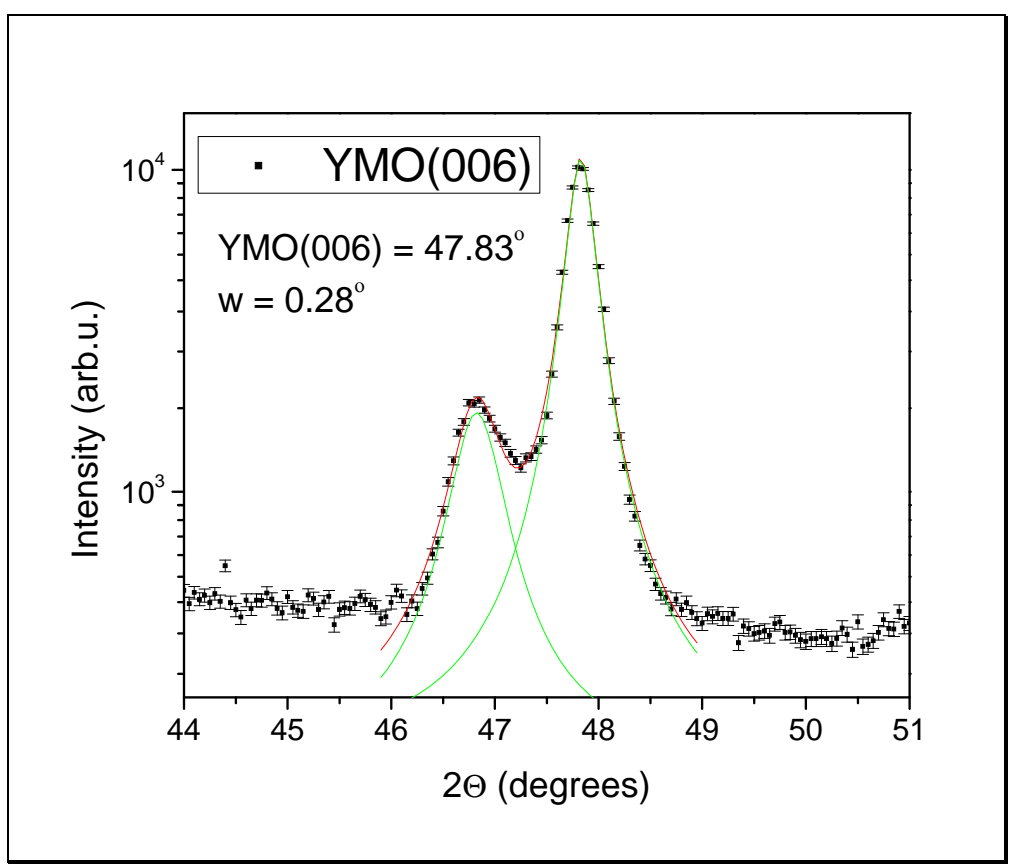

Figure 77: Lorentzian fit of the YMO (006) peak and nearby substrate peak. Scatter markers denote the measured data along with the associated error. Solid lines indicate the fit to each peak and the combined fit $\left(875^{\circ} \mathrm{C}\right)$. 


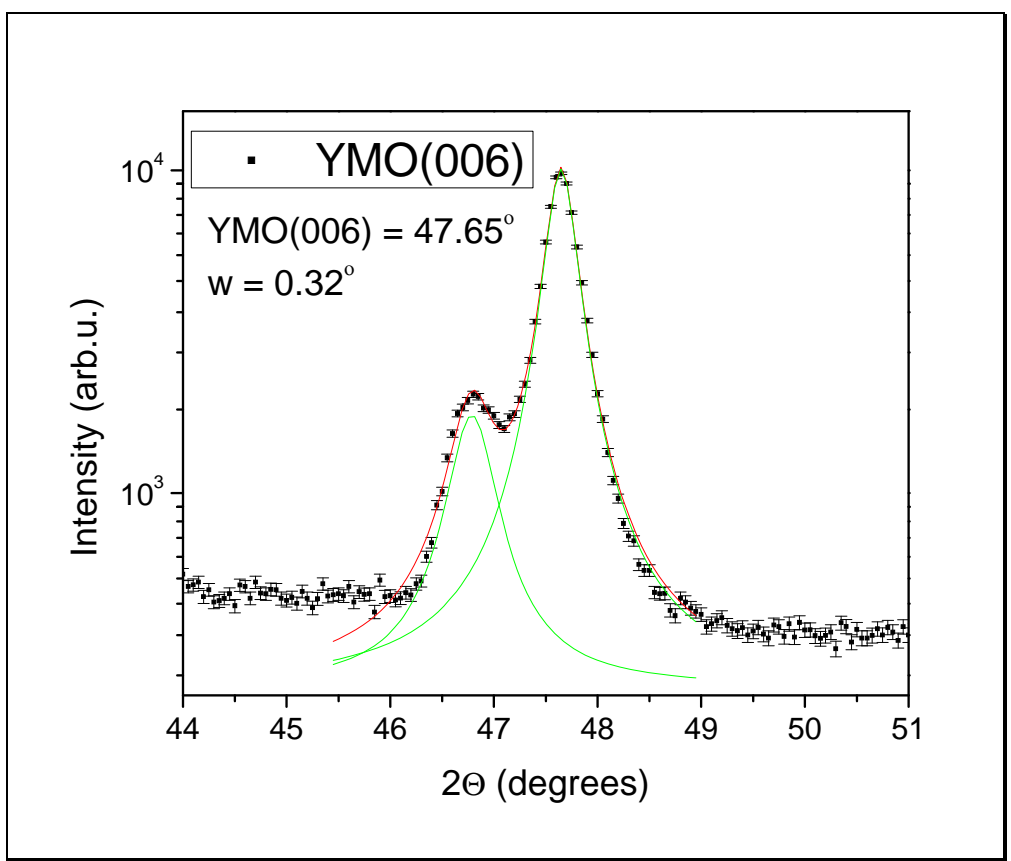

Figure 78: Lorentzian fit of the YMO (006) peak and nearby substrate peak. Scatter markers denote the measured data along with the associated error. Solid lines indicate the fit to each peak and the combined fit $\left(900{ }^{\circ} \mathrm{C}\right)$. 


\section{A.5 Rocking Curves of YMO (002)}

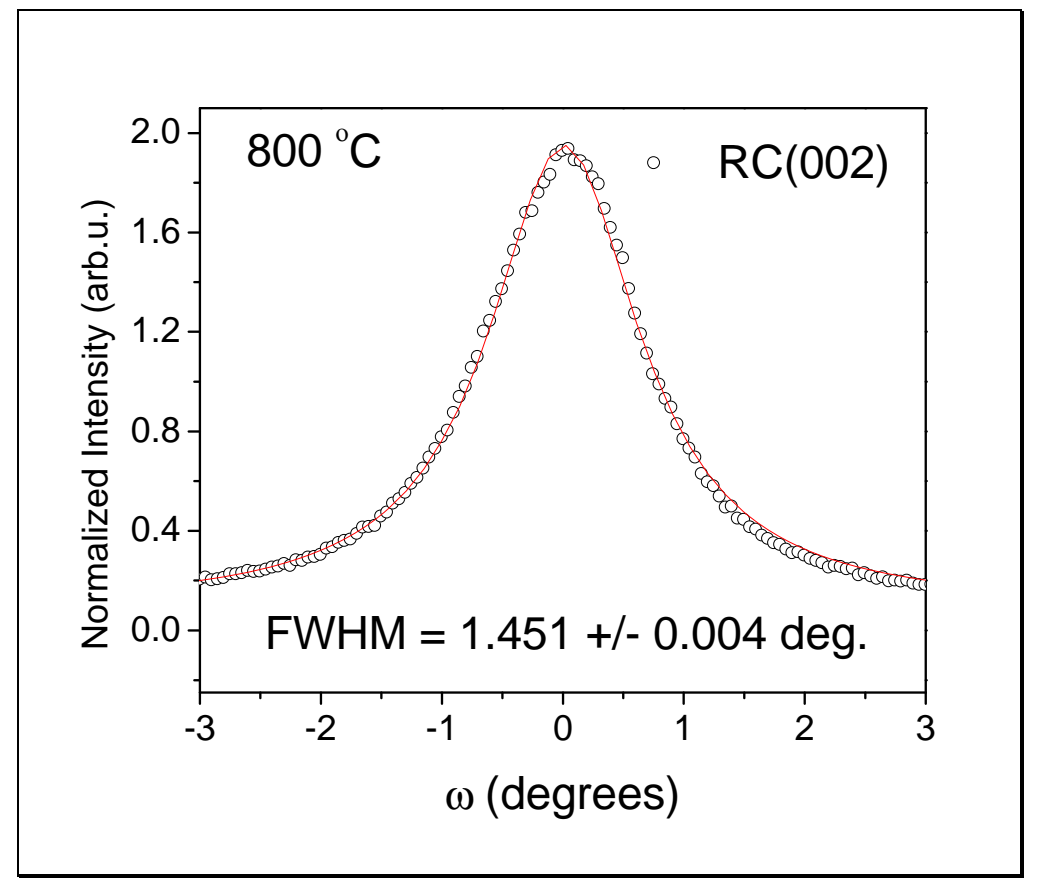

Figure 79: Rocking curve of YMO (002) reflection. Circles indicate measured data and the solid line a lorentzian fit $\left(800 \mathrm{C}^{\circ}\right)$.

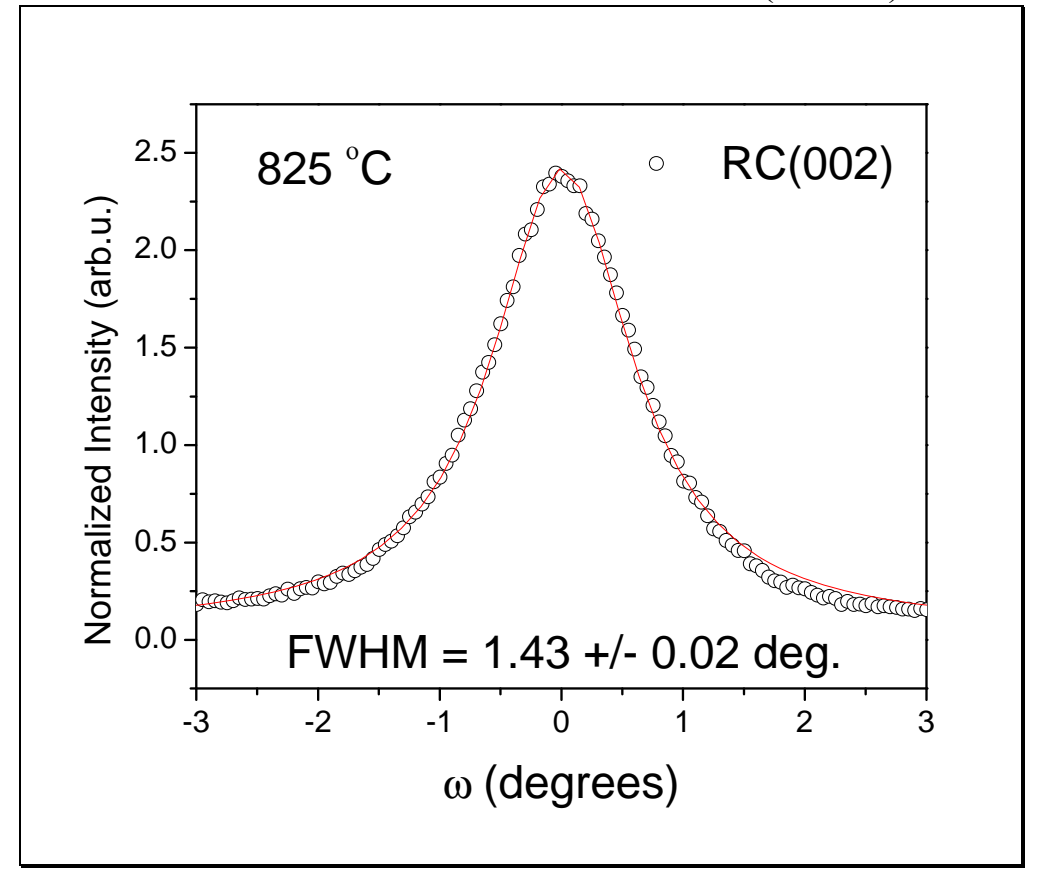

Figure 80: Rocking curve of YMO (002) reflection. Circles indicate measured data and the solid line a lorentzian fit $\left(825^{\circ} \mathrm{C}\right)$. 


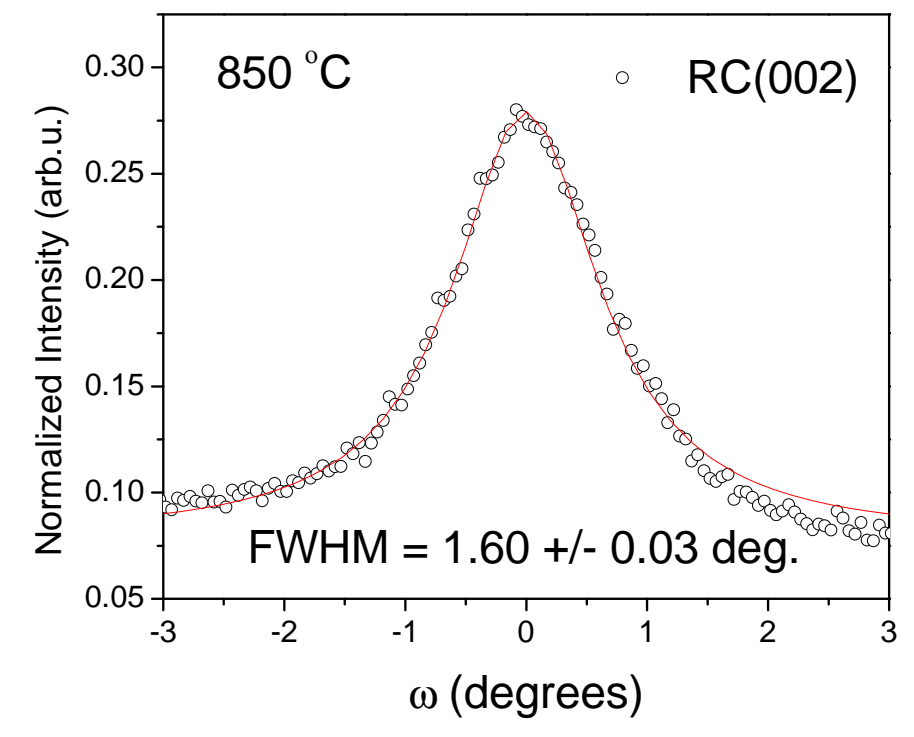

Figure 81: Rocking curve of YMO (002) reflection. Circles indicate measured data and the solid line a lorentzian fit $\left(850{ }^{\circ} \mathrm{C}\right)$.

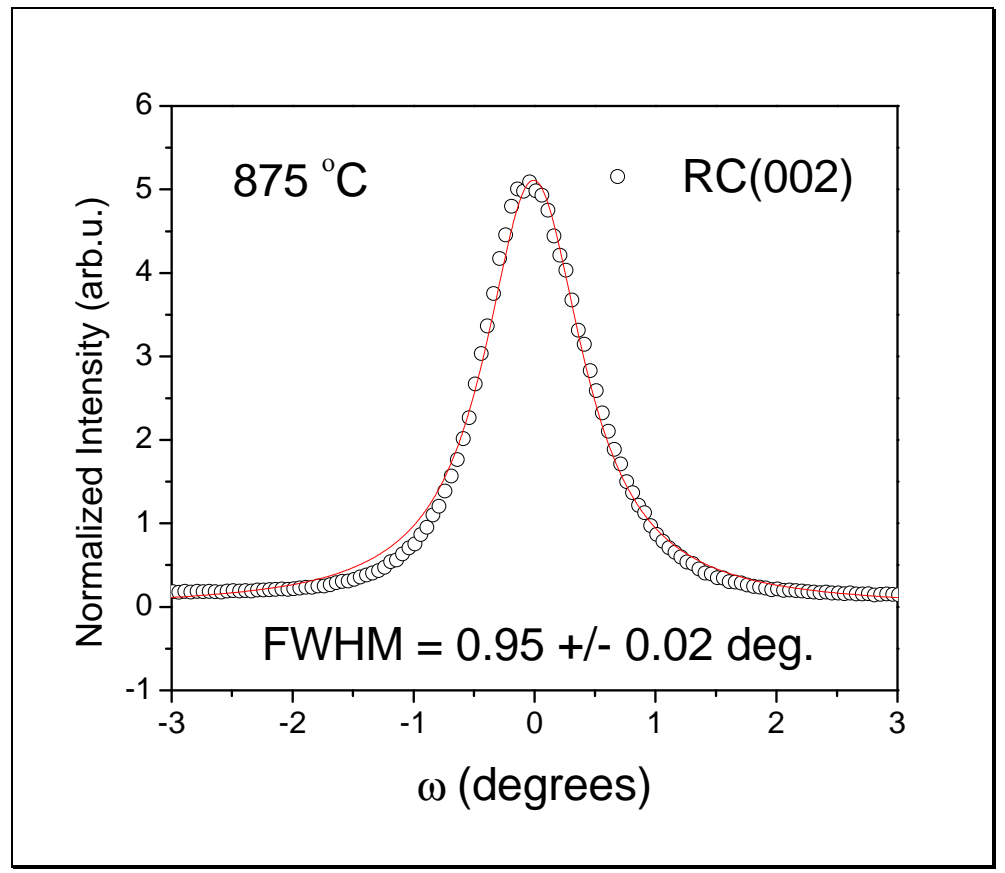

Figure 82: Rocking curve of YMO (002) reflection. Circles indicate measured data and the solid line a lorentzian fit $\left(875^{\circ} \mathrm{C}\right)$. 


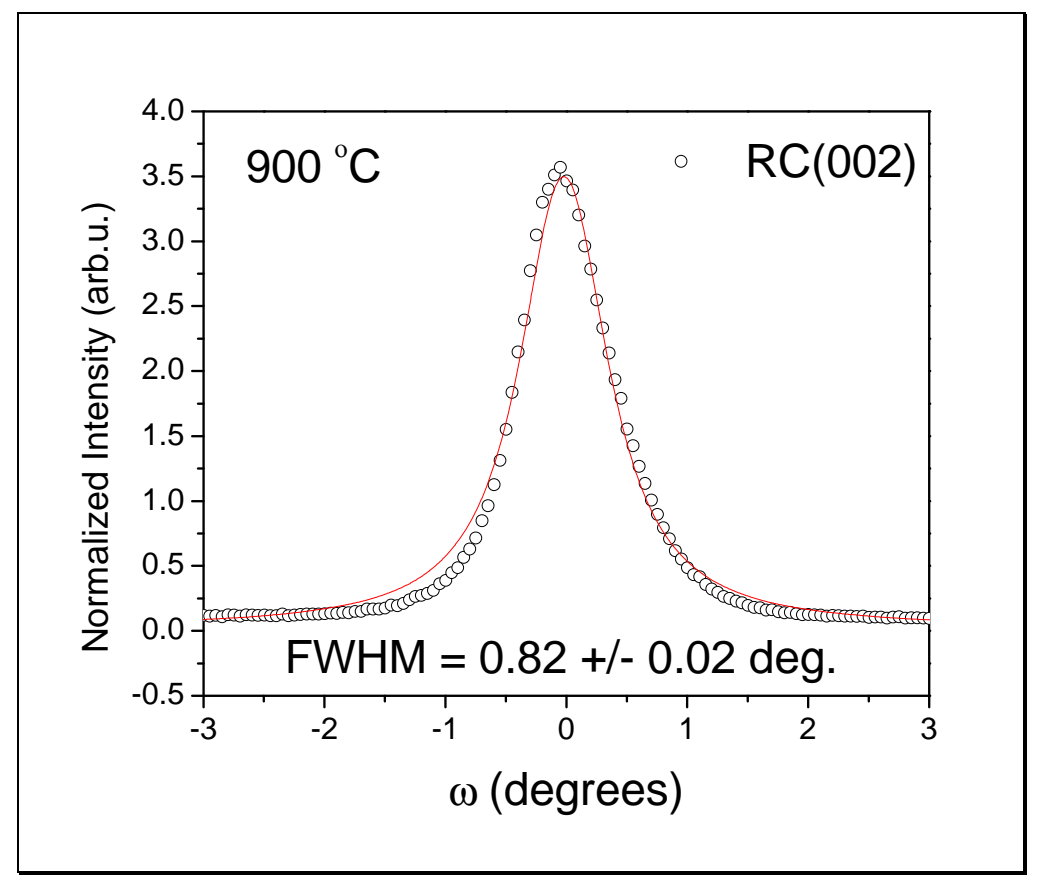

Figure 83: Rocking curve of YMO (002) reflection. Circles indicate measured data and the solid line a lorentzian fit $\left(900^{\circ} \mathrm{C}\right)$. 


\section{A.6 In-Plane-X-ray Diffraction}

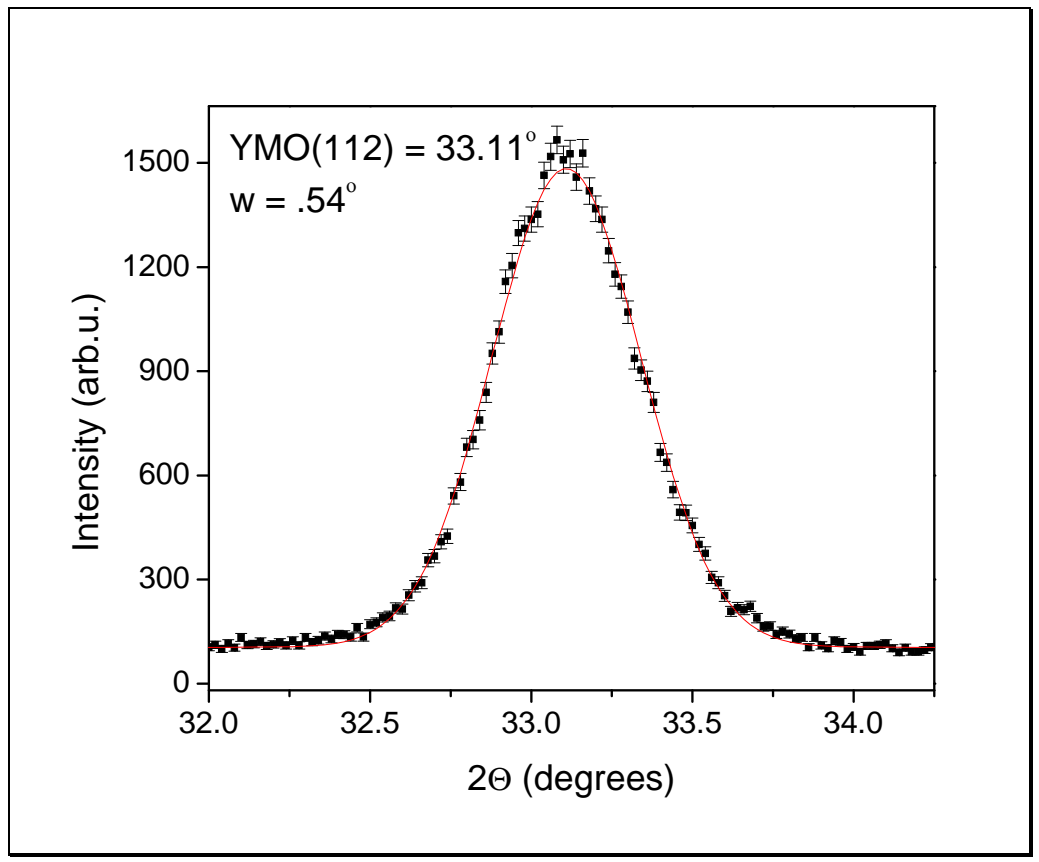

Figure 84: Gaussian fit of the YMO (112) reflection. Scatter markers denote the measured data along with the associated error. The solid line indicates the fit $\left(800{ }^{\circ} \mathrm{C}\right)$.

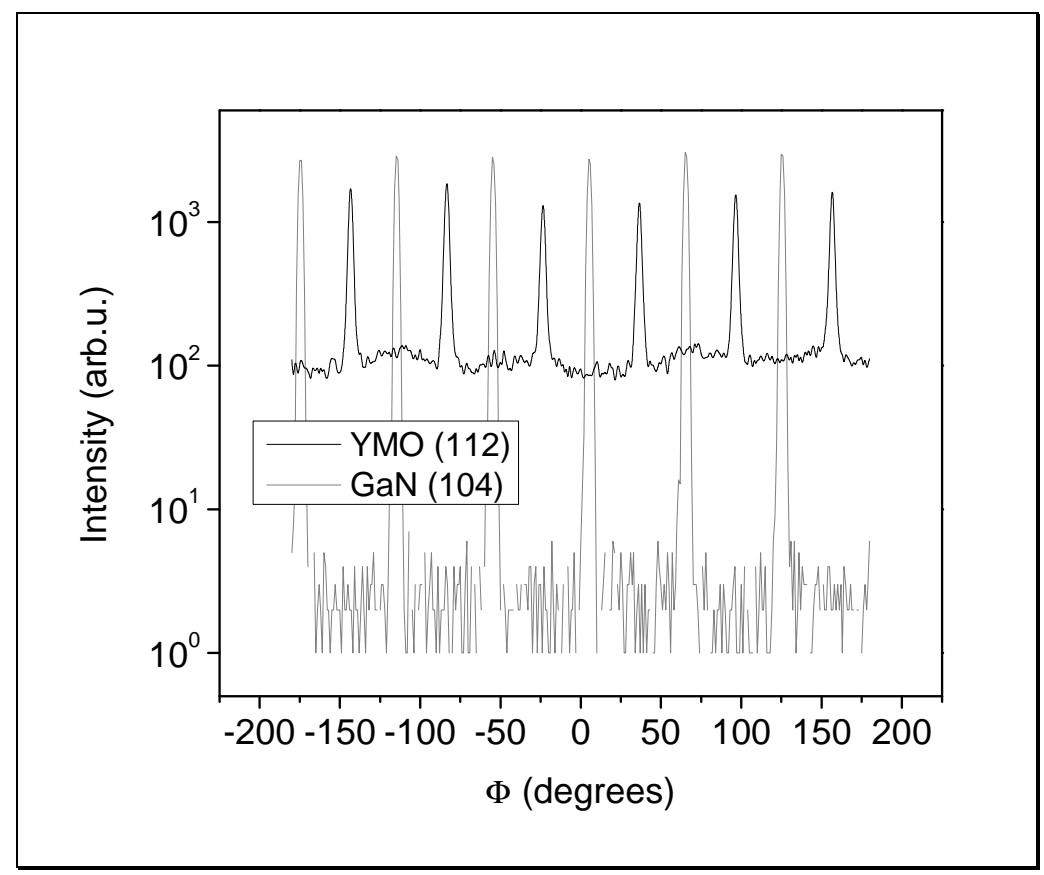

Figure 85: X-ray $\Phi$ scan indicating the in-plane epitaxial relation of YMO [1-10] || GaN [110] $\left(800 \mathrm{C}^{\circ}\right)$. 


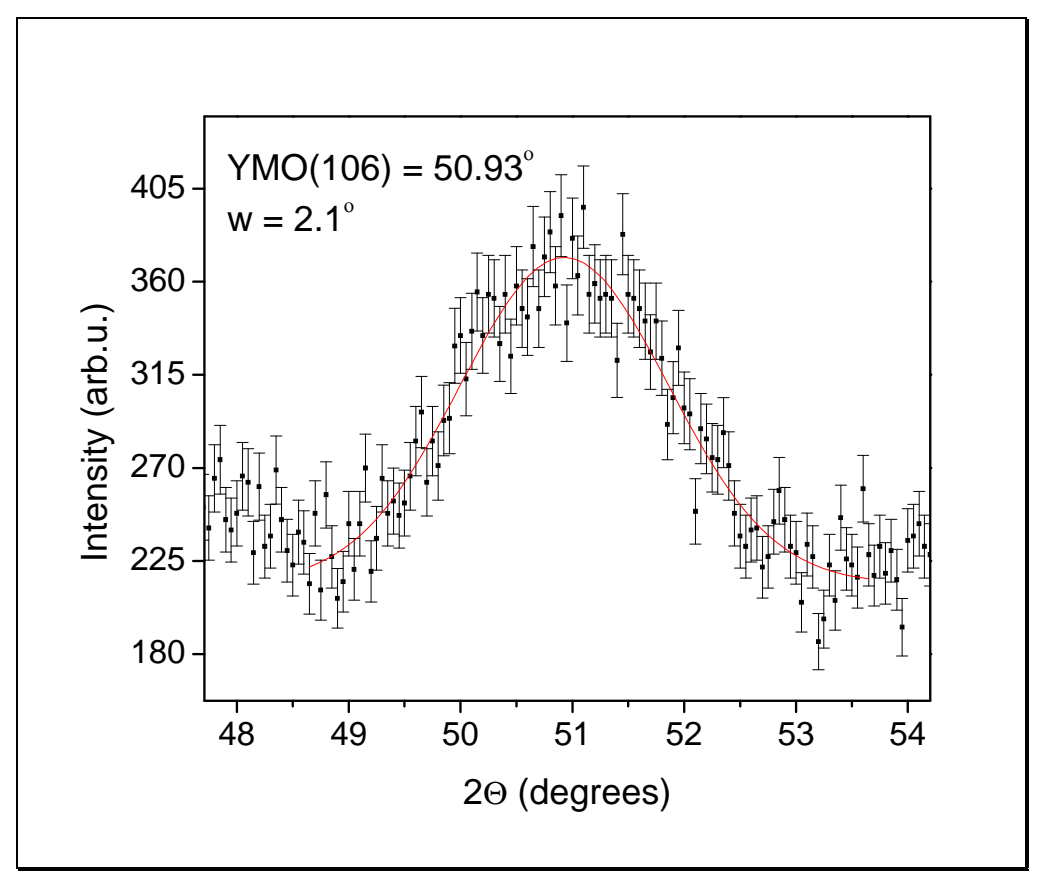

Figure 86: Gaussian fit of the YMO (106) reflection. Scatter markers denote the measured data along with the associated error. The solid line indicates the fit $\left(825^{\circ} \mathrm{C}\right)$.

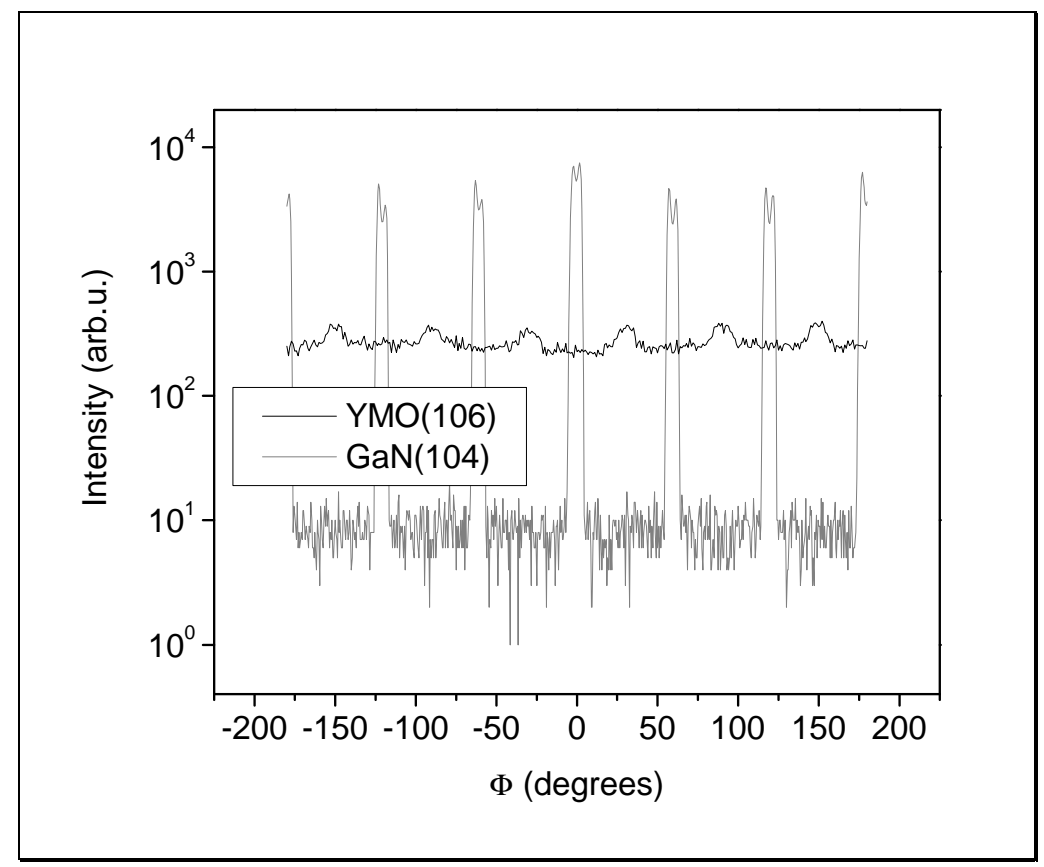

Figure 87: X-ray $\Phi$ scan indicating the in-plane epitaxial relation of YMO [1-10] || GaN [110] $\left(825 \mathrm{C}^{\circ}\right)$. 


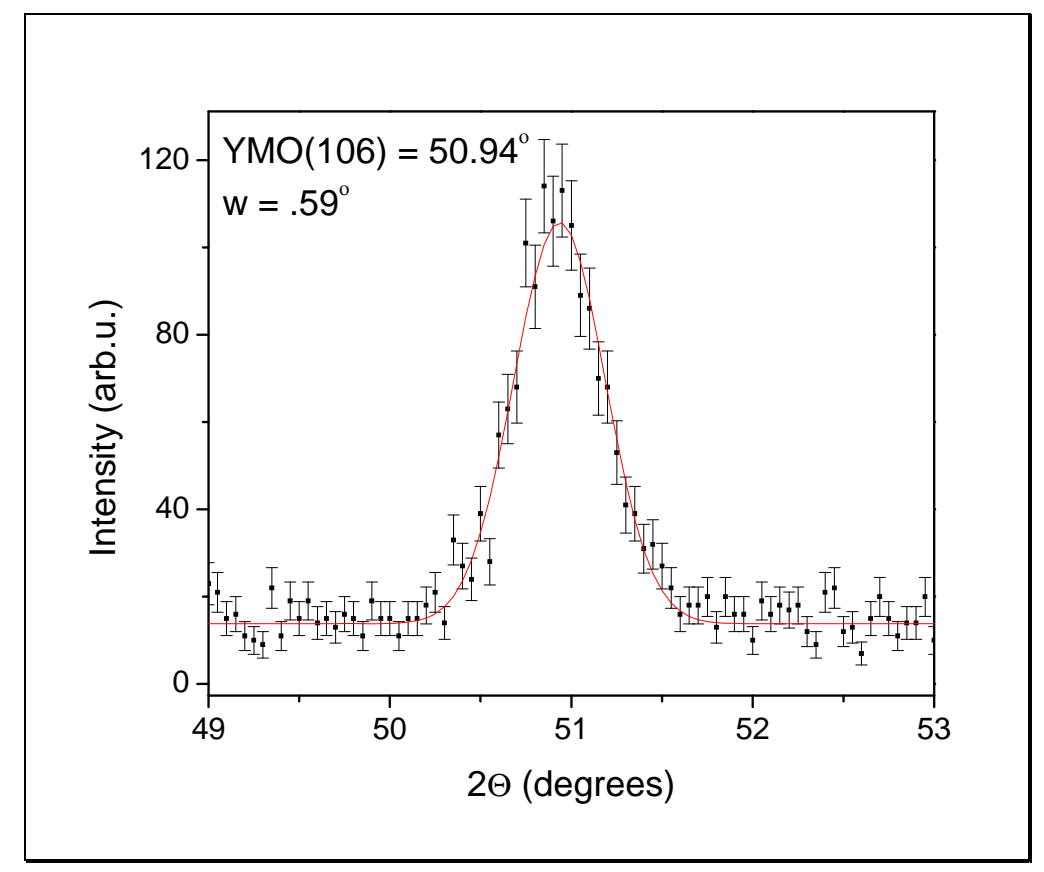

Figure 88: Gaussian fit of the YMO (106) reflection. Scatter markers denote the measured data along with the associated error. The solid line indicates the fit $\left(850{ }^{\circ} \mathrm{C}\right)$.

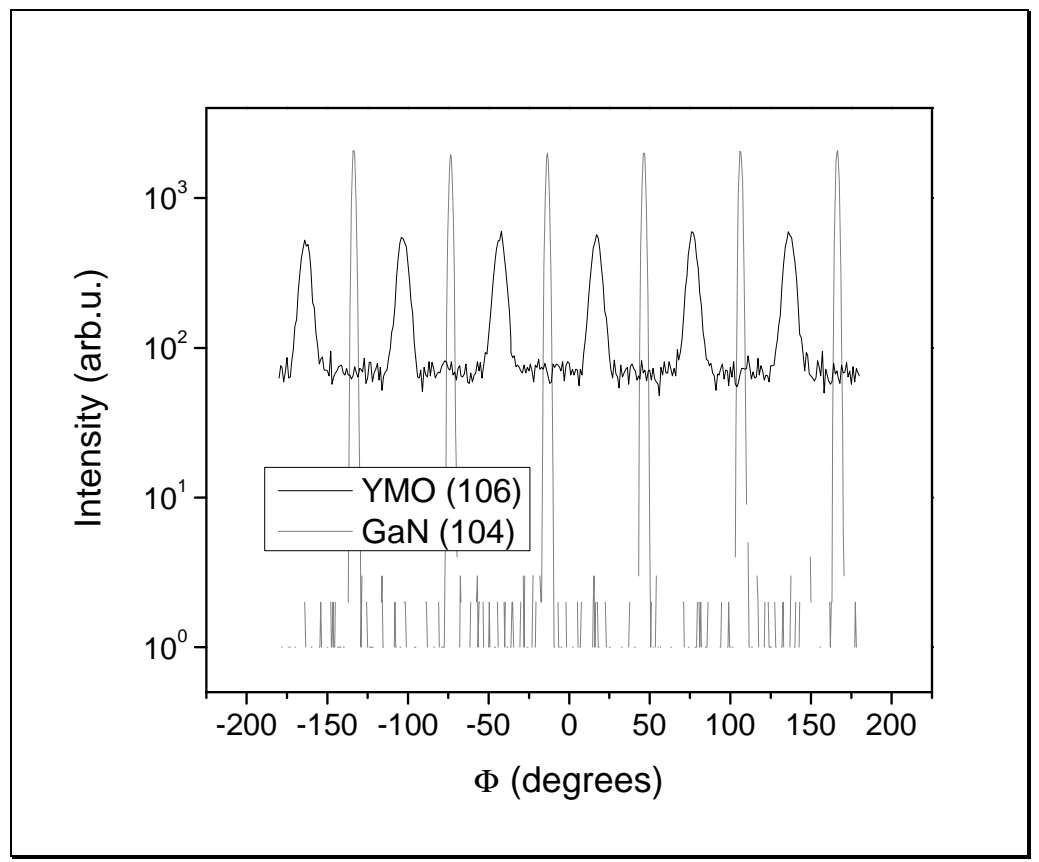

Figure 89: X-ray $\Phi$ scan indicating the in-plane epitaxial relation of YMO [1-10] || GaN [110] $\left(850{ }^{\circ} \mathrm{C}\right)$. 


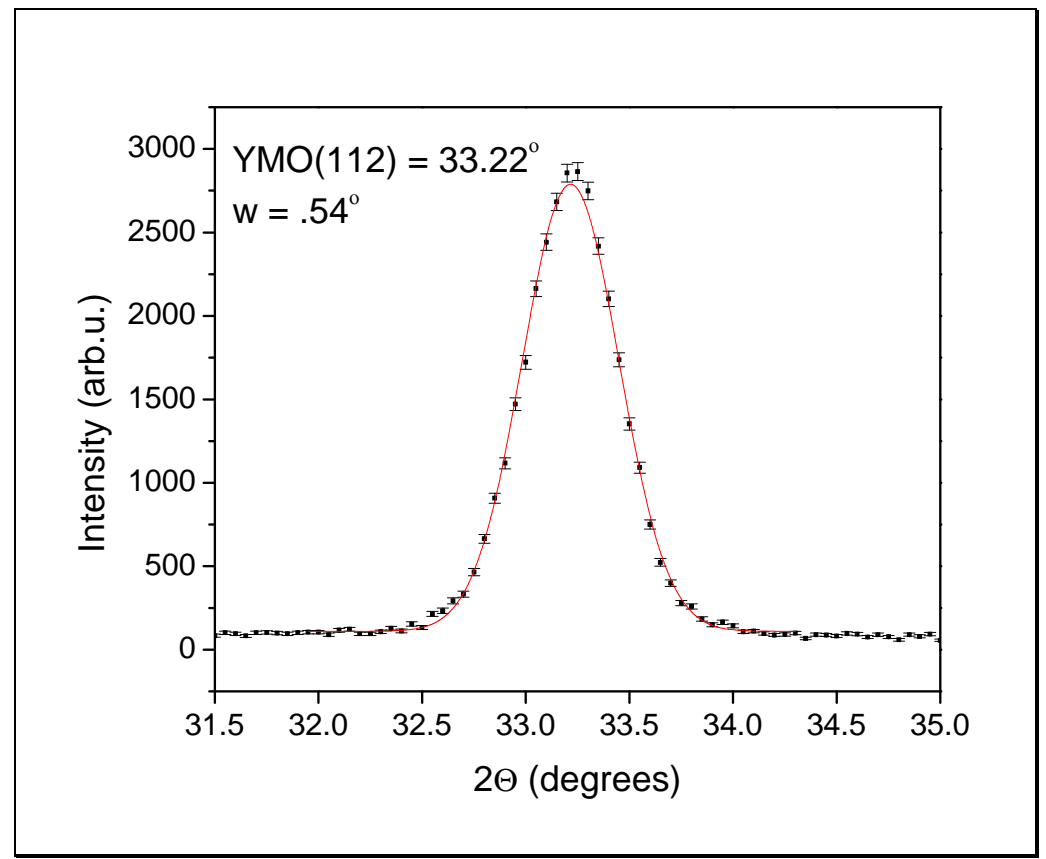

Figure 90: Gaussian fit of the YMO (112) reflection. Scatter markers denote the measured data along with the associated error. The solid line indicates the fit $\left(875^{\circ} \mathrm{C}\right)$.

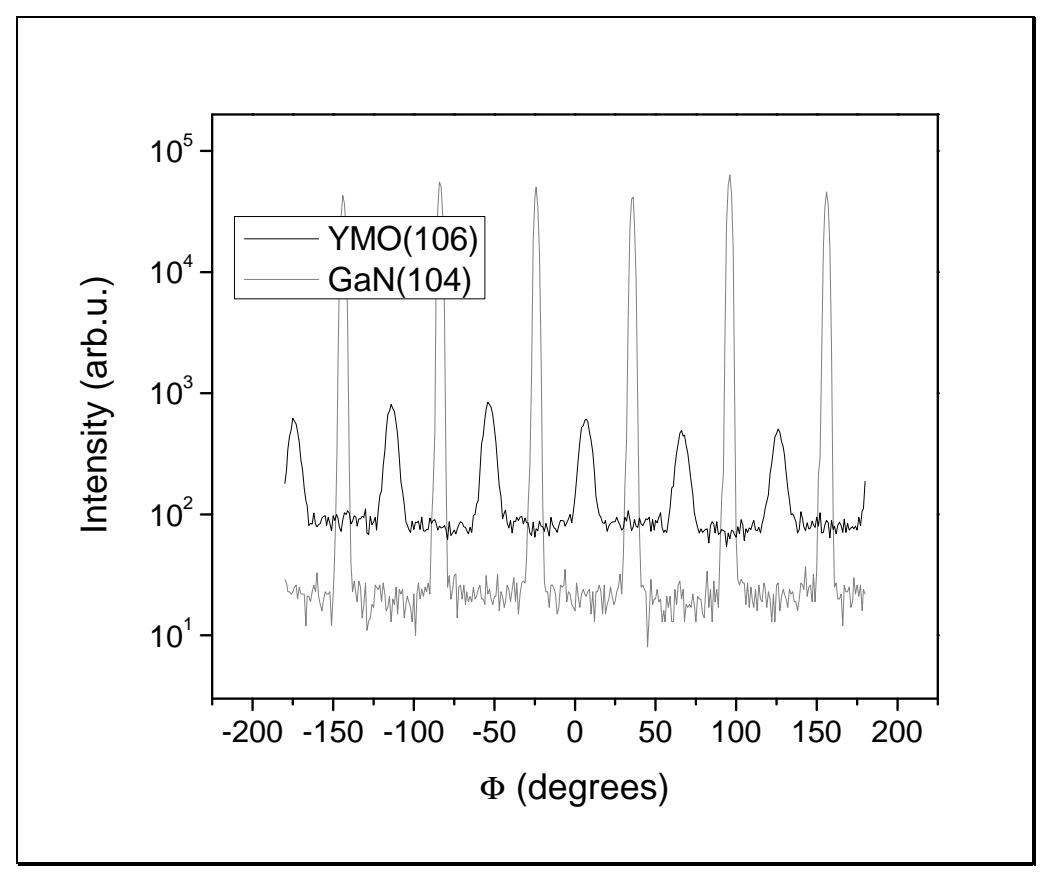

Figure 91: X-ray $\Phi$ scan indicating the in-plane epitaxial relation of YMO [1-10] || GaN [110] $\left(875^{\circ} \mathrm{C}\right)$. 


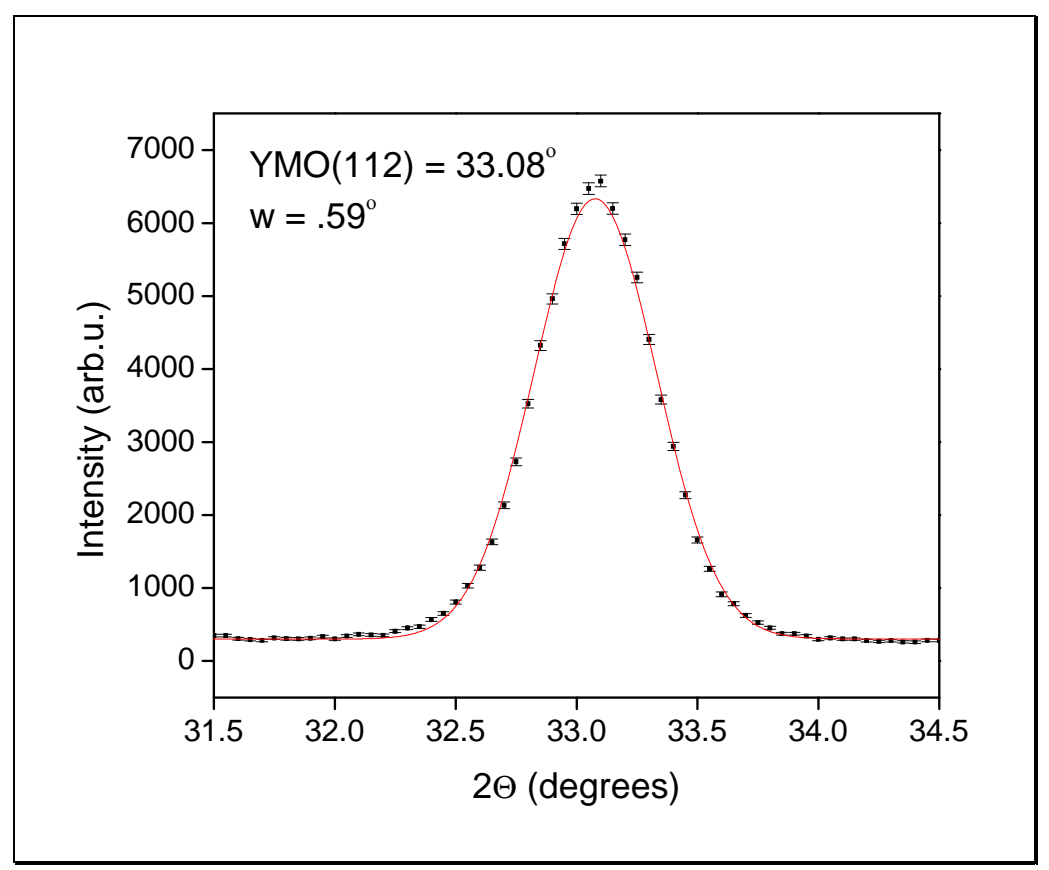

Figure 92: Gaussian fit of the YMO (112) reflection. Scatter markers denote the measured data along with the associated error. The solid line indicates the fit $\left(900{ }^{\circ} \mathrm{C}\right)$.

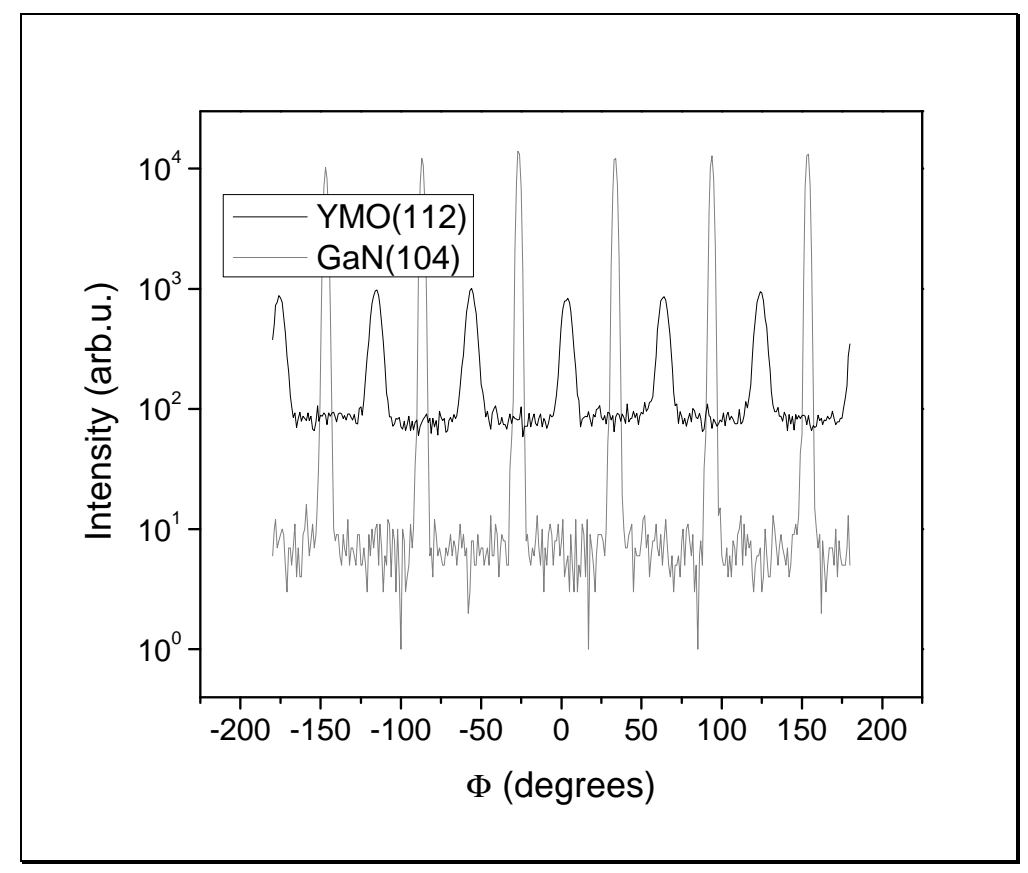

Figure 93: X-ray $\Phi$ scan indicating the in-plane epitaxial relation of YMO [1-10] || GaN [110] $\left(900{ }^{\circ} \mathrm{C}\right)$. 


\section{A.7 X-Ray Reflectivity}

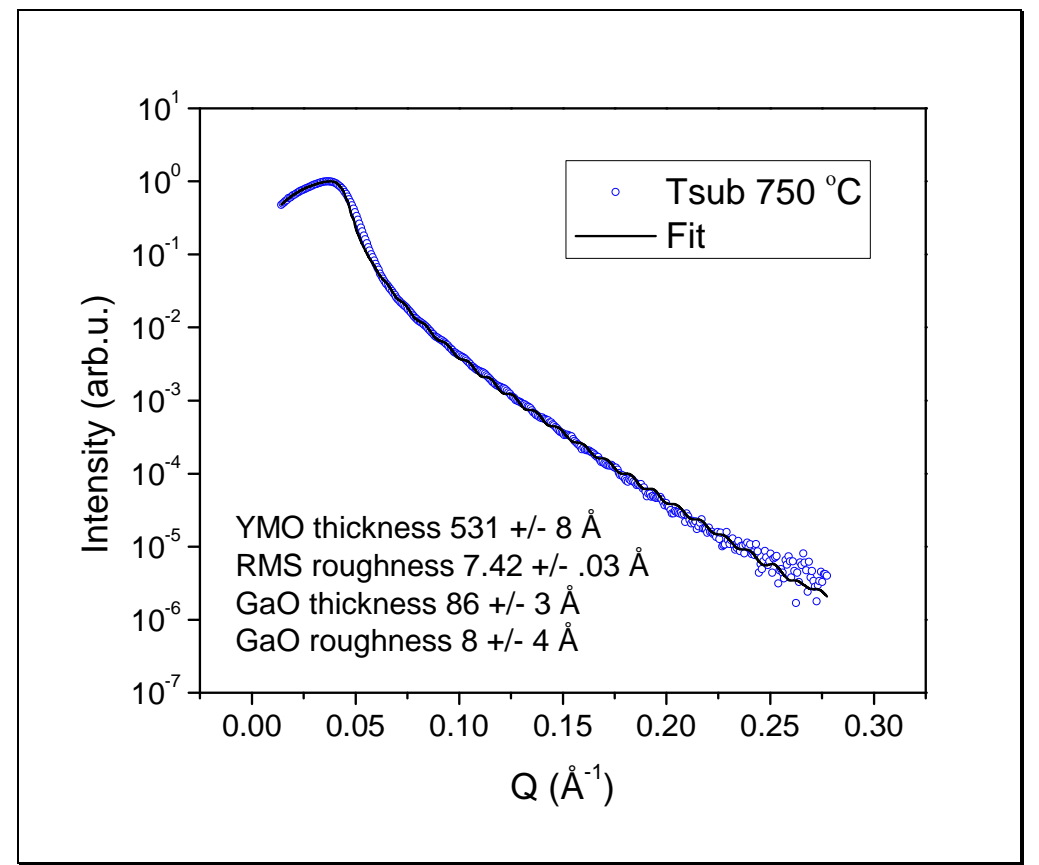

Figure 94: $\mathrm{X}$-ray reflectivity of sample grown at $750{ }^{\circ} \mathrm{C}$.

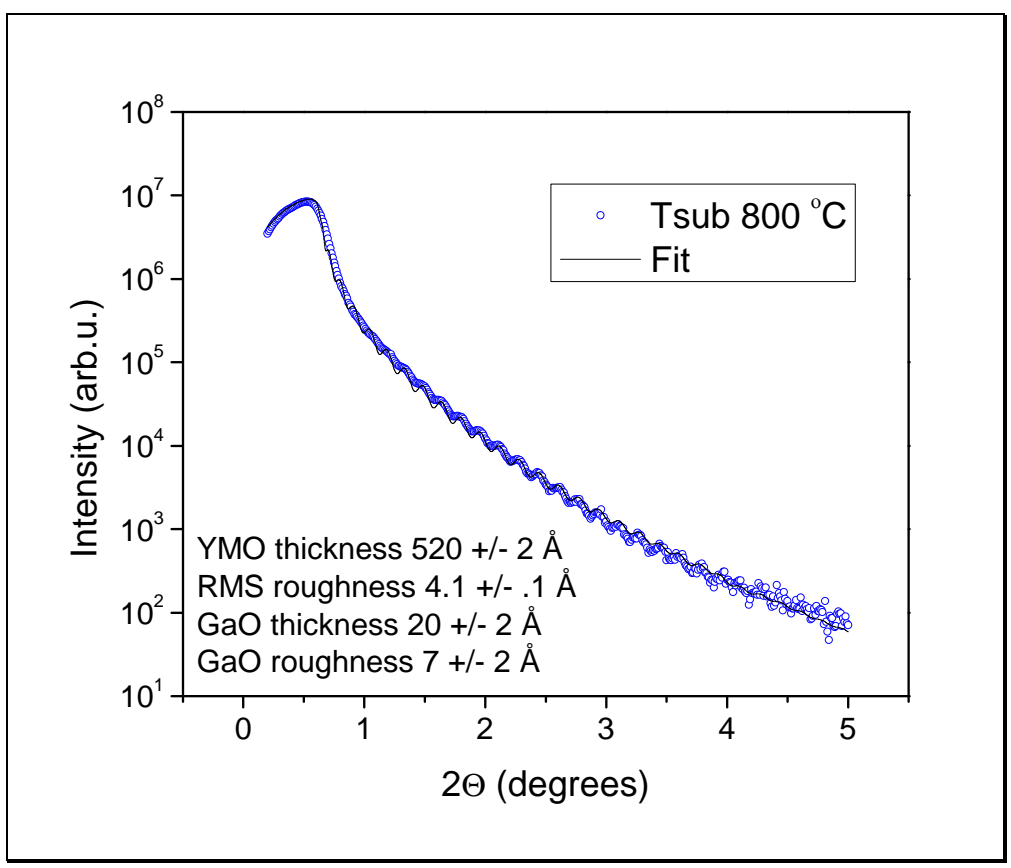

Figure 95: X-ray reflectivity of sample grown at $800{ }^{\circ} \mathrm{C}$. 


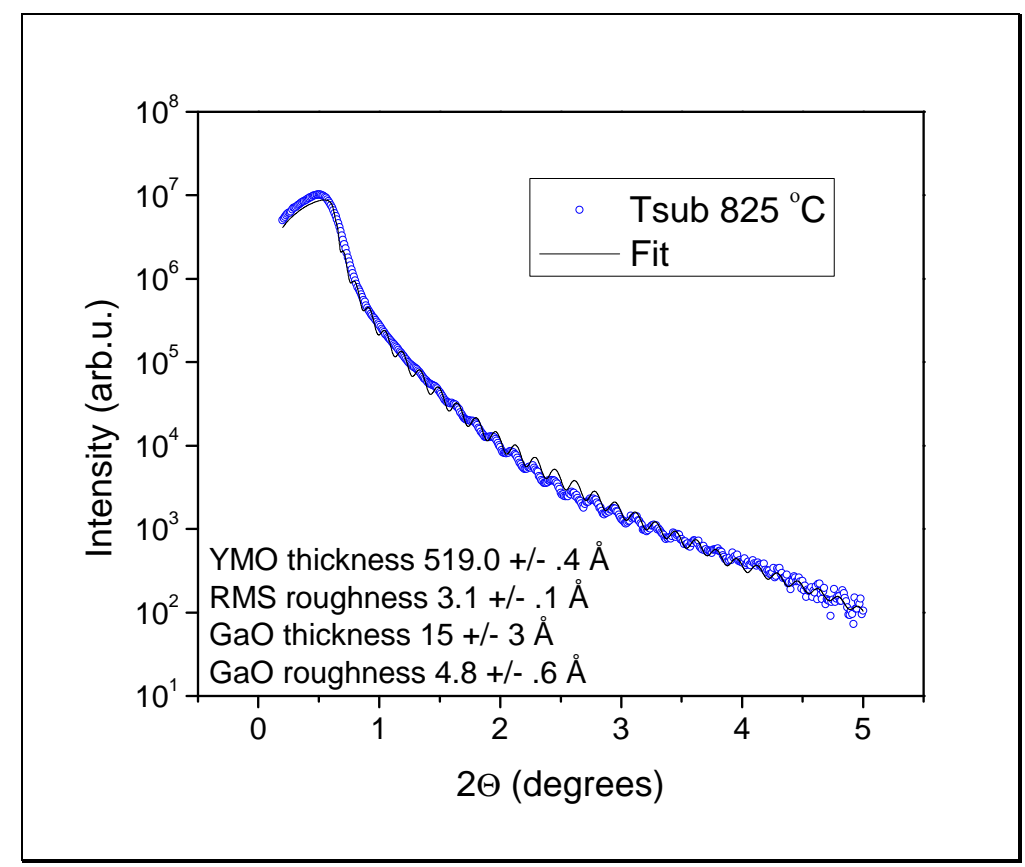

Figure 96: X-ray reflectivity of sample grown at $825^{\circ} \mathrm{C}$.

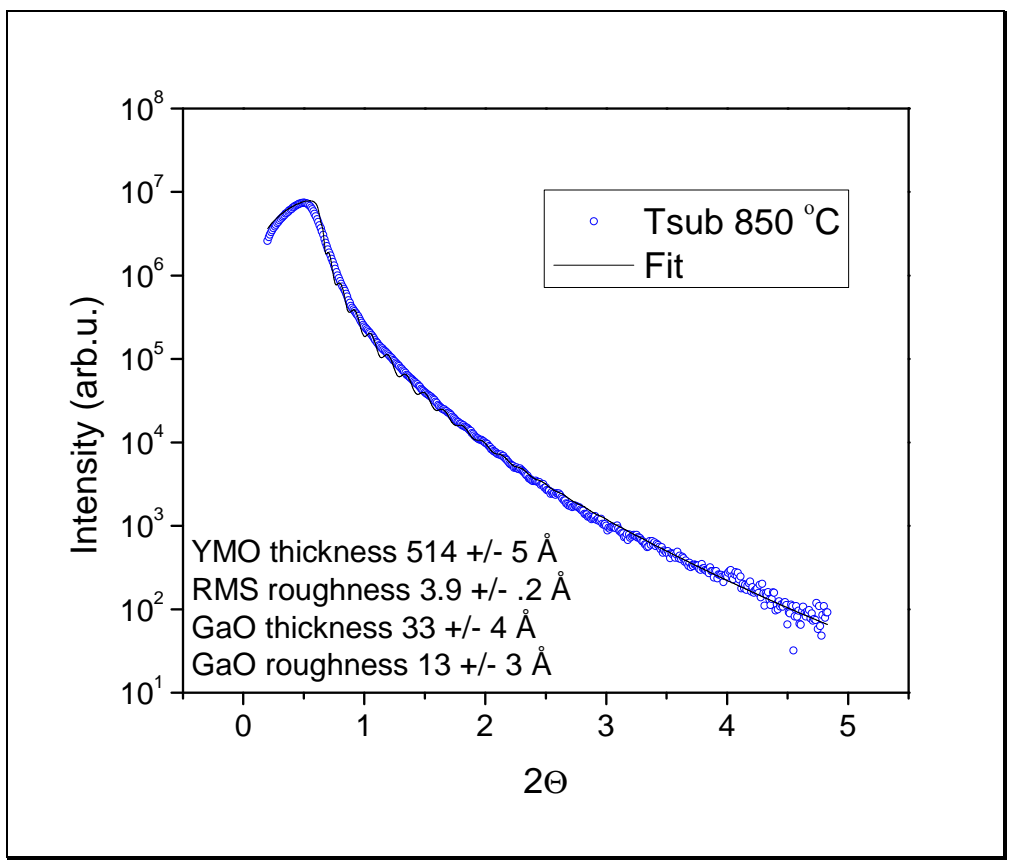

Figure 97: X-ray reflectivity of sample grown at $850{ }^{\circ} \mathrm{C}$. 


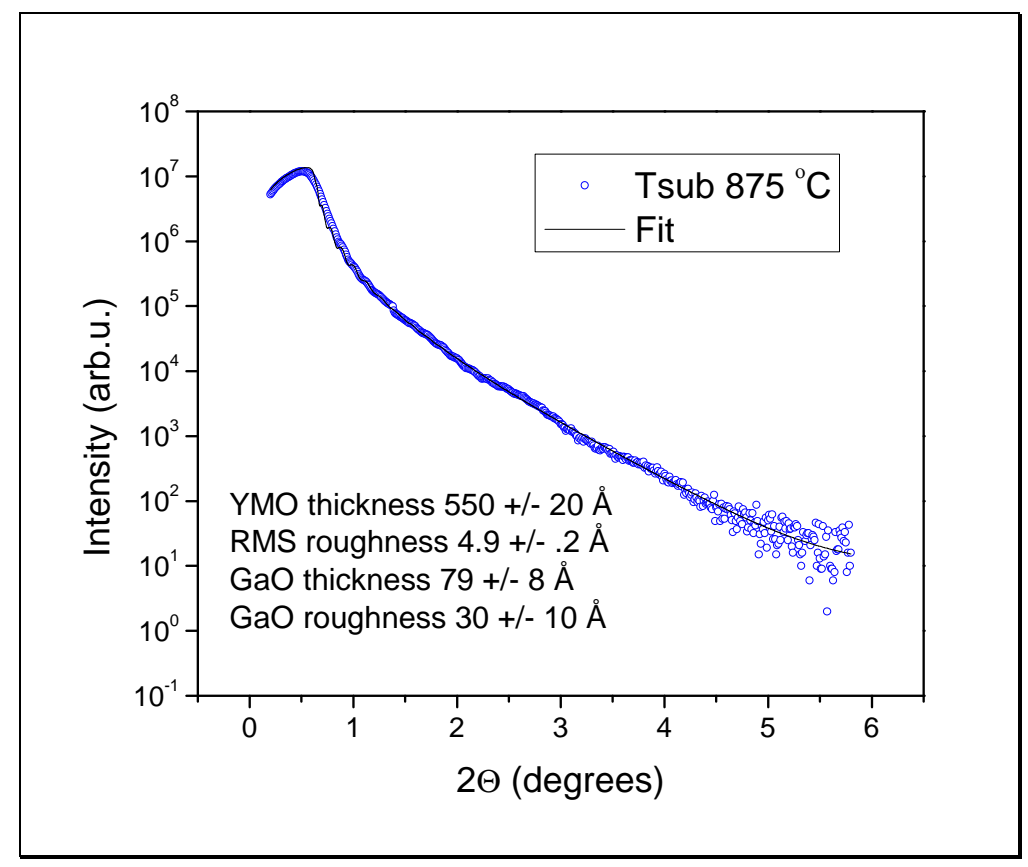

Figure 98: X-ray reflectivity of sample grown at $875^{\circ} \mathrm{C}$.

\section{A.8 Electrical Characterization}

\section{A.8.1 $\quad T_{\text {sub }}=800 \mathrm{C}$}
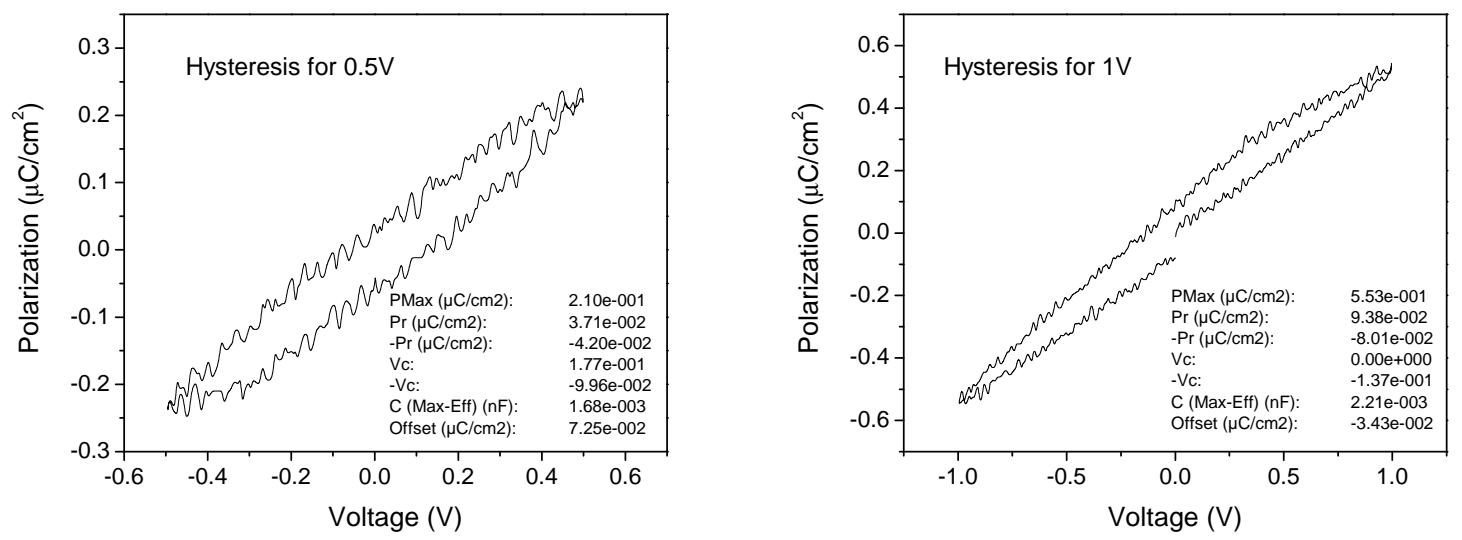

Figure 99: $P-V$ loops for $0.5 \mathrm{~V}$ and $1 \mathrm{~V}\left(800{ }^{\circ} \mathrm{C}\right)$. 


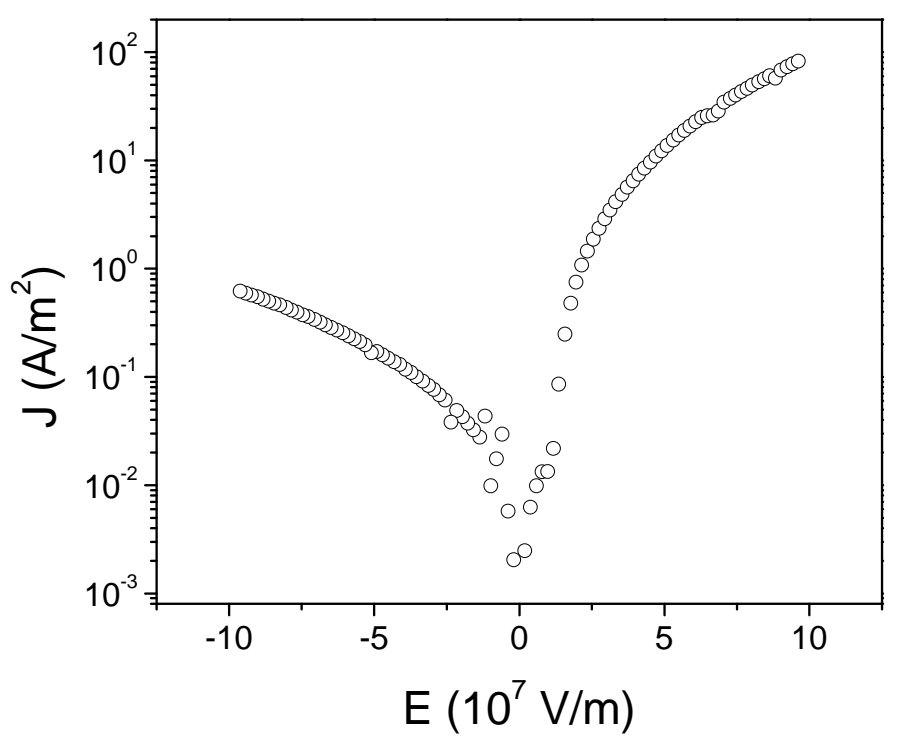

Figure 100: $J$ vs. $E$ for $800{ }^{\circ} \mathrm{C}$.

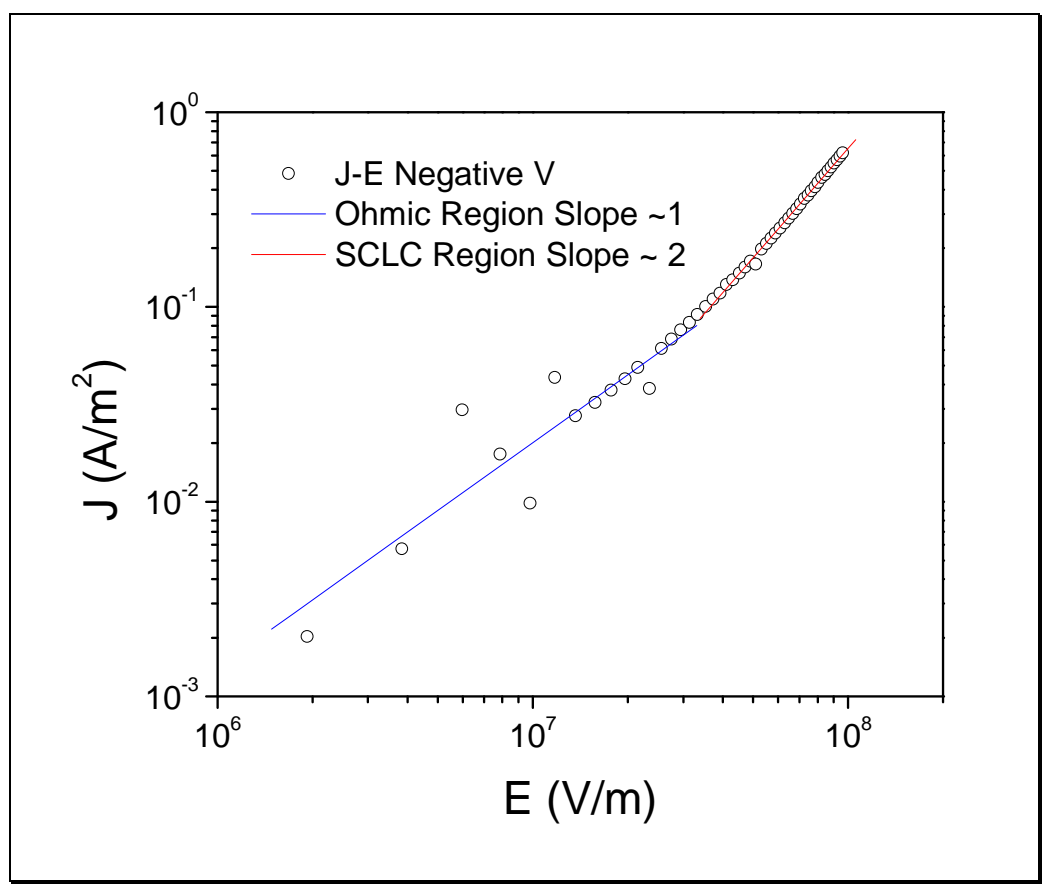

Figure 101: $\log J$ vs. $\log E$ for negative voltage $\left(800{ }^{\circ} \mathrm{C}\right)$. Each solid, colored line represents a linear fit to a separate leakage region. 


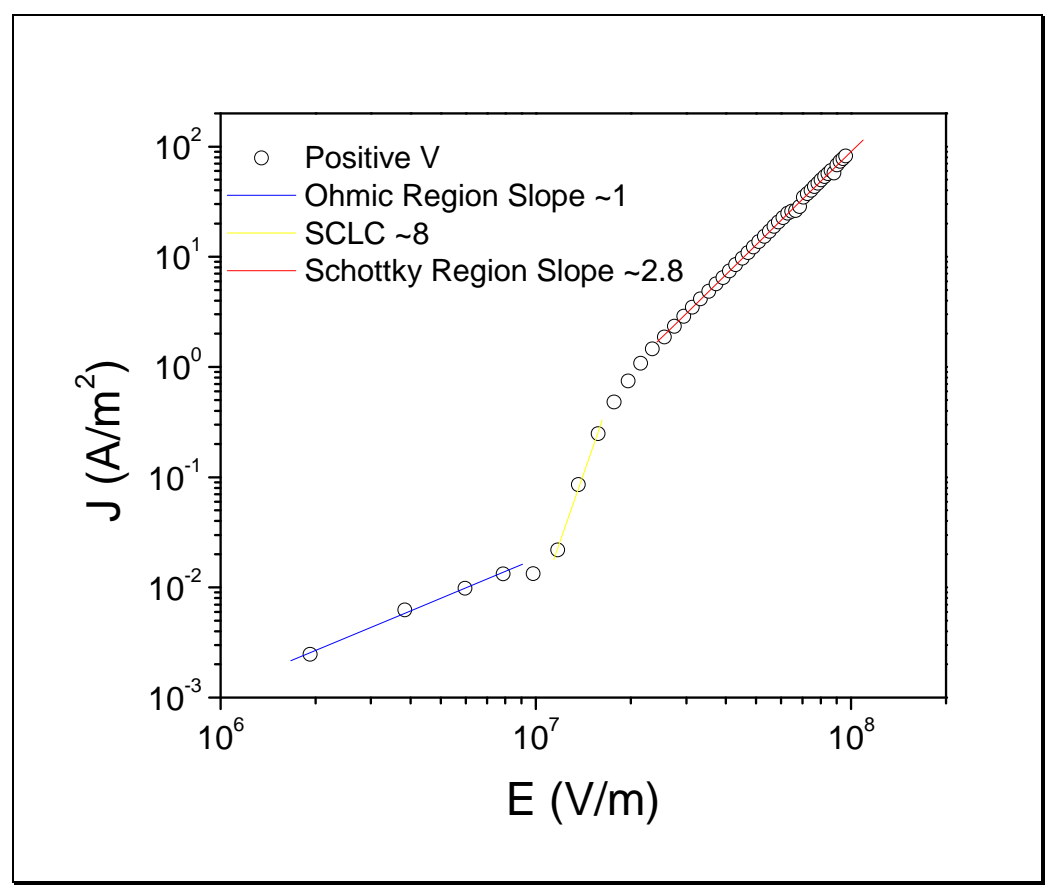

Figure 102: $\log J$ vs. $\log E$ for positive voltage $\left(800{ }^{\circ} \mathrm{C}\right)$. Each solid, colored line represents a linear fit to a separate leakage region.

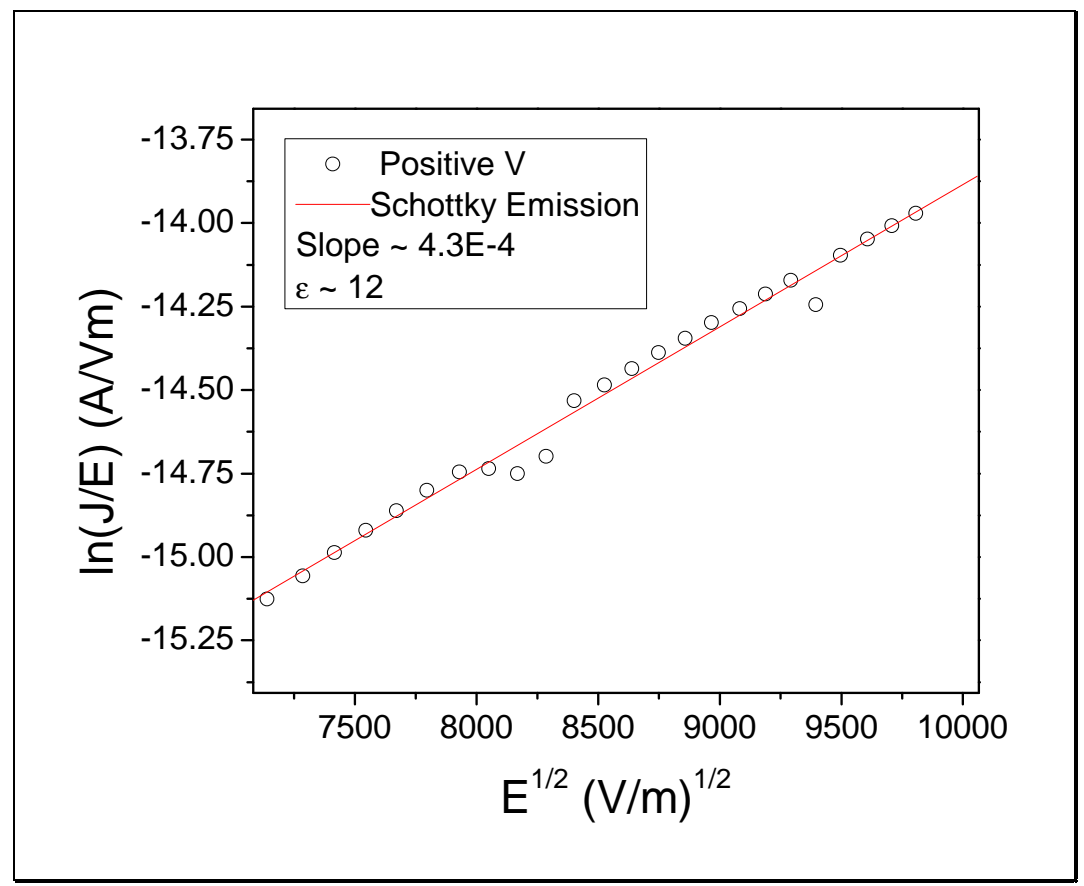

Figure 103: $\operatorname{Ln}(J / E)$ vs. $\mathrm{E}^{1 / 2}$ for positive voltage $\left(800^{\circ} \mathrm{C}\right)$. The line is the linear fit to region corresponding to Schottky emission. 


\section{A.8.2 $\quad T_{\text {sub }}=825 \mathrm{C}$}
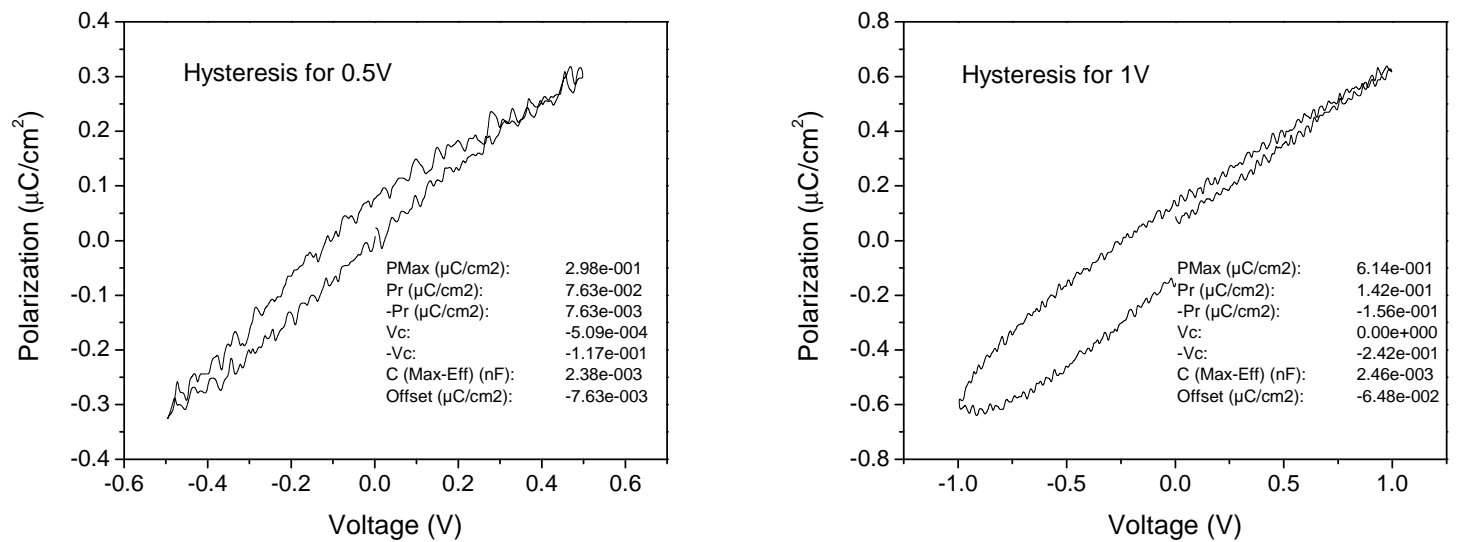

Figure 104: $P-V$ loops for $0.5 \mathrm{~V}$ and $1 \mathrm{~V}\left(825^{\circ} \mathrm{C}\right)$.

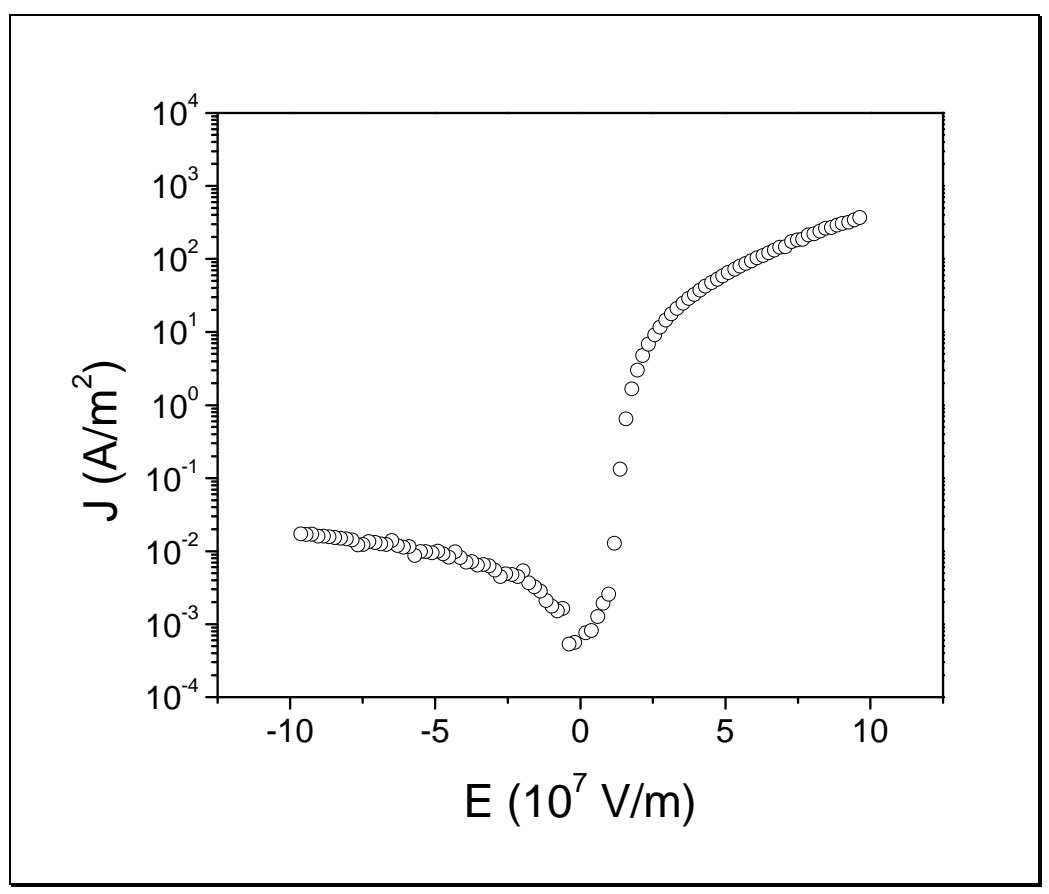

Figure 105: $J$ vs. $E$ for $825^{\circ} \mathrm{C}$. 


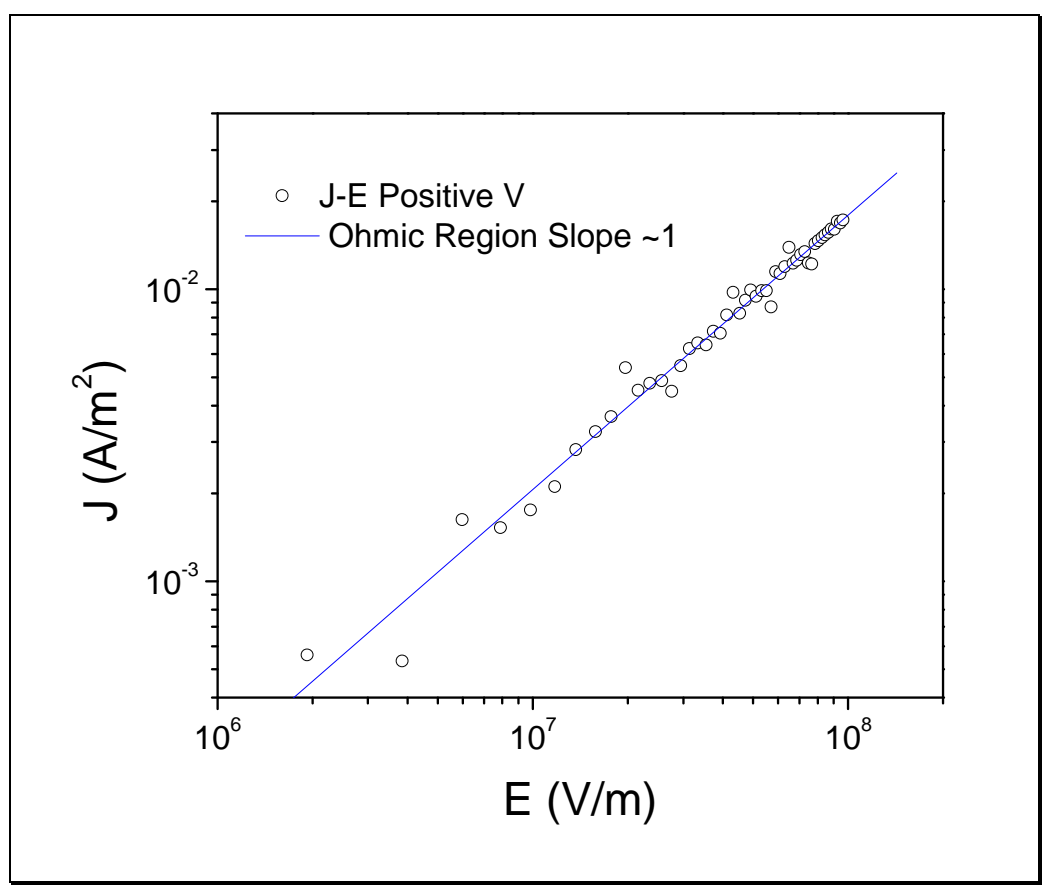

Figure 106: $\log J$ vs. $\log E$ for negative voltage $\left(825^{\circ} \mathrm{C}\right)$. Slope is $\sim 1$ across entire range.

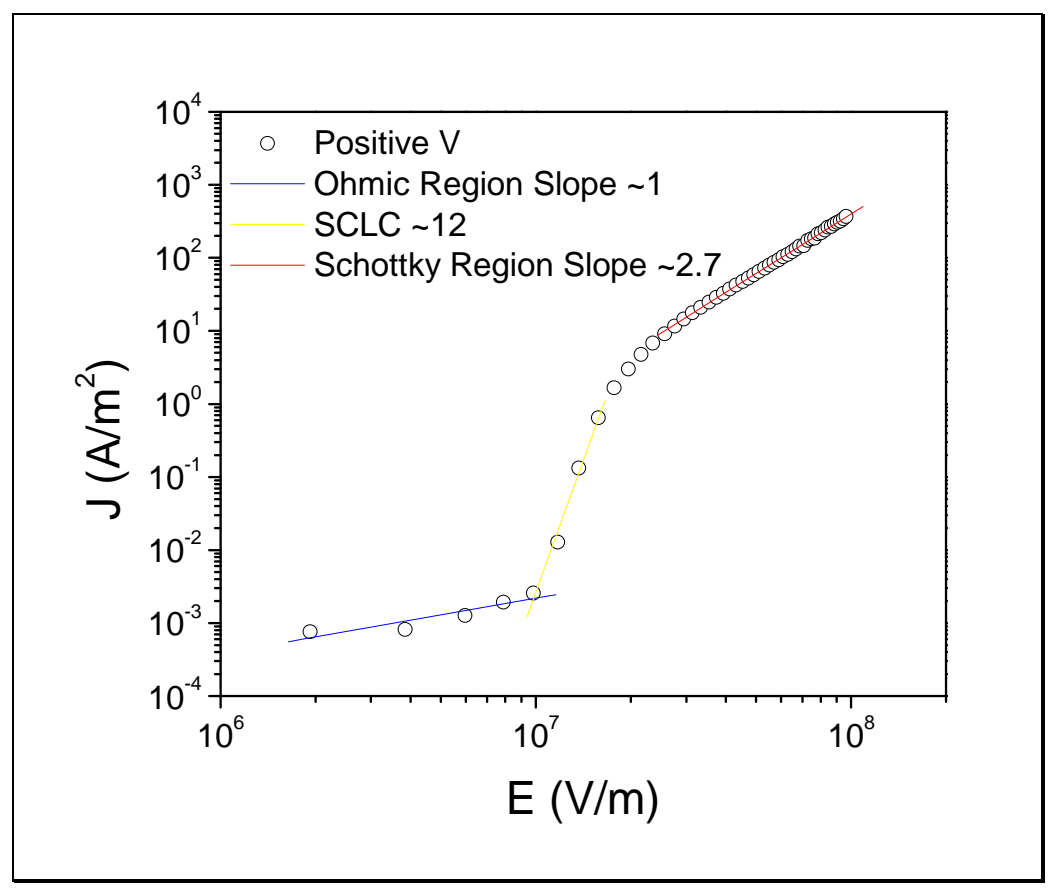

Figure 107: $\log J$ vs. $\log E$ for positive voltage $\left(825^{\circ} \mathrm{C}\right)$. Each solid, colored line represents a linear fit to a separate leakage region. 


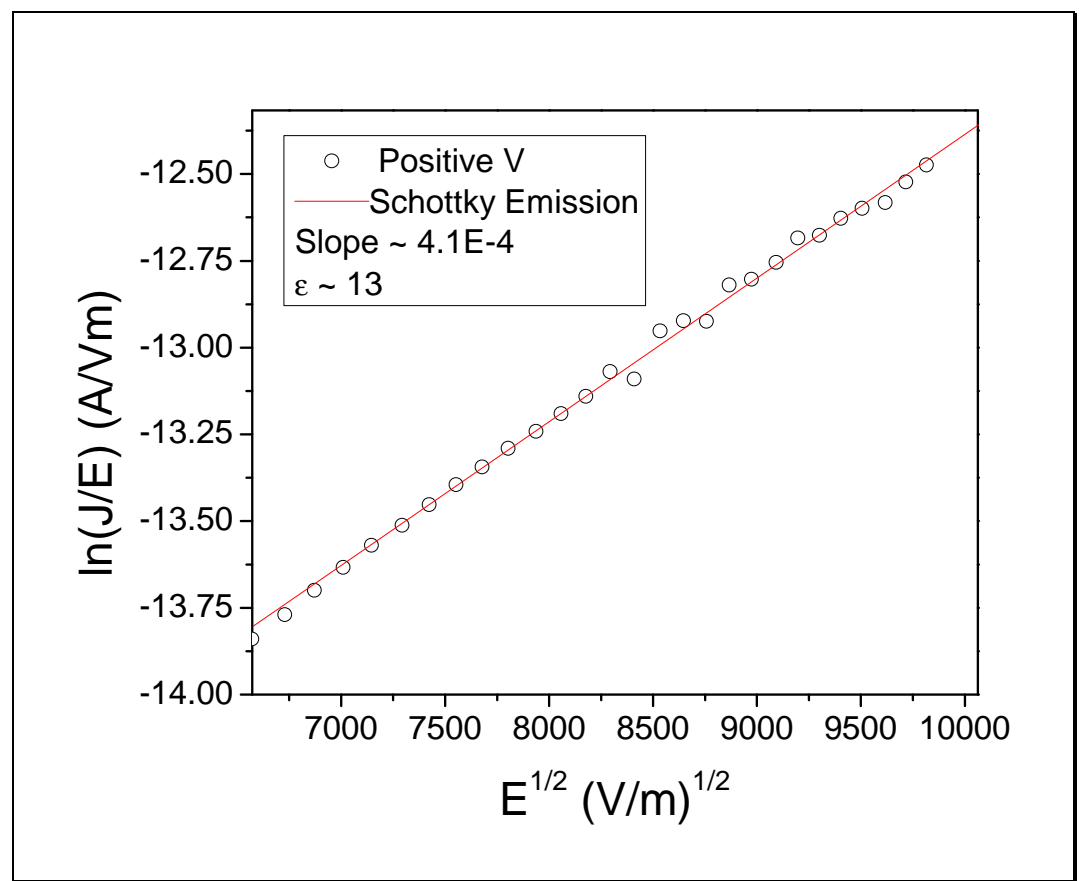

Figure 108: $\operatorname{Ln}(J / E)$ vs. $\mathrm{E}^{1 / 2}$ for positive voltage $\left(825^{\circ} \mathrm{C}\right)$. The line is the linear fit to region corresponding to Schottky emission.

\section{A.8.3 $\quad T_{\text {sub }}=850 \mathrm{C}$}
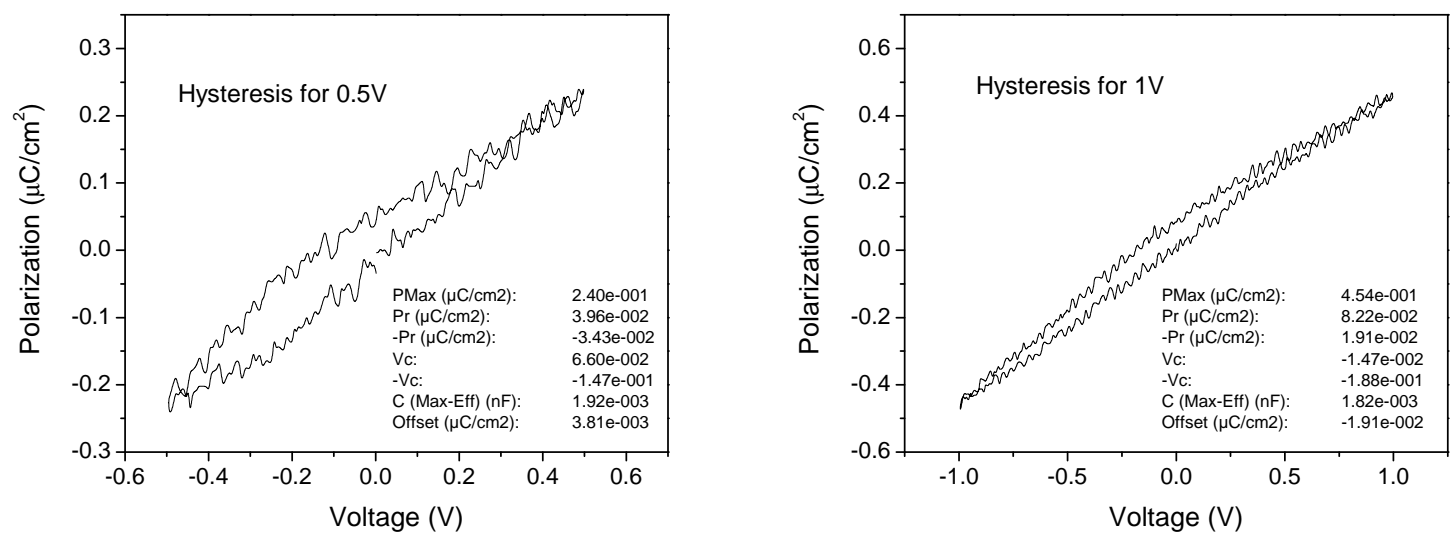

Figure 109: $P-V$ loops for $0.5 \mathrm{~V}$ and $1 \mathrm{~V}\left(850^{\circ} \mathrm{C}\right)$. 


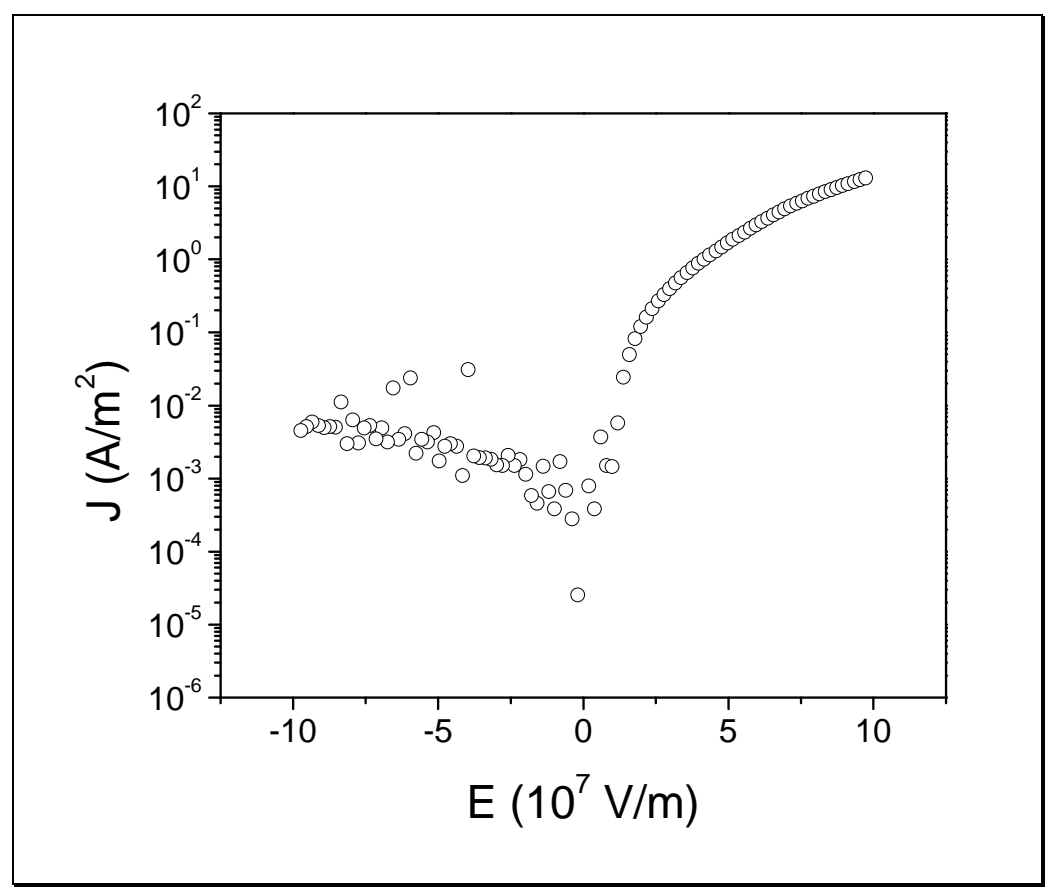

Figure 110: $J$ vs. $E$ for $850^{\circ} \mathrm{C}$.

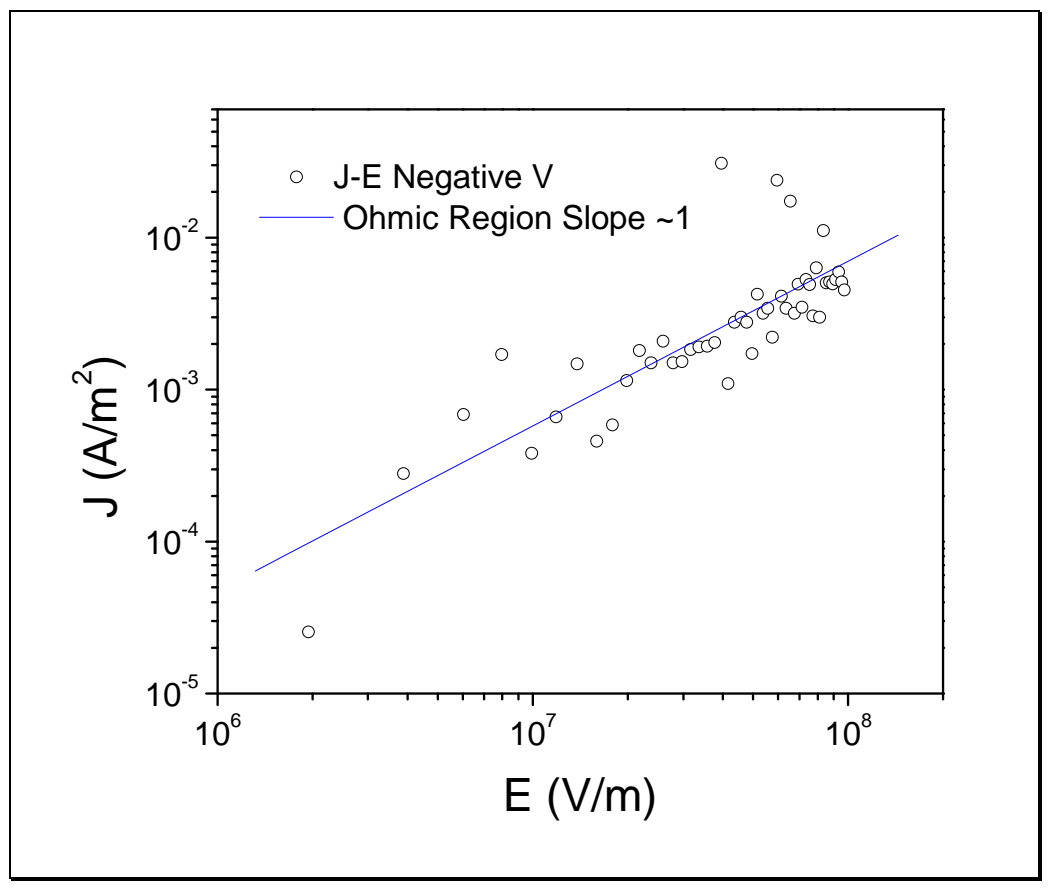

Figure 111: $\log J$ vs. $\log E$ for negative voltage $\left(850{ }^{\circ} \mathrm{C}\right)$. Slope is $\sim 1$ across entire range. 


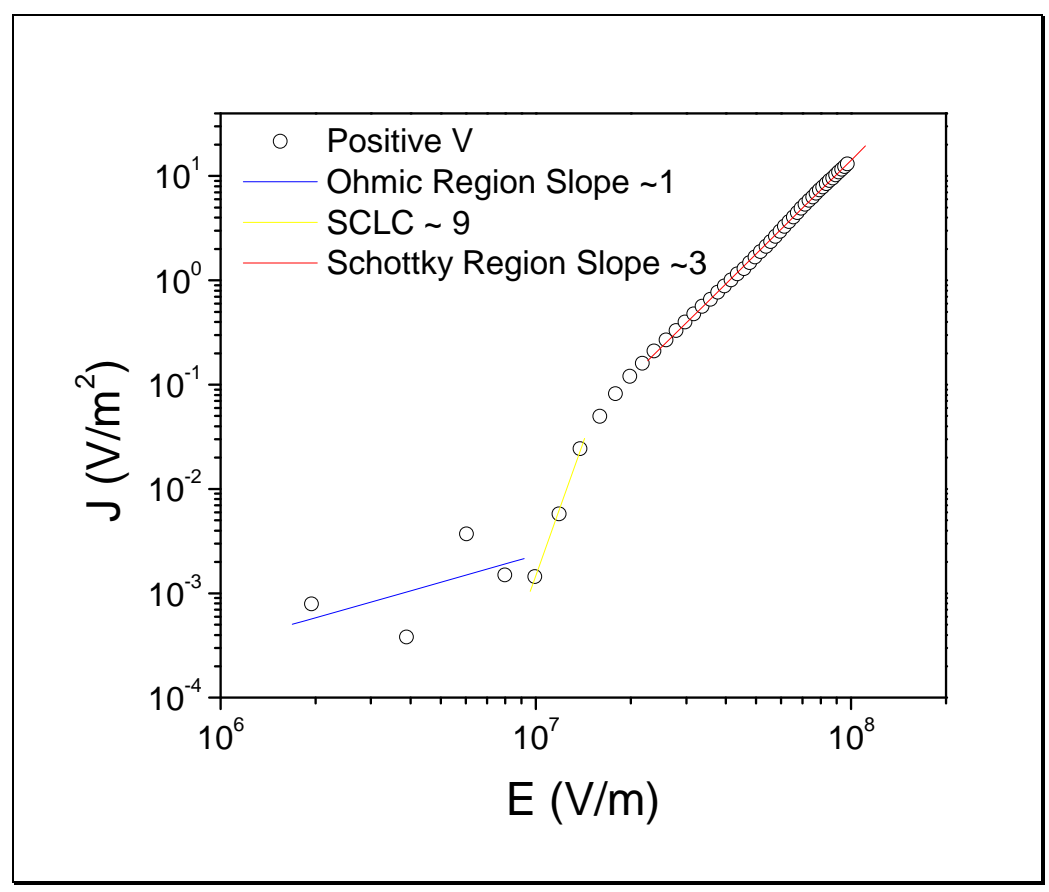

Figure 112: $\log J$ vs. $\log E$ for positive voltage $\left(850{ }^{\circ} \mathrm{C}\right)$. Each solid, colored line represents a linear fit to a separate leakage region.

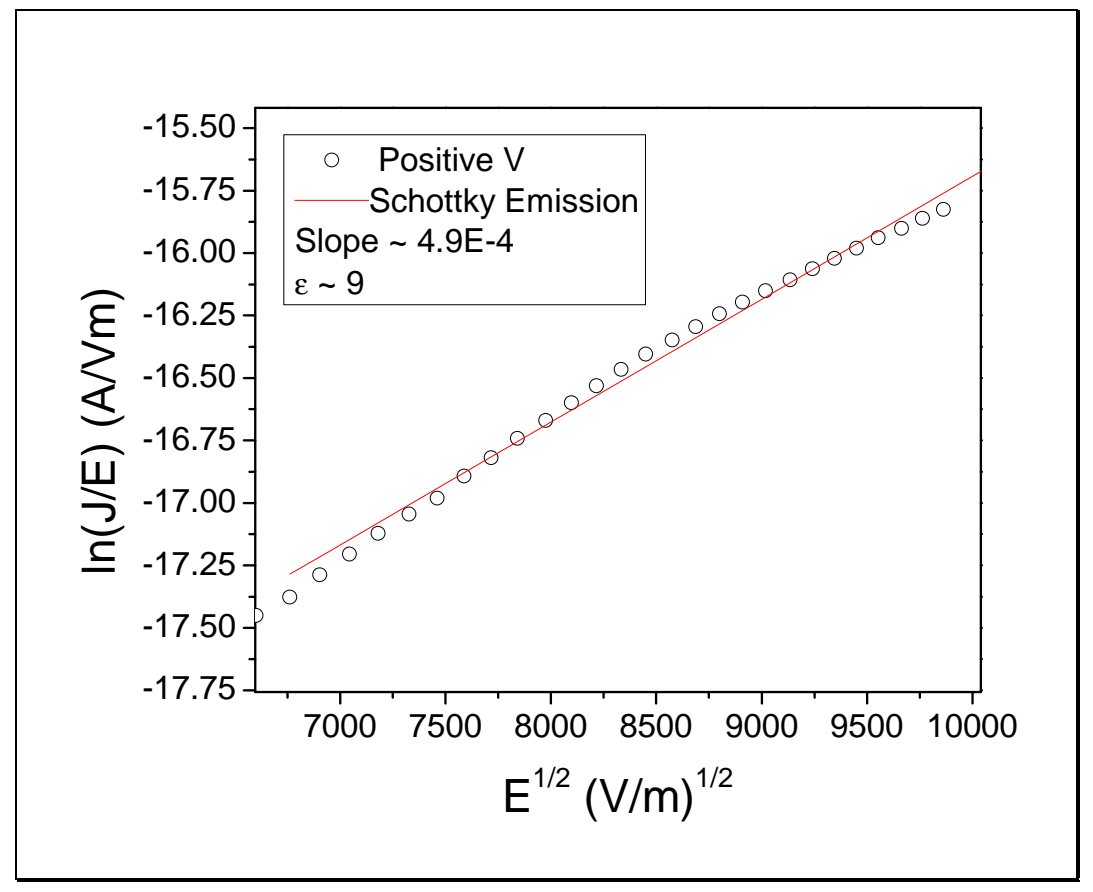

Figure 113: $\operatorname{Ln}(J / E)$ vs. $E^{1 / 2}$ for negative voltage $\left(850^{\circ} \mathrm{C}\right)$. The line is the linear fit to region corresponding to Schottky emission. 
A.8.4 $T_{\text {sub }}=875 \mathrm{C}$
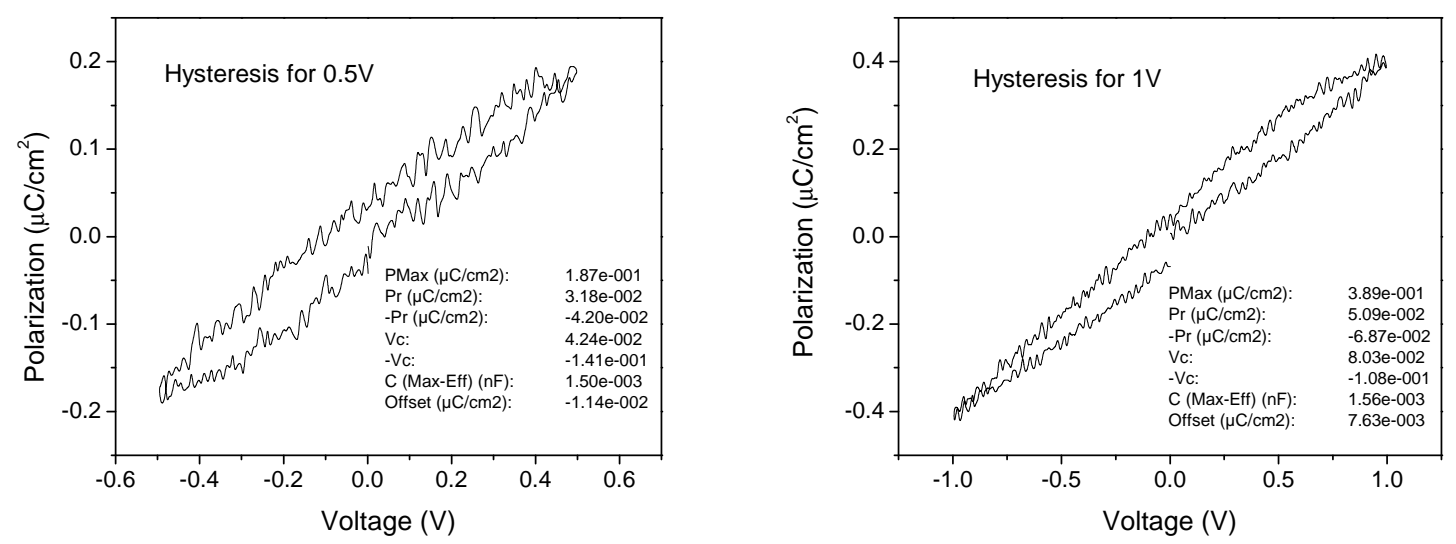

Figure 114: $P-V$ loops for $0.5 \mathrm{~V}$ and $1 \mathrm{~V}\left(875^{\circ} \mathrm{C}\right)$.

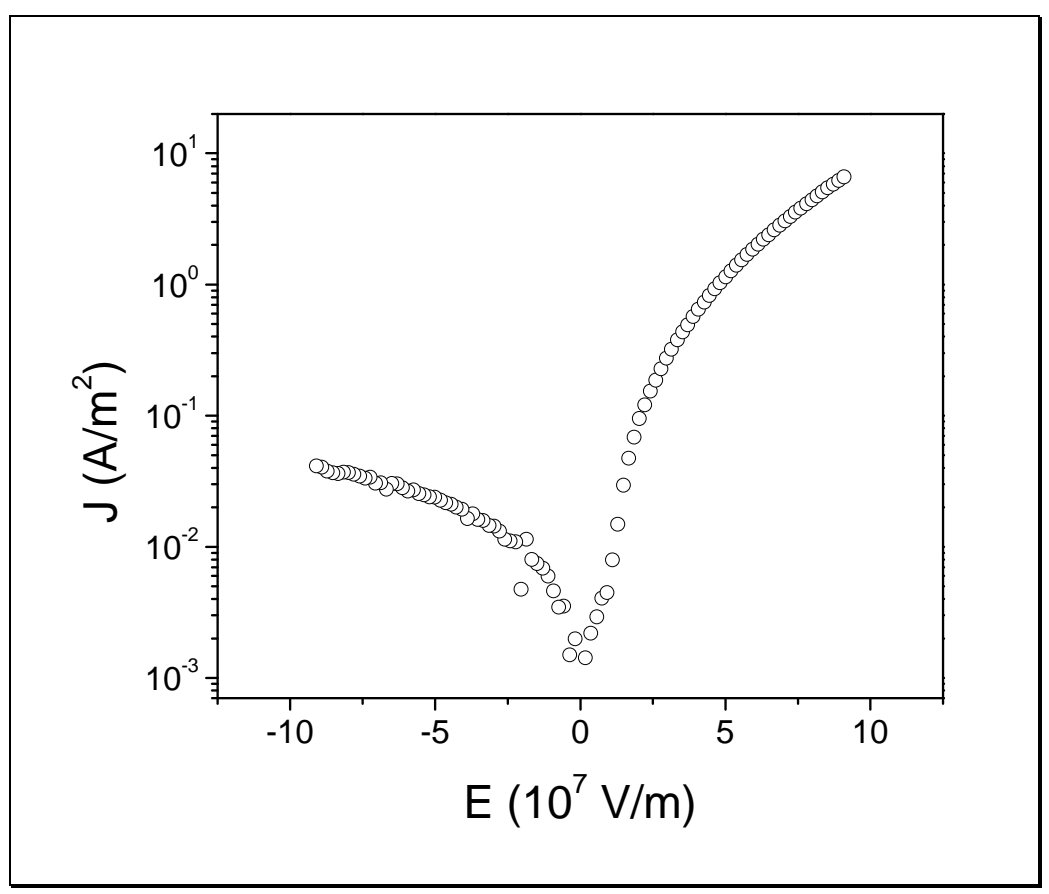

Figure 115: $J$ vs. $E$ for $875^{\circ} \mathrm{C}$. 


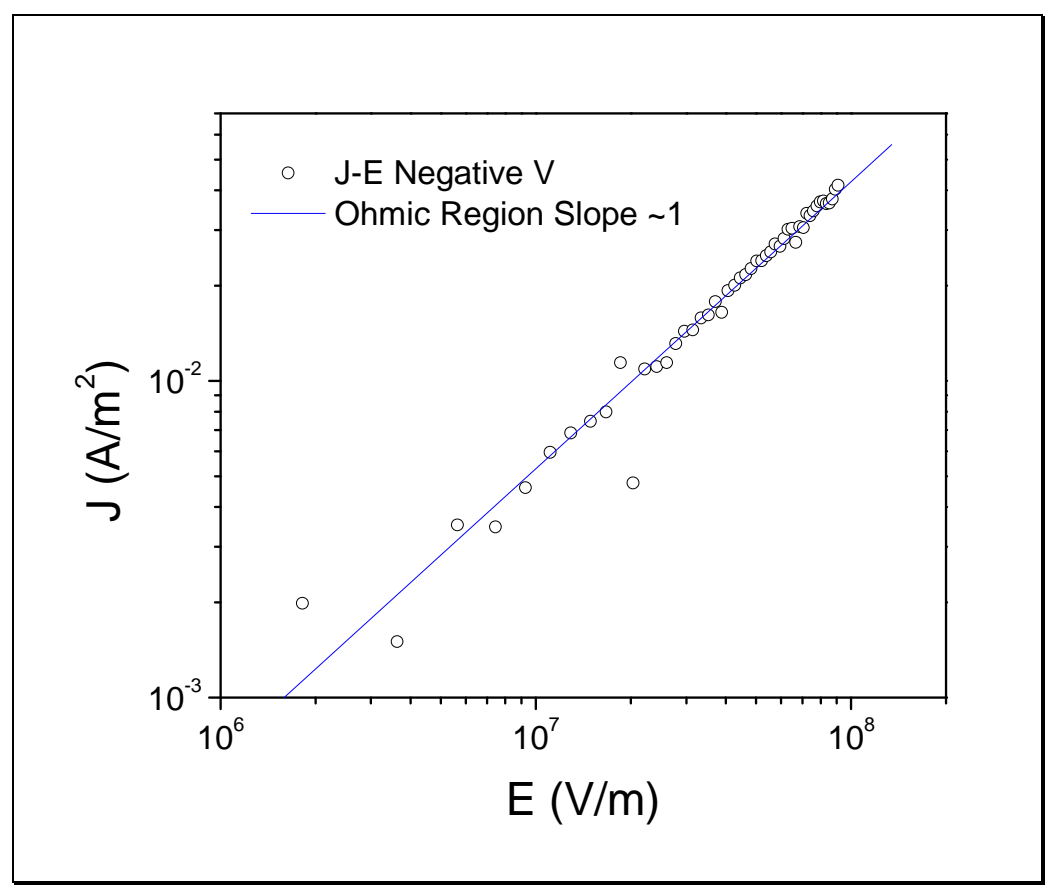

Figure 116: $\log J$ vs. $\log E$ for negative voltage $\left(875^{\circ} \mathrm{C}\right)$. Slope is $\sim 1$ across entire range.

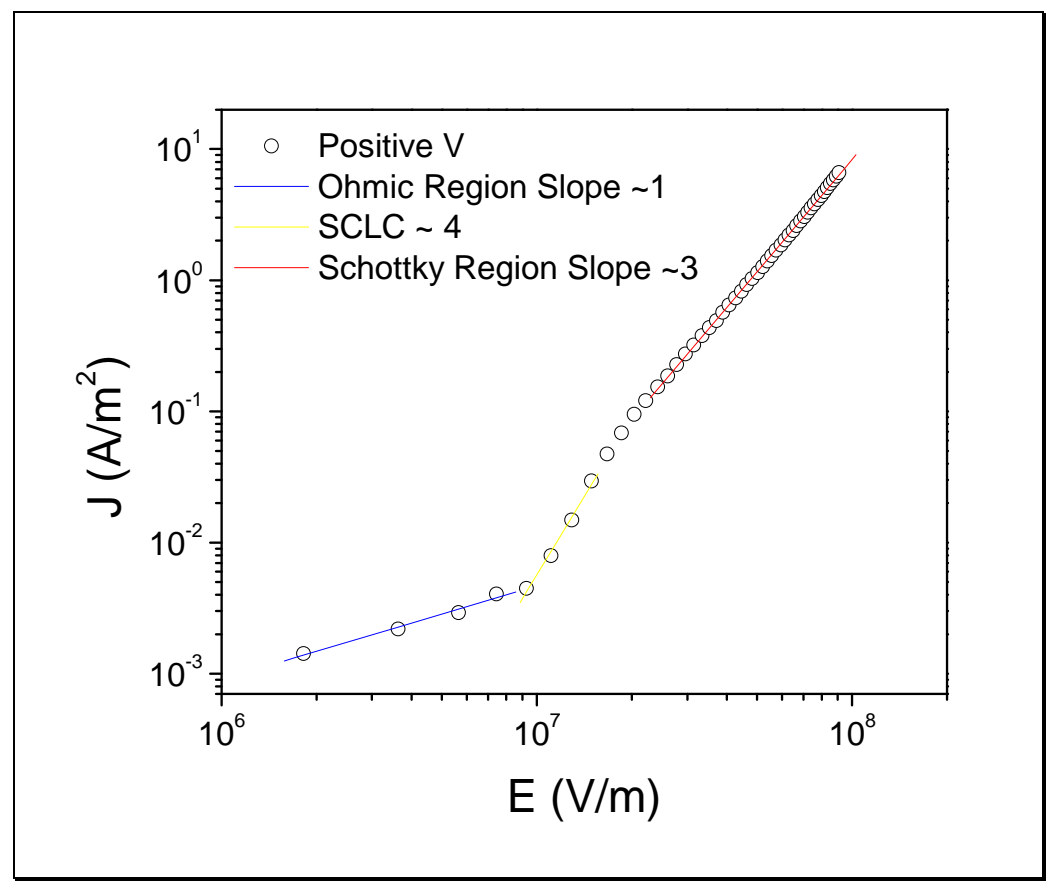

Figure 117: $\log J$ vs. $\log E$ for negative voltage $\left(875^{\circ} \mathrm{C}\right)$. Each solid, colored line represents a linear fit to a separate leakage region. 


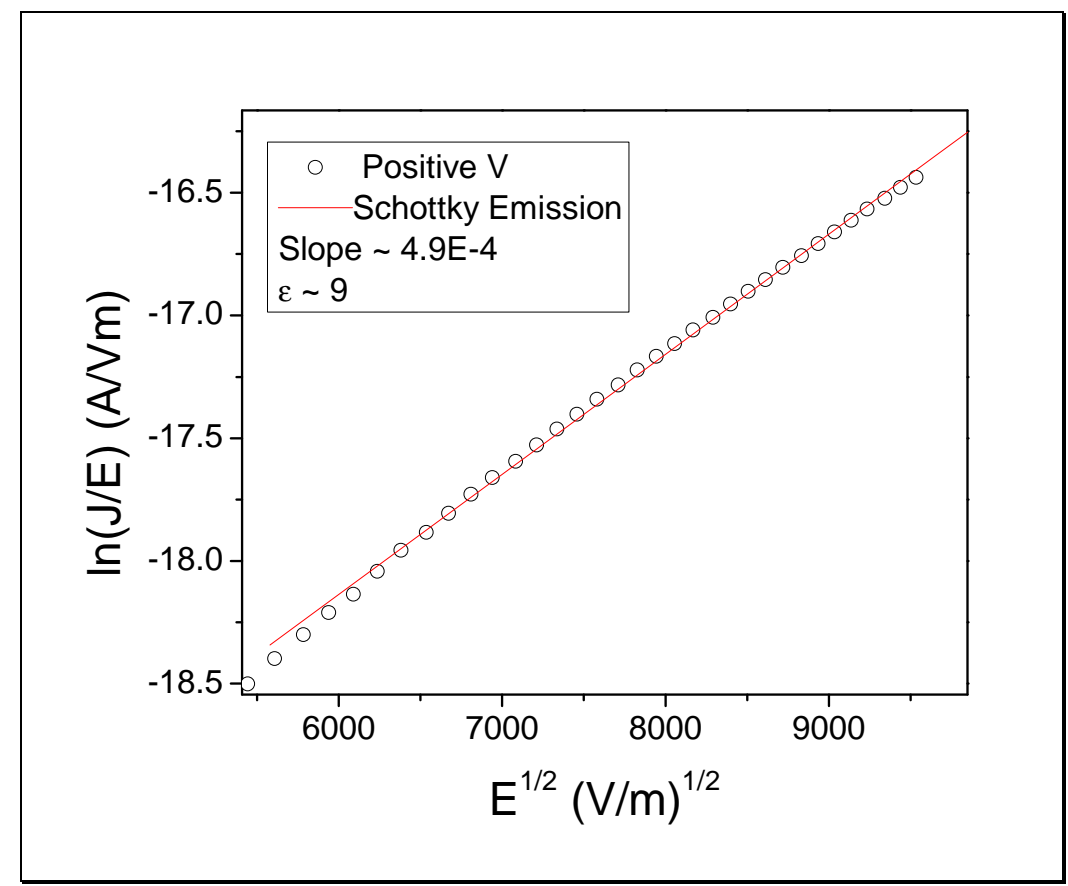

Figure 118: $\operatorname{Ln}(J / E)$ vs. $\mathrm{E}^{1 / 2}$ for negative voltage $\left(875^{\circ} \mathrm{C}\right)$. The line is the linear fit to region corresponding to Schottky emission.

\section{A.8.5 $\quad T_{\text {sub }}=900 \mathrm{C}$}

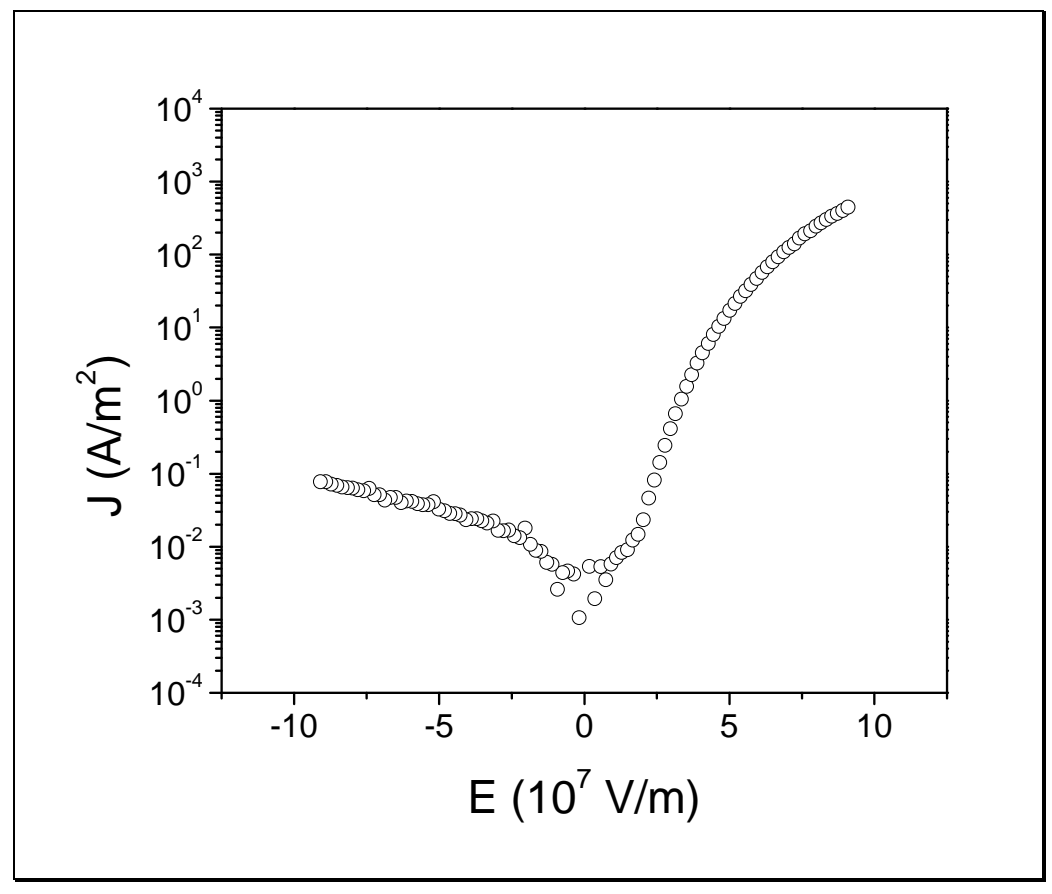

Figure 119: $J$ vs. $E$ for $900{ }^{\circ} \mathrm{C}$. 


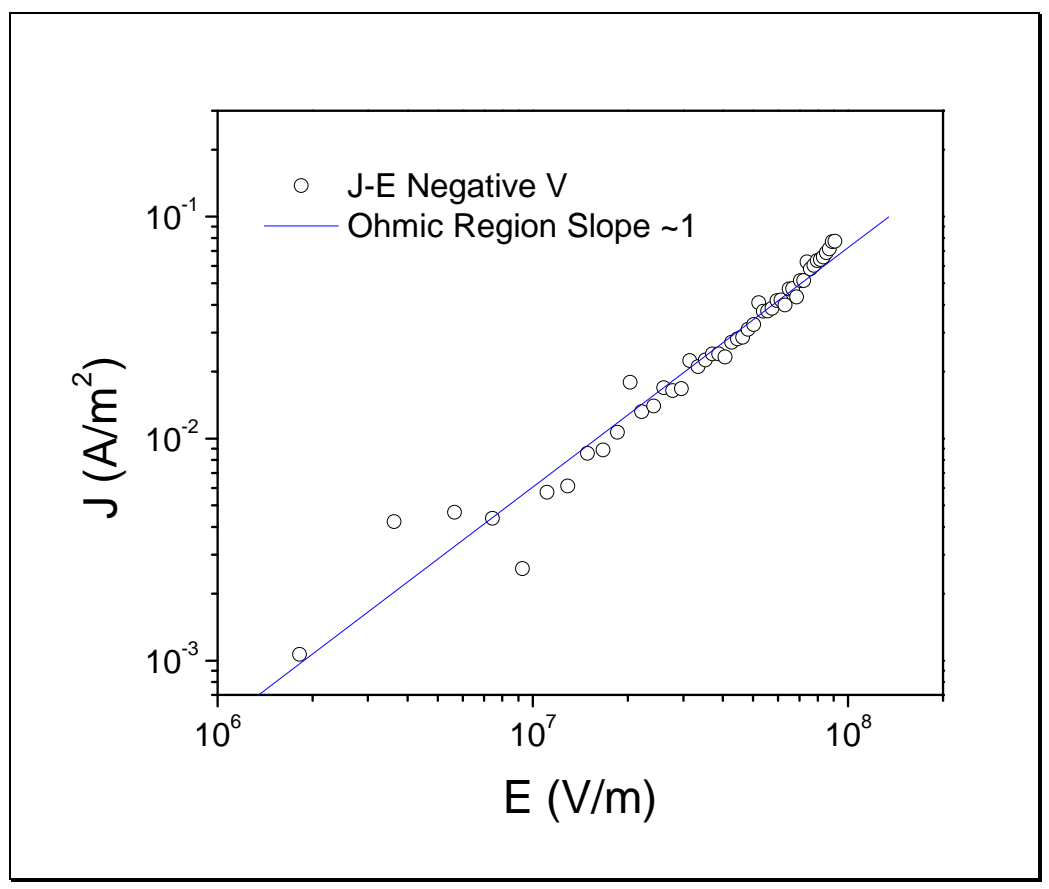

Figure 120: $\log J$ vs. $\log E$ for negative voltage $\left(900{ }^{\circ} \mathrm{C}\right)$. Slope is $\sim 1$ across entire range. 